\title{
IntechOpen
}

\section{Basic Pharmacokinetic Concepts and Some Clinical Applications}

Edited by Tarek A Ahmed 



\section{BASIC \\ PHARMACOKINETIC \\ CONCEPTS AND SOME \\ CLINICAL APPLICATIONS}

Edited by Tarek A Ahmed 
Basic Pharmacokinetic Concepts and Some Clinical Applications

http://dx.doi.org/10.5772/58502

Edited by Tarek A Ahmed

\section{Contributors}

Lawrence Lee, Kok-Yong Seng, Qigui Li, Suriyaprakash Tnk, Sakthivel Lakshmana Prabu, Sureshkumar Raman, Suggula Sairatan, Yakkaala Anilraju, Mayuki Aibiki, Noriyasu Fukuoka, José M. Lanao, Tarek A. Ahmed

\section{(c) The Editor(s) and the Author(s) 2015}

The moral rights of the and the author(s) have been asserted.

All rights to the book as a whole are reserved by INTECH. The book as a whole (compilation) cannot be reproduced, distributed or used for commercial or non-commercial purposes without INTECH's written permission.

Enquiries concerning the use of the book should be directed to INTECH rights and permissions department (permissions@intechopen.com).

Violations are liable to prosecution under the governing Copyright Law.

\section{(c)) BY}

Individual chapters of this publication are distributed under the terms of the Creative Commons Attribution 3.0 Unported License which permits commercial use, distribution and reproduction of the individual chapters, provided the original author(s) and source publication are appropriately acknowledged. If so indicated, certain images may not be included under the Creative Commons license. In such cases users will need to obtain permission from the license holder to reproduce the material. More details and guidelines concerning content reuse and adaptation can be foundat http://www.intechopen.com/copyright-policy.html.

\section{Notice}

Statements and opinions expressed in the chapters are these of the individual contributors and not necessarily those of the editors or publisher. No responsibility is accepted for the accuracy of information contained in the published chapters. The publisher assumes no responsibility for any damage or injury to persons or property arising out of the use of any materials, instructions, methods or ideas contained in the book.

First published in Croatia, 2015 by INTECH d.o.o.

eBook (PDF) Published by IN TECH d.o.o.

Place and year of publication of eBook (PDF): Rijeka, 2019.

IntechOpen is the global imprint of IN TECH d.o.o.

Printed in Croatia

Legal deposit, Croatia: National and University Library in Zagreb

Additional hard and PDF copies can be obtained from orders@intechopen.com

Basic Pharmacokinetic Concepts and Some Clinical Applications

Edited by Tarek A Ahmed

p. $\mathrm{cm}$.

ISBN 978-953-51-2197-8

eBook (PDF) ISBN 978-953-51-7265-9 


\section{We are IntechOpen, \\ the world's leading publisher of Open Access books}

Built by scientists, for scientists

\section{$3,800+$}

Open access books available

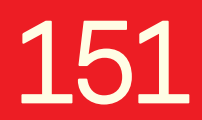

Countries delivered to

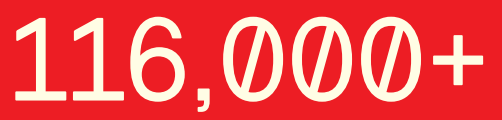

International authors and editors
$120 \mathrm{M}+$

Downloads

Our authors are among the

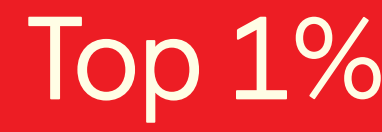

most cited scientists

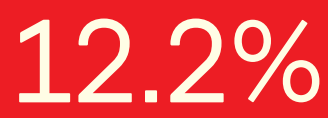

Contributors from top 500 universities

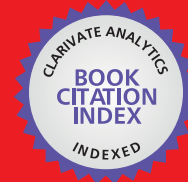

WEB OF SCIENCE ${ }^{\mathrm{TM}}$

Selection of our books indexed in the Book Citation Index in Web of Science ${ }^{\mathrm{TM}}$ Core Collection (BKCI)

Interested in publishing with us?

Contact book.department@intechopen.com

Numbers displayed above are based on latest data collected.

For more information visit www.intechopen.com

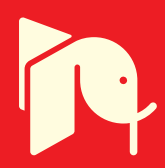





\section{Meet the editor}

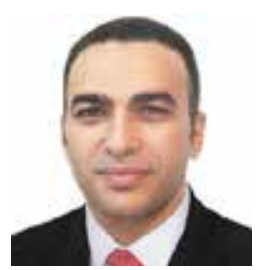

Dr. Tarek A Ahmed completed his undergraduate and master's degree from the Faculty of Pharmacy, Al-Azhar University, Cairo, Egypt. He received his $\mathrm{PhD}$ from Texas A\&M Health Science Center, Texas, USA. Over the years, Dr. Tarek gained vast experience and excellent teaching skills through his handling of all of the pharmaceutical practical and theoretical courses for undergraduate and postgraduate students, especially those that deal with pharmacokinetics and biopharmaceutics. Besides his teaching skill, Dr. Tarek has a lot of international publications in different pharmaceutical scientific research journals such as International Journal of Pharmaceutics, Journal of Microencapsulation, AAPS PharmSciTech, Drug Development and Industrial Pharmacy, Pharmaceutical Development and Technology, International Journal of Drug Delivery, International Journal of Nanomedicine, Life Science, and others. 



\section{Contents}

Preface XI

Chapter 1 The Impact of Pharmacokinetic Mismatched Antimalarial Drug Combinations on the Emergence and Spread of Drug Resistant Parasites 1

Qigui Li and Mark Hickman

Chapter 2 Pharmacokinetic (PK) Characteristics in Critically III Patients 33

Mayuki Aibiki and Noriyasu Fukuoka

Chapter 3 Pharmacokinetics of Drugs Following IV Bolus, IV Infusion, and Oral Administration $\mathbf{5 3}$

Tarek A. Ahmed

Chapter 4 Drug Distribution and Drug Elimination 99

Seng Kok-Yong and Lee Lawrence

Chapter 5 Biopharmaceutics and Pharmacokinetics 117

S. Lakshmana Prabu, T.N.K. Suriyaprakash, K. Ruckmani and R.

Thirumurugan

Chapter 6 Clinical Pharmacokinetic Applications of

Recirculatory Models 137

José M. Lanao and Clara Isabel Colino 



\section{Preface}

The purpose of this book is to provide undergraduate and postgraduate students of pharmacology with the basic as well as the newly applied concepts of pharmacokinetics. An overview of the concept of pharmacokinetics and biopharmaceutics in addition to the scientific terms used in pharmacokinetic equations along with some examples and basic problems in pharmacokinetics are illustrated. This will help undergraduate students understand the concept of absorption, distribution, metabolism, and elimination from common routes in the body following drug administration. The book also highlights clinical aspects in the pharmacokinetics of some antimalarial drugs and spread of drug resistance, recirculatory models.

Each chapter in this book is written at a level that is appropriate for students or those who are interested in this area of study and contains an introduction that clarifies the basic concepts described therein.

Finally, we acknowledge the effort and contribution of all the authors, coauthors, editorial members, and others who helped in bringing this book to light.

Tarek A Ahmed, Ph.D.

Associate Professor of Pharmaceutics

Department of Pharmaceutics

Faculty of Pharmacy, KAU 

Chapter 1

\title{
The Impact of Pharmacokinetic Mismatched Antimalarial Drug Combinations on the Emergence and Spread of Drug Resistant Parasites
}

\author{
Qigui Li and Mark Hickman \\ Additional information is available at the end of the chapter
}

http://dx.doi.org/10.5772/59506

\section{Introduction}

The concept of drug combination therapy is defined on the premise of combining drugs with similar half-lives in order to keep a constant dual pressure on current and reinvading parasites, which provides improved therapeutic efficacy and delays the development of resistance to the individual partner drug [1]. As the science of pharmaceutical technology and chemical synthetic capability advances is developed, the antimalarial drug combination has become more sophisticated and data driven, rather than empirically derived. The most significant problem with antimalarials is the growing incidence of drug resistance to $P$. falciparum. Drug resistance implies that there is a right shift in the relationship between drug concentration and efficacy. Although treatment failure in malaria usually results from poor patient drug compliance, inadequate dosing, pharmacokinetic (PK), and pharmacodynamic (PD) factors, some malaria infections will recrudesce and none of these factors will adequately explain why a recrudescence occurred. How parasites persist despite apparently adequate antimalarial treatment is an unanswered question [2,3].

Combination therapy (CT) with antimalarial drugs is the simultaneous use of two or more blood schizonticidal drugs with independent modes of action and different biochemical targets in the parasite. Artemisinin based combination therapy (ACT) has been adopted in Asia since 1992 and in Africa since 2000 and ACTs have shown improved treatment efficacy with reductions in drug toxicity. ACTs are now the treatment of choice for $P$. falciparum malaria globally. They are more effective than non-artemisinin combinations or monotherapies, and their use reduces the probability of drug resistance emerging in $P$. falciparum parasites. Most of the antimalarial drugs and their combination formulations now in use were introduced before the modern era of dosage design based on PK principles to minimize 
drug resistance [4]. Antimalarial dose regimens have tended to be empirically derived based on clinical studies with drug half-life, PK inter- individual variation, and drug exposure variables derived from experimental data or referred to by data from public trials. PK studies involve investigating the relationship between absorption, distribution, metabolism, and excretion of a drug. Many drugs share the same metabolic pathways or target the same receptors when considering matching their metabolism for selection of antimalarial combination drug partners. The ideal PK properties for an antimalarial drug have long been debated. From a resistance prevention perspective, the combination partners should have similar PK properties (PK half-life matching) [5].

Current recommended ACTs have repeatedly shown in a variety of clinical studies that the ACT partner drugs are well matched, efficacious well tolerated, and provide protection against new infections. ACTs are founded on the very novel mechanism of action of the artemisinin partner, which provides rapid parasite killing of the parasite biomass, quick reduction in clinical symptoms, broad spectrum killing against drug resistant parasites, and downstream decreases in formation of gametocytes, which in turn diminishes the spread of drug resistance genes [6]. An ideal drug combination would consist of drugs possessing complementary halflives to provide growth inhibition from two drugs on existing and new infections. Drug possessing a short half-life are preferred to limit drug exposure at lower, non-growth inhibitory concentrations to new parasitic infections. By administering drugs with short half-lives, the exposure of drug to parasites is limited and the pressure to select for drug tolerance and resistance is minimized. However, as recently discussed, administration of PK mismatched antimalarial drug combinations does not significantly impact the spread of resistance, and administration of PK mismatched ACTs have a known risk of jeopardizing the efficacy of partner drugs where resistance has not yet been widely observed [7, 8]. Although PK matching has been required by the FDA for new anti-infective drug combinations, simply matching halflives has not been considered sufficient [9].

PK mismatched drug combinations such as the ACTs have not been shown to have a significant risk of increasing drug resistance. Thus, the hypothesis that treatment with PK mismatched ACTs leads to drug resistance would seem to be rejected. In addition, clinical data shows that drug regimen that have significant PK mismatches have more mismatched regimens have consistently been associated with better and faster parasite killing than perfectly matched regimens. With that being said, there are examples of antimalarial drug combination that precede the ACTs with mismatched drug half-lives that have rapidly failed due to drug resistance. One of the best examples is the combination of mefloquine and sulfadoxinepyrimethamine (Fansi-Mef). Mefloquine has a longer half-life than either sulfadoxine or pyrimethamine and drug resistance problems were predicted when this combination was introduced; this prediction was shown to be accurate.

Two aspects are important when choosing drug combination partners designed with the aim of containing the development and spread of drug resistance: mechanisms of action that are synergistic but different and a good PK match. Antimalarial drug combination treatment based on artemisinin drugs is recommended for first-line treatment worldwide due to high efficacy and a demonstrated ability to deter drug resistance. By considering factors beyond matching 
half lives one could reasonably consider other influences such as drug mechanisms, other PK parameters beyond half-life, $\mathrm{PD}$, and malaria epidemiology, and consideration of all of these factors should result in creation of more effective combination regimens that retain therapeutic and prophylactic efficacy in the face of drug resistance. No doubt, PK and PD matched drug combinations could enhance treatment efficacy and delay drug resistance, however, the pharmacological mismatch hypothesis as applied to antimalarial drugs is countered by clinical data.

The use of drug half-lives is an overly simplistic approach to explain why drug resistance has developed given the requirement to have active inhibitory concentrations of both partner drugs present at the same time to deter resistance to either drug partner. It is entirely possible to have partner drug concentrations trailing off below the minimum inhibitory concentration at a time when the calculated drug half-life suggests antiparasitic protection. Consequently, it appears more important that drug antimalarial activity profiles post-treatment are the critical factors to be matched, rather than simply their half-lives. The PD profile after dosing is dependent on dosage administered, the half-lives of the drugs administered, the PD parameters of the drugs administered, and any drug-drug interactions that may occur [1]. Many factors that might influence efficacy and resistance of antimalarial combinations for both drug treatment and chemoprophylaxis indications include convenience of the prescribed antimalarial regimen in PD matching. The PD matching parameter of drug killing activity can be quantified by assessing the drug time above the minimal inhibitory concentration (MIC) in plasma. Time above MIC for any antimalarial drug is a function of the maximum concentration $\left(\mathrm{C}_{\max }\right)$ and drug clearance, which are again dependent on the dose administered and the formulation. The interaction between factors such as half-life, dosage, partner drug, and parasite drug sensitivity will yield hopefully a well-designed CT. In addition, those factors allow for mismatch in any of these variables by altering the relative dosages to achieve matched activity profiles post-treatment [1]. There are a variety of considerations to achieve a partner drug PD match to include different modes of action, different drug interactions of synergism and antagonism, avoidance of toxicity for any one partner drug, and CT or ACT-driven parasite killing.

While the PK mismatch hypothesis has been refuted for emergence of long-lived drug resistance, this hypothesis may be relevant for some geographic areas with endemic malaria [9]. In areas of high malaria transmission, the use of artemisinin derivatives might have little or no effect on malaria incidence. The use of drug combination treatment, however, may have an effect on the emergence of drug resistance in these endemic locations. While artemisinin compounds do not provide protection for longer-lived partner drugs given their rapid clearance, artemisinin drugs can diminish the probability of selection of drug resistance parasites initially and through enhanced antimalarial efficacy, artemisinin drugs can reduce transmission of drug resistant parasites and decrease gametocyte carriage. One of the most likely reasons why ACTs have deterred the emergence and spread of drug resistance may be related to the pharmacodynamic effects of artemisinin, particularly the reduction in parasite burden of 10,000 fold during each asexual replication cycle. Most importantly, the combination antimalarial drug partner will provide reciprocal protection to the artemisinin derivative from 
drug resistance and toxicity. Rational consideration of PK/PD matching of drug partners should result in more effective combination regimens that retain therapeutic and prophylactic efficacy in the face of efficacy and resistance. Our view is that adopting a rational and objective method to simulate ACT or other CT drug effectiveness using PK/PD match and mismatch principles can play a valuable role in this process.

\section{Current ACT partner drugs selected by comparative clinical trials}

In the absence of an effective malaria vaccine, early and successful chemotherapy for malaria performs an essential role in reducing morbidity andmortality. Multidrug resistance has been reported from most parts of the world, and drug monotherapy or the use of some of the available combination chemotherapies for malaria is either ineffective or less effective. New antimalarial regimens are urgently needed and ACTs are widely advocated as the best antimalarial regimens possible today. ACTs have been shown to increase efficacy, shorten duration of treatment (and hence increase compliance), and they have been shown to decrease the risk of resistant parasites arising through mutation during therapy. There are five ACTs used broadly for first-line treatment of $P$. falciparum malaria globally, and these five artemisinin combination treatments have been shown to be much more effective than non-artemisininbased combinations or monotherapies. The ACT options now recommended by WHO [2] for treatment of uncomplicated falciparum malaria include:

- artemether plus lumefantrine (AL),

- artesunate plus amodiaquine (AS-AQ),

- artesunate plus mefloquine (AS-MQ),

- artesunate plus sulfadoxine-pyrimethamine (AS-SP), and

- dihydroartemisinin plus piperaquine (DP).

Despite these advances, better treatments are required, and this leads us to the associated requirement to insure comparative efficacy assessments are well designed and easily interpreted. Given the performance of artemisinin combination therapy, the minimum acceptable cure rate has been at least $90 \%$. This expectation is far above the cure rates achieved with malaria monotherapy in past years where cure rates of $70 \%$ were considered state of the art [2, 3]. The other side of this expectation is the idea that if cure rates fall below $90 \%$ a change in drug is therefore required. The overall effect of ACT use results in reduction in the probability of parasite recrudescence, reductions in the within-patient selection pressure, and prevention of parasite transmission. If the cure rate of any ACT is below $90 \%$, the recommendation for the use of that combination for treatment should change, and a better ACT should be selected based on data from a comparative efficacy study demonstrating clinical superiority. In earlier "superiority trials", it was reasonable to plan a randomized comparison to test if there was a difference between the regimens being tested. 
There is a wealth of published data on malaria drug studies with ACTs for treatment. One problem readily observed when comparing these studies is trying to compare data from studies with differing study endpoints. The WHO has updated its recommended study endpoints incorporating measures of both parasitological and clinical outcomes to standardize study design [3]. The majority of malaria drug studies conducted utilize clinical outcomes at either 14 or 28 days as the principal endpoint. This set of endpoints is too short, however, to account for the lingering presence of parasites still present as the presence or absence of residual parasites is an important public health parameter. Residual parasites still present in the blood of infected patients after treatment can result in anemia, recrudescent infection, and development of severe malaria. Longer-term studies conducted with an extended follow-up period can provide a more meaningful comparison of drug treatment in areas of high malaria transmission.

All ACTs in current clinical use are well designed and the studies supporting their use have been well conducted in various randomized comparative trials before being placed on market. Based on clinical experience with each partner drug in monotherapies and cautious consideration with various PK and PD factors for the combinations, the main comparative trials are summarized below along with discussion of non-ACT combinations previously in use.

\subsection{Non-ACT combinations are no longer recommended for the treatment of malaria}

There are a number of combination treatments that have been developed over the years that are not based on artemisinin drugs. These combinations include sulfadoxine-pyrimethamine plus chloroquine (SP-CQ) and sulfadoxine-pyrimethamine plus amodiaquine (SP-AQ). Given the broad distribution of resistance to antifolate compounds and to chloroquine, SP drug combinations are no longer effective. The combination of amodiaquine plus SP is more effective than either drug by itself, however, the efficacy of the amodiaquine sulfadoxinepyrimethamine combination is usually inferior to ACTs, and this combination is no longer recommended for the treatment of malaria. Only Malarone (atovaquone-proguanil) is still recommended for chemoprophylaxis use [2].

\subsection{ACTs recommended by the World Health Organization (WHO)}

\subsubsection{The 6-dose regimen of artemether-lumefantrine $(A L)$ is superior to a 4-dose regimen}

The data derived from one clinical trial conducted in Thailand from 1996-1997 (238 adults and children) showed a significantly higher rate of cure at day 28 with the 6-dose regimen of AL given over 3 days compared to the 4-dose regimen of AL also given over 3 days. To provide definitive data on this question of 4 doses versus 6 dose regimens, two six dose regimens comprised of a total of $480 \mathrm{mg}$ of artemether and 2,880 $\mathrm{mg}$ of lumefantrine were assessed against a 4 dose regimen comprised of $320 \mathrm{mg}$ of artemether and 1,920 $\mathrm{mg}$ of lumefantrine. This study was a double-blind trial 359 patients infected with uncomplicated multidrug resistant falciparum malaria. The study participants showed no differences in the fever and parasite clearance times, and there were no adverse effects noted with one regimen not observed in another. The six-dose regimens provided day 28 cure rates of $96.9 \%$ and 
99.12\%while the four dose regimen showed a cure rate of $83.3 \%(\mathrm{P}<0.001)$. Both of the sixdose regimens of AL were shown to provide a highly effective and very well tolerated treatment when compared to four-dose regimens of AL [10, 11].

\subsubsection{A six-dose regimen of $A L$ is not superior to treatment with $A S-M Q$}

In another set of clinical trials (537 participants), the efficacy of a six-dose regimen of AL was compared to treatment with AS-MQ. For the AL regimen, two studies compared the antimalarial efficacy of AL to treatment with AS-MQ with an endpoint of day 28 parasitemia, and no differences in parasite or fever clearance time were detected. There were 11 parasitological failures in the AL treatment arm and none with AS-MQ [12]. The data from this study suggests that AS-MQ therapy is more effective than AL treatment [13].

\subsubsection{The six-dose regimen of AL is similar to the efficacy achieved with AS-AQ treatment.}

To test the hypothesis that AS-AQ is as effective as AL for treatment of acute uncomplicated malaria in Nigerian children, an open label, randomized controlled clinical trial was conducted in children aged 6 months to 10 years. The 132 patients in this trial were split into two groups of 66 and received $4 \mathrm{mg} / \mathrm{kg}$ of AS plus $10 \mathrm{mg} / \mathrm{kg}$ of amodiaquine daily or a weight based administration of a fixed dose AL tablet administered twice daily. Treatment with AS-AQ and AL was conducted for three days and clinical follow up was conducted until day 28 . The cure rate in the protocol population at Day 28 for AS-AQ was 93\%, and the cure rate for AL was $95 \%$ (OR $-0.71,95 \%$ CI 0.12-3.99, rho=0.66). The median survival time for the AL treatment group was 21 days and the means survival time for the AL treatment group was 28 days (Kaplan Meier product limit estimates, $\mathrm{p}=0.294$ ). The polymerase chain reaction (PCR) corrected day 28 cure rates per protocol of the two patient populations were $98.4 \%$ for AS-AQ and $100 \%$ for AL. Both drug combinations were well tolerated, the efficacy comparison demonstrated that AS-AQ was as effective as AL, and both combinations were shown to be both efficacious and safe [14].

Another clinical trial was conducted in Burundi to compare children treated with AS-AQ versus AL with a 14-day follow-up period. A total of 295 children under 5years were included; 153 children were treated with AS-AQ, and 142 children were treated with the AL drug combination. Among the 295 children, 290 were followed up to 14 days. In the group of 149 children treated with AS and amodiaquine, 142 or $95.3 \%$ (95\% CI: 91.9-98.7\%) presented with adequate clinical and parasitological response, five or 3.3\% presented with late parasitological failure, one or $0.7 \%$ with a late clinical failure and one or $0.7 \%$ with an early treatment failure. Among the 141 children treated with AL, 140 or $99.3 \%$ (95\% Cl: 97.9-100\%) presented with adequate clinical and parasitological response and one or $0.7 \%$ presented with a late parasitological failure. Side effects were comparable in both groups except for vomiting. Vomiting was more frequent in the AS-AQ treatment arm on Day 1 and Day 2 while the AL arm did not show this side effect. Both treatments decreased gametocyte carriage but did not achieve full clearance in all patients. During a consensus workshop, the Ministry of Public Health agreed that the combination of AS-AQ would be the best choice for first line treatment of uncomplicated falciparum malaria in Burundi including epidemic outbreaks [15]. 


\subsubsection{The efficacy of DP is superior to $A S-A Q$ for treatment of uncomplicated malaria}

A comparative efficacy study was conducted to compare treatment with dihydroartemisininpiperaquine (DP) to treatment with AS-AQ. This trial compared the efficacy of DP versus AS AQ in 334 patients infected with $P$. falciparum, P. vivax or both species of Plasmodium (185 were infected with $P$. falciparum, 80 were infected with $P$. vivax, and 69 were infected with both species) with a 42-day follow-up period. The overall parasitological failure rate at day 42 was $45 \%$ for AS-AQ and 13\% for DP. Rates of both recrudescence of $P$. falciparum infection and recurrence of $P$. vivax infection were significantly higher after receipt of AS-AQ than after receipt of DP. By the end of the study, AS-AQ recipients were 2.95-fold more likely to be anemic and had a probability of carrying P. vivax gametocytes that was 14.5-times higher. DP demonstrated better efficacy and tolerability compared to AS-AQ for treatment of drugresistant falciparum and vivax malaria infections. The extended post-treatment prophylactic effect provided by piperaquine delayed falciparum re-infection, diminished the incidence of vivax infection, decreased clinical anemia, and decreased the potential for vivax gametocyte carriage [16, 17].

\subsubsection{The efficacy of DP is superior to a six-dose regimen of $A L$}

Based on clinical trial data, Uganda introduced AL as the treatment of choice for uncomplicated malaria treatment. While AL is well tolerated and efficacious, it does have some drawbacks as a drug combination to include a requirement for administration with fatty foods, a twice-daily dosing regimen, and an increased risk of malaria re-infection in endemic areas. One of the new alternatives to AL for treatment is the combination of dihydroartemisinin and piperaquine (DP). In contrast to AL, DP is dosed once daily, not twice, and due to the long elimination halflife of piperaquine, this drug combination has a long period of prophylaxis post treatment.

A comparison of DP to AL was conducted in Kanungu, which is known to have moderate transmission of malaria. The 408 patients infected with uncomplicated falciparum malaria completed a 42-day follow-up, and the patients ranged in age from 6 months to 10 years. To differentiate recrudescent infections from new infections, the parasites were genotyped. DPtreated patients showed a much lower probability of parasitemia recurrence as only $12.2 \%$ developed recurrent parasitemia while $33.2 \%$ of AL-treated patients developed recurrent parasitemia (risk difference of $20.9 \%, 95 \% \mathrm{CI}=13.0-28.8 \%$ ). There was no statistically significant difference between the two drug regimens due to recrudescent infections where $\mathrm{AL}$ treated patients showed recrudescence of 5.8\% while DP treated patients showed recrudescence of $2.0 \%$ (risk difference of $3.8 \%, 95 \% \mathrm{CI}=0.2-7.8 \%$ ). DP treated patients showed a diminished risk of gametocyte carriage after treatment as $4.2 \%$ showed gametocytemia after DP treatment while $10.6 \%$ showed gametocytemia after AL treatment $(p=0.01)$. Both AL and DP were shown to be safe and well tolerated $[18,19]$. Similar studies were performed by other scientists in different regions and these studies indicated that daily treatment with DP is more efficacious than treatment with the six doses AL regimen. DP was also shown to be superior to AL for reducing the risk of recurrent parasitemia and gametocytemia, and it was shown to provide improved hemoglobin recovery [18-20]. 


\subsubsection{The efficacy of DP is similar to AS-MQ}

Multi-drug resistant falciparum malaria is a significant health problem in the Peruvian Amazon region. A randomized open label clinical trial was carried out comparing AS-MQ, the current first line treatment in this region of Peru, with DP. Total 522 patients with uncomplicated falciparum malaria were enrolled in this study and 260 were treated with AS-MQ and 260 with DP and followed for 63 days. Clinical and parasitological responses adjusted by PCR and estimated using per protocol analyses and Kaplan Meier survival tests were very good for both AS-MQ and DP. AS-MQ showed a $99.6 \%$ response while DP showed a $98.4 \%$ response $(R R=0.99,95 \% C I=0.97-1.01)$. All of the incidents of recrudescence that were observed were attributed to parasitological failures that occurred late in the treatment period. All of the gametocytes observed were cleared in 28 days in the AS-MQ treatment group while gametocyte clearance in the DP treatment group required 35 days. New gametocytes were observed to appear more rapidly in patients treated with DP than in patients treated with AS-MQ. By day 7 eight patients treated with DP presented with gametocytemia while only two presented with gametocytemia in the AS-MQ group. Side effects of anxiety and insomnia were observed more frequently in patients treated with AS-MQ compared to DP. In summary DP was shown to be as efficacious as AS-MQ for treatment of falciparum malaria, and the DP combination is cheaper and better tolerated [21]. Other comparative efficacy studies have been conducted yielding similar data $[22,23]$.

\subsubsection{Other clinical trials}

A meta-analysis of data from 16 randomized trials (12 from Africa) compared the effect of adding 3 days of any artemisinin to one of the standard treatment regimens of $C Q, S P, A Q$, or $\mathrm{MQ}$. The conclusion drawn in this study from all of the clinical trial data examined is the addition of 3 days of artemisinin to any of the standard antimalarial treatments significantly reduced parasitological failure on days 14 and 28. Gametocyte carriage was also reduced in the artemisinin treated patients.

In further studies, 3-day treatments with $\mathrm{AL}, \mathrm{AS}-\mathrm{AQ}$, and DP was used for comparison of candidate regimens using 2-3 day courses of artemisinin-piperaquine (A-P). While initial parasite clearance was rapid and the A-P treatment was well tolerated, the 28-day cure rates for A-P were $<80 \%$ for 2-day treatments $(2.4 \mathrm{mg} / \mathrm{kg}$ artemisinin, $14.4 \mathrm{mg} / \mathrm{kg}$ piperaquine) and 3 day treatment with artemisinin-piperaquine $(3.2 \mathrm{mg} / \mathrm{kg}$ artemisinin and $16.0 \mathrm{mg} / \mathrm{kg}$ piperaquine) showed $>98 \%$ cure rates. These data supports further evaluation of 3-day treatment regimens with A-P for multidrug-resistant falciparum malaria. Similarly, data on the comparative efficacy of the chlorproguanil-dapsone -AS (CDA) combination are not yet available as clinical Phase III trials are still pending [17].

In conclusion, one non-ACT (atovaquone-proguanil) and five ACT combinations are recommended by the WHO to treat effectively drug resistant strains of Plasmodium [2]. In Southeast Asia, documented cases of resistance to artemisinins have been reported [24-26]. This resistance is characterized by delays in the parasite clearance time. Artemisinin resistance is related to widespread use particularly the indiscriminate use of artemisinin drugs as monotherapy. Due to the short half-lives of artemisinin compounds, cases of parasite reinfection have occurred during their use in monotherapy to treat malaria. The practice of prescribing 
artemisinin drugs as monotherapy also enhances the probability of selecting for artemisinin resistant strains of Plasmodium. Artemisinins need, therefore, to be associated with effective antimalarials, which have a relatively long half-life. Not all combinations are good alternatives. Clinical trials comparing the efficacy of the AS-SP combination have shown problems with SP efficacy as monotherapy, and the AS-SP combination should not be administered in areas where cure rates for SP monotherapy are less than 80\% [2]. AS-MQ and AS-AQ are both efficacious regimens, however, problems with tolerability with both drug combinations have been observed. The fixed-dose combination of AL remains very effective and well tolerated. It is recommended, however, that this combination be administered twice daily for three days with a fat-rich meal, which may limit adherence to treatment. In this context, the combination of DP shows equal or superior efficacy than other ACTs, and this combination appears to be a good alternative, particularly in malaria-endemic areas.

\begin{tabular}{|c|c|c|c|}
\hline Antimalarials & $\begin{array}{l}\text { Half-life of } \\
\text { artemisinin } \\
\text { derivative }\end{array}$ & $\begin{array}{l}\text { Half-life of long-term } \\
\text { partner drug } \\
\text { per full adult } \\
\text { course (US\$) }\end{array}$ & $\begin{array}{l}\text { Regions currently in } \\
\text { use purchase cost } \\
\text { per course (US\$) }\end{array}$ \\
\hline $\begin{array}{l}\text { Artemether-lumefantrine } \\
\text { (AL) }\end{array}$ & $\sim 3 h$ & $4-5$ days & $\begin{array}{c}\text { Africa, EM, SE Asia, WP } \\
\text { and SA }\end{array}$ \\
\hline $\begin{array}{l}\text { Artesunate-mefloquine } \\
\text { (AS-MQ) }\end{array}$ & $<1 \mathrm{~h}$ & 14-21 days & $\begin{array}{c}\text { Africa, SE Asia, WP and } \\
\text { SA }\end{array}$ \\
\hline $\begin{array}{l}\text { Artesunate-amodiaquine } \\
\text { (AS-AQ) }\end{array}$ & $<1 \mathrm{~h}$ & 9-18 days $\ddagger$ & Africa and EM \\
\hline Chloroquine $^{1}$ & - & $1-2$ months & $\begin{array}{c}\text { Africa, EM, SE Asia, WP } \\
\text { and SA }\end{array}$ \\
\hline Dihydroartemisinin-piperaquine (DP) & $45 \mathrm{~min}$ & $\sim 5$ weeks & SE Asia \\
\hline $\begin{array}{l}\text { Artesunate-SP } \\
\text { (AS-SP) }\end{array}$ & $<1 \mathrm{~h}$ & 4 days $(S)$ or & $\begin{array}{l}\text { Africa, EM (IPT in } \\
\text { Africa, EM and WP) }\end{array}$ \\
\hline Atovaquone-proguanil (Malarone) & NA & $\begin{array}{l}\sim 8 \text { days }(\mathrm{P}) \\
73 \mathrm{~h} \text { (Ato) or } \\
\text { 14h (Prog) }\end{array}$ & $\begin{array}{c}\text { Africa, SE Asia, EM and } \\
\text { WP }\end{array}$ \\
\hline
\end{tabular}

*This refers to the $t_{1 / 2}$ of the active metabolite monodesethyl-amodiaquine; the $t_{1 / 2}$ of amodiaquine is $\sim 3 h r$.

${ }^{1}$ These former first-line antimalarials are included as a reference. EM, eastern Mediterranean; IPT, intermittent preventive treatment; NA, not applicable; $\mathrm{P}$, pyrimethamine; $\mathrm{S}$, sulphadoxine; SA, South America; SE Asia, Southeast Asia; $\mathrm{t}_{1 / 2}$, halflife; WP, Western Pacific. SP = sulfadoxine-pyrimethamine

Table 1. Plasma half-lives of drugs used in artemisinin-based combination therapies [28]

None of the comparative efficacy trial designs for these combinations followed the PK match hypothesis that the drugs contained in a regimen should have elimination half times that match and that the partner drugs should not have any clinically significant negative PK 
interactions [27]. The elimination half-lives of partner drugs should match because the matching elimination kinetics is especially important in areas of high malaria transmission due to exposure of parasites to declining concentrations of one drug in the combination leading to development of resistance. All six artemisinin drug combinations show a high degree of PK mismatch (Table 1).

\section{Matching drug half-lives to deter drug resistance}

Resistance to antimalarials by falciparum parasites has been an ongoing global public health concern since chloroquine and mefloquine resistance emerged [28]. The emergence of multidrug resistant parasites has triggered the use of combination antimalarial regimens. The need for effective treatments has resulted in use of antimalarial combinations thrown together that have often worked better than monotherapies, though sometimes only temporarily. Many factors that might influence efficacy and resistance of antimalarial combinations for both chemotherapy and chemoprophylaxis indications include convenience of the prescribed antimalarial drugs rather than a focus on matching PK parameters. Therefore, the ideal drug combination would consist of drugs with complementary half-lives to insure each drug provides inhibitory activity to protect the other $\operatorname{drug}(\mathrm{s})$ in a combination from emerging drug resistance associated with exposure to sub-therapeutic monotherapy. To avoid such exposure to sub-therapeutic drug levels, drugs with shorter half-lives would be a better choice to avoid exposure of new parasites to sub-therapeutic drug levels. Once the artemisinin component has been eliminated, the partner drug is therefore left unprotected once the artemisinin has been eliminated from the body, and selective pressures on that partner drug will lead to development of resistance.

The implications of this "PK mismatch", particularly in areas of high transmission in Africa, requires more investigation and trade-offs between prevention of resistance and protection of patients from recrudescence and development of new infections may be considered. The safest approach from a perspective of preventing development of drug resistance is to use a drug partner that has a residual half-life as short as possible, while still enabling parasite clearance with a 3-day treatment. This ideal PK/PD matched combination may be difficult to develop given the limited range of antimalarial drugs available. When combinations are used, mismatched PK profiles can play a role in facilitating development of resistance. Mismatched PK profiles allow parasites to evolve resistance sequentially as the longer half-life partner persists as a vulnerable monotherapeutic agent (Figure 1). The results of mismatched PK profiles can almost completely undermine the benefits of combination therapy.

Coartem (artemether-lumefantrine) is one of the first combination treatments with artemisinin drug to be formulated as a fixed dose to enhance patient compliance. Data on drug resistance profiles [29] suggests this combination may be vulnerable to certain mutations in the pfmdr1 multi-drug resistance transporter gene found in P. falciparum. Mutations in the pfmdr1 gene from $Y$ to $\mathrm{N}$ (position 86) have been shown to lead to increased tolerance to Coartem facilitating parasite re-infection. While the $86 \mathrm{~N}$ mutation appears to be acting to enhance drug tolerance 


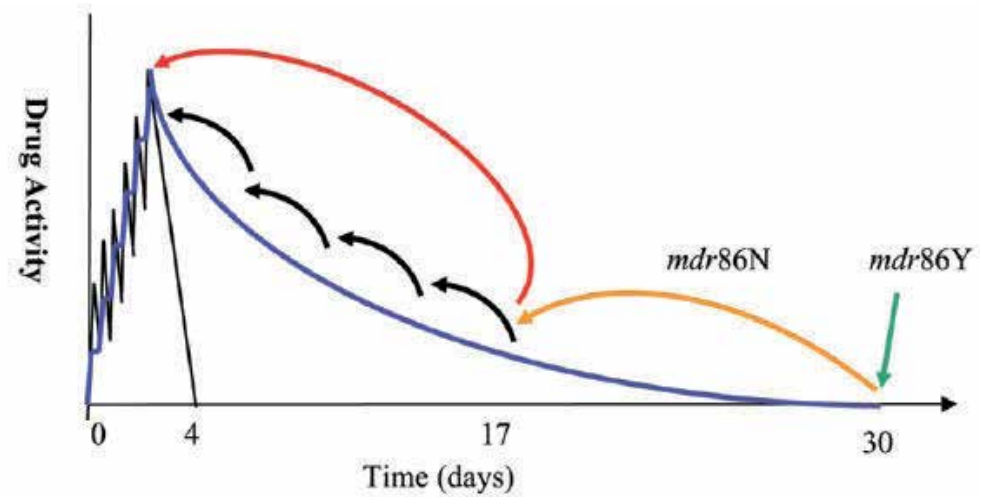

Figure 1. Likely evolution of resistance to artemether-lumefantrine $(\mathrm{AL})$, the graph represents the drug levels in the serum of a patient starting the 3-day Coartem therapy regimen, an artemisinin-based combination therapy (ACT) comprising lumefantrine (blue) and artemether (black). The drugs have decayed to non-therapeutic levels when they cross the $X$-axis (defined as the concentration at which the drug, if present as a monotherapy, would be unable to prevent infection by drug-sensitive parasites). The $p f m d r 86 \mathrm{~N}$ mutation's (orange arrow) increased tolerance of lumefantrine allows it to infect a person 17 days post-treatment, compared with 30 days post-treatment for the mdr86Y mutation. Two scenarios are given for subsequent mutational steps. Scenario 1 is a single large increase in drug tolerance (red arrow), in which the presence of artemether in the ACT is powerless to stop the spread of this mutation. The increased tolerance of lumefantrine allows it to infect a person 4 days post-treatment and to spread through the parasite population, displacing the $86 \mathrm{~N}$ form, which can infect no earlier than 17 days post-treatment. Scenario 2 is a subsequent increase in tolerance, which occurs as a series of small increments (black arrows); the presence of artemether in the ACT will slow this process when it reaches day 4 , and Coartem may remain clinically effective for a longer period [29].

rather than act as a mechanism of drug resistance, parasites with this mutation will spread this drug resistance allele more readily than wild-type parasites with the $86 \mathrm{Y} \mathrm{pfmdrl}$ gene. (Figure 1).These observations raise a new additional point: can ACT use accelerate the development of resistance to artemisinin derivatives? The presence of the $p f m d r l ~ 86 \mathrm{~N}$ allele has also been shown to confer less sensitivity to artemisinin in vitro [29].

While artemisinin or an artemisinin derivative may not have selected for resistance at pfmdr1 $86 \mathrm{~N}$ due to the short half-life of artemisinin drugs, lumefantrine has a much longer half-live of 5 days could be driving selection at this allele resulting in increases in drug tolerance to both partners. This study does support the principle that understanding mechanisms of resistance at the molecular level to ACT partner drugs is essential to maintain drug efficacy of these combination treatments. ACTs, like other drugs, have a finite useful life span, which likely varies from short in Southeast Asia to longer in Africa. Development of new ACT drugs with better-matched PK rates of clearance is clearly a priority to avoid emergence of drug resistance to both drugs in the combination. To observe resistance emergence with clarity, early detection is clearly essential to facilitate longer term planning on first-line drug choices [30].

Mathematical modeling of associations between drug clearance and parasite drug resistance have been proposed based on a division of parasite resistance into three categories, Res0, Res1, and Res2. Parasites in the Res0 group have wild-type susceptibility to antimalarial drugs, and they are readily killed. Parasites in the Res1 group have increased tolerance to antimalarial drugs, but they can still be killed. Parasites in the Res2 group are resistant to drug treatment 
and cannot be killed. The rate of parasite evolution in the Res2 group is commonly underestimated if the effects of drug clearance are not taken into account. There are also two phases of drug resistant development related to the speed of resistance development, which are intrinsic to this model of drug resistance. In the first phase, Phase A, Res1 drug tolerant parasites are spreading and replacing Res0 drug sensitive parasites, however, Res2 parasites are not present. In Phase B, parasites capable of surviving drug treatment are more quickly selected which leads to emergence of Res2 parasites, which cannot be killed, and clinically, results in treatment failure. Clinical treatment of malaria parasites in Phase A is commonly successful, and the transition from Phase A to Phase B is not readily observable without drug resistance testing of parasite populations. This underscores the need for parasite drug surveillance programs to sample parasite populations and assess any transition from wild type to drug resistant [31].

The addition of this aspect of PK to model of drug resistance has resulted in several novel findings, as detailed earlier. 1) A long elimination half-life at therapeutic concentrations results in long periods of chemoprophylaxis, so drug half-life is a potent selective force increasing the rate at which resistance evolves. 2) Parasite drug resistance arising from mutations at a particular gene locus may take place in two phases, Phase A and B, where drug tolerance evolves into overt drug resistance. 3) The duration of Phase A and Phase B may be quite variable depending on the transmission rates in a particular geographic area, which underscores the need for active drug surveillance programs. 4) Artemisinin drug combination therapy has been shown to slow down the rate of parasite drug resistance, however, successful introduction of an ACT is dependent on the absence of drug resistance to either of the partners in the ACT as existing resistance to either partner will result in rapid clinical failure [31-33].

All currently used combinations of artemisinins have mismatched drug elimination rates, and the pharmacokinetic half-life differences between the short-lived artemisinins and the longlived quinolines such as piperaquine and mefloquine is a cause for concern regarding the longterm development and spread of drug resistance [8]. The elimination half-life of artemisinins ranges from 0.4-2.6 hours, but the elimination half-life of quinolines range from 4 days-2 months. The elimination half-life for sulfadoxine-pyrimethamine (SP) ranges from 4-8 days, and the elimination half-life of mefloquine (MQ) ranges from 14-21 days. Accordingly, there is an extended period when SP and MQ are "unprotected" given the short half-life of AS (Table 1) [32-34]. This sort of mismatched PK profile occurs with other combinations such as AL, AS$\mathrm{AQ}, \mathrm{DP}$, and the combination of atovaquone-proguanil used in Asia and Africa [8, 35]. Artemisinin derivatives are protected from the rapid development of resistance as these drugs circulates in the presence of a much more long-lived partner, but the long-lived partner is not protected by the artemisinins [36].

Combining artemisinins with quinoline compounds has provided a mixed outcome. While the mechanisms of action of artemisinin and quinoline compounds provide a synergistic outcome, the elimination half-life differences between these compound classes is a risk for development of drug resistance. One example of this concern, which has proved to be on target, is the rise of AS-MQ clinical treatment failures in Southeast Asia in areas with high levels of MQ resistance. The combination of AS and MQ represents a drug combination with a significant mismatch in elimination half-lives $(<1$ hour for AS and 14-21 days for MQ). From a PK 
perspective, artemether and lumefantrine have a better match in terms of half-life as lumefantrine has a half-life of 4-5 days [37]. Despite this better match in half-lives, AL is slightly less efficacious than AS-MQ in areas with a high prevalence of multidrug resistant parasites $[38,39]$. AS-AQ has been recently introduced in regions of Sub-Saharan Africa. AS-AQ also suffers from a PK mismatch due to the long half-life of amodiaquine, which ranges from 9-18 days [40]. A number of studies in Africa have shown increases in drug failures associated with resistance to amodiaquine [41]. Drug resistance is likely to increase and spread given AS-AQ has been adopted as the treatment of choice in a number of countries in Africa [8].

The introduction of mefloquine into Southeast Asia resulted in development of drug resistance in a period of 4 years. This lead to efforts to create a combination drug comprised of mefloquine and sulfadoxine pyrimethamine (MQ-SP or FansiMef). This drug combination ultimately failed, and this failure was predicted in advance due to the mismatch in drug half-lives between mefloquine and sulfadoxine pyrimethamine. Mefloquine has a half-life of 14-21 days while pyrimethamine, the longer-lived of the two antifolate drugs, has a half-life of 10 days. This mismatch left mefloquine unprotected from development of parasite drug resistance during a period of sub-therapeutic treatment in the bottom half of the elimination period [42]. In contrast, the introduction of artesunate combined with mefloquine lead to significant reductions in malaria deaths, reductions in drug resistant parasites, and documented reversals in mefloquine drug resistance [43]. If SP could not have been expected to protect MQ against tile emergence of resistance because of a PK mismatch, why did the AS-MQ drug combination with an even more profound half-life mismatch (AS $<1$ hour, MQ 14-21 days) succeed [5]?

Whether the combination treatment failures are principally related to PK mismatch or not, the solution to the mismatch problem is conceptually straightforward: formulate and administer combination therapies in which pharmacokinetic profiles superimpose. PK match is not the only relevant factor and other factors, which influence treatment failure, such as poor patient compliance with therapy regimens, combination drug interaction, PD profiles, and drug related toxicity, should still be considered.

\section{PK mismatch does not lead to emergence of ACT resistance}

\subsection{Simply matching PK half-lives is not sufficient to design the best drug combinations}

Artemisinin derivatives are particularly effective when used as combination therapies because they are well tolerated, have excellent parasiticidal properties, but there is currently early evidence of clinical drug resistance [24-26]. PK matching has been required by regulatory agencies such as the FDA for anti-infective drug combinations; however, simply matching the half-lives of prospective ACT partner drugs will not be adequate to provide dual pressure on falciparum parasites [5]. One example of this is the chloroquine which has a very long half-life of 4-6 weeks, however, drug elimination is not constant and drug concentration above the minimal inhibitory concentration is likely much shorter. This leads to exposure of parasites to sub-therapeutic drug concentrations. The time when antimalarial drugs persist at subtherapeutic concentrations is defined as the "selective window". During this selective window 
period, the concentration of antimalarial drugs is sufficient to inhibit the growth of drug sensitive parasites but insufficient to prevent the growth of drug resistant parasites. As chloroquine has a long period of sub-therapeutic drug concentration (and hence a long selective window) this explains why chloroquine is vulnerable to emergence of drug resistant parasites.

When exposed to two drugs, parasites would need to develop mutations at two different genes simultaneously, and the likelihood of this occurring (i.e., $10^{-6} \times 10^{-6}=10^{-12}$ ) becomes extremely small [44]. The probability of selecting a mutant parasite with resistance to both drugs is very unlikely. Quick parasite killing by artemisinin compounds results in very few parasites left to become drug resistant, however, artemisinin monotherapy must be administered for 7 days which does not work well in practice given poor adherence to this regimen, and 7 day regimens of artemisinin compounds have been shown to fail in 10\% of subjects [45]. ACT combinations must include a drug partner which has a half-life greater than 24 hours. By combining an artemisinin drug with a slowly eliminated compound such as MQ (half-life of 20 days) given for 3 dayscomplete protection is provided for the artemisinin derivatives ( $<1$ hour half-life) from drug resistance. $M Q$, however, is left unprotected, which demonstrates the mismatch in drug partner PK profiles. The number of residual parasites exposed to MQ alone, following two asexual cycles, is a tiny fraction of those present at the peak of the acute symptomatic infection. In such regimens, therefore, the PK profile mismatch is unavoidable.

The residual parasites left after artesunate killing are subsequently exposed to elevated concentrations of mefloquine. Despite any presence of drug tolerant parasites, the concentrations of mefloquine present are likely sufficient to kill the remaining residual parasites. However, the long elimination half-life of mefloquine will provide a filter to select for the survival of drug resistant parasites newly acquired which will enhance the spread of drug resistant parasites. In low transmission areas of northwestern Thailand where mefloquine resistant parasites are present, the use of artesunate-mefloquine was very efficacious in treatment of drug resistant parasites and inhibiting malaria incidence [43, 46]. AS-MQ will likely be useful in other regions such as in Africa where higher transmission rates of malaria are present.

There are major concerns that mismatched PK profiles will allow parasites to evolve resistance to the longer half-life partner, undermining the benefits of ACTs. Drug combinations with mismatched PK profiles do not decrease the spread of resistance and drug partners should have complementary PK profiles to insure drug pressure from both partners is sufficient to avoid selecting for drug resistance. Artemisinin 'resistance' or 'tolerance' has developed in Southeast Asia manifested by delayed parasite clearance and reduced sensitivity to ACT [3]. In vivo data derived from PK/PD studies should provide the basis for defining the time above the minimum inhibitory concentration required for treatment and further characterize the selective windows that partner drugs provide. Both critical pieces of PK/PD data will help drive partner selection of drug combination. Each new combination derived will present with advantages and disadvantages based on cost, tolerability, PK profile matching, and ease of administration. 
Drug combination therapy has been experimentally assessed for treatment of multidrug resistant tuberculosis, which in a variety of ways, mimics some of the challenges faced today for antimalarial drug development. Just as in antimalarial drugs, the hypothesis that PK-profile mismatch could explain the emergence of drug resistance was tested using two common antituberculosis drugs, rifampin, and isoniazid. The hypothesis being tested was the larger the PK profile mismatch, the greater the size of isoniazid and rifampin resistant bacterial subpopulations would become. To test this hypothesis, investigators examined the sterilizing and bactericidal effects of hollow-fiber-system studies in experiments lasting up to 42 days. These experiments were designed to mimic the PK of rifampin and isoniazid administered to patients. Rifampin was administered first, followed by isoniazid 0, 6, 12, and 24 hours later.

Statistical analysis of the different combination regimens showed that the 12 hour and 24 hour mismatched regimens consistently killed better than the PK matched regimens for both sterilizing effects and bactericidal effects $(p<0.05)$. The results from this experiment suggest changing order of administration or scheduling of drug administration could result in enhanced microbial killing. A number of analysis of variance calculations were conducted in this study, and the authors concluded that rifampin-resistant and isoniazid-resistant subpopulations were not significantly increased due to PK mismatch. Thus, the PK mismatch hypothesis was rejected to explain development of drug resistant tuberculosis. Instead, the authors concluded that sequential administration of anti-tuberculosis drugs following a particular schedule is the best new paradigm for accelerating killing of M. tuberculosis. In addition, the authors concluded that current efforts aimed at better PK matching to decrease tuberculosis resistance emergence are likely futile and counterproductive [9].

\subsection{PK mismatch does not lead to emergence of resistance to ACTs}

Antimalarial drugs of the quinolines, antifolates, and artemisinin classes have been deployed in various combination therapies with each other to both increase the efficacy of treatment as well as delay the incidence of drug resistance. In most of the ACTs currently in use or being evaluated, e.g. AS-MQ, the partner drug is eliminated slowly. The partner drug is therefore unprotected once artemisinin has been eliminated from the body, and this lack of protection for the long-lived partner creates selective pressure for emergence of new drug-resistant infections. The consequences of using drugs with mismatched PK profiles are not understood particularly in regions of high malaria transmission found in Africa. The safest approach is to combine drugs with the shortest elimination half-life capable of clearing parasites in a 3-day drug treatment regimen. Given the array of drugs on hand, this is not easy to do.

All of the antimalarial partner drugs on market have half-lives ranging from 5 days to 30 days, which leaves these partner drugs open to the eventual development of drug resistance [7]. Conversely, the use of long-lived partner drugs does provide a very good post-treatment prophylactic effect, which reduces the probability of future new infections. This extended post treatment prophylactic effect may come at the expense of the longevity of the drug combination treatment as drug resistance arising from PK profile mismatch could limit the time a particular combination therapy is effective. Drug combinations designed to deter emergence of drug resistance should have two important features; different, synergistic mechanisms of action and 
a compatible PK profile. As reviewed by Hastings and Watkins [7], drug combinations with PK profile mismatches do not deter the spread of drug resistant parasites and could cause problems with the efficacy of currently used still effective drugs. This underscores the need for compatible PK profiles among combination drug partners ideally with the shortest halflife possible [8].

In another study, the in vitro susceptibility of parasites to MQ was followed in patients treated with the AS-MQ drug combination. Although the combination of AS and MQ constitutes a PK mismatch, there has been no decline in the in vitro susceptibility of parasites to MQ during a 5-year study period; in fact, the sensitivity to MQ has increased significantly. This contrasts with a $40 \%$ decrease in efficacy in vivo to MQ in the 5 years before the study [47]. Cure rates with AS-MQ remain almost $100 \%$. The protective effect of AS on MQ when both drugs are administered in a combination regimen is believed to be against resistant parasites, which ensures high cure rates. In addition, the considerable reduction of parasite biomass by artesunate, which reduces the chance of selecting MQ-resistant mutant-parasites, and artesunate driven reduction in parasite transmission through reduction of gametocyte carriage rates are additional benefits provided by this combination $[48,49]$. These factors reduce the selection of MQ-resistant parasites [50]. This study has shown that through a 5-year study period that artesunate-mefloquine remained effective with no decrease in malaria drug sensitivity observed. This data may have been superseded by recent reports of artesunate drug resistance associated with a new molecular marker, mutations in the propeller region of a kelch protein, K-13 [24-26, 46].

Dihydroartemisinin -piperaquine (DP) is an ACT with a very large PK profile mismatch as piperaquine is characterized by the longest mean terminal elimination half-life when compared to other long-lived partners (Table 1). The cumulative risk of any parasitological failure, however, was greater for the AL, AS-MQ, and AS-SP combinations than for DP, which reflects the post-treatment prophylactic effect piperaquine given its long biological half-life of piperaquine. Various formulations of DP have been shown to be highly effective for treatment of uncomplicated P. falciparum and vivax malaria. A number of trials have shown that DP is similar or superior to AL, AS-MQ, AS-AQ, and AS-SP for treatment of malaria in children and adults in Asia and Africa. Fortunately, DP does not have the same selective pressure, and likely not the same mechanisms of resistance as chloroquine and amodiaquine. Piperaquine offers many advantages as a combination partner for treatment of uncomplicated malaria including superior efficacy, lower toxicity, and long-duration prophylaxis. Accordingly, the DP combination offers significant benefits over other available ACTs making it a prime option for the management of uncomplicated malaria [16].

Another important finding which does impact on the pharmacological mismatch hypothesis as applied to antimalarial drugs is clinical data that shows that more mismatched regimens have consistently been associated with better and faster microbial killing than perfectly matched regimens. Pharmacokinetically, the combination of artemether and lumefantrine is better matched compared to other ACTs (Table 1). It appears to be slightly less efficacious, however, than AS-MQ in areas with a high prevalence of multidrug resistant parasites [39]. In one clinical trial (537 participants), the efficacy of a six-dose regimen of AL was compared to AS-MQ. No differences in day 28 parasitemia were noted, and no differences in parasite or fever clearance times were detected. There were, however, 11 parasitological failures with AL 
and none with ASMQ [12]. The results of this clinical trial suggest that AS-MQ therapy is more effective than AL therapy $[13,51]$. This also suggests that solutions that rely on minimizing PK mismatch, such as fixed dose combinations, and the design of regimens that rely on better PK profile matching to close the monotherapy "window," will likely be ineffective solutions for combating drug resistance.

\subsection{The Impact of PK Matching on Malaria Parasite Epidemiology}

Antimalarial drug resistance usually starts in low transmission areas. Patients infected with falciparum malaria from regions with low or intermediate malaria transmission are generally symptomatic and require full treatment with ACT drugs to achieve a cure. Accordingly, the treatment of these patients is more likely to select for drug resistant parasites. By contrast, patients infected with falciparum malaria from regions with higher rates of transmission have a greater innate immunity developed through sequential infections, which may or may not be fully treated in low transmission areas, and it is in such areas that the benefits of antimalarial combinations would be greatest [49].

Optimized treatment plans must consider malaria transmission intensity as the probability of a new infection occurring during the post-treatment drug elimination phase equates to a tradeoff in terms of the elimination half-life required for an ACT partner. Exposure of patients to new malaria infections in high transmission areas occurring every day or every week may translate to a need for longer post-treatment prophylaxis rather than complete parasite eradication. Longer half-life drugs have enhanced risk for driving parasite drug resistance. For patients who are exposed to intermittent infections in low transmission areas, complete parasite eradication to deter drug resistance may be the more important requirement and mismatched PK profiles between partner drugs may be of less consequence [17].

The rejection of the PK mismatch hypothesis for emergence of long-lived drug resistance may not be applicable, however, to all malaria areas. In areas of high malaria transmission, use of artemisinin derivatives might have little or no effect on malaria incidence. The elimination half lives of partner drugs should match because the matching elimination kinetics is especially important in areas of high malaria transmission due to exposure of parasites to declining concentrations of one drug in a drug combination, which selects for resistance. Semi-immune individuals commonly harbor sexual and asexual parasite forms, which, in turn, increase the number of gametocytes present, which may offset any gains in transmission reduction through ACT treatment in endemic areas [49].

\section{PK profile matching is one of many strategies to prevent or slow the spread of drug resistance}

All CTs and ACTs currently recommended by the WHO rely on partner drugs that are already compromised by resistance including the artemisinins [24-26]. As ACTs are rolled out, optimizing their effectiveness and slowing their resistance will require selection of partner drugs based on PK and PD parameters, and such trials will target the right drugs to the right 
settings, which consider the broader impacts of different treatment regimens. New WHO policies are made based on good clinical research, and the mission to replace failed malaria drugs with more efficacious ACTs is being accomplished. However, maximizing the benefit and prolonging the life of ACTs will also require learning from the past. New strategies are needed to prevent ACTs from established drug resistance existing against partner drugs. Better understanding of the drug mechanisms of action, the PK profile match, PD profiles, drug toxicity, and malaria epidemiology will be required to design and deploy well-suited combination therapies that provide prolonged prophylactic efficacy while still deterring resistance. To delay the onset of resistance, the drugs used in combination should have compatible PK and PD profiles, no adverse pharmacological interactions, and no additional toxicity.

Artemisinin resistance has been confined to Southeast Asia [24-26], however, there is no reason why artemisinin resistance could not break out in other regions. Clinical research has shown the combination of artemisinin with an efficacious partner drug can improve cure rates to an adequate level even in areas where partner drug resistance has been observed [25]. Previous models demonstrating the benefits of ACT in delaying the onset of resistance largely assumed parasites are either completely sensitive to the drug, or else completely resistant [52]. This is an overly simplistic approach to modeling drug resistance, and knowledge of PK/PD mechanisms can guide development of more realistic models of drug resistance [53]. While PK profiles describe the disposition of drugs by the human body, PD parameters actually measure how drugs act on the parasite. PD parameters that are relevant include fever clearance (FCT) and parasite clearance times (PCT), time above the minimum inhibitory concentration (MIC), the half maximal inhibitory concentration (IC50) or accumulation of drug inside the parasite working at the drug target. This can be described by defining PK parameters after treatment and combining that data with PD parameters in vivo. Unlike models based strictly on differences in in vitro drug sensitivity or resistance, these combined PK/PD models do throw light on what properties define a good $\mathrm{CT}$, and therefore, we believe that a combination of $\mathrm{PK} / \mathrm{PD}$ considerations can usefully contribute to the rational design of drug combinations.

Antimalarial drugs are primarily deployed as combination therapy as a mechanism to prevent or slow the spread of resistance. Hastings and Hodel have defined six key PK/PD considerations for potential combination therapies [1]:

\subsection{The half-lives and activity profiles of partner drugs}

Mutual protection between partner drugs is dependent on having active concentrations of both drugs at the same time during treatment, and post-treatment. It, therefore, appears more important that drug antimalarial activity profiles post-treatment are the critical factors to be matched, rather than simply their half-lives. One way in which matching of drug killing can be quantified is by their time above the minimal inhibitory concentration (MIC) a concept originally used in bacteriology but now being increasingly used in malaria.

The time above the MIC is determined by the pharmacokinetic profile of a drug, and a number of parameters such as the maximum concentration attained and drug clearance affects this parameter. The MIC itself has been approximated by many using in vitro data derived from drug potency testing where the $\mathrm{IC}_{99}$ is used to approximate the MIC. Determining the time 
above the MIC can be approximated graphically by plotting the MIC concentration against the drug concentration versus time curve. By considering the interaction between a variety of PK and PD factors, combination drugs can be designed to minimize the effect of an adverse parameter by altering dosing to achieve a better PK/PD match post-treatment. Maximal concentrations of both drugs optimize clinical effectiveness and help protect against resistance being driven through drug failures [31]. The natural variation in PK/PD may largely undermine matching done on average PK/PD values.

\subsection{Natural variation in population $P K / P D$}

The variation in drug sensitivity for a parasite is a key PD parameter assessed in vitro by drug potency testing with an IC50 endpoint (the half-maximal inhibitory concentration). By assessing, the average IC50 values against a battery of parasites from different regions, better PD matches can be made when considering the variation that naturally may occur particularly when drug potency is assessed against multi-drug resistant parasites. Understanding the variation in parasite drug sensitivity is an important component for creating a PD profile match [54]. Constructing a balance between drug half-lives, dosing, and sensitivity of parasites to antimalarial drugs must be conducted based on population averages. Use of average values does have its drawbacks, as there is considerable variability in both PK and PD parameters in a population. Mismatches in PK and PD parameters will no doubt occur which may affect individual treatment of patients and may affect the extent to which mutual drug protection can be provided by each partner drug. Most arguments for matching half-lives are overoptimistic in nature and fail to consider how factors such as natural variation in PK/PD can affect a match made solely on one PK parameter. In conclusion, it is only plausible to make approximate matches based on drug half-lives. Most drugs outside the artemisinin class have relatively long half-lives, and they can be approximately matched with the limitations listed above.

\subsection{Dosages and toxicity}

Combining two drugs always increases the risk of toxicity, but it is important to quantify this increased risk and to describe how it can be mitigated. There are two main types of toxicity associated with malaria drugs. One type is dose- or concentration -dependent adverse drug reactions (ADRs), which are mostly predictable and consistent between patients because they are explained by the known pharmacological action of the drug. The second type is dose or concentration independent, and this type of toxicity is largely unpredictable and dependent on the metabolism, immune system, or genetics of individual patients. Changes in dosing of a combination with decreased concentrations of each partner drug requires drug efficacy modeling using both PK and PD parameters to answer the questions associated with the outcomes of reducing the doses of partner drugs in a combination treatment which may have an impact on both efficacy and drug resistance [54]. 


\subsection{Mechanisms of action}

Independent mechanisms of action provide complementary pharmacodynamic effects, and, indeed, drug combinations may provide benefits of drug synergy against malaria parasites that go beyond simple additive effects (drug combinations may also have antagonistic effects which are not helpful for combinatorial efficacy). Combining drugs with additive or synergistic action should also increase parasite clearance post-treatment and hence may speed the resolution of symptoms. One good example of such a drug combination is Malarone ${ }^{\mathrm{TM}}$, the combination of atovaquone and proguanil. The addition of proguanil, a weak antifolate drug, provides significant synergy to the action of atovaquone against the malaria parasite. Atovaquone as a single agent is quite vulnerable to development of malaria drug resistance (one single base pair mutation in the malaria parasite's $c y t b$ gene equates to complete drug resistance to atovaquone). Another well-known example is the synergy observed between artesunate and mefloquine; the combination of the two drugs together provides greater efficacy than the action of each individual drug. Drug synergy can provide significant benefits to overall efficacy, but any observed synergy should not necessarily be used to decrease the doses of each partner drug in a combination except where necessary to diminish toxicity. The ideal approach is to dose each drug at a monotherapeutic dose in a combination to maintain efficacy of the combination even when drug resistance is present against one partner drug.

\subsection{PK Profiles}

Drugs in combination treatments may have different pharmacokinetic profiles when dosed together compared to when they are dosed individually. Process of drug elimination and clearance should include an examination of the effects on drug disposition of dosing two drugs at the same time. If drugs share the same conversion or elimination pathways post absorption, then their actions could become non-additive but in unpredictable ways. Conversely, if the same elimination pathway saturates for both drugs, and both parent forms are active, then drug half-lives may be extended and anti-malarial synergy may arise. In essence, the consequences of PK interactions are difficult to predict and may be dependent on exact metabolic pathways utilized for drug elimination, which determines whether active metabolites have antiparasitic activity. PK interactions can be readily assessed in both in vitro and animal models prior to administration to patients in clinical trials. Early drug safety and pharmacokinetic studies are generally conducted using healthy volunteers, and the pharmacokinetic effects of malaria infection cannot be assessed definitively in these early studies.

\subsection{Common mechanisms of resistance}

Combination therapy provides benefits to patient care based on the need for a parasite to develop resistance to both drugs to avoid being killed. Intuitively, cross-resistance between the constituent drugs in a CT will undermine this effect and modelling shows that even small amounts of cross-resistance may have an adverse effect on the usable lifespan of both drugs in a combination [55]. Combining drugs from the same class into a combination treatment is not the best practice $[2,4]$ given mutations may arise that provide resistance to both drugs in the combination. This has been shown to occur with antifolate drug combinations such as 
sulfadoxine-pyrimethamine. A more significant drawback to using drugs from the same class is the likelihood that both drugs actually share the same drug target and potentially a similar mechanism of drug toxicity.

PK/PD matching of partner drugs is mainly achieved through deployment of appropriately matched partner drugs in combination to enhance efficacy and reduce the probability of selecting for drug resistance. In many models of drug resistance, this is modeled as purely a parasite specific component that will inform whether a patient will potentially be cured by a malaria drug. A PK/PD model based on drug mechanism of action is structured differently and the potency of a drug against the malaria parasites (as assessed by the IC50) is one of many possible variables that may influence treatment outcome. In order to model why individual patients fail treatment, it is essential to know how a particular drug combination actually clears an existing infection. Putting resistance into this matrix of variables will inform the PK/PD parameters associated with treatment success and failure. Accordingly, rational and objective models based on PK/PD parameters are the best tools available to project whether a particular combination treatment is a good choice for a particular area [1].

Treatment of malaria disease today is focused on using combination therapies, and defining $\mathrm{PK} / \mathrm{PD}$ parameters accurately to achieve the best drug match which is essential to reduce the speed at which resistance develops. Therefore, three more PK/PD considerations for potential combination therapies are required which we will discuss in detail below.

\subsection{Partner drug-drug interaction}

Drug-drug interactions occur mainly during drug absorption and during the phase of drug distribution. These processes are dependent on active transport pathways, plasma protein binding of drugs, drug biotransformation, and excretion. Most drugs are given orally, and subsequently, drug absorption occurs through the intestinal mucosa, mainly in the duodenum. The bioavailability of drugs is dependent on a P-glycoprotein found in the enterocytes of the intestine.

The Pglycoprotein can actively pump back drugs back into the intestinal lumen, which in turn drops the bioavailability of any orally dosed compound. Metabolism of compounds is dependent on the activity of cytochrome P450 (CYP450) enzymes found in enterocytes, which can metabolize drugs before they reach systemic circulation. Induction or inhibition of the Pglycoprotein and enterocyte CYP450 enzymes can thus influence drug bioavailability. In patients infected with falciparum malaria, concomitant administration of oral artemisinin and mefloquine has been shown to significantly increase the AUC and decrease the oral clearance of artemisinin. In contrast, concomitant oral administration of dihydroartemisinin (DHA) and mefloquine to healthy volunteers has been shown to have no effect on either partner drug, except for a slight increase in the absorption rate of mefloquine. Similarly, no alteration in the pharmacokinetic s of either atovaquone or proguanil was observed following concomitant treatment with artesunate (AS) in healthy adult volunteers [56]. 


\subsection{Drug-tolerant parasites}

ACTs may also decrease transmission of drug-resistant parasites by reducing the number of gametocytes carried by patients. Optimal combination plans must consider the intensity of malaria transmission because the likelihood of a new infection after the drug elimination phase translates into a consideration between shorter-acting or longer acting combination drugs. Patients living in endemic areas who experience new infections on a regular basis may benefit more from a longer period of post-treatment prophylaxis than absolute eradication of all infecting parasites. This benefit may be worth using a longer duration partner drug that is more prone to develop drug resistance and hence have a shorter life span. Overall, the goal of deterring drug resistance must be achieved through elimination of parasite transmission. In 1996, Kyle et al [57] described the dormancy theory, in which some of the parasites, called "sleeping beauties" exposed at ring stage in vitro become metabolically inactive and resume growth after removal of the drug. Others have since confirmed this phenomenon [58]. Until recently, it was reasonable to assume that, with a correct prescription (dosage and administration), the artemisinin derivatives were universally effective against $P$. falciparum. Long-term eradication strategies must encompass drug treatment with either ACTs or low dose primaquine to achieve reductions in parasite transmission. Development of novel gametocidal drugs is clearly a priority to insure that drugs are available to achieve the "last mile" of malaria eradication [59].

\section{PK/PD profile matching will deter resistance to ACTs}

The principle problems in deterring the emergence of malaria drug resistance are associated with the use of malaria monotherapy or incomplete treatment. Not only are there well-known problems with drug compliance associated with patient treatment of malaria there are also issues of poor drug quality and access to malaria drugs in the marketplace without physician oversight. Broad resistance to all antimalarial drugs in various geographic regions, now including the artemisinins, has occurred historically [24-26], and therefore, the treatment of malaria disease by CT must employ an array of methods to find the most appropriate combination partner drugs for a particular geographic region. We have suggested the use of PK and PD parameters for selection of the most appropriate partner drugs, and there are some studies where this approach has been taken to find the best PK/PD match.

PK/PD matching between combination partners has been evaluated in a few studies. The artemisinin derivative, dihydroartemisinin (DHA), and MQ were investigated in zero healthy Thai males in a study designed as a three-way crossover study [60]. Patients in this study were distributed into three treatment groups dosed with three different combinations of DHA and mefloquine. In the first group, patients were administered a single oral dose of $300 \mathrm{mg}$ of DHA, the patients in the second group were given a single oral dose of $750 \mathrm{mg}$ of $\mathrm{MQ}$, and the patients in the third group were given $300 \mathrm{mg}$ of DHA and $750 \mathrm{mg}$ of MQ. The patients in all three groups tolerated their treatments well. Oral DHA was shown to be quickly absorbed and metabolized within 8-10 hours while MQ showed a different pattern. The absorption and 
clearance of MQ was slow compared to DHA, however, the absorption rate of MQ was faster when co-administered with DHA suggesting a synergistic process.

Pharmacodynamically, the combination of DHA and MQ resulted in a synergistic schizonticidal activity when tested ex vivo using blood from treated subjects. The maximum activity $\left(E_{\max }\right)$ of DHA was increased by a factor of two, and the $\left(E_{\max }\right)$ of MQ was increased by 20 fold compared with each drug alone. The area under effect-time curve (AUEC) of DHA was increased by factor of 4, and the AUEC of MQ was increased by a factor of two compared with each individual drug alone. The AUEC of MQ during the first 24 hours (AUEC ${ }_{0-24 h}$ ) was increased approximately 50-fold in the presence of DHA [61]

The dynamic profiles of both DHA and MQ were shown to coincide generally with their kinetic profiles in this study. The co-administration model for AS and MQ provided a more accurate estimation of the relative contributions of the benefit to therapy without any enhancement of adverse effects of each individual drug. The model-independent PD measures showed significant increases in the AUEC and the maximum response $\left(E_{\max }\right)$ of both DHA and MQ when they were given in combination compared with each individual drug alone. MQ was not shown to have a significant effect on the maximal effect of DHA, while DHA co-administration dramatically increased the $\mathrm{E}_{\max }$ of $\mathrm{MQ}$ [61].

The combination of artemether and lumefantrine is a new and very well tolerated oral antimalarial drug effective even against multidrug resistant falciparum malaria. The artemether component is absorbed rapidly and slowly biotransformed to dihydroartemisinin, and both the parent and metabolite were eliminated with half-lives of approximately 1 hour. Both artemether and DHA provide rapid reduction of the parasite biomass and quick resolution of symptoms. Lumefantrine has a half-life of 3-6 days with highly variable absorption dependent on co-administration with fat, which enhances absorption. The role of lumefantrine is to clear the few parasites that actually remain after artemether treatment, and lumefantrine plasma concentrations on day 7 (or the area under the curve) have been shown to correlate with treatment outcomes. [62, 63].

Traditionally, the selection of doses of artemisinin drugs in ACTs has been empirical in the absence of an established PK/PD relationship. PK/PD matching of artemisinins has been studied in detail particularly when artemisinins are administered in combination treatment. Based on PK/PD modeling, Hoshen et al. postulated that a small fraction of malaria parasites become cytostatic or dormant because of chemotherapeutic pressure. At this stage, the growth and development cycle of the parasite is halted, which results in the presence of parasites that are not drug susceptible to further dosing until the dormant state ends. This hypothesis suggests that the antimalarial activity of AS would be slower entailing either frequent doses or an extended period of treatment and surveillance. Based on modeling, the authors developed a strategy for selecting rational models for antimalarial chemotherapy [64].

Hoshen et al. [64] experimentally modeled pharmacokinetic and clinical data derived from a variety of regimens utilized to treat Thai patients treated with AS combined with mefloquine. The model assumed no PD interaction between AS and mefloquine, however, the parasites in many areas of Thailand have been shown in a variety of studies to already be resistant to 
mefloquine. The model produced suggested that treatment with either AS or mefloquine administered as monotherapy would fail, however, treatment with a daily dose of an AS-MQ drug combination for 3 days would be successful. The predictions of this study were on target with clinical outcomes as shown in a variety of studies examining the efficacy of the AS-MQ combination. This study yielded some very useful data, and the results merit a wider application for other drug combinations [64].

Angus et al. studied the dose-response relationship of AS used to treat acute falciparum malaria in adult Thai patients. This is the only study to date to report the dose response relationships of an artemisinin antimalarial agent used for patient treatment. In this study, patients received single oral doses of AS followed by mefloquine. The proper PD model was applied with dose, the measured drug concentrations of AS and its active metabolite DHA in the plasma, and parasite clearance values (the half maximal parasite clearance time or $\mathrm{PC}_{50}$, the time required for $90 \%$ of the parasites to be cleared or the $\mathrm{PC}_{90}$, and the overall parasite clearance time or PCT). There was considerable variance in the PC50 and PC90 values observed in patients dosed with mefloquine alone or with low doses of AS.

The $\mathrm{EC}_{50}$ required to achieve the half-maximal parasite clearance $\left(\mathrm{PC}_{50}\right)$ was shown to be 0.7 $\mathrm{mg} / \mathrm{kg}$, and the dose required to achieve $90 \%$ parasite clearance was shown to be $1.2 \mathrm{mg} / \mathrm{kg}$ (the $\mathrm{PC}_{90}$ ), and a dose of $1.4 \mathrm{mg} / \mathrm{kg}$ was required to achieve $100 \%$ parasite clearance (PCT). The $\mathrm{E}_{\max }$ was achieved at 28.6 hours, and the EC50 was estimated to be $1.6 \mathrm{mg} / \mathrm{kg}$. These results suggest that a dose of AS of $2 \mathrm{mg} / \mathrm{kg}$ is the lower limit of a maximum effective dose. Current practice includes a dose of $4 \mathrm{mg} / \mathrm{kg}$ of AS per day over a 3-day period in combination with a partner drug. This dose has been shown to be both safe and effective for treatment of uncomplicated falciparum malaria. For the artemisinin compounds, it is not clear which pharmacokinetic parameters is the most relevant related to a pharmacodynamic outcome. Additional studies will be needed to understand the relationship between concentration and effect for these compounds [65].

For the majority of antimalarials, parasite killing is not known to be strictly dependent on sustaining drug concentrations above the minimum inhibitory concentration or MIC. Therefore, getting a good match between pharmacokinetic and pharmacodynamic profiles to avoid the emergence of drug resistance is made more complex by the relationship between the nature of the antiparasitic action of a drug and the length of time required to achieve parasite killing. These factors interact with the selective window of a prospective antimalarial drug, which defines the period where sub-therapeutic levels of drug are present. This period of selectivity should be covered by a partner drug to avoid drug resistance.

There are two possibilities here; parasite killing of an antimalarial drug is dependent on the time that drug concentrations are maintained above the MIC (which might be as long as several 48-hour parasite life cycles), or the antiparasitic activity of an antimalarial drug might be dependent on total drug exposure as measured by AUC. The period of time during terminal drug elimination when drug levels fall below the MIC is called the "selective window". This window has been defined for pyrimethamine where a single dose of drug selects drug-resistant parasites after a period of 52 days. The selective window has also been defined for lumefantrine where a single dose of drug results in lumefantrine resistant parasites after a period of 30 days 
[5]. The ACTs in current use and recommended by the World Health Organization include, AS-SP, AS-AQ, AS-MQ, AL and DP, which are currently the most popular ACTs on market. For all of these drug combinations, the partner drug lasts far longer than the artemisinin component, and drug resistance has already been observed for each partner drug in various regions of the world to different degrees. ACT partner drugs may protect against induction of resistance to artemisinin drugs, and artemisinin compounds may prevent selection of parasites initially that are drug resistant, however, once artemisinin compounds are cleared, the partner drug is left with no protection and parasites will be exposed to sub-therapeutic drug levels during this "selective window" period.

Models have been created to analyze the probabilities of selection of drug resistant parasites, and these models suggest selection of drug resistant parasites is dependent on the fraction of patients with residual drug present from recent infections and not on the probability of a new infection occurring during the drug clearance phase. Accordingly, transmission intensity may not have a direct effect on selecting for drug resistant parasites [7].

Understanding the pharmacokinetic and pharmacodynamic profiles associated with drugs in the post-treatment period is clearly important. While the lower transmission rates found in Asia result in decreased use of drugs, these patients do present with symptomatic infections that must be treated. This, in turn, results in exposure of malaria parasites in these regions to malaria drugs. In contrast, patients in Africa are semi-immune to malaria infections and they may harbor reservoirs of parasites that are not treated as frequently. This may protect the population from selection for drug resistant parasites. While the use of artemisinin combination therapy has provided benefits to reversing declines in efficacy of partner drugs such as mefloquine in Asia, there may be consequences to the use of mismatched antimalarial drugs together in combination not yet observed [66].

We cannot predict with any confidence the fate of artemisinin drugs in Africa or the fate of their partner drugs. It may be that artemisinin drugs can reverse resistance to partner drugs, similar to the outcome of AS-MQ in Asia, or it is possible that these partner drugs and artemisinin compounds may fail in areas of Africa with higher malaria transmission rates. Factors associated with drug resistance such as the interaction of pharmacodynamics, pharmacokinetics, malaria epidemiology, and immunology should be considered when designing the next generation of antimalarial combination treatments. Conducting drug trials over extended periods of time will be necessary to understand how these variables interact, and the use of molecular markers of drug resistance for combination partners will be essential to monitor the emergence of drug resistance early prior to the emergence of treatment failures. A recent development in Malawi regarding the use of chloroquine showed drug resistance to chloroquine had disappeared 10 years after the government withdrew this drug from use. This provides a novel opportunity to examine the question of how long ACTs can deter the reemergence of chloroquine resistance in this country [66]. Long-term studies of combination treatment with chloroquine or treatment with chloroquine analogues like piperaquine may assist in determining the best method of extending the life of existing drugs. [16, 19]. 


\section{Conclusion}

Artemisinins are the most important class of antimalarial agents in the world today. These drugs are widely used, particularly for treatment of multidrug -resistant $P$. falciparum malaria. The first-generation artemisinins have their limitations, which include clinical evidence with a confirmed molecular marker to $P$. falciparum poor oral bioavailability, and short half-lives. Second- and third-generation artemisinins will likely be less expensive with improved properties. ACTs are more effective in malarial patients than other antimalarials in their ability to overcome recrudescence and minimize noncompliance through short duration treatment periods. To date, no clinically significant drug interactions have been observed with artemisinin combinations, which still require further study. The pharmacokinetic profiles of the artemisinins have been evaluated extensively in animals but not as extensively in humans, which is an unmet need. These compounds exhibit variable PK profiles and lack established $\mathrm{PK} / \mathrm{PD}$ relationships to support defining rational dosage regimens for malaria treatment. Future efforts should focus on understanding all of the parameters required to conduct a valid PK/PD match between an artemisinin compound and an appropriate partner drug [67].

\section{Author details}

\section{Qigui Li" and Mark Hickman}

*Address all correspondence to: qigui.li.civ@mail.mil

Department of Drug Discovery, Experimental Therapeutics Branch, Military Malaria Research Program, Walter Reed Army Institute of Research, Silver Spring, USA

Material has been reviewed by the Walter Reed Army Institute of Research. There is no objection to its presentation and/or publication. The opinions or assertions contained herein are the private views of the authors and are not to be construed as official, or as reflecting the views of the Department of the Army or the Department of Defense.

\section{References}

[1] Hastings, I.M., Hodel, E.M. (2014) Pharmacological considerations in the design of anti-malarial drug combination therapies - is matching half-lives enough? Malar J. 13,62 .

[2] WHO, Guidelines for the treatment of malaria (2010) 2nd Ed. Geneva, World Health Organization. http://www.who.int/malaria/publications/atoz/9789241547925/en/ 
[3] WHO, Global Report on Antimalarial Drug Efficacy and Drug Resistance: 2000-2010. (2010) Geneva: World Health Organization. http://www.who.int/malaria/publications/atoz/9789241500470/en/

[4] Kremsner, P.G., Krishna, S. (2004) Antimalarial combinations. Lancet. 364(9430), 285-294.

[5] Nyunt, M.M., Plowe, C.V. (2007) Pharmacologic advances in the global control and treatment of malaria: Combination therapy and resistance. Clin Pharmacol Ther. 82, 601-605.

[6] Gogtay, N., Kannan, S., Thatte, U.M., Olliaro, P.L., Sinclair, D. (2013) Artemisininbased combination therapy for treating uncomplicated Plasmodium vivax malaria. Cochrane Database Syst Rev. 10, CD008492.

[7] Hastings, I.M., Watkins, W.M. (2006) Tolerance is the key to understanding antimalarial drug resistance. Trends Parasitol. 22, 71-77.

[8] Martinelli, A., Moreira, R., Ravo, P.V. (2008) Malaria combination therapies: Advantages and shortcomings. Mini Rev Med Chem. 8, 201-212.

[9] Srivastava, S., Sherman, C., Meek, C., Leff, R., Gumbo, T. (2011) Pharmacokinetic mismatch does not lead to emergence of isoniazid- or rifampin-resistant Mycobacterium tuberculosis but to better antimicrobial effect: a new paradigm for antituberculosis drug scheduling. Antimicrob Agents Chemother. 55, 5085-5089.

[10] Vugt, M.V., Wilairatana, P., Gemperli, B., Gathmann, I., Phaipun, L., Brockman, A., Luxemburger, C., White, N.J., Nosten, F., Looareesuwan, S. (1999) Efficacy of six doses of artemether-lumefantrine (benflumetol) in multidrug-resistant Plasmodium falciparum malaria. Am J Trop Med Hyg. 60, 936-942.

[11] Lefevre, G., Looareesuwan, S., Treeprasertsuk, S., Krudsood,S., Silachamroon, U., Gathmann, I., Mull, R., Bakshi, R. (2001) A clinical and pharmacokinetic trial of six doses of artemether-lumefantrine for multidrug-resistant Plasmodium falciparum malaria in Thailand. Am J Trop Med Hyg. 64, 247-256.

[12] Omari, A.A., Gamble, C., Garner, P. (2004) Artemether-lumefantrine for uncomplicated malaria: a systematic review. Trop. Med. Int. Health. 9, 192-199.

[13] Sinclair, D., Gogtay, N., Brand, F., Olliaro, P. (2011) Artemisinin-based combination therapy for treating uncomplicated Plasmodium vivax malaria. Cochrane Database Syst Rev. 7, CD008492.

[14] Falade, C.O., Ogundele, A.O., Yusuf, B.O., Ademowo, O.G., Ladipo, S.M. (2008) High efficacy of two artemisinin-based combinations (artemether-lumefantrine and artesunate plus amodiaquine) for acute uncomplicated malaria in Ibadan, Nigeria. Trop Med Int Health. 13, 635-643.

[15] Ndayiragije, A., Niyungeko, D., Karenzo, J., Niyungeko, E., Barutwanayo, M., Ciza, A., Bosman, A., Moyou-Somo, R., Nahimana, A., Nyarushatsi, J.P., Barihuta, T., Miz- 
ero, L., Ndaruhutse, J., Delacollette, C., Ringwald, P., Kamana, J. (2004) Efficacy of therapeutic combinations with artemisinin derivatives in the treatment of non-complicated malaria in Burundi. Trop Med Int Health. 9, 673-679.

[16] Hasugian, A.R., Purba, H.L, Kenangalem, E., Wuwung, R.M., Ebsworth, E.P., Maristela, R., Penttinen, P.M., Laihad, F., Anstey, N.M., Tjitra, E., Price, R.N. (2007) Dihydroartemisinin-piperaquine versus artesunate-amodiaquine: superior efficacy and posttreatment prophylaxis against multidrug-resistant Plasmodium falciparum and Plasmodium vivax malaria. Clin Infect Dis. 44, 1067-74.

[17] Plowe, C.V. (2007) Combination therapy for malaria: Mission accomplished? Clin Infect Dis. 44, 1075-1077.

[18] Smithuis, F., Kyaw, M.K., Phe, O., Aye, K.Z., Htet, L., Barends, M., Lindegardh, N., Singtoroj, T., Ashley, E., Lwin, S., Stepniewska, K., White, N.J. (2006) Efficacy and effectiveness of dihydroartemisinin-piperaquine versus artesunate-mefloquine in falciparum malaria: an open-label randomised comparison. Lancet. 367(9528), 2075-2085.

[19] Kamya, M.R., Yeka, A., Bukirwa, H., Lugemwa, M., Rwakimari, J.B., Staedke, S.G., Talisuna, A.O., Greenhouse, B., Nosten, F., Rosenthal, P.J., Wabwire-Mangen, F., Dorsey, G. (2007) Artemether-lumefantrine versus dihydroartemisinin-piperaquine for treatment of malaria: a randomized trial. PLoS Clin Trials. 2, e20.

[20] Zongo, I., Dorsey, G., Rouamba, N., Dokomajilar, C., Séré, Y., Rosenthal, P.J., Ouédraogo, J.B. (2007) Randomized comparison of amodiaquine plus sulfadoxine-pyrimethamine, artemether-lumefantrine, and dihydroartemisinin-piperaquine for the treatment of uncomplicated Plasmodium falciparum malaria in Burkina Faso. Clin Infect Dis. 45, 1453-1461.

[21] Grande, T., Bernasconi, A., Erhart, A., Gamboa, D., Casapia, M., Delgado, C., Torres, K., Fanello, C., Llanos-Cuentas, A., D'Alessandro, U. (2007) A randomised controlled trial to assess the efficacy of dihydroartemisinin-piperaquine for the treatment of uncomplicated falciparum malaria in Peru. PLoS One. 2, e1101.

[22] Janssens, B., van Herp, M., Goubert, L., Chan, S., Uong, S., Nong, S., Socheat, D., Brockman, A., Ashley, E.A., Van Damme, W. (2007) A randomized open study to assess the efficacy and tolerability of dihydroartemisinin-piperaquine for the treatment of uncomplicated falciparum malaria in Cambodia. Trop. Med. Int. Health. 12, 251-259.

[23] Mayxay, M., Thongpraseuth, V., Khanthavong, M., Lindegardh, N., Barends, M., Keola, S., Pongvongsa, T., Phompida, S., Phetsouvanh, R., Stepniewska, K., White, N.J., Newton, P.N. (2006) An open, randomized comparison of artesunate plus mefloquine vs. dihydroartemisinin-piperaquine for the treatment of uncomplicated Plasmodium falciparum malaria in the Lao People's Democratic Republic (Laos). Trop. Med. Int. Health. 11, 1157-1165.

[24] Dondorp, A.M., Nosten, F., Yi, P., Das, D., Phyo, A.P., Tarning, J., Lwin, K.M., Ariey, F., Hanpithakpong, W., Lee, S.J., Ringwald, P., Silamut, K., Imwong, M., Chotivanich, 
K., Lim, P., Herdman, T., An, S.S., Yeung, S., Singhasivanon, P., Day, N.P., Lindegardh, N., Socheat, D., White, N.J. (2009) Artemisinin resistance in Plasmodium falciparum malaria. N Engl J Med. 361, 455-467.

[25] WHO, Status Report on Artemisinin Resistance: January-2014.(2014) Geneva: World Health Organization. http://www.who.int/malaria/publications/atoz/status_rep_artemisinin_resistance_jan2014.pdf?ua=1

[26] Ariey, F., Witkowski, B., Amaratunga, C., Beghain, J., Langlois, A.C., Khim, N., Kim, S., Duru, V., Bouchier, C., Ma, L., Lim, P., Leang, R., Duong, S., Sreng, S., Suon, S., Chuor, C.M., Bout, D.M., Ménard, S., Rogers, W.O., Genton, B., Fandeur, T., Miotto, O., Ringwald, P., Le Bras, J., Berry, A., Barale, J.C., Fairhurst, R.M., Benoit-Vical, F., Mercereau-Puijalon, O., Ménard, D. (2014) A molecular marker of artemisinin-resistant Plasmodium falciparum malaria. Nature. 505(7481), 50-55.

[27] Giao, P.T., de Vries, P.J. (2001) Pharmacokinetic interactions of antimalarial agents. Clin Pharmacokinetics. 40, 343-373.

[28] Li, Q., Weina, P. (2011) Antimalarial in Drugs: Age of the Artemisinins. Edited by Qigui Li \& Peter Weina. Nova Science Publishers Inc, New York; 1st edition, August. pp. 111-194.

[29] Sisowath, C., Strömberg, J., Mårtensson, A., Msellem, M., Obondo, C., Björkman, A., Gil, J.P. (2005) In vivo selection of Plasmodium falciparum pfmdr1 86N coding alleles by artemether-lumefantrine (Coartem). J Infect Dis.191, 1014-1017.

[30] Hastings, I.M., Ward, S.A. (2005) Coartem (artemether-lumefantrine) in Africa: The beginning of the end? J Infect Dis. 192, 1303-1304.

[31] Hastings, I.M., Watkins, W.M., White, N.J. (2002) The evolution of drug-resistant malaria: The role of drug elimination half-life. Philos Trans R Soc Lond B Biol Sci. 357, 505-519.

[32] Cross, A.P., Singer, B. (1991) Modeling the development of resistance of Plasmodium falciparum to anti-malarial drugs. Trans R Soc Trop Med Hyg. 85, 349-355.

[33] Austin, D.J., White, N.J., Anderson, R.M. (1998) The dynamics of drug action on the within-host population growth of infectious agents: Melding pharmacokinetics with pathogen population dynamics. J Theor Biol. 194, 313-339.

[34] Watkins, W.M., Mosobo, M. (1993) Treatment of Plasmodium falciparum malaria with pyrimethamine-sulfadoxine: Selective pressure for resistance is a function of long elimination half-life. Trans R Soc Trop Med Hyg. 87, 75-78.

[35] Palmer, K.J., Holliday, S.M., Brogden, R.N. (1993) Mefloquine. A review of its antimalarial activity, pharmacokinetic properties and therapeutic efficacy. Drugs. 45, 430-475. 
[36] Wells, T.N., Alonso, P.L., Gutteridge, W.E. (2009) New medicines to improve control and contribute to the eradication of malaria. Nat Rev Drug Discov. 8, 879-891.

[37] Vijaykadga, S., Rojanawatsirivej, C., Cholpol, S., Phoungmanee, D., Nakavej, A., Wongsrichanalai, C. (2006) In vivo sensitivity monitoring of mefloquine monotherapy and artesunate-mefloquine combinations for the treatment of uncomplicated falciparum malaria in Thailand in 2003. Trop Med Int Health. 11, 211-219.

[38] Ezzet, F., Mull, R., Karbwang, J. (1998) Population pharmacokinetics and therapeutic response of CGP 56697 (artemether + benflumetol) in malaria patients. Br J Clin Pharmacol. 46, 553-561.

[39] van Vugt, M., Looareesuwan, S., Wilairatana, P., McGready, R., Villegas, L., Gathmann, I., Mull, R., Brockman, A., White, N.J., Nosten, F. (2000) Artemether-lumefantrine for the treatment of multidrug-resistant falciparum malaria. Trans $\mathrm{R}$ Soc Trop Med Hyg. 94, 545-548.

[40] Krishna, S., White, N.J. (1996) Pharmacokinetics of quinine, chloroquine and amodiaquine. Clinical implications. Clin Pharmacokinet. 30, 263-299.

[41] Swarthout, T.D., van den Broek, I.V., Kayembe, G., Montgomery, J., Pota, H., Roper, C. (2006) Artesunate + amodiaquine and artesunate + sulphadoxine-pyrimethamine for treatment of uncomplicated malaria in Democratic Republic of Congo: a clinical trial with determination of sulphadoxine and pyrimethamine-resistant haplotypes. Trop Med Int Health. 11, 1503-1511.

[42] White, N.J. (1987) Combination treatment for falciparum prophylaxis. Lancet. 1, 680681.

[43] Nosten, F., vanVugt, M., Price, R.N., Luxemburger, C., Thway, K.L., Brockman, A., McGready, R., ter Kuile, F., Looareesuwan, S., White, N.J. (2000) Effects of artesunate-mefloquine combination on incidence of Plasmodium falciparum malaria and mefloquine resistance in western Thailand: a prospective study. Lancet. 356(9226), 297-302.

[44] Noedl, H., Se, Y., Schaecher, K., Smith, B.L., Socheat, D., Fukuda, M.M. (2008) Artemisinin Resistance in Cambodia 1 (ARC1) study consortium: Evidence of artemisinin-resistant malaria in western Cambodia. N Engl J Med. 359, 2619-2620.

[45] Woodrow, C.J., Krishna, S. (2006) Antimalarial drugs: Recent advances in molecular determinants of resistance and their clinical significance. Cell Mol Life Sci. 63, 1586-1596.

[46] Brockman, A., Price, R.N., van Vugt, M., Heppner, D.G., Walsh, D., Sookto, P., Wimonwattrawatee, T., Looareesuwan, S., White, N.J., Nosten, F. (2000) Plasmodium falciparum antimalarial drug susceptibility on the north-western border of Thailand during five years of extensive use of artesunate-mefloquine. Trans R Soc Trop Med Hyg. 94, 537-544. 
[47] Price, R.N., Nosten, F., Luxemburger, C., van Vugt, M., Phaipun, L., Chongsuphajaisiddhi, T., White, N.J. (1997) Artesunate/mefloquine treatment of multi-drug resistant falciparum malaria. Trans. R. Soc. Trop. Med. Hyg. 91, 574-577.

[48] Price, R.N., Nosten, F., Luxemburger, C., ter Kuile, F.O., Paiphun, L., Chongsuphajaisiddhi, T., White, N.J. (1996) Effects of artemisinin derivatives on malaria transmissibility. Lancet. 347, 1654-1658.

[49] White, N.J. (1998) Preventing antimalarial drug resistance through combinations. Drug Resist Update. 1, 3-9.

[50] White, N. (1999) Antimalarial drug resistance and combination chemotherapy. Philos Trans R Soc Lond B Biol Sci. 354(1384), 739-749.

[51] Sigma-tau. (2010) Application for inclusion of dihydroartemisinin plus piperaquine (DHA/PPQ) fixed dose combination tablets in the 17th edition of the WHO model lists of essential medicines. http://www.who.int/selection_medicines/committees/ expert/18/applications/D_Piperaquine.pdf.

[52] Hastings, I. (2011) How artemisinin-containing combination therapies slow the spread of antimalarial drug resistance. Trends Parasitol. 27, 67-72.

[53] Czock, D., Keller, F. (2007) Mechanism-based pharmacokinetic-pharmacodynamic modeling of antimicrobial drug effects. J Pharmacokinet Pharmacodyn. 34, 727-751.

[54] Kay, K., Hastings, I.M. (2013) Improving pharmacokinetic-pharmacodynamic modeling to investigate anti-infective chemotherapy with application to the current generation of antimalarial drugs. PLoS Comput Biol. 9, e1003151.

[55] Dye, C., Williams, B.G. (1997) Multigenic drug resistance among inbred malaria parasites. Proc Biol Sci. 264(1378), 61-67.

[56] Piedade, R., Gil, J.P. (2011) The pharmacogenetics of antimalarial artemisinin combination therapy. Expert Opin Drug Metab Toxicol. 7, 1185-1200.

[57] Kyle, D.E., Webster, H.K. (1996) Postantibiotic effect of quinine and dihydroartemisinin derivatives on Plasmodium falciparum in vitro: implications for a mechanism of recrudescence. Abstracts of the XIVth International Congress for Tropical Medicine and Malaria; 1996. Abstract 0-22-6.

[58] Witkowski, B., Lelievre, J., Barragan, M.J., et al. (2010) Increased tolerance to artemisinin in Plasmodium falciparum is mediated by a quiescence mechanism. Antimicrob Agents Chemother. 54, 1872-1877.

[59] Nosten, F. (2010) Waking the sleeping beauty. J Infect Dis. 202, 1300-1301.

[60] Nosten, F., Luxemburger, C., ter Kuile, F.O., Woodrow, C., Eh, J.P., Chongsuphajaisiddhi, T., White, N.J. (1994) Treatment of multidrug-resistant Plasmodium falciparum malaria with 3-day artesunate-mefloquine combination. J Infect Dis. 170, 971-977. 
[61] Na-Bangchang, K., Tippawangkosol, P., Thanavibul, A., Ubalee, R., Karbwang, J. (1999) Pharmacokinetic and pharmacodynamic interactions of mefloquine and dihydroartemisinin. Int J Clin Pharmacol Res. 19, 9-17.

[62] Gordi, T., Xie, R., Jusko, W.J. (2005) Semi-mechanistic pharmacokinetic/pharmacodynamic modeling of the antimalarial effect of artemisinin. Br J Clin Pharmacol. 60, 594-604.

[63] White, N.J., van, Vugt, M., Ezzet, F. (1999) Clinical pharmacokinetics and pharmacodynamics of artemether lumefantrine. Clin. Pharmacokinet. 37, 105-125.

[64] Hoshen, M.B., Stein, W.D., Ginsburg, H. (2002) Mathematical modelling of malaria chemotherapy: combining artesunate and mefloquine. Parasitology. 124(Pt 1), 9-15.

[65] Angus, B.J., Thaiaporn, I., Chanthapadith, K., Suputtamongkol, Y., White, N.J. (2002) Oral artesunate dose-response relationship in acute falciparum malaria. Antimicrob Agents Chemother. 46, 778-782.

[66] Laufer, M.K., Thesing, P.C., Eddington, N.D., Masonga, R., Dzinjalamala, F.K., Takala, S.L., Taylor, T.E., Plowe, C.V. (2006) Return of chloroquine antimalarial efficacy in Malawi. N. Engl. J. Med. 355, 1959-1966.

[67] Gautam, A., Ahmed, T., Batra, V., Paliwal, J. (2009) Pharmacokinetics and pharmacodynamics of endoperoxide antimalarials. Curr Drug Metab. 10, 289-306. 
Chapter 2

\title{
Pharmacokinetic (PK) Characteristics in Critically III Patients
}

\author{
Mayuki Aibiki and Noriyasu Fukuoka \\ Additional information is available at the end of the chapter \\ http://dx.doi.org/10.5772/60619
}

\section{Introduction}

Recently, it was evidenced that application of the PK in critical patients has been growing. We reported previously that pharmacokinetic (PK) properties of 1) an aminoglycoside for Methicillin Resistance Staphylococcus Aureus (MRSA) and 2) an antithrombin preparation for DIC (disseminated intravascular coagulation) are remarkably different in critically ill patients from the normal condition $(1 / 2)$. Therefore, we need to recognize these aspects on the treatments for such patients. So in this chapter, we review PK profile along with our previous reports and update pharmacological concept for critically ill patients.

\section{Antibiotics}

\subsection{Background of $\mathrm{ABK}$, an aminoglycoside}

Arbekacin (ABK) is an aminoglycoside for methicillin-resistant Staphylococcus aureus (MRSA) [3]. The bactericidal effect of ABK depends on the peak serum concentrations like other aminoglycosides [4]. In this regard, several reports demonstrated antibiotic pharmacokinetics in burn or septic patients, in whom conventional dosing led to treatment failure due to the increased distribution volume $\left(\mathrm{V}_{\mathrm{d}}\right)$ of the antibiotics $[5,6]$. On the other hand, the maintenance dosage of antibiotics, especially aminogylcoside, should be adjusted according to the patients' CCR when the agent is administered to patients with chronic renal dysfunction [7]. Furthermore, such antibiotic adjustment has been performed even in patients with acute renal dysfunction followed by the conventional regulation [7]. Interestingly, $V_{d}$ in chronic renal failure patients does not increase in contrast to patients with acute renal dysfunction, as 
previously reported $[8,9]$. Thus, in critically ill patients with acute renal dysfunction, there would be certain differences in antibiotic dosing with those of chronic renal insufficiency.

\section{PK of ABK in critically ill patients}

We examined pharmacokinetics of ABK in critical patients with acutely lowered CCR as well as in healthy volunteers. The serum concentrations of ABK were measured using fluorescence polarization immunoassay in ten critically ill patients (patient group) and six healthy volunteers (control group). MRSA infection in patients in this study developed from 14 to 22 days after admission (Table 1). The patients were given ABK at the recommended dose for subjects with normal CCR: $2 \mathrm{mg} / \mathrm{kg}$ with $100 \mathrm{ml}$ normal saline for 30 minutes twice a day [10]. In six normal volunteers (control group), $\mathrm{ABK}$ at the same dosage as in the patient group was administered intravenously for 30 minutes. Data were analyzed with a two-compartment model program for ABK [10].

\begin{tabular}{|c|c|c|c|c|c|c|c|c|c|}
\hline \multicolumn{10}{|c|}{ Patient group } \\
\hline & Age & Gen. & BW & Ht. & Prim. Dis. & CCR & APACHE 11 & MRSA & Prog. \\
\hline & $(\mathrm{Y})$ & & (kg) & $(\mathrm{cm})$ & \multicolumn{3}{|c|}{$(\mathrm{ml} / \mathrm{min})$} & \multicolumn{2}{|c|}{ Develop (days) } \\
\hline 1. & 85 & $\mathrm{~F}$ & 45 & 140 & Pneumonia & 48.7 & 24 & 14 & surv. \\
\hline 2. & 64 & $\mathrm{~F}$ & 47 & 155 & $\mathrm{SAH}$ & 70 & 23 & 15 & surv. \\
\hline 3. & 55 & M & 50 & 155 & $\mathrm{ICH}$ & 50 & 24 & 15 & surv. \\
\hline 4. & 70 & M & 58 & 165 & Pneumonia & 40.3 & 22 & 16 & surv. \\
\hline 5. & 55 & M & 50 & 163 & TBI & 80 & 22 & 17 & surv. \\
\hline 6. & 89 & $\mathrm{~F}$ & 45 & 150 & $\mathrm{SAH}$ & 60 & 25 & 21 & surv. \\
\hline 7. & 50 & F & 70 & 150 & BURN (2W) & 44.2 & 20 & 14 & dead \\
\hline 8. & 50 & $\mathrm{M}$ & 68 & 165 & post CPR & 65.4 & 30 & 18 & dead \\
\hline 9. & 60 & $\mathrm{M}$ & 65 & 168 & TBI & 70 & 26 & 20 & surv. \\
\hline 10. & 86 & $\mathrm{~F}$ & 45 & 153 & Sev. Ac. Panc. & 53.1 & 35 & 22 & surv. \\
\hline \multicolumn{10}{|c|}{ Control group } \\
\hline & Age & Gen. & BW & $\mathrm{Ht}$ & CCR & & & & \\
\hline & $(\mathrm{Y})$ & & $(\mathrm{kg})$ & (cm) & $(\mathrm{ml} / \mathrm{min})$ & & & & \\
\hline 1. & 49 & M & 66.5 & 169 & 100 & & & & \\
\hline 2. & 40 & M & 76 & 172 & 95 & & & & \\
\hline 3. & 45 & M & 60 & 160 & 110 & & & & \\
\hline 4. & 41 & M & 67.5 & 166 & 95 & & & & \\
\hline
\end{tabular}




\begin{tabular}{|c|c|c|c|c|c|c|c|c|}
\hline \multicolumn{9}{|c|}{ Patient group } \\
\hline & Age & Gen. & BW & Ht. & Prim. Dis. & CCR & APACHE 11 & MRSA \\
\hline & $(\mathrm{Y})$ & & (kg) & $(\mathrm{cm})$ & & $(\mathrm{ml} / \mathrm{min})$ & & Develop (days) \\
\hline 5. & 32 & M & 59 & 163 & 106 & & & \\
\hline 6. & 48 & F & 53 & 169 & 98 & & & \\
\hline
\end{tabular}

Gen.: gender, BW: body weight, Ht: height, CCR: creatinine clearance, SOFA: score to describe organ dysfuction/failure at the time of MRSA infection developing, MRSA Develop: MRSA infection development day, SAH: subarachnoid hemorrhage, ICH: intra-cerebral hemorrhage, TBI: traumatic brain injury, CPR: cardiopulmonary resuscitation, Sev. Ac. Panc.: severe acute pancreatitis

Table 1. Demographic Data of patients of ABK study

\begin{tabular}{cccc}
\hline \multicolumn{2}{c}{-ABK Study- } & \\
\hline Patient group (N=10) & \multicolumn{2}{c}{ Control group (N=6) } \\
\hline Age & $66 \pm 15$ & $42 \pm 6$ & $\mathrm{p}<0.05$ \\
\hline Gender (F/M) & $5 / 5$ & $63 \pm 8$ & NS \\
\hline B.W. (kg) & $60 \pm 7$ & $165 \pm 5$ & NS \\
\hline Ht. (cm) & $153 \pm 15$ & $100 \pm 6$ & NS \\
\hline CCR (ml/min) & $61 \pm 15$ & & $\mathrm{p}<0.05$ \\
\hline
\end{tabular}

Abbreviations: F, female; M, male; BW, body weight; Ht, height; $C C R$, creatinine clearance. Data represent as mean \pm SD.

Table 2. Comparisons of demographic data between patient and control groups

CCR, which was calculated using Cockcroft and Gault's equation of the patient group (CCR: $61 \pm 15 \mathrm{ml} / \mathrm{min}$ ), was significantly lower than that of the control group (CCR: $100 \pm 6 \mathrm{ml} / \mathrm{min}$ ). Despite low CCR, the ABK dosage suitable for CCR of normal range did not increase the maximum serum level (Cmax) to the effective range in the patient group. Although there were no significant changes in the drug clearance (CL) between the groups, Vss/BW (the percentage of the distribution volume to body weight) of the patients was remarkably increased to $69 \pm 26$ as compared to the control group of $18.7 \pm 3.6 \%$ (Figure 1A). The transfer rate constant to peripheral compartment $\left(\mathrm{k}^{1 / 2}\right)$ in the patient group was much higher than in the control (Figure 1B). A significant correlation between CCR and CL was found in the control group, but not in the patient group (Figure 1C).

These results indicate that in critical patients with lowered CCR, even ABK dose for normal CCR subjects does not elevate its serum concentration to effective levels due to augmented $\mathrm{V}_{\mathrm{d}}$ caused by increased $\mathrm{k}_{1 / 2}$. The present results suggest that adjustment of the antibiotic dosing according to CCR, as recommended generally, further lowers $C_{\max }$, which results in ineffectiveness of the drug in the critically ill patients having reduced CCR. 

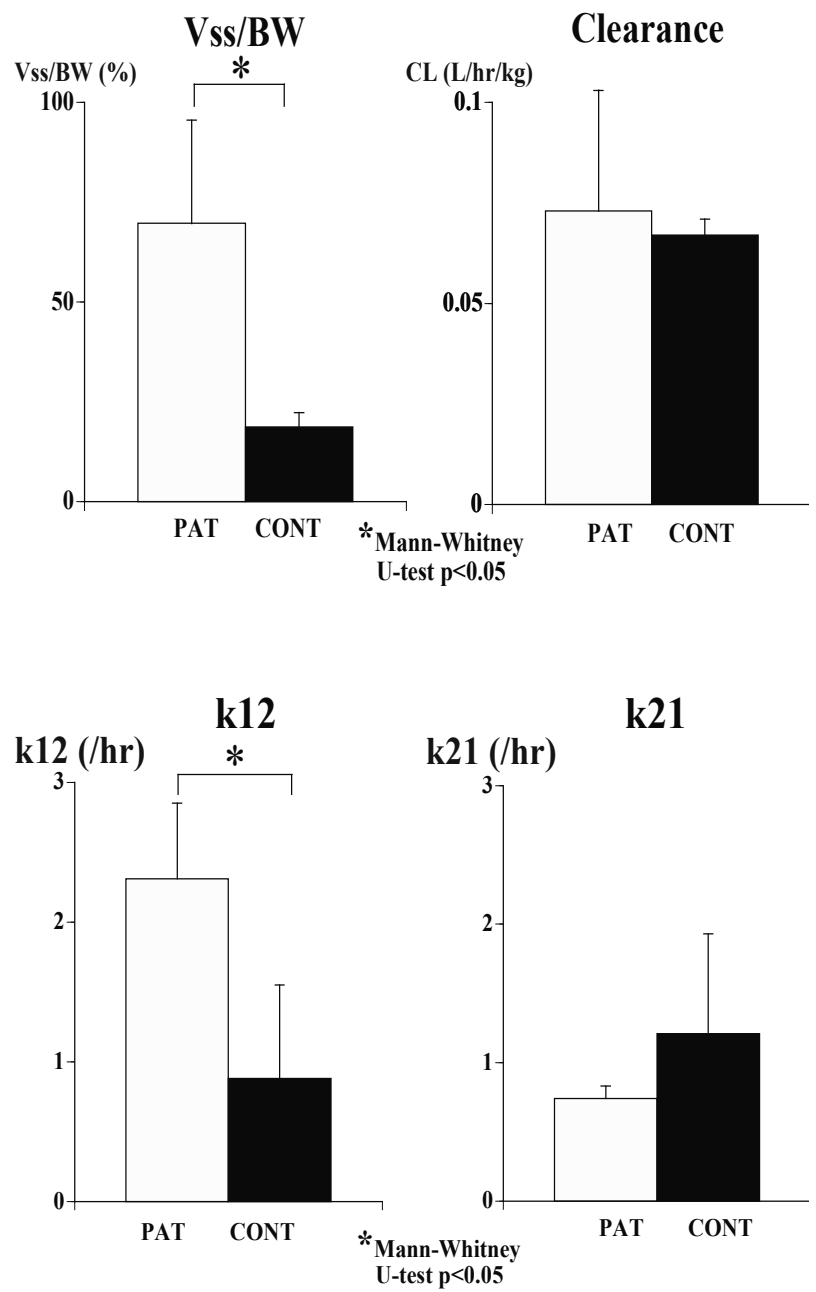

Figure 1. Vss/BW (the percentage of the distribution volume to 4 body weight) and the clearance between the groups

Figure 1A depicts the comparisons of Vss/BW (the percentage of the distribution volume to body weight) and the clearance between the groups. Remarkable inflation was found in $\mathrm{Vss} / \mathrm{Bw}$ of the patient group, but there were no significant differences in the clearance between the two groups. Figure 1B shows the comparisons of the elimination constants of the groups. $k^{1} / 2$ in the patients group is remarkably higher than that in the control group, but $k 21$ did not differ between the two groups. 


\subsection{Serum concentration changes of ABK in critically ill patients}

There are several reports demonstrating the distribution volume inflation of aminoglycoside in sepsis patients, indicating the importance of increasing the antibiotic dose in such conditions $[4,5]$. However, there has been limited information regarding the relationship between CCR and serum concentration of the agent in critically ill patients with increased distribution volume of aminoglycoside. It is generally known that adjusting the aminoglycoside dosage according to CCR is crucial for preventing untoward effects in patients with chronic renal dysfunction [7]. Furthermore, such antibiotic adjustment has been done even in patients with acute renal dysfunction by the conventional regulations [7]. However in chronic renal failure patients, the distribution volume of ABK does not elevate in contrast to that of those with acute renal failure $[8,9,10]$.

The current results showed that in critical patients with acutely decreased CCR, an ABK dose for normal CCR volunteers did not achieve effective levels of the agent. Moreover, pharmacokinetic analysis revealed remarkable increases both in $\mathrm{V}_{\mathrm{d}} / \mathrm{BW}$ and $\mathrm{k}_{1 / 2}$, but not in $\mathrm{k}_{21}$ as compared to the control. Aggressive fluid resuscitation for septic patients causes an inflation of the distribution volume for aminoglycosides [11]. Hypoalbuminemia may cause a shift of body water from the intravascular to the extravascular space [12]. Actually, this study showed a positive daily water balance as well as hypoalbuminemia in the patient group (data not shown). Thus, it is probable that the decreased $C_{\max }$ presented in this study is resulting from 1) augmented distribution volume, possibly resulting from edema, and 2) enhanced drug distribution from central to peripheral compartments.

\subsection{Pharmacokinetic differences between acute and chronic renal dysfunction}

In this study, we have shown $V_{d}$ increment as well as transfer rate constant enhancement in critically ill patients with lowered renal function. However, chronic renal failure patients have normal distribution volume of ABK $[8,9,10]$ as well as other aminoglycosides [13]. The CL of ABK correlates significantly with CCR in patients with chronic renal failure [8]. However, in the patients of this study, there were no correlations between the two parameters, as shown in Figure 2B. The pharmacokinetic condition differs greatly between acute and chronic renal dysfunctions. Thus, the CL of ABK did not estimate according to CCR in an acutely lowered renal dysfunction. We therefore need to reconsider dosing for aminoglycoside based on CCR in patients with acute renal dysfunction, who have augmented distribution volume and/or elevated vascular permeability. The present results also indicate that in critically ill patients with reduced renal function, the requiring dosage of $\mathrm{ABK}$ for obtaining an effective level will be higher than the recommended dose for subjects with intact renal function. Thus, in critically ill patients receiving $\mathrm{ABK}$, therapeutic drug monitoring is very important, not only for avoiding the side effects, but also for obtaining adequate effects from the agent.

Figure 2A demonstrates the correlation between $\mathrm{Cmax}$ and $\mathrm{CCR}$ in each group. Figure 2B shows the correlations of the clearance and CCR in each group. Note that although there was no significant correlation between Cmax and CCR in either group (Figure 2A), a significant correlation between clearance and CCR was observed only in the control group (Figure 2B). 


\section{Cmax and CCR}
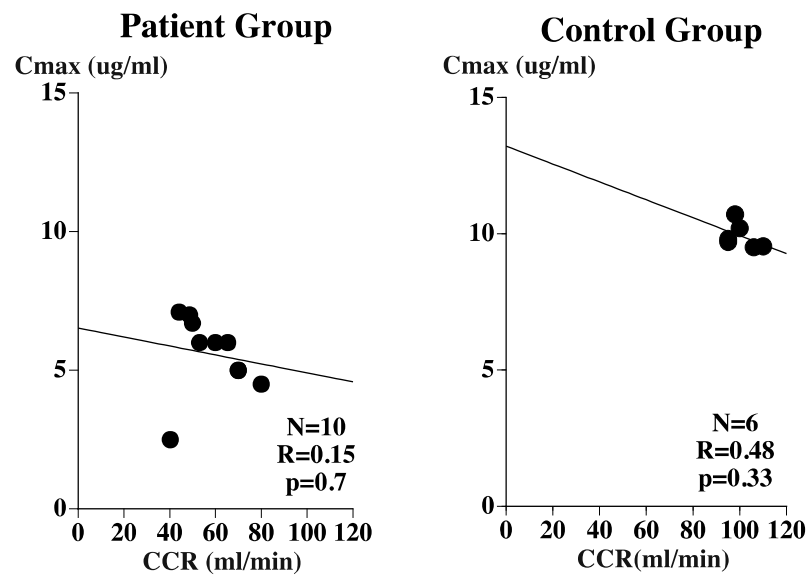

\section{Clearance and CCR}
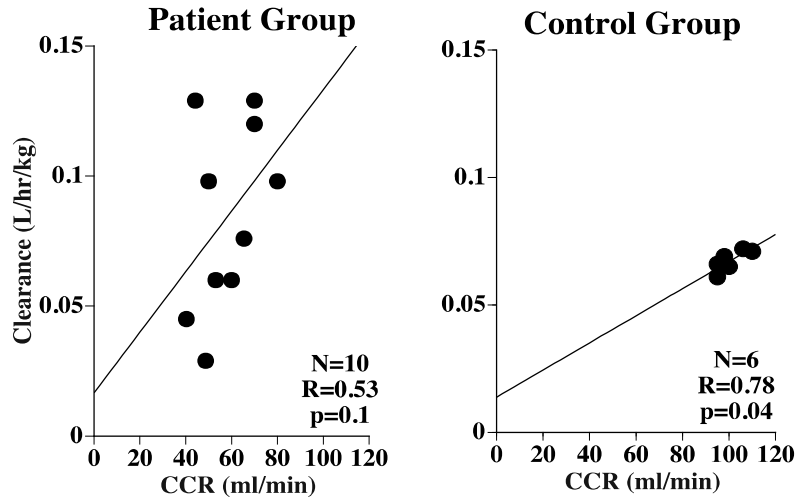

Figure 2. ABK Cmax and CCR; Drug Clearance and CCR

\section{Clinical applications}

Once-daily dosing is now widely used in aminoglycoside administration [14]. This method may be reasonable for obtaining an effective level of aminoglycoside, but a concern remains as to whether the concentration can be maintained below the toxic level of the agent. It has been reported that a therapeutic drug monitoring group receiving significantly greater doses of aminoglycosides than a once-daily group experience a significantly lower incidence of nephrotoxicity [15]. This recent report suggests the importance of therapeutic drug monitoring during the administration of aminoglycosides in each case. In our previous study, remarkable changes in the body fluid condition or drug pharmacokinetics were found in critically ill patients. Thus, we need to perform a pharmacokinetic analysis to estimate the appropriate 


\section{Two Compartment Model}

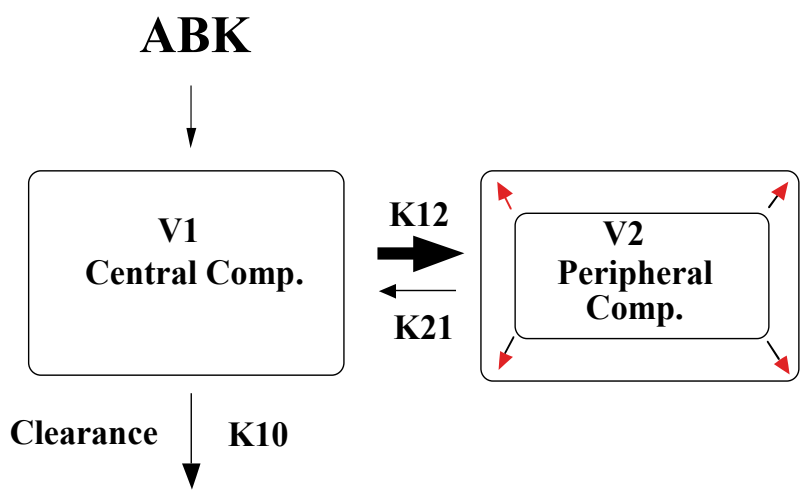

Figure 3. A possible mechanism for failure to obtain an effective level of ABK in critically ill patients. Note that there is an augmentation of the distribution volume (V2) as well as an increase in the elimination rate constant ( $\left.\mathrm{k}^{1 / 2}\right)$ of moving from the central to peripheral compartments.

dosage for administration of aminoglycosides, especially in critically ill patients with lowered renal function.

\section{Other antibiotics (vancomycin, daptomycin and barbapenums)}

\subsection{Vancomycin}

Recent study using a one-compartment model reported that, in septic patients receiving renal replacement therapy (RRT), mean vancomycin clearance $(\mathrm{CL})$ and $\mathrm{V}_{\mathrm{d}}$ were $2.9 \mathrm{~L} / \mathrm{h}$, $0.8 \mathrm{~L} / \mathrm{kg}$, respectively. Normal ranges of $\mathrm{CL}$ and $\mathrm{V}_{\mathrm{d}}$ are $9.1 \mathrm{~L} / \mathrm{h}, 0.4$ to $1 \mathrm{~L} / \mathrm{kg}$ [16]. However, no covariates were found to explain changing these parameters. Furthermore, CL did not correlate with RRT, which might be due to the multiple confounders known to influence antibiotic dosing in this setting. These data indicate the challenges of explaining pharmacokinetics in critically ill patients receiving RRT and emphasize a need for therapeutic drug monitoring in this setting [17].

Dosing of vancomycin (VAN) according to glomerular filtration rate (GFR) is an issue in critically ill patients. Recent paper shows very interesting findings as follows: seventy-eight adult patients admitted in intensive care unit received a $15 \mathrm{mg} / \mathrm{kg}$ loading dose of VAN followed by a continuous infusion at the rate of $30 \mathrm{mg} / \mathrm{kg} /$ day. Serum concentrations were measured 48 hours later and the dose was adjusted to obtain a target concentration ranging from 20 to $25 \mathrm{mg} / \mathrm{l}$. GFR was estimated by measured creatinine clearance (CLCR), Cockcroft, Modification of Diet in Renal Disease and Chronic Kidney Disease Epidemiology Collaboration (CKD-EPI) equations. The first dosage needed to be increased in $51 \%$ of all patients and 
in $84 \%$ of trauma patients, but decreased in $17 \%$ of patients. The closest relationship between clearances of VAN was found in CKD-EPI to GFR, but rather poor with the Cockcroft or Modification of Diet in renal Disease equation [18]. Although these findings indicate a caution on the VAN dosing using the latter two, this paper may show changes in distribution volume or vascular permeability of the agent in critically ill patients.

\subsection{Daptomycin}

Daptomycin is bactericidal against gram-positive bacteria, with peak-dependent effect but trough-dependent toxicity, but recommended dosing during continuous veno-venous hemodiafiltration (CVVHDF) remains unclear.

A study reported that in nine critically ill patients, undergoing CVVHDF due to acute renal failure and antimicrobial treatment, a pharmacokinetic analysis was done using an open twocompartment model. The total clearance was $6.1 \pm 4.9 \mathrm{~mL} / \mathrm{min}$, and the elimination half-life was $17.8 \pm 9.7 \mathrm{~h}$ (9 hours in normal subjects: [19]). The unbound fraction was $16 \pm 4.5 \%$ (protein binding: mean amount bound, $91.7 \%$, volume of distribution of $0.1 \mathrm{~L} / \mathrm{kg}$, [20]). Accumulation occurred if daptomycin was given every $24 \mathrm{~h}$. Simulation of $8 \mathrm{mg} / \mathrm{kg}$ daptomycin given every $48 \mathrm{~h}$ resulted in adequate levels without accumulation. So, the authors of this study recommend $8 \mathrm{mg} / \mathrm{kg}$ daptomycin every $48 \mathrm{~h}$ in patients on CVVHDF and therapeutic drug monitoring (TDM) [21].

In a prospective study, the pharmacokinetics (PK) of daptomycin after initial bolus $(8 \mathrm{mg} / \mathrm{kg}$ of body weight) and multiple intravenous doses ( $4 \mathrm{mg} / \mathrm{kg}$ body weight) at a stable state were determined for serum concentrations in critical patients treated with continuous hemodialysis (CVVHD). Subjects with normal renal function received one dose of $4 \mathrm{mg} / \mathrm{kg}$ body weight of daptomycin. Approximately $40 \%$ of the daptomycin dose administered was eliminated by CVVHD, so that, plasma half-lives of the agent in patients were 2 - 3 times longer as compared to normal controls. The dosing method of $4 \mathrm{mg} / \mathrm{kg}$ body weight of daptomycin given to patients with CVVHD every $48 \mathrm{~h}$ could not achieve effective plasma levels of daptomycin. Doses of more than and equal to $4 \mathrm{mg} / \mathrm{kg}$ every $24 \mathrm{~h}$ are necessary in CVVHD patients to assure plasma daptomycin levels [21]. These two studies show heterogeneity among critical patients treated with CVVHD or CVVHDF and also indicate an importance of TDM in each patient.

\subsection{Carbapenum (doripenem; meropenem)}

Doripenem is a carbapenem with limited data for dosing during CHDF in critically ill patients. A recent study determined the pharmacokinetics of doripenem in twelve septic adult patients with acute kidney injury (AKI) undergoing CHDF using a two-compartment model. The patients received $500 \mathrm{mg}$ of intravenous doripenem every $8 \mathrm{~h}$ for $60 \mathrm{~min}$. The median blood, dialysate and replacement fluid rates were 200,1000 and $1000 \mathrm{~mL} / \mathrm{h}$, respectively. The mean value for total doripenem clearance was $4.46 \mathrm{~L} / \mathrm{h}$ (normal range: $1 / 2$ to $16 \mathrm{~L} / \mathrm{h}$, [22]) and volume of distribution was $0.63 \mathrm{~L} / \mathrm{kg} \mathrm{BW}$ (normal range: 0.23 to $0.35 \mathrm{~L} / \mathrm{kg} \mathrm{BW}$, [22]). Doripenem elimination by CHDF depended on the rate filtration and contributed from $30 \%$ to $37 \%$ of total clearance. An intravenous dose of $500 \mathrm{mg}$ every $8 \mathrm{~h}$ obtained an MIC up to $4 \mathrm{mg} / \mathrm{L}$, which seems enough in PK/PD analyses for all patients. A dose of $500 \mathrm{mg}$ intravenously every $8 \mathrm{~h}$ was appropriate for our CVVHDF settings for infections caused by susceptible bacteria [23]. 
A recent randomized crossover study examined whether in ten critically ill patients, a prolonged 3-hour infusion of meropenem $500 \mathrm{mg}$ achieves an equivalent proportion of time above the MIC (\%TMIC) to that of meropenem 1000mg given over 30 minutes. Meropenem was administered as a $1000 \mathrm{mg}$, 30-minute infusion or as a $500 \mathrm{mg}$, 3-hour infusion. Serial plasma concentrations for each dosing were determined, thereby comparing \%TMIC at different MICs (the percentage of time above its MIC). For low MICs ( $\leq 2 \mathrm{mg} / \mathrm{L}$ ), both regimens attained a $\%$ TMIC $>40 \%$ in all patients. For an MIC of $4 \mathrm{mg} / \mathrm{L}$, this target was attained in all but one patient, but with an MIC of $8 \mathrm{mg} / \mathrm{L}$, three patients in each group had a $\% \mathrm{TMIC}<40 \%$. There was significant variability in the pharmacokinetic and hence the pharmacokinetic-pharmacodynamic parameters between individuals. Several patients had elevated creatinine clearances and, with both regimens, their target attainment was poor. Meropenem at $1000 \mathrm{mg}$ over 30 minutes achieved a similar \%TMIC to meropenem at 500mg given over 3 hours. Meropenem pharmacokinetics was highly variable from individual to individual [24].

\section{Biological preparations}

\subsection{Antithrombin (AT) agent}

\subsubsection{Summary of $A T$}

Decreased serum albumin levels suggest heightened vascular permeability in critically ill patients. In such a situation, plasma antithrombin (AT) may also decrease due to the leakage because albumin's molecular weight of 63,000 is similar to AT 56,000. But another mechanism of decreasing AT during DIC could be AT consumption, which could be estimated by the levels of plasma thrombin-antithrombin complex (TAT). So, we measured serum albumin levels and plasma TAT in patients with DIC before and after AT administration. The levels of serum albumin prior to AT administration correlated with pre- and post-administered AT activity, but not with TAT levels (Figure 1 (AT)). With no correlations of TAT levels, the trough levels of AT activity on the third day increased significantly. In patients with serum levels of albumin less than $2.5 \mathrm{~g} / \mathrm{dl}$, the trough levels of AT activity on the third day were significantly lower than those showing higher levels of serum albumin. The half-life duration of AT preparation in the distribution phase in the acute patients was shortened to less than $1 / 3$ of the value reported in congenital AT deficiency, suggesting increased vascular permeability in the acute patients. The distribution volume of the agent increased remarkably compared to the previous control. Therefore, we need to pay attention to the characteristic PK profiles of increased vascular permeability as well as distribution volume observed in critical patients even on the administration of AT preparation, a high molecular weight agent.

\subsubsection{Background of AT}

Antithrombin III (AT) has anti-coagulatory as well as anti-inflammatory effects [25, 26]. According to subgroup analyses of the Kybersept study data [27, 28], prognosis in septic patients with disseminated intravascular coagulation (DIC) might be improved with AT administration. 
Plasma thrombin-antithrombin complex (TAT) is a marker of intravascular thrombin formation, reflecting enhancement of intravascular coagulation [29]. In vitro thrombin application itself increases the permeability of endothelial cell monolayer [30]; however, it is still unknown whether AT levels after AT administration would change depending on TAT levels in patients with DIC.

On the other hand, albumin leakage from the capillaries is a more important mechanism for the decrease in serum albumin levels than its depressed synthesis during sepsis, where vascular permeability is enhanced [31,32,33]. Since the molecular weight of AT is similar to albumin, it is likely to expect that in critical conditions including sepsis, not only albumin but also AT would be permeable through the capillaries. We analyzed pharmacokinetic (PK) properties on AT preparation in order to define its PK characteristics in critically ill patients, because PK information for AT in such patients was not enough. The present results suggest that crucial mechanisms for AT reduction in critically ill patients with DIC might be a reduced synthesis and/or leakage of AT to extravascular space.

\subsection{Correlations among AT activity, TAT and albumin levels}

Figures of AT $4 \mathrm{~A}$ and $4 \mathrm{~B}$ show serum albumin levels prior to AT application correlated with plasma AT activities ( $\mathrm{rs}=0.72, \mathrm{p}=0.003)$, but not with TATs ( $\mathrm{rs}=0.38, \mathrm{p}=0.1$ ). The pre-administered serum albumin values also correlated with plasma AT trough activity on the third day after the initiation of AT preparation ( $\mathrm{rs}=0.76, \mathrm{p}=0.001)$.

As shown in Figures 4A and B, serum albumin levels before AT administration moderately correlated with serum AT levels ( $\mathrm{rs}=0.72, \mathrm{p}=0.003$ ), but TAT did not. The pre-administered serum albumin levels were also significantly correlated with AT trough activity on the third day after the treatment ( $\mathrm{rs}=0.76, \mathrm{p}=0.001$ ). Pre-TAT, TAT levels before AT administration; PreAT, AT activities before AT administration; Pre-albumin, serum albumin levels before AT administration; Post-AT, AT trough levels on the third day after AT administration.

As shown in Figures of AT 5A and B, the levels of AT trough activity on the third day elevated significantly in patients irrespective of TAT levels $15 \mu \mathrm{g} / \mathrm{l}$, a level of which was moderately elevated (normal level $<4 \mu \mathrm{g} / \mathrm{l}$ ). However, AT trough levels on the third day in patients with serum albumin levels $\leq 2.5 \mathrm{~g} / \mathrm{dl}$ were significantly lower than those with such levels higher than and equal to $2.5 \mathrm{~g} / \mathrm{dl}$.

As demonstrated in Figure 5A, AT trough levels on the third day increased significantly regardless of TAT levels of $15 \mu \mathrm{g} / \mathrm{l}$ (normal level $<4 \mu \mathrm{g} / \mathrm{l}$ ). As shown in Figure 5B, in patients with serum albumin levels less than and equal to $2.5 \mathrm{~g} / \mathrm{dl}$, AT trough levels on the third day were significantly lower than those more than and equal to $2.5 \mathrm{~g} / \mathrm{dl}$.

\subsection{PK analysis for AT}

As demonstrated in Figure 6 of AT, AT activity and antigen levels correlated well (rs=0.91, $\mathrm{p}=0.0001$ ). 

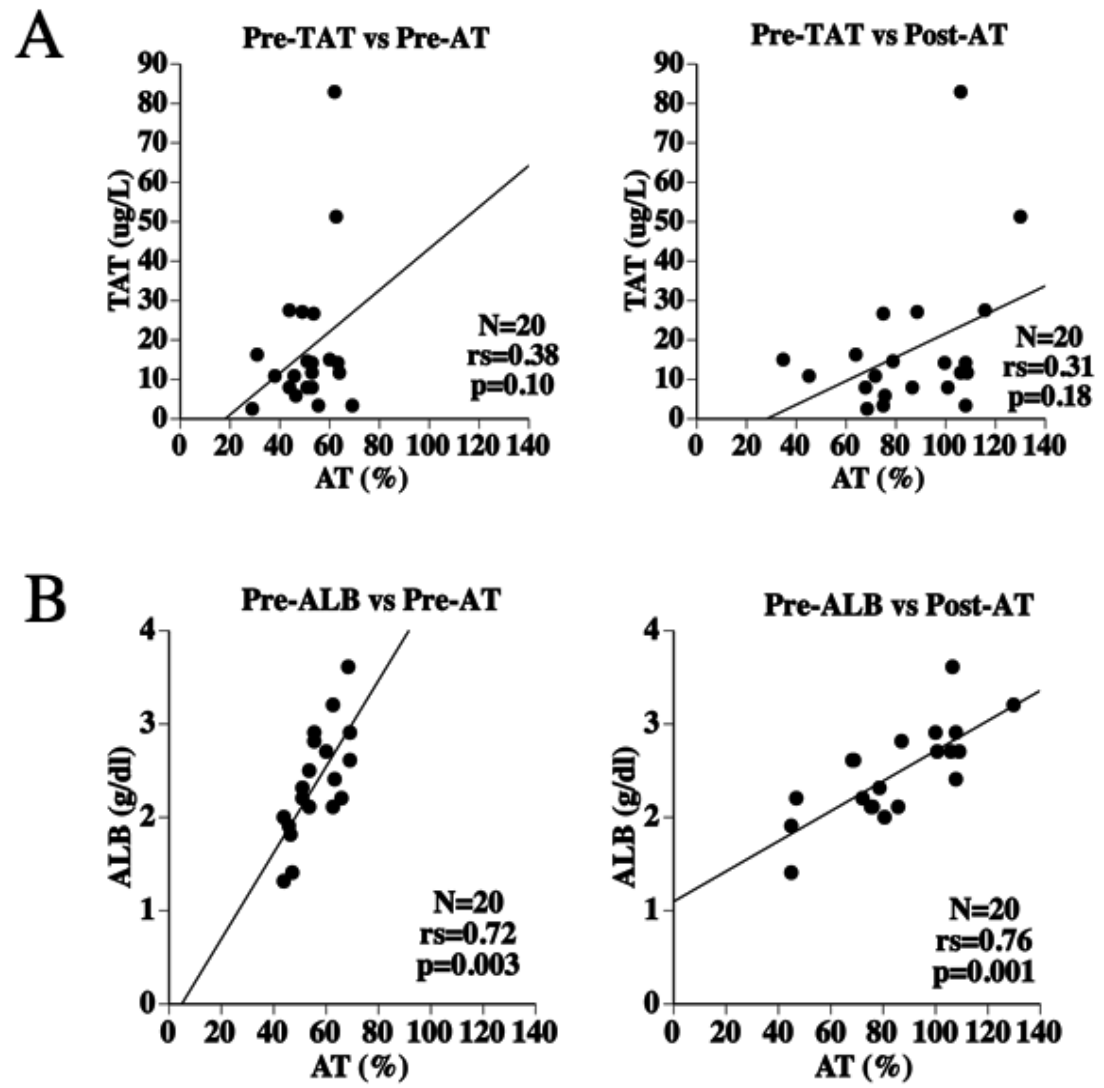

Figure 4. Panel A shows the correlations between pre-TAT and pre-AT levels (left), and post-AT levels (right). Panel B demonstrates the correlations between pre-albumin and pre-AT (left), and post-AT levels (right).

As shown in Figure 7A, the half-life of the distribution phase $\left(\alpha \mathrm{t}_{1 / 2}\right)$ in acute patients $(7.0 \pm 2$ hours) was markedly shortened as compared to that previously reported in patients with congenital AT deficiency ( $26.2 \pm 1.5$ hours). This result may suggest increased vascular permeability. However, the half-lives of the elimination phase $\left(\beta \mathrm{t}^{1 / 2}\right)$ in both groups were not different (congenital: $60 \pm 22$ versus acute: $62 \pm 7$ hours).

As shown in Figure 7A (left), the half-life of the distribution phase $\left(\alpha \mathrm{t}^{1 / 2}\right)$ in acute patients $(7 \pm 2$ hours, $\mathrm{N}=10$ ) was remarkably shortened as compared to the value of which was previously reported in patients with congenital AT deficiency $(26.2 \pm 1.5$ hours, $N=11)$. However, as demonstrated in Figure 7A (right), the half-lives of the elimination phase $\left(\beta \mathrm{t}^{1} / 2\right)$ in both groups did not differ (congenital: $60 \pm 22$ versus acute: $62 \pm 7$ hours). As demonstrated in Figure $7 \mathrm{~B}$, the distribution volume of the agent in the acute disease group inflated significantly to $6.9 \pm 3.3 \mathrm{~L}$ as compared to the previous control of $2.3 \pm 0.4 \mathrm{~L}$. Figure $7 \mathrm{~B}$ demonstrated that the distribution volume of the agent in the acute group increased remarkably to $6.9 \pm 3.3 \mathrm{~L}$ from the previous control of $2.3 \pm 0.4 \mathrm{~L}$. 


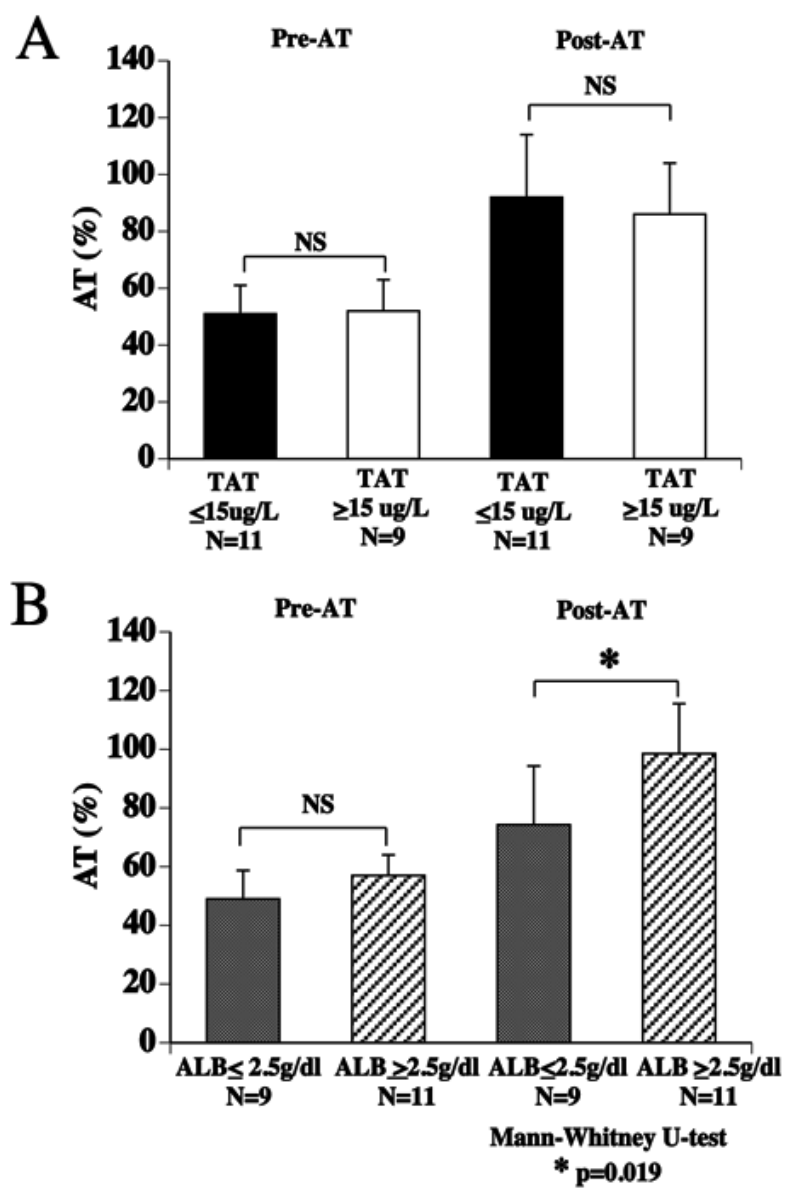

Figure 5. Comparisons of AT levels depending on TAT or Serum Albumin level in critically-ill patients

\subsection{Relations of AT activity and TAT or albumin levels}

TAT is a sensitive marker of thrombin production in the circulating blood, suggesting an augmented intravascular coagulation [34]. Intravascular production of thrombin may cause an enhanced vascular permeability [30]. Thus, it is likely that increased TAT levels could estimate AT leakage resulting from heightened vascular permeability. However, in this study, we found no correlations between TAT and AT activity in the pre-administration phase in critical patients with DIC. Furthermore, TAT is believed to be uncorrelated with AT activity because the total amount of AT in the body is so enormous that AT levels would not decrease even after remarkable TAT production [35]. Therefore, the present results confirm the data of the previous paper [35].

It was unknown whether AT activity after AT preparation would change depending on the levels of TAT if vascular permeability was enhanced. If TAT is a potential indicator of showing increased vascular permeability, TAT levels before AT administration would be well corre- 


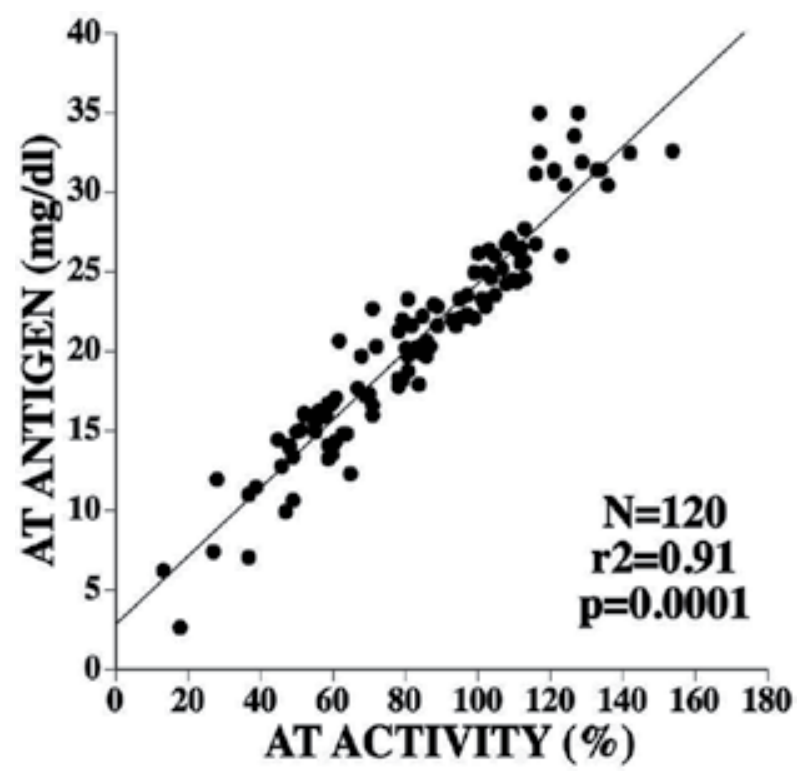

Figure 6. Correlations between AT activity and antigen are shown. A good correlation is obtained between the two parameters ( $\mathrm{rs}=0.91, \mathrm{p}=0.0001)$.

lated with AT activity after the administration of AT preparation. But, we demonstrated in this study that TAT levels before AT administration are not correlated even with postadministered AT activities. Therefore, it is possible that high TAT levels does not mean increased vascular permeability in clinical settings. However, TAT has a short half-life time (approximately 7 minutes) in the circulating blood [36], whereas AT has a much longer halflife period: approximately 24 hours in congenital AT deficiency in a non-acute state [37] as well as in normal subjects [38], and even in the acute state as presented in this study approximately 7 hours. So, we might be able to speculate that this difference in half-life time between TAT and AT activity is an explanation for the low correlation co-efficient as clarified in this study.

Serum albumin levels decreased due to its reduced synthesis and/or enhanced consumption during sepsis [31,32,33], frequently involving DIC. Albumin leakage from the capillaries is a factor more crucial for albumin reduction during sepsis [31]. Because the molecular weight of AT $(56,000)$ is smaller than that of albumin $(63,000)$, it is likely that during sepsis, albumin and AT could pass through the capillaries into the extravascular space. In the current study, AT levels altered with regard to serum albumin levels. Further, when the serum albumin level was lower than $2.5 \mathrm{~g} / \mathrm{dl}$, AT activities after administration was significantly lower as compared to those when the serum albumin level was over $2.5 \mathrm{~g} / \mathrm{dl}$. Therefore, it is possible that the serum albumin level could be a reliable index for estimating changes in the post-administration AT levels in critical patients with DIC. Furthermore, this study supports a previous paper [35] that in septic patients with DIC, AT could be decreased by enhanced vascular permeability rather than its consumption. 
A
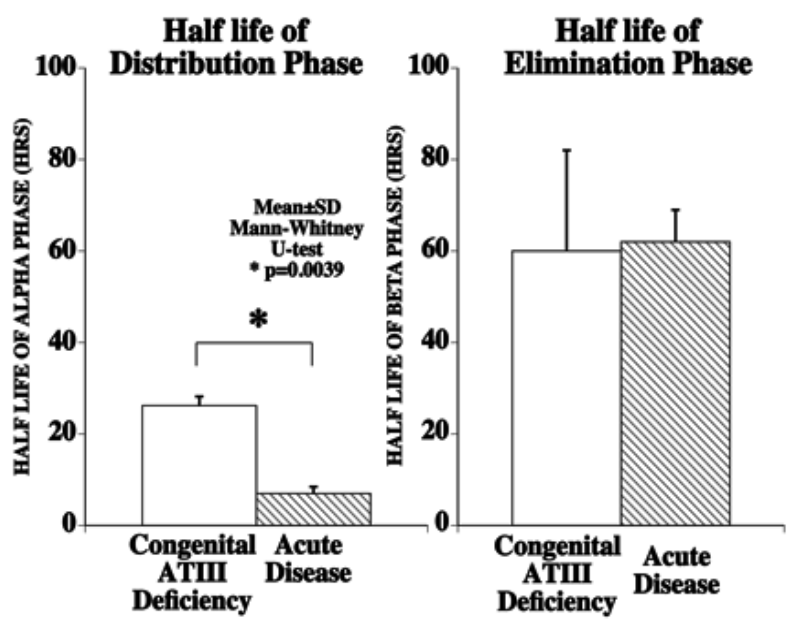

B

\section{Distribution Volume (VD) in Congenital and Acute Groups}

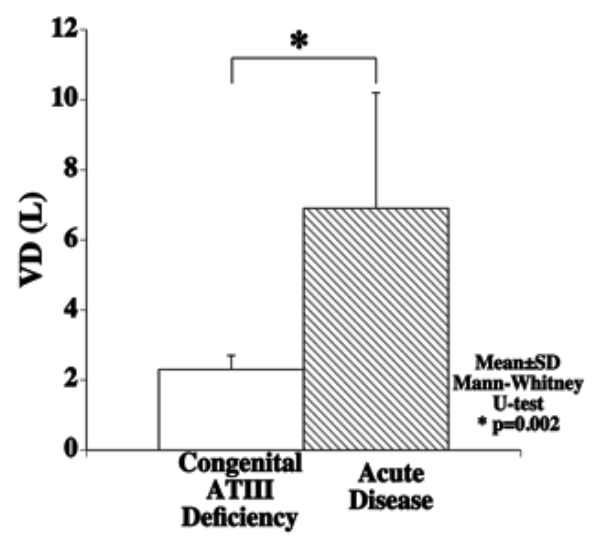

Figure 7. A: Half life of AT (elimination and distribution phases) in each condition; B: Comparison of distribution volume (VD) between congenital AT deficiency and acute disease

\subsection{Pharmacokinetics of AT agent in critical patients}

There are reports describing the pharmacokinetic analysis of AT agent in critical patients [39, 40]. However, a previous paper included several limitations: for example, the selection of a one-compartment model for AT agent in sepsis patients [40]. It is well known that AT distributes to the intra-vascular as well as extra-vascular spaces [41], which obliges us to choose a two-compartment model for this study. Furthermore, we found that changes in AT activity showed a biphasic decay curve after AT administration (Figure 6).

We have demonstrated clearly in this study that the half-life of the distribution phase in the patient group was shortened remarkably as compared to that previously reported in congenital AT deficiency, suggesting increased vascular permeability in the acute patients here. Further- 
more, the distribution volume in the patient group was inflated much more than that of the previous controls. We have shown for the first time that in critical patients with DIC, changes in AT can be predicted by serum albumin levels before AT administration, but not by TAT. These findings might be explained, at least in part, by the shortened AT half-life of the distribution phase and increased distribution volume observed in the critical patients in this study.

\subsection{Limitations of AT study}

As a control, we referred data from a previous paper describing AT pharmacokinetic analysis in congenital AT deficiency in a non-acute state [37], in which two-compartment analysis was used same way as our current study. It is likely that this congenital disease in non-acute state was not complicated by either an increase in vascular permeability or distribution volume [39]. We therefore selected the previously published data as a control for this study. However, further research is required to define any age-dependent changes in the PK profile of AT preparation because there was a significant difference in age between acute disease and congenital groups.

This study has several limitations in the variety of the diseases as well as number of patients, but all patients were complicated with DIC. Furthermore, we have found discrepancies between AT and TAT, and vascular permeability with increased distribution volume in the patients of this study. However, we need to clarify each pathological profile in critically ill patients with different pathology in the future.

\section{Conclusions}

We described in this chapter that in critically ill patients even with low CCR, the ABK dose for normal CCR subjects does not elevate its serum concentration to effective levels because of augmented distribution volume and increased vascular permeability. Furthermore, we reported in critical patients with DIC, shortened elimination half-life and increased distribution volume AT, a preparation of large molecular weight. From the view of pharmacokinetic profile, these findings presented here should be considered on the administration of not only antibiotics but also biological agents to the critically ill patients.

\section{Author details}

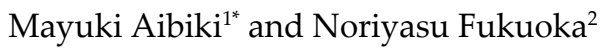

*Address all correspondence to: aibiki@m.ehime-u.ac.jp

1 Ehime University, Graduate School of Medicine, Ehime, Japan

2 Department of Pharmacology, Nihon University, Chiba, Japan 


\section{References}

[1] Fukuoka N, Aibiki M. Recommended dose of arbekacin, an aminoglycoside against methicillin-resistant Staphylococcus aureus, does not achieve desired serum concentration in critically ill patients with lowered creatinine clearance. J Clin Pharm Ther. 33: 521-527, 2008.

[2] Aibiki M, Fukuoka N, Nishiyama T, Maekawa S, Shirakawa Y. Differences in antithrombin III activities by administration method in critical patients with disseminated intravascular coagulation: a pharmacokinetic study. Shock. 28: 141-147, 2007.

[3] Kak V, You I, Zervos MJ, Kariyama R, Kumon H, Chow JW. In-vitro synergistic activity of the combination of ampicillin and arbekacin against vancomycin-and highlevel gentamicin-resistant enterococcus faecium with the aph(2")-Id gene. Diagn Microbiol Infect Dis. 37: 297-299, 2000.

[4] Ristuccia AM, Cunha BA. An overview of amikacin. Ther Drug Monit. 7: 12-25, 1985.

[5] Nishizawa K, Ito J, Sugaya K, Nakajima M, Hirano M, Kimura A, Henmi H. Pharmacokinetics of AMK, ABK, VCM in Patients with Burns. Res TDM. 1/2: 165-166, 1995. (Abstract in English).

[6] Paepe PD, Belpaire FM, Buylaert WA. Pharmacokinetic and pharmacodynamic considerations when treating patients with sepsis and septic shock. Clin Pharmakinet. 41: 1135-1151, 2002.

[7] Pinder M, Bellomo R, Lipman J. Pharmacological principle of antibiotic prescription in the critically ill. Anaesth Intensive Care. 30: 134-144, 2002.

[8] Aronoff K. (eds.) Drug Prescribing in renal failure, 4th Ed., American College of Physicians, Churchill-Livingstone, 1999, pp. 195-236.

[9] Fillastre JP, Leroy A, Humbert G, Moulin B, Bernadet P, Josse S. Pharmacokinetics of habekacin in patients with renal insufficiency. Antimicrob Agents Chemother. 31: 575-577, 1987.

[10] Kumon H, Mizuno A, Nasu Y, Tsugawa M, Kishi M, Ohmori H. Pharmacokinetics of arbekacin in healthy volunteers and patients with renal insufficiency. Jap J Antibiotics. 37: 200-207, 1989 (Abstract in English).

[11] Yamamoto T, Koyama M, Nakagawa K. Phase-one clinical study on ABK. Chemotherapy. 34: 104-116, 1986 (Abstract in English).

[12] Shibasaki S, Mitomi N, Matsumoto T, Morishita N, Matsuno T, Fujii H, Tanigawara Y, Okumura K. Population pharmacokinetic analysis of arbekacin in Japanese. Res TDM. 17: 47-53, 2000 (Abstract in English). 
[13] Niemiec PW, Jr Allo MD, Miller CF. Effect of altered volume of distribution on aminoglycoside levels in patients in surgical intensive care. Arch Surg. 122: 207-212, 1987.

[14] Winston L, Benowitz N. Once-daily dosing of aminoglycosides: how much monitoring is truly required? Am J Med. 15: 239-240, 2003.

[15] Bartal C, Danon A, Schlaeffer F, Reisenberg K, Alkan M, Smoliakov R, Sidi A, Almog Y. Pharmacokinetic dosing of aminoglycosides: a controlled trial. Am J Med. 15: 194198, 2003.

[16] Rodvold KA, Blum RA, Fischer JH, Zokufa HZ, Rotschafer JC, Crossley KB, Riff LJ. Vancomycin pharmacokinetics in patients with various degrees of renal function. Antimicrob Agents Chemother. 32:848-852, 1988.

[17] Udy AA, Covajes C, Taccone FS, Jacobs F, Vincent JL, Lipman J, Roberts JA. Can population pharmacokinetic modelling guide vancomycin dosing during continuous renal replacement therapy in critically ill patients? Int J Antimicrob Agents. 41: 564$568,2013$.

[18] Conil JM, Georges B, Breden A, Ruiz S, Cougot P, Fourcade O, Saivin S. Estimation of glomerular filtration rate to adjust vancomycin dosage in critically ill patients: superiority of the chronic kidney disease epidemiology collaboration equation? Anaesth Intensive Care. 42: 178-184, 2014.

[19] Dvorchik BH, Brazier D, DeBruin MF, Arbeit RD. Daptomycin pharmacokinetics and safety following administration of escalating doses once daily to healthy subjects. Antimicrob Agents Chemother. 47: 1318-1323, 2003.

[20] Wenisch JM, Meyer B, Fuhrmann V, Saria K, Zuba C, Dittrich P, Thalhammer F. Multiple-dose pharmacokinetics of daptomycin during continuous venovenous haemodiafiltration. J Antimicrob Chemother. 67: 977-983, 2012.

[21] Khadzhynov D, Slowinski T, Lieker I, Spies C, Puhlmann B, König T, Uhrig A, Eggers K, Neumayer HH, Traunmüller F, Joukhadar C, Peters H. Plasma pharmacokinetics of daptomycin in critically ill patients with renal failure and undergoing CVVHD. Int J Clin Pharmacol Ther. 49: 656-665, 2011.

[22] Nandy P, Samtani MN, Lin R. Population pharmacokinetics of doripenem based on data from phase 1 studies with healthy volunteers and phase 2 and 3 studies with critically Ill patients. Antimicrob Agents Chemother. 54: 2354-2359, 2010.

[23] Roberts JA, Udy AA, Bulitta JB, Stuart J, Jarrett P, Starr T, Lassig-Smith M, Roberts NA, Dunlop R, Hayashi Y, Wallis SC, Lipman J. Doripenem population pharmacokinetics and dosing requirements for critically ill patients receiving continuous venovenous haemodiafiltration. J Antimicrob Chemother. 69: 2508-2516, 2014. 
[24] Langan KM, Jacob J, Li J, Nation RL, Bellomo R, Howden B, Johnson PD. Pharmacokinetics of short versus extended infusion meropenem dosing in critically ill patients: a pilot study. Crit Care Resusc. 16: 190-196, 2014.

[25] Dickneite G. Antithrombin III in animal models of sepsis and organ failure. Semin Thromb Hemost. 24: 61-69, 1998.

[26] Leitner JM, Firbas C, Mayr FB, Reiter RA, Steinlechner B, Jilma B. Recombinant human antithrombin inhibits thrombin formation and interleukin-6 release in human endotoxemia. Clin Pharmacol Ther. 79: 23-34, 2006.

[27] Wiedermann CJ, Hoffmann JN, Juers M, Ostermann H, Kienast J, Briegel J, Strauss R, Keinecke HO, Warren BL, Opal SM; KyberSept Investigators. High-dose antithrombin III in the treatment of severe sepsis in patients with a high risk of death: efficacy and safety. Crit Care Med. 34: 285-292, 2006.

[28] Warren BL, Eid A, Singer P, Pillay SS, Carl P, Novak I, Chalupa P, Atherstone A, Penzes I, Kubler A, Knaub S, Keinecke HO, Heinrichs H, Schindel F, Juers M, Bone RC, Opal SM, KyberSept Trial Study Group. Caring for the critically ill patient. Highdose antithrombin III in severe sepsis: a randomized controlled trial. JAMA. 286: 1869-1878, 2001.

[29] Pelzer H, Schwarz A, Heimburger N. Determination of human thrombin-AT III complex in plasma with an enzyme-linked immunosorbent assay. Thromb Haemost. 59: 101-107, 1988.

[30] DeMichele MA, Minnear FL. Modulation of vascular endothelial permeability by thrombin. Semin Thromb Hemost. 18(3): 287-295, 1992.

[31] Fleck A, Raines G, Hawker F, Trotter J, Wallace PI, Ledingham IM, Calman KC. Increased vascular permeability: a major cause of hypoalbuminaemia in disease and injury. Lancet. 1(8432): 781-784, 1985.

[32] Fearon KC, Falconer JS, Slater C, McMillan DC, Ross JA, Preston T. Albumin synthesis rates are not decreased in hypoalbuminemic cachectic cancer patients with an ongoing acute-phase protein response. Ann Surg. 227: 249-254.5, 1998.

[33] Schreiber G, Howlett G, Nagashima M, Millership A, Martin H, Urban J, Kotler L The acute phase response of plasma protein synthesis during experimental inflammation. J Biol Chem. 257: 10271-10277, 1982.

[34] Kario K, Matsuo T, Kobayashi H, Matsuo M, Yamamoto K. The transient increase of plasma thrombin-antithrombin III (TAT) complex levels in patients with congenital antithrombin III deficiency during antithrombin III administration is due to extraction of thrombin from fibrin. Thromb Res. 63: 469-472, 1991.

[35] Asakura H, Sano Y, Omote M, Yoshida T, Ontachi Y, Mizutani T, Kaneda M, Yamazaki M, Morishita E, Takami A, Miyamoto K, Nakao S. Decreased plasma activity of 
antithrombin or protein $C$ is not due to consumption coagulopathy in septic patients with disseminated intravascular coagulation. Eur J Haematol. 67: 170-175, 2001.

[36] Fuchs HE, Shifman MA, Pizzo SV. In vivo catabolism of alpha 1-proteinase inhibitortrypsin, antithrombin III -thrombin and alpha 2-macroglobulin-methylamine. Biochim Biophys Acta. 716: 151-157, 1982.

[37] Nakagawa M, Tsuji H, Matsumura H. Changes in post-administered anti-thrombin III activity in congenital AT III deficiency patients. Sinryo to Sinyaku. 22: 2139-2146, 1985. (in Japanese)

[38] Collen D, Schetz J, de Cock F, Holmer E, Verstraete M. Metabolism of antithrombin III (heparin cofactor) in man: effects of venous thrombosis and of heparin administration. Eur J Clin Invest. 7: 27-35, 1977.

[39] Blauhut B, Kramar H, Vinazzer H, Bergmann H. Substitution of antithrombin III in shock and DIC: a randomized study. Thromb Res. 39: 81-89, 1985.

[40] Ilias W, List W, Decruyenaere J, Lignian H, Knaub S, Schindel F, Keinecke HO, Heinrichs $\mathrm{H}$, Thijs LG. Antithrombin III in patients with severe sepsis: a pharmacokinetic study. Intensive Care Med. 26: 704-715, 2000.

[41] Collen D, Schetz J, de Cock F, Holmer E, Verstraete M. Metabolism of antithrombin III (heparin cofactor) in man: effects of venous thrombosis and of heparin administration. Eur J Clin Invest. 7: 27-35, 1977. 

Chapter 3

\title{
Pharmacokinetics of Drugs Following IV Bolus, IV Infusion, and Oral Administration
}

\author{
Tarek A. Ahmed \\ Additional information is available at the end of the chapter \\ http://dx.doi.org/10.5772/61573
}

\section{Introduction}

Pharmacokinetics is the science of the kinetics of drug absorption, distribution, and elimination (i.e., metabolism and excretion). Kinetics is the study of the rate of a process and the factors affecting on it. The rate of a process is the change in velocity or speed with (in relation to) time.

Usually, pharmacokinetics study involves considering both experimental and theoretical approaches. The former involves development of biologic sampling techniques, analytical methods for drugs and their metabolites measurement, and procedures that help in data collection and handling, while the latter approach of pharmacokinetics involves development of pharmacokinetic models that facilitate prediction of drug disposition after drug administration [1].

For the processes of drug absorption, distribution, and elimination, there is a rate that governs each process. The rate is the change in concentration with time and is given by $\pm \mathrm{dC} / \mathrm{dt}$.

According to the law of mass action, the rate of a chemical reaction (or a kinetic process) is proportional to the products of the molar concentration of the reactants each raised to a power equal to the number of molecules of substances undergoing reaction (process).

- The rate expression for zero order reaction:

$$
d C / d t=k_{o} \text { andhence, } C_{t}=C_{o}-k t \text { and } t_{1 / 2}=C_{o} / 2 k
$$

- For first-order reaction:

$$
d C / d t=K_{1} C \& C=C_{o} e^{-k t} \text { and hence, } \log C=\log C_{o}-k t / 2.303 \text { and } t_{1 / 2}=0.693 / k
$$


- For second-order reaction:

$$
\begin{aligned}
& d C / d t=\left[K(A)^{2}\right] \text { or }=[K(A)(B)] \text { or }=\left[K(B)^{2}\right] \\
& \text { andhence, } 1 / C=1 / C_{o}+k t \text { and } t_{1 / 2}=1 / k C_{o}
\end{aligned}
$$

Mostly, the pharmacokinetic processes (absorption, distribution, and elimination) are firstorder, although zero-order also accounts for some processes such as the process of absorption, which is first-order for the passive transport mechanism and zero-order for the carriermediated transport (1).

It must be mentioned that drug kinetics after distribution is characterized by the first-order rate constant.

A compartment in pharmacokinetics is an entity that can be described by a definite volume and a concentration of a drug contained in that volume, which may be:

\section{Central Compartment}

The central compartment includes blood and the highly perfused organs and tissues such as heart, brain, lungs, liver, and kidney. In these organs, the administered drug usually equilibrates rapidly.

\section{Peripheral Compartment(s)}

This compartment(s) include(s) those organs that are less well-perfused such as adipose and skeletal muscle, and therefore the administered drug will equilibrate more slowly in these organs. The duration of the drug effect at the target tissue will often be affected by the redistribution from one compartment to another. For example, the general anesthetic drug, thiopental, which is a highly lipid-soluble agent, induces anesthesia within seconds owing to drug rapid equilibration between blood and brain. The duration of anesthesia is short due to drug redistribution into adipose tissue, which can act as a storage site, or drug reservoir, although thiopental is slowly metabolized.

\section{Special Compartments}

Drug access to some body parts such as the cerebrospinal fluid (CSF) and central nervous system (CNS) is controlled by the structure of the CNS blood capillaries and the outermost layer of the neural tissue, i.e., pericapillary glial cells (the choroid plexus is an exception). Also, some drugs have relatively poor access to pericardial fluid, bronchial secretions, and fluid in the middle ear, thus making the treatment of infections in these regions difficult. These special compartments deserve mention as a separate category.

A pharmacokinetic model is a model devised to simulate the rate process of drug absorption, distribution, metabolism, and elimination with little physiological detail. 
During development and formulation of pharmaceutical dosage forms and drug delivery systems, an interrelationship between these dosage forms/drug delivery systems and biopharmaceutical principals must be established to ensure clinical application of these dosage forms in patient care [2]. Drugs may be introduced into the body via some routes that involve an absorption phase such as oral, topical, intramuscular, subcutaneous, nasal, pulmonary and rectal. For intramuscular, and subcutaneous routes of administration, the absorption is uncomplicated and there is less variability in the absorption process and hence the bioavailability is often considered close to $100 \%$. Drugs that are administered by intravascular route (Intravenous and intrarterial) do not involve an absorption step [3]. Assesment of bioavailability could be achieved from plasma data, urine data, acute clinical response and clinical trials [3].

When a drug is administered into the body, it will distribute into the different organs and body compartments. Analysis of the drug kinetic in the body may be compartmental or noncompartmental. The former is used to describe the drug disposition (distribution and elimination) and the drug concentration in plasma and highly perfused organs assuming that changes in drug plasma concentration are equivalent with that in tissue concentration and the elimination process is achieved from central compartment. The later is used to identify certain pharmacokinetic parameters without deciding on a particular compartmental model. The basic calculations are based on the area under the plasma concentration versus times curve (zero moment) and the first moment curve (AUMC).

\section{One-Compartment Open Model: Intravenous Bolus Administration}

This model represents the simplest way to describe the process of drug distribution as well as elimination in the body. In this model, the body acts like a single, uniform unit in which the drug can enter or leave the body easily (i.e., the model is "open" for the drug movement).

For IV bolus administered drugs, the entire dose enters the bloodstream directly. This is followed by distribution of the drug through the circulatory system to all the tissues in the body. Concentration of the drug in various tissue organs or the process of drug distribution in the body will occur depending on the blood flow to the tissue, the molecular weight of the drug, the drug lipophilicity, plasma protein binding, and the binding affinity of the drug toward certain tissue.

Mostly, drugs are eliminated from the body either through the kidney and/or the liver following drug metabolism.

The first pharmacokinetic parameter that arises in this model is the apparent volume of distribution, $V_{\mathrm{D}}$, which is the volume in which the drug is distributed on it within the body, while the elimination rate constant, $k$, is the second pharmacokinetic parameter in this model, which governs the rate at which the drug concentration in the body declines over time.

A representative diagram that describes this model is illustrated below: 
IV dose $\mathrm{DB}, \mathrm{VD}$ elimination

This model does not give information about the actual drug levels in the body tissues. However, the model assumes that any changes in the plasma drug levels will always result in a relative change in tissue drug levels.

The general equation that describes this model is:

$$
\begin{gathered}
d C_{p} / d t=-k C p \text { and } C p=C p^{o} \cdot e^{k e l t} \text { and } \log C p=\log C p^{o}-k_{e l} \cdot t / 2.303 \\
\text { Or } d D_{B} / d t=-k D_{B} \text { and } D B=D_{B}{ }^{o} \cdot e^{k e l t} \text { So, } \log D_{B}=\log D_{B}^{o}-k_{e l} \cdot t / 2.303
\end{gathered}
$$

\subsection{Elimination Rate Constant}

For most drugs, the process of drug elimination is a first-order rate process, i.e., the process is dependent on the amount or concentration of drug present, and the unit of the elimination rate constant $k$ is time $\mathrm{t}^{-1}$ (e.g., $\mathrm{hr}^{-1}$ or $1 / \mathrm{hr}$ ).

Total removal or elimination of the parent drug from this compartment is effected by metabolism (biotransformation) and excretion. So, this constant represents the sum of these two processes:

$$
k=k m+k e
$$

A rate expression for the first-order elimination is:

$$
d D_{B} / d t=-k D_{B}
$$

Integration of the above equation gives the following expression:

$$
D_{B}=D_{B}^{0} e^{k t}
$$

The last equation can also be expressed in the logarithmic form as:

$$
\log D_{B}=\log D_{B}^{0}-k t / 2.303
$$




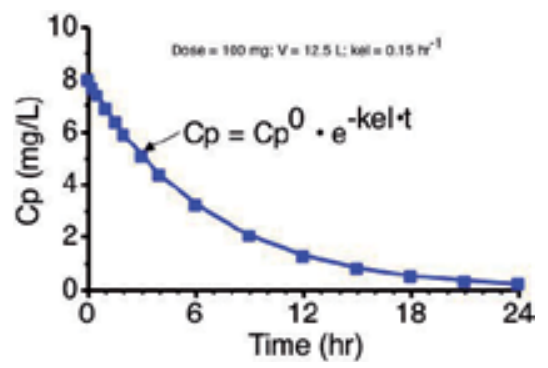

Figure 1. Linear Plot of $\mathrm{Cp}$ versus Time for a One-Compartment IV Bolus

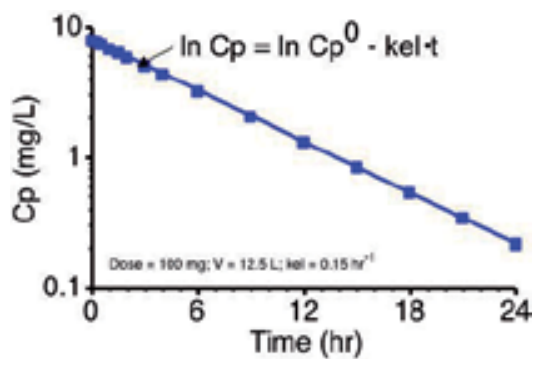

Figure 2. Semilog Plot of $\mathrm{Cp}$ versus Time

\subsection{Apparent Volume of Distribution}

The volume that must be considered in estimating the amount of drug in the body from the concentration of drug found in the sampling compartment is referred to as volume of distribution. This volume does not represent a physical space in the body but it is a dilution space. In other words, it is a theoretical volume that represents the distribution of the administered drug between the plasma and the rest of the body following administration of the medicament.

To predict the volume of distribution we must assume that the amount of the drug in the body $\left(D_{B}\right)$ is the function of both the concentration of the drug in the plasma $\left(C_{P}\right)$ and the volume of fluid the drug is distributed in.

Since; $D_{B}=V_{D} C_{P}$

Assuming $D_{B}$ is the IV dose

$$
V D=I V \text { dose }\left(D_{B}\right) / C p
$$

The amount of drug in the body is estimated by taking a blood sample at periodic intervals and analyzing for the concentration of drug present. So, it is not determined directly. 
Similar expression based on drug concentration in plasma is obtained for the first-order decline of drug plasma levels:

$$
\log C_{P}=\log C_{P}-k t / 2.303
$$

\subsubsection{Calculation of Volume of Distribution}

This pharmacokinetic parameter, volume of distribution $\left(V_{\mathrm{D}}\right)$, is calculated in the onecompartment model (IV administration) using the following equation:

$$
V_{D}=I V \text { dose } / C_{P}^{0}=D_{B}^{0} / C_{P}^{0}
$$

Since the rate of drug elimination is: $d D_{B} / d t=-k D_{B}$

substitution of $D_{B}=V_{D} C_{P}$; so, $d D_{B} / d t=-k V_{D} C_{P}$

Rearrangement of the equation: $d D_{B}=-k V_{D} C_{P} d t$

Integration of the last equation and assuming AUC is the summation of the area under the curve from $\mathrm{t}=0$ to $\mathrm{t}=\alpha$ :

$$
D_{0}=k V_{D}[A U C]_{0}^{\alpha}
$$

Thus, the apparent $V_{D}$ may also be obtained from knowledge of the dose, elimination rate constant, and the area under the curve (AUC) from $t=0$ to $t=\infty$ :

$$
V_{D}=D_{0} / k[A U C]_{0}^{\alpha}
$$

N.B: The $\mathrm{AUC}^{\infty}{ }_{0}$ is usually estimated by the trapezoidal rule.

\subsubsection{Significance of the Apparent Volume of Distribution}

Assuming the following equation:

$$
V_{D}=I V \text { dose } / C_{P}^{0}=D_{B}^{0} / C_{P}^{0}
$$

The apparent $V_{\mathrm{D}}$ is dependent on $C_{\mathrm{p}}{ }^{0}$. Drugs characterized by a large apparent $V_{\mathrm{D}}$ are mainly concentrated in extravascular tissues and are less concentrated in the intravascular capillaries. In case of drugs that are highly bound to plasma proteins or remain in the vascular capillaries, as for highly hydrophilic drugs, the $C_{\mathrm{p}}{ }^{0}$ will be high and this is resulting in a smaller $V_{\mathrm{D}}$. 
It must be mentioned that the apparent volume of distribution is not a true physiologic volume. This pharmacokinetic parameter is useful in considering distribution and the relative amounts of drug in both vascular and extravascular tissues.

\subsection{Clearance}

Clearance or drug clearance is a pharmacokinetic term describing the process of drug elimination from the body without identifying the mechanism of the process. It refers to the volume of plasma fluid that is cleared of drug per unit time (volume approach, $\mathrm{L} / \mathrm{hr}$ or $\mathrm{mL} / \mathrm{hr}$ ) or the amount of the drug eliminated from the body per unit time (mass approach, $\mathrm{mg} / \mathrm{min}$ or $\mathrm{mg}$ / hr). It may also be considered as the fraction of the drug $V_{\mathrm{D}}$ that is excreted by the kidney per unit time (fraction approach). It must be mentioned that a constant volume of plasma (about $120 \mathrm{~mL} / \mathrm{min}$ in humans) is filtered through the glomeruli of the kidneys. For drugs that exhibit significant plasma protein binding, clearance is related to the total drug concentration in the plasma (free + protein-bound) and not the free concentration [5].

For drugs that are eliminated by first-order elimination process, the elimination rate is not constant and changes with respect to the drug concentration in the body, and hence, the drug clearance is expressed as volume per unit time (e.g., $\mathrm{L} / \mathrm{hr}$ or $\mathrm{mL} / \mathrm{min}$ ). This is convenient because the volume per unit time is a constant, whereas for drugs that are eliminated from the body by a zero-order elimination process, expressing the rate of drug elimination as mass per unit time is convenient because the clearance rate is constant and does not depend on the drug plasma concentration.

Mathematically, since the rate of drug change (elimination) in the body is dependent on the drug plasma concentration $\mathrm{C}_{\mathrm{P}}$,

$d D_{B} / d t=-k D_{B}$ and since $D_{B}=V_{D} C_{P}$

so, $d D_{B} / d t=-k V_{D} C_{P}$

Dividing the above equation by $\mathrm{C}_{\mathrm{P}}$

$\left[d D_{B} / d t\right] / C_{P}=-\left[k V_{D} C_{P}\right] / C_{P}$; then, $\left[d D_{B} / d t\right] / C_{P}=-k V_{D}=-$ clearance

The term $\left[\mathrm{dD}_{\mathrm{B}} / \mathrm{dt}\right] / \mathrm{C}_{\mathrm{P}}$ in the above equation is considered clearance according to the fraction approach.

The term fraction of total drug eliminated is applicable and convenient during expressing drug elimination whether one is dealing with an amount or a volume because of its dimensionless nature.

\subsection{Calculation of ' $k$ ' from Urinary Excretion Data}

Assuming the excretion rate of the drug is first-order, the elimination rate constant $k$ may be calculated from urinary excretion data. The term $k_{\mathrm{e}}$ is the renal excretion rate constant, and $D_{\mathrm{u}}$ is the amount of drug excreted in the urine.

$d D_{u} / d t=k_{e} D_{B}, D_{\text {B }}$ can be substituted for $D_{B} 0 e^{-k t}$ 
So, $d D_{u} / d t=k_{e} D_{B}^{0} e^{-k t}$

and $\log d D_{u} / d t=\log k_{e} D_{B}^{0}-k t / 2.303$

Plotting $\log d D_{\mathrm{u}} / d t$ versus time on regular paper or on semilog paper $d D_{\mathrm{u}} / d t$ against time results in a straight line in which the slope of this curve is equal to $-k / 2.3$ and the $y$ intercept is equal to $k_{\mathrm{e}} D_{\mathrm{B}}{ }^{0}$. If $D_{\mathrm{B}}{ }^{0}$ is known, the renal excretion rate constant $\left(k_{\mathrm{e}}\right)$ can be obtained.

For nonrenal rate constant $\left(\mathrm{k}_{\mathrm{nr}}\right)$, i.e., for any route of elimination other than renal excretion, it could be estimated from: $\mathrm{k}-\mathrm{k}_{\mathrm{e}}=\mathrm{k}_{\mathrm{nr}}$

N.B: Experimentally, it is difficult to determine the drug urinary excretion rate $\left(\mathrm{dD}_{\mathrm{u}} / \mathrm{dt}\right)$ and it is more convenient to plot the average rate of urinary drug excretion $\left(\mathrm{Du} / \mathrm{t}_{\text {interval }}\right)$ against the average time $t^{*}$ for the collected urine samples. $\mathrm{Du} / \mathrm{t}$ is the amount of drug excreted divided by the time interval.

\section{Practice Example}

A single intravenous dose of an antibiotic was administered to a 50-kg woman at a dose level of $20 \mathrm{mg} / \mathrm{kg}$. Samples of urine and blood were removed periodically and assayed for parent drug. The following data were obtained:

\begin{tabular}{ccc}
\hline Time (hr) & Cp $(\mu \mathrm{g} / \mathbf{m l})$ & Du (mg) \\
\hline 0.25 & 4.2 & 160 \\
\hline 0.50 & 3.5 & 140 \\
\hline 1.0 & 2.5 & 200 \\
\hline 2.0 & 1.25 & 250 \\
\hline 4.0 & 0.31 & 188 \\
\hline 6.0 & 0.08 & 46 \\
\hline
\end{tabular}

\section{Solution}

Here $t^{*}=$ midpoint of collection period; and $t=$ time interval for collection of urine sample.

To solve this problem, first plot on a semilogarithmic scale the relation between $D_{\mathrm{u}} / t$ versus $t^{*}$. A straight line is obtained; the slope of this line should equal $-k / 2.3$. Then, determine the elimination $\mathrm{t}_{1 / 2}$ directly from the curve and calculate $k$ from the first-order equation $\mathrm{k}=0.693 / \mathrm{t}_{1 / 2}$

\begin{tabular}{ccccc}
\hline Time $(\mathbf{h r})$ & $\left.\mathbf{D}_{\mathbf{u}} \mathbf{( m g}\right)$ & Time Interval & $\left.\mathbf{D}_{\mathbf{u}} / \mathbf{t} \mathbf{( m g} / \mathbf{h r}\right)$ & $\mathbf{t}^{*}$ \\
\hline 0.25 & 160 & $0-0.25=0.25$ & $160 / 0.25=640$ & $(0+0.25) / 2=0.125$ \\
\hline 0.50 & 140 & $0.5-0.25=0.25$ & $140 / 0.25=560$ & $(0.25+0.5) / 2=0.375$ \\
\hline 1.0 & 200 & $1-0.5=0.5$ & $200 / 0.5=400$ & $(0.5+1) / 2=0.750$ \\
\hline 2.0 & 250 & $2-1=1$ & $250 / 1=250$ & $(1+2) / 2=1.5$ \\
\hline 4.0 & 188 & $4-2=2$ & $188 / 2=94$ & $(2+4) / 2=3$ \\
\hline 6.0 & 46 & $6-4=2$ & $46 / 2=23$ & $(4+6) / 2=5$ \\
\hline
\end{tabular}

In this example, $\mathrm{t}_{1 / 2}=1.0 \mathrm{hr}$ and $k=0.693 \mathrm{hr}^{-1}$. 
The sigma-minus method, or the amount of drug remaining to be excreted method, is an alternative method for calculation of the elimination rate constant $k$ from urinary excretion data is. This method is sometimes preferred over the previous method since minimum fluctuations in the rate of elimination is obtained by this method.

If the amount of unchanged drug that is ultimately excreted in the urine is $D^{\infty}{ }_{\mathrm{u}}$ and, $D_{\mathrm{u}}$ is the cumulative amount of unchanged drug excreted in the urine at a specific time. The amount of the drug remaining to be excreted is $\mathrm{D}^{\infty}{ }_{\mathrm{u}}-D_{\mathrm{u}}$ in which the rate of excretion of this amount is dependent on or proportional to $D^{\infty}{ }_{u}$ as the process is a first-order and so,

$$
D_{u}^{\infty}-D_{u}=D^{\infty}{ }_{u} \cdot e^{-k t}
$$

The last equation can be written in the logarithmic form as:

$$
\log \left(D_{u}^{\infty}-D_{u}\right)=\log D_{u}^{\infty}-k t / 2.303
$$

This equation describes the relationship for the amount of drug remaining to be excreted $\left(D^{\infty}{ }_{\mathrm{u}}-D_{\mathrm{u}}\right)$ versus time.

A linear curve is obtained by plotting on a semilog paper the amount of the drug unchanged yet to be eliminated, $\log \left(D^{\infty}{ }_{u}-D_{u}\right)$ versus time, the slope of this curve is $-k / 2.3$, and the $y$ intercept is $D^{\infty}{ }_{\mathrm{u}}$.

\section{Practice Example}

Using the data in the preceding problem, determine the elimination rate constant.

\begin{tabular}{ccc}
\hline Time (hr) & Cp $(\mu \mathrm{g} / \mathbf{m l})$ & $\mathbf{D}_{\mathbf{u}}(\mathbf{m g})$ \\
\hline 0.25 & 4.2 & 160 \\
\hline 0.50 & 3.5 & 140 \\
\hline 1.0 & 2.5 & 200 \\
\hline 2.0 & 1.25 & 250 \\
\hline 4.0 & 0.31 & 188 \\
\hline 6.0 & 0.08 & 46 \\
\hline
\end{tabular}

\section{Solution}

\begin{tabular}{cccc}
\hline Time (hr) & $\mathbf{D}_{\mathbf{u}}(\mathbf{m g})$ & Cumulative $\mathbf{D u}$ & $\mathbf{D}^{\infty}{ }_{\mathbf{u}}-\mathbf{D}_{\mathbf{u}}$ \\
\hline 0.25 & 160 & 160 & $984-160=824$ \\
\hline 0.50 & 140 & $160+140=300$ & $984-300=684$ \\
\hline 1.0 & 200 & $300+200=500$ & $984-500=484$ \\
\hline 2.0 & 250 & $500+250=750$ & $984-750=234$ \\
\hline 4.0 & 188 & $750+188=938$ & $984-938=46$ \\
\hline 6.0 & 46 & $938+46=984$ & $984-984=0$ \\
\hline
\end{tabular}


Plot $\log \left(D_{\mathrm{u}}^{\infty}-D_{\mathrm{u}}\right)$ versus time. Use a semilogarithmic scale for $\left(D_{\mathrm{u}}^{\infty}-D_{\mathrm{u}}\right)$. Evaluate $k$ and $\mathrm{t}_{1 / 2}$ from the slope.

\section{Multicompartment Models: Intravenous Bolus Administration}

\subsection{Introduction}

It was observed that the plasma-level time curve for some drugs following its rapid IV injection does not decline linearly as a single, first-order rate process. This nonlinear plasma-level time curve is attributed to distribution of these drugs at various rates into different tissue groups. So, these multicompartment models were developed to explain and also to predict the plasma and tissue concentrations of these drugs. Again, drug distribution in the body depends mainly on plasma protein binding, tissue affinity, and drug lipo- or hydrophilicity.

It must be mentioned that the kinetic description of the multicompartment process assumes that the rate of drug transfer between central and tissue compartments is first-order.

It is noteworthy to mention that the body can be divided into organs with high blood perfusion and those with slow blood perfusion. The heart, brain, liver, lungs, kidney, endocrine glands, skin and muscle, adipose tissue, and marrow, which account for $78 \%$ of the body weight, are highly blood-perfused organs, while bones, ligaments, tendons, cartilage, teeth and hair which comprise the remaining $22 \%$ of the body weight are slowly blood-perfused.

The central compartment consists of the plasma, extracellular fluids and highly perfused tissues in which drug equilibrate rapidly. The kidney and liver, which are the tissues for drug elimination, are considered integral parts of the central compartment.

\subsection{Two-Compartment Open Model}

The plasma-level time curve for a drug that follows a two-compartment model shows that the plasma drug concentration declines biexponentially as the sum of two first-order processesdistribution and elimination. This is attributed to distribution of the administered drug between the central compartment and tissue (peripheral compartment).

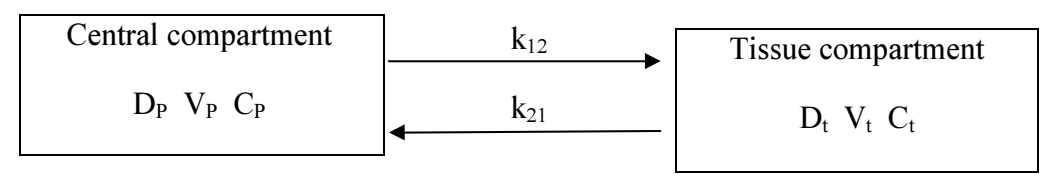

Figure 3. Figure shows distribution of the administered drug between central and peripheral compartment

Construction of the plasma-level time curve for a drug that follows a two-compartment model indicates that the curve may be divided into two phases: a distribution phase and an elimination phase. At $t=0$, no drug is in the tissue compartment and hence, the distribution phase of 
the curve begins and represents the initial, more rapid decline of drug from the central compartment into the tissue compartment. Later, the drug starts to enter the tissue (peripheral compartment) and when the drug reaches the maximum tissue concentrations, an equilibrium is established and the rate of drug entry into the tissue equals the rate of drug exit from the tissue. At this stage, the drug concentrations in the central and tissue compartments will decline in a parallel and slower manner when compared to the distribution phase. This phase is the elimination phase and the decline is a first-order process.

For most two-compartment models the elimination occurs from the central compartment model unless other information about the drug is known since the major sites of drug elimination (renal excretion and hepatic metabolism) occur from organs such as the kidney and liver, which are highly perfused with blood $[6,7]$.

If $k_{12}$ and $k_{21}$ are first-order rate constants that govern the rate of drug change in and out of the tissues, then the change in drug concentration in the tissue with time could be calculated from the following equation:

$$
d C_{t} / d_{t}=k_{12} C_{P}-k_{21} C_{t}
$$

And since $C_{P}=D_{P} / V_{P}$ and $C_{t}=D_{t} / V_{t}$

then $d C_{t} / d_{t}=k_{12} D_{P} / V_{P}-k_{21} D_{t} / V_{t}$

where $D_{\mathrm{p}}$ represents the amount of drug in the central compartment, $D_{\mathrm{t}}$ is the amount of drug in the tissue compartment, $V_{\mathrm{p}}$ represents the volume of drug in the central compartment, and $V_{\mathrm{t}}$ is the volume of drug in the tissue compartment.

The mathematical expression that best describes the two-compartment IV bolus is:

$$
C_{P}=A \cdot e^{-a t}+B \cdot e^{-b t}
$$

The constants $a$ and $b$ represent the rate constants for the two phases, distribution phase and elimination phase, respectively. The constants $A$ and $B$ are intercepts on the $y$ axis obtained from the plasma-level time curve after IV bolus, which exhibit two compartments. These values may be obtained graphically by the method of residuals or by computer.

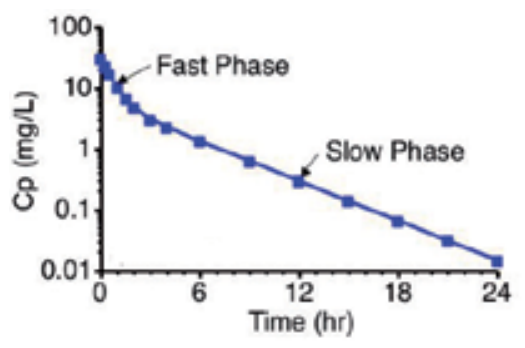

Figure 4. Semilog plot of plasma-level versus time for a two-compartment IV bolus model 


\subsubsection{Method of Residuals}

This method is used for fitting curve into the experimental data when the drug does not follow a one-compartment model. The method is sometimes called Feathering or Peeling method.

\section{Practice Example}

A 70-kg patient was administered a drug by rapid IV injection in a dose of $100 \mathrm{mg}$. Blood samples were taken periodically after the administration of drug, and the plasma samples were assayed for the drug concentration. The following data were obtained:

\begin{tabular}{cc}
\hline Time (hr) & Plasma Concentration $(\mu \mathrm{g} / \mathrm{ml})$ \\
\hline 0.25 & 43 \\
\hline 0.5 & 32 \\
\hline 1 & 20 \\
\hline 1.5 & 14 \\
\hline 2 & 11 \\
\hline 4 & 6.5 \\
\hline 8 & 2.8 \\
\hline 12 & 1.2 \\
\hline 16 & 0.52 \\
\hline
\end{tabular}

If you plotted the provided data on semilogarithmic graph paper, a curved line is observed which indicates that the drug is distributed in more than one compartment.

From the data, the constants may be obtained either by the computer or by the method of residuals, in which the equation that describes the process is:

$$
C_{P}=A e^{-a t}+B e^{-b t}
$$

Plotting of the data indicates that the curve is biexponential: the first segment for the distribution phase (rapid phase), while the second for the elimination phase. The rapid distribution phase is confirmed when comparing the values for $a$ and $b$, the constant $a$ being larger than the rate constant $b$. Therefore, at some later time, the term $A e^{\text {-at }}$ will approach zero, while $B e^{-}$ ${ }^{b t}$ will still have a value. At this later time, the two-compartment IV bolus equation will reduce to:

$$
\begin{aligned}
C_{P} & =B e^{-b t} \\
C_{P}^{\text {latw }} & =B \cdot e^{-\beta \cdot t}
\end{aligned}
$$

And the logarithmic form is: 


$$
\log C_{P}=\log B-b t / 2.303 .
$$

It is possible to calculate the rate constant $b$ from the slope $(-b / 2.3)$ of a straight line representing the terminal exponential phase in which $b$ was found to be $0.21 \mathrm{hr}^{-1}$. The $t_{1 / 2}$ for the elimination phase (beta half-life) can be derived from:

$$
t_{1 / 2}=0.693 / b
$$

The regression line for the terminal phase could be extrapolated to the $y$ axis, in which the $y$ intercept is equal to $B$, or $15 \mu \mathrm{g} / \mathrm{mL}$.

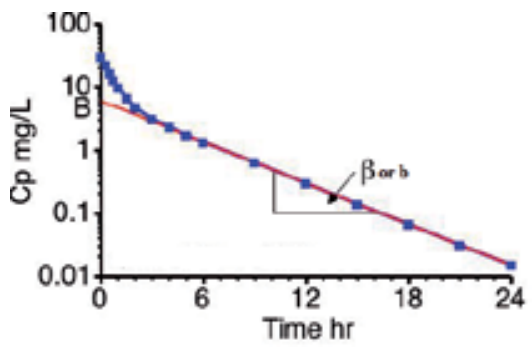

Figure 5. Semilog Plot of $\mathrm{Cp}$ versus Time Showing $C_{p}^{\text {late }}$

To obtain the constant a, the values for the drug plasma concentrations at the extrapolated line are subtracted from the original experimental data point to get the residual plasma concentration. Plotting the values of the residual plasma concentrations versus time will yield a straight line that represents the rapid distribution (a) phase. The slope of the line (-a /2.3) and

\begin{tabular}{|c|c|c|c|}
\hline Time (hr) & $\begin{array}{l}\text { Plasma Concentration } \\
\qquad(\mu \mathrm{g} / \mathrm{ml})\end{array}$ & $\begin{array}{l}\text { Extrapolated Plasma } \\
\text { Concentration } C_{P}^{\prime}\end{array}$ & $\begin{array}{c}\text { Residual Plasma } \\
\text { Concentration } C_{P}-C_{P}^{\prime}\end{array}$ \\
\hline 0.25 & 43 & 14.5 & 28.5 \\
\hline 0.5 & 32 & 13.5 & 18.5 \\
\hline 1 & 20 & 12.3 & 7.7 \\
\hline 1.5 & 14 & 11 & 3 \\
\hline 2 & 11 & 10 & 1 \\
\hline 4 & 6.5 & & \\
\hline 8 & 2.8 & & \\
\hline 12 & 1.2 & & \\
\hline 16 & 0.52 & & \\
\hline
\end{tabular}
the value of (a) is $1.8 \mathrm{hr}^{-1}$, and the y intercept is $45 \mu \mathrm{g} / \mathrm{mL}$. 


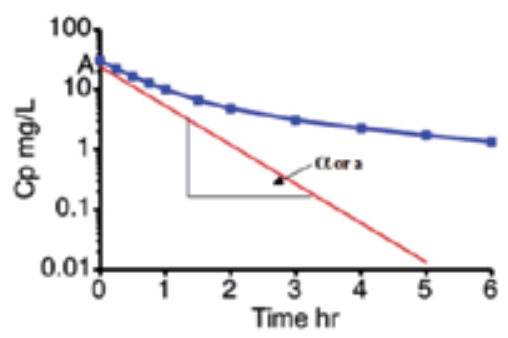

Figure 6. Semilogplot of $\mathrm{Cp}$ versus time showing residual line

A number of pharmacokinetic parameters may be derived by proper substitution of rate constants $a, b$, and $y$ intercepts $(A$ and $B)$ into the following equations:

$$
\begin{gathered}
k=a b(A+B) / A b+B a, \quad k_{12}=A B(b-a)^{2} /(A+B)(A b+B a) \\
k_{21}=A b+B a / A+B
\end{gathered}
$$

\subsubsection{Apparent Volumes of Distribution}

As mentioned previously, drugs with extravascular distribution such as those with high peripheral tissue binding contribute to a large apparent volume of distribution, while drugs that are polar with low lipid solubility or which highly bound to plasma protein account for small apparent $\mathrm{V}_{\mathrm{D}}$.

In multiple-compartment kinetics, such as the two-compartment model, several volumes of distribution can be calculated.

\subsubsection{Volume of the Central Compartment (At Zero Time)}

It is important to mention that the adult person has around $5 \mathrm{~L}$ blood; $45 \%$ of this volume is cellular part and $55 \%$ noncellular part, of which $90 \%$ of the noncellular part is plasma, and so the volume of plasma in the central compartment is approximately greater than $3 \mathrm{~L}$.

For many polar drugs, an initial volume of 7-10 L may be interpreted as rapid distribution of the administered drug within the plasma and some body extracellular fluids. For example, the $V_{\mathrm{p}}$ of the antibiotic moxalactam ranges from 0.12 to $0.15 \mathrm{~L} / \mathrm{kg}$, corresponding to about 8.4 to 10.5 L for a typical 70-kg patient.

In the two-compartment model, if we multiply the drug concentration by the volume of the fluid, it must equal to the dose at time zero since $D_{0}=V_{\mathrm{p}} C_{\mathrm{p}}$. If this is true, then the volume of distribution will equal $3 \mathrm{~L}$ and if not, then distribution of drug may also occur outside the vascular pool.

The apparent volume of the central compartment is: 


$$
V_{p}=D_{o} / C p^{o}
$$

$\mathrm{D}_{\mathrm{o}}$ is the entire amount of the drug in the body which is equivalent to the IV dose while, $C \mathrm{p}^{\circ}$ is the initial concentration of the drug in the central compartment or the concentration at time = zero.

At zero time $(\mathrm{t}=0)$, the total drug in the body is in the central compartment. $C_{\mathrm{p}}^{0}$ will equal to $A+B$ by the following equation:

$$
C p=A \cdot e^{-a t}+B \cdot e^{-b t}
$$

At $\mathrm{t}=0, \mathrm{e}^{0}=1$ and so, $C p=A+B$

And so, $V p=D_{o} / A+B$

Instead, the volume of the central compartment $\mathrm{Vp}$ may be calculated from the area under the curve $[\mathrm{AUC}]^{\alpha}{ }_{0}$ in a manner similar to the calculation of the apparent $V_{\mathrm{D}}$ in the one-compartment model.

$$
[A U C]_{0}^{\alpha}=D_{o} / k V_{D}
$$

In contrast, $[\mathrm{AUC}]^{\alpha}{ }_{0}$ for the two-compartment model is Do / $\mathrm{k} \mathrm{V}_{\mathrm{P}}$

And so,

$$
V p=D_{o} / k[A U C]_{0}^{\alpha}
$$

\subsubsection{Apparent Volume of Distribution at Steady-State}

When the rate of drug entering the peripheral (tissue) compartment from the central compartment is equal to the rate of drug exit from the tissue compartment into the central compartment, this condition is achieved at steady-state and the rates of drug transfer between the two compartments are described by the following expressions:

$$
\begin{gathered}
D_{t} k_{21}=D_{p} k_{12} \\
D_{t}=k_{12} D_{p} / k_{21}
\end{gathered}
$$

Since the amount of drug in the central compartment, $D_{p}$, is equal to $V_{p} C_{p}$, so, $D_{t}=k_{12} V_{p} C_{p} / k_{21}$

Assuming a steady-state condition is reached, therefore the apparent volume of drug distribution at steady-state $\left(V_{\mathrm{D}}\right)_{\mathrm{ss}}$ may be determined by dividing the total amount of drug in the body by the concentration of drug in the central compartment at steady-state: 


$$
\begin{gathered}
\left(V_{D}\right)_{S S}=D_{p}+D_{t} / C_{p} \\
\left(V_{D}\right)_{S S}=\left[V_{p} C_{p}+k_{12} V_{p} C_{p} / k_{21}\right] / C_{p} \\
\left(V_{D}\right)_{S S}=V_{p}+k_{12} / k_{21} V_{p}
\end{gathered}
$$

\subsubsection{Volume of Distribution by Area}

The volume of distribution by area, also known as $\left(V_{\mathrm{D}}\right)_{\text {arear }}$ or simply $\left(V_{\mathrm{D}}\right)$, is obtained by a method similar to that used to find $V_{\mathrm{p}}$ except that the rate constant $b$ is used instead of the overall elimination rate constant $k$. This is achieved through dividing the total body clearance by $b$ and is influenced by drug elimination in the beta, or $b$, phase.

So, the reduction in drug clearance from the body may increase the area under the curve AUC, which is reflected on the value of $\left(V_{\mathrm{D}}\right)$ that is either reduced or unchanged depending on the value of $b$.

$$
\left(V_{D}\right)_{\beta}=\left(V_{D}\right)_{\text {area }}=D_{o} / b \cdot[A U C]_{0}^{\alpha}
$$

Since total body clearance is equal to $D_{o} /[A U C]_{0}^{\infty}$,

so, $\left(V_{D}\right)_{\beta}=$ clearance $/ b=k V p / b$

Here the volume of distribution is related to the body clearance, and the body clearance usually occurs during the elimination phase.

\section{Example}

The first two columns of the provided table represent the time and plasma concentration, which may be collected after IV bolus administration of $500 \mathrm{mg}$ of drug.

\begin{tabular}{cccc}
\hline Time (hr) & $\begin{array}{c}\text { Plasma Concentration } \\
(\mathbf{m g} / \mathbf{m l})\end{array}$ & $\begin{array}{c}\text { Extrapolated Plasma } \\
\text { Concentration } \\
\mathbf{C}^{\prime} \mathbf{P}\end{array}$ & $\begin{array}{c}\text { Residual Plasma Concentration } \\
\mathbf{C P}-\mathbf{C}^{\prime} \mathbf{P}\end{array}$ \\
\hline 0.25 & 20.6 & 8.8 & 11.8 \\
\hline 1 & 13.4 & 7.8 & 5.6 \\
\hline 2 & 7.3 & 6.1 & 1.2 \\
\hline 3 & 5 & 4.7 & - \\
\hline 4 & 3.7 & 3.7 & \\
\hline 6 & 2.2 & & \\
\hline 8 & 1.4 & & \\
\hline 10 & 0.82 & & \\
\hline 12 & 0.5 & & \\
\hline
\end{tabular}


If these data are plotted, the following figure 7 is obtained:

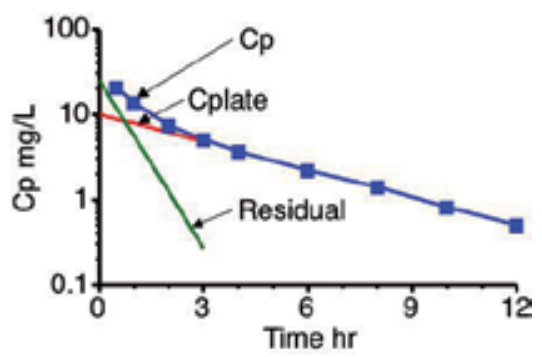

Figure 7. Plot of $\mathrm{Cp}$ versus Time Illustrating the Method of Residuals

At $t=0$ gives $B=10 \mathrm{mg} / \mathrm{L}$. From the slope of the line $\beta=0.25 \mathrm{hr}^{-1}$. C extrapolated values at early times are shown in column 3 and the residual in column 4 . The residual values are plotted again, giving a value of $\mathrm{A}=25 \mathrm{mg} / \mathrm{L}$ and $\alpha=1.51 \mathrm{hr}^{-1}$. Note that $\alpha / \beta=6$; thus, these values should be fairly accurate.

$$
\begin{aligned}
& B=10 \mathrm{mg} / \mathrm{L}, \quad \beta=(\ln 10-\ln 0.5) / 12=2.996 / 12=0.25 h r^{-1} \\
& A=25 \mathrm{mg} / \mathrm{L}, \quad \alpha=(\ln 25-\ln 0.27) / 3=4.528 / 3=1.51 h r^{-1}
\end{aligned}
$$

Therefore $C p=25 \cdot e^{-1.51 \cdot t}+10 \cdot e^{-0.25 \cdot t}$

$$
\begin{gathered}
k_{21}=\frac{A \cdot \beta+B \cdot \alpha}{A+B}=\frac{25 \times 0.25+10 \times 1.51}{25+10}=0.61 \mathrm{hr}^{-1} \\
k_{e l}=\frac{\alpha \cdot \beta}{k_{21}}=\frac{1.51 \times 0.25}{0.61}=0.62 \mathrm{hr}^{-1} \\
k_{12}=\alpha+\beta-k_{21}-k_{e l}=1.51+0.25-0.61-0.62=0.53 \mathrm{hr}^{-1} \\
V_{1}=\frac{\text { Dose }}{A+B}=\frac{500}{35}=14.3 \mathrm{~L} \\
V_{\text {area }}=\frac{\text { Dose }}{\beta \cdot A U C}=\frac{500}{0.25 \times 58.3}=34.3 \mathrm{~L}
\end{gathered}
$$




$$
V_{s s}=V_{1} \frac{k_{21}+k_{12}}{k_{21}}=14.3 \times \frac{0.61+0.62}{0.61}=26.7 \mathrm{~L}
$$

\subsubsection{Drug Clearance}

The concept for drug clearance that follows a two-compartment model is similar to that of the one-compartment. Clearance may be calculated without consideration of the compartment model. Clearance is calculated by dividing the IV bolus dose by the area under the plasmalevel time curve from zero to infinity.

$$
C_{l}=D_{o} /[A U C]_{o}^{\alpha}
$$

The last equation could also be expressed as a function of volume of distribution:

Since $\left(V_{D}\right)_{\beta}=D_{o} / b \cdot[A U C]_{0}^{\alpha}$

So, $C_{l}=\left(V_{D}\right)_{\beta} b$

The last equation is simple and gives more accurate results than using the trapezoidal rule to obtain area.

\section{Intravenous infusion}

\subsection{Introduction}

Drugs administered by IV route may either be given at once (as a bolus dose) or by slower IV infusion over a definite time such as Phenytoin which must be given slowly, no greater than $50 \mathrm{mg} / \mathrm{min}$ (and preferably $25 \mathrm{mg} / \mathrm{min}$ or less) in adults. Such drugs are infused slowly through a vein into the blood at a constant rate (zero order input) which allows precise control of plasma drug concentrations.

The following figure represents the plasma-level time curve for a drug given by constant IV infusion. At time zero, no drug was present in the body after which the drug level gradually increases until it becomes constant (plateau or steady-state). Once the drug has reached the steady-state, the rate of drug leaving the body is equal to the rate of drug entering the body.

The mathematical expression or the pharmacokinetic equation for drug administered by infusion will depend on whether the drug follows the one- or two-compartment model.

\subsection{One-Compartment IV Infusion}

Drugs administered by constant IV infusion show a zero-order input process, during which the drug is introduced into the bloodstream while the elimination process for most drugs is 


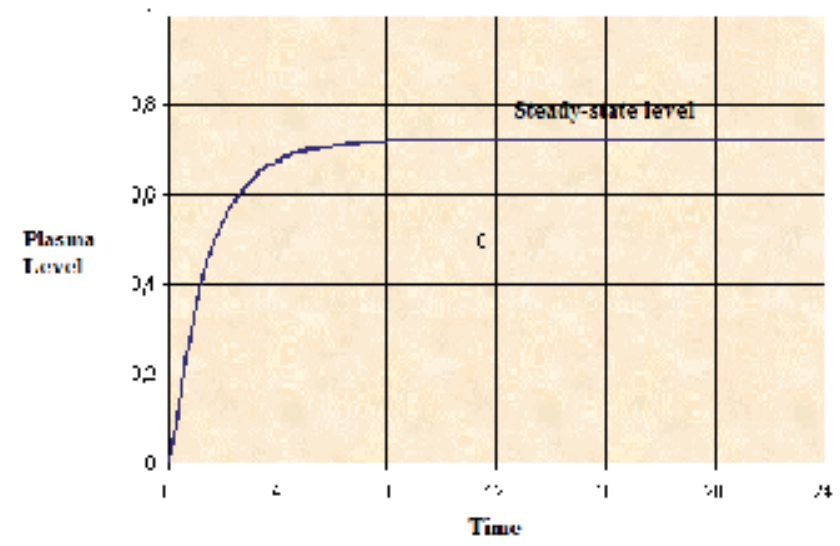

Figure 8. Plasma-concentration time curve during the infusion of the administereddrug at constant rate

first-order. The rate of input minus the rate of output represents the change in the amount of drug in the body at any given time $\left(d D_{\mathrm{B}} / d t\right)[1]$.

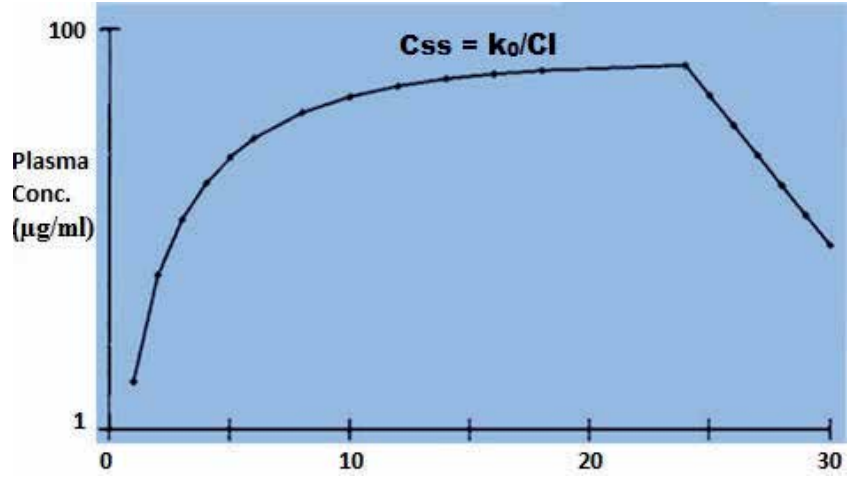

Figure 9. Plasma-concentration time curve after IV Infusion

If $D_{B}$ is the amount of the drug in the body, $R$ is the rate of drug input (infusion rate) and $k$ is the elimination rate constant. The expression that best describes the process is:

$$
\begin{gathered}
d D_{B} / d t=R-k D_{B} \\
d D_{B} / d t=R-k C_{P} V_{D}
\end{gathered}
$$

Integration of the last equation will give: 


$$
C_{P}=\left[R / V_{D} k\right]\left(1-e^{-k t}\right)
$$

At infinite time, $\mathrm{t}=\alpha$ and $\mathrm{e}^{-\mathrm{kt}}$ will approach zero (at steady-state) and the last equation will be:

$$
\text { Css }=R / V_{D} k=R / \text { Clearance }
$$

\section{N.B:}

1. At steady-state, the rate of drug input $(R)$ is equal to the rate of drug output $\left(k D_{P}\right)$,

so, $R=k D_{P}$ and $R=k \cdot C_{P} \cdot V_{D} C_{P}=R / V_{D} \cdot k$

At steady-state, $C_{\mathrm{P}}=C_{\mathrm{ss}}$

So, $R=k C_{s s} V_{D} C_{s s}=R / k \cdot V_{D} C_{s s}=R / C_{l}$

2. Once the infusion stops either this achieved at or before steady-state is reached, the drug concentration will decline according to first-order rate kinetics with the slope of the elimination curve that is equal to $-k / 2.3$.

\section{Example}

A desired steady-state plasma concentration of theophylline may be $15 \mathrm{mg} / \mathrm{L}$. The average half-life of theophylline is about $4 \mathrm{hr}$ and the apparent volume of distribution is about 25 liter. What is the necessary infusion rate?

From the $\mathrm{t}_{1 / 2}, \mathrm{k}_{\mathrm{el}}$ or $\mathrm{k}=0.693 / 4=0.17 \mathrm{hr}^{-1}$

$$
R=\operatorname{Css} k V_{D}=15 \times 0.17 \times 25=63.75 \mathrm{mg} / \mathrm{hr}
$$

\subsubsection{Steady-State Drug Concentration $\left(C_{S S}\right)$ and Time to Reach Steady-State}

During administration of a drug by IV infusion, the plasma drug concentration starts to increase and the rate of drug elimination will also increase since the latter is concentrationdependent. $C_{p}$ keeps increasing until a steady-state condition is reached at which the rate of drug input (IV infusion rate) equals the rate of drug output (elimination rate). At this stage, a steady-state $\left(C_{\mathrm{SS}}\right)$ is reached and the resulting plasma drug concentration is directly related to the rate of infusion and inversely related to the body clearance of the drug.

For drug administered by IV infusion, the therapeutic activity is observed when the concentration of the drug is close to the desired plasma concentration, which is usually the required steady-state drug concentration.

The time to reach steady-state could be determined by knowing the time to reach half the steady-state which can be derived: 
Since $C_{s s}=R / V_{D} k=R /$ Clearance

At $\left(t_{1 / 2}\right)$; time to reach half the steady-state $C_{\mathrm{P}}=C_{\mathrm{ss}} / 2$

$$
\begin{gathered}
C_{P}=\left[R / V_{D} \cdot k\right]\left(1-e^{-k t}{ }_{\text {half }}\right)=C_{s s} / 2 \\
C_{P}=C_{s s}\left(1-e^{-k t}{ }_{h a l f}\right)=C_{s s} / 2 \\
C_{s s}\left(1-e^{-k t}{ }_{\text {half }}\right)=C_{s s} / 2 \\
1-e^{-k t}{ }_{\text {half }}=1 / 2 \\
-e^{-k t}{ }_{\text {half }}=1 / 2-1 \\
e^{-k t}{ }_{\text {half }}=0.5
\end{gathered}
$$

Taking the $\ln$ to both sides, $t_{\text {half }}=0.693 / k$

It must be noticed that the time to reach half the steady-state has the same value for the elimination half-life and is dependent on the elimination process not the infusion rate while the value of $\mathrm{C}_{\mathrm{ss}}$ is controlled by the infusion rate. An increase in the infusion rate will not shorten the time to reach the steady-state drug concentration.

From the pharmacokinetic point of view, for drugs administered by IV infusion, the clinical effect of the drug (activity) is observed when the drug concentration in the plasma is close to the desired plasma drug concentration, which is the desired steady-state drug concentration.

\section{Examples}

1. A drug belonging to the cephalosporins antibiotics has a volume of distribution of $10 \mathrm{~L}$ and an elimination rate constant $(\mathrm{k})$ value of $0.2 \mathrm{hr}^{-1}$. A steady-state plasma concentration of $10 \mu \mathrm{g} / \mathrm{mL}$ is desired. What is the infusion rate required to maintain this concentration.

$$
R=C_{s s} \cdot V_{D} \cdot k \quad R=10 \times(10 \times 1000) \times 0.2=20 \mathrm{mg} / \mathrm{hr}
$$

2. A septicemia patient was administered by constant IV infusion an antibiotic that has an elimination half-life $\left(t_{1 / 2}\right)$ of $6 \mathrm{hr}$. The rate of infusion was $2 \mathrm{mg} / \mathrm{hr}$. At the end of second day of treatment, the serum drug concentration was $10 \mathrm{mg} / \mathrm{L}$. Calculate the total body clearance $\mathrm{Cl}_{\mathrm{T}}$ for this drug. 


$$
C_{s s}=R / C_{l} \quad C_{l}=R / C_{s s} \quad C_{l}=2 / 10=200 \mathrm{ml} / \mathrm{hr}
$$

3. An infinitely long period of time is needed to reach a steady-state level of a certain drug. However, in clinical practice it is quite acceptable to reach $99 \%$ of the steady-state level $\left(99 \% C_{s s}\right)$. Calculate a time required to reach steady-state relative to the half-life of this drug.

$$
C_{P}=\left[R / V_{D} k\right]\left(1-e^{-k t}\right)
$$

At $99 \%$ steady-state, $C_{P}=99 \%$ Css $=99 \% R / V_{D} k$

So, $\left[R / V_{D} k\right]\left(1-e^{-k t}\right)=99 \% R / V_{D} k$

$$
1-e^{-k t}=99 \%
$$

Taking the natural logarithm, $\ln 0.01=-k t$

$$
t_{99 \% s s}=\ln 0.01 /-k=4.69 / k
$$

Substitution for $\mathrm{k}=0.693 / \mathrm{t}_{1 / 2}$

$$
t_{99 \% \text { ss }}=4.61 /\left(0.693 / t_{1 / 2}\right)=6.65 t_{1 / 2}
$$

Accordingly, as a general example, if the $t_{1 / 2}$ for a drug is $6 \mathrm{hr}$, the time needed to reach $90 \%$, $95 \%$, and $99 \%$ of the steady-state is to be calculated. So, at those concentrations, $10 \%, 5 \%$, and $1 \%$ respectively dropped from the total will take 3.32, 4.32, and 6.65 half-lives. Thus, the time for a drug whose $t_{1 / 2}$ is $6 \mathrm{hr}$ to reach at least $95 \%$ of the steady-state plasma drug concentration will be $5 t_{1 / 2}$ or $5 \times 6 \mathrm{hr}=30 \mathrm{hr}$. Also, for theophylline with a $t_{1 / 2}$ equal to $4 \mathrm{~h}$, the time to reach $94 \%$ of steady-state will be $16 \mathrm{hr}$.

\subsubsection{Calculating Patient Elimination Half-Life Following Drug Infusion}

The half-life for drug administered by IV infusion could be calculated from (0.693/k). So, a mathematical expression that describes the elimination rate constant should first be determined.

$$
C_{P}=\left[R / V_{D} k\right]\left(1-e^{-k t}\right)
$$


And since $C_{s S}=R / k V_{D}$

$$
\begin{gathered}
C_{P}=\left[C_{s s}\right]\left(1-e^{-k t}\right) \\
C_{P}=C_{s s}-C_{s s} \cdot e^{-k t} \\
C_{s s} \cdot e^{-k t}=C_{s s}-C_{P} \\
e^{-k t}=\left(C_{s s}-C p\right) / C_{s s} \\
k t / 2.303=\log \left(C_{s s}-C p\right) / C_{s s} \\
k=-2.303 / t \cdot \log \left(C_{s s}-C p\right) / C_{s s}
\end{gathered}
$$

It is noteworthy to mention that knowing the half-life for the administered drug in the general population helps to determine if the sample is taken at steady-state in the patient, and after administration of the drug by the IV infusion, one or two plasma samples must be taken at a known time to compare.

\section{Examples}

A patient was administered an IV infusion of a certain antibiotic at an infusion rate of $15 \mathrm{mg} /$ $\mathrm{hr}$. Blood samples were taken from this patient at 8 and at $24 \mathrm{hr}$ and the plasma concentrations for this drug were found to be 5.5 and $6.5 \mathrm{mg} / \mathrm{L}$, respectively. Calculate approximately the elimination half-life of this drug.

N.B: The antibiotic has an elimination half-life of 3-6 hr in general population.

\section{Answer}

Assuming the extreme case of $\mathrm{t}_{1 / 2}(6 \mathrm{hr})$, the second plasma sample was taken at $24 \mathrm{hr}$, or 24/6 $=4$ half-lives after infusion, which is supposed to be near the theoretical time for steady-state.

$$
\begin{gathered}
k=[-2.303 / t] \log \left(C_{s s}-C_{p} / C_{s s}\right) k=[-2.303 / 8] \log (6.5-5.5 / 6.5) \\
k=0.234 h^{-1} \quad t_{1 / 2}=0.693 / 0.234=2.96 h r
\end{gathered}
$$

In the previous example, if we desire to let the steady-state plasma concentration equal $8 \mathrm{mg} /$ $\mathrm{L}$, what is the suitable infusion rate? 


\section{Answer}

First from the old data, we should calculate the clearance rate:

$$
\text { Css }=R / C_{l} \quad C_{l}=15 / 6.5=2.31 \mathrm{~L} / \mathrm{hr}
$$

Then, for the new infusion rate $R=\operatorname{Css} x \mathrm{Cl}=8 \times 2.31=18.48 \mathrm{mg} / \mathrm{hr}$

\subsubsection{Loading Dose plus IV Infusion (Combined Infusion and Bolus Administration)}

If we desire to achieve a quick therapeutic concentration, a loading dose by rapid intravenous injection (bolus injection) is first administered and then starts the slower maintenance infusion. At this condition, the total drug concentration in the plasma is the function of both; the IV bolus and the infusion doses.

The concentration following IV bolus $\left(\mathrm{C}_{1}\right)$ is described by:

$$
C_{1}=C_{o} e^{-k t}=\text { Loading Dose }\left(D_{L}\right) / V_{D} e^{-k t}
$$

The concentration following IV infusion at rate $\mathrm{R}$ is:

$$
C_{2}=R / V_{D} k\left(1-e^{-k t}\right)
$$

The total concentration $\mathrm{C}_{\mathrm{p}}$ will be the sum of bolus and infusion:

$$
\begin{gathered}
C_{P}=C_{1}+C_{2} \\
C_{P}=D_{L} / V_{D} e^{-k t}+R / V_{D} k \cdot\left(1-e^{-k t}\right) \\
\text { Or, } C_{P}=R / V_{D} k+\left(D_{L} / V_{D} \cdot e^{-k t}-R / V_{D} k \cdot e^{-k t}\right)
\end{gathered}
$$

If the loading dose $\left(D_{L}\right)$ represents or equals the amount of drug in the body at steady-state,

$$
\text { so, } D_{L}=C_{s s} \cdot V_{D} \text { and since } C_{s s}=R / V_{D} k \text {, so, } C_{s s} \cdot V_{D}=R / k
$$

Therefore, $D_{L}=R / k$

\section{Examples}


An anesthetic agent was administered by IV infusion at a rate of $2 \mathrm{mg} / \mathrm{hr}$. The drug has an elimination rate constant of $0.1 \mathrm{hr}^{-1}$, and a volume of distribution (one compartment) equals to $10 \mathrm{~L}$. What loading dose is recommended if the physician wants the drug level to reach 2 $\mu \mathrm{g} / \mathrm{mL}$ immediately?

\section{Solution}

$$
\begin{gathered}
D_{L}=C_{s s} V_{D} \quad D_{L}=2 \mu g / m L \times 10 \times 1000=20000 \mu g=20 m g \\
\text { Or, } D_{L}=R / k \quad D_{L}=2 / 0.1=20 m g
\end{gathered}
$$

A drug was given to a patient by simultaneous administration of a loading dose of $10 \mathrm{mg}$ and an infusion rate of $2 \mathrm{mg} / \mathrm{hr}$ (the drug has a $t_{1 / 2}$ of $3 \mathrm{hr}$ and a volume of distribution of $10 \mathrm{~L}$ )? What is the concentration of a drug following $6 \mathrm{hr}$ of administration?

\section{Solution}

$$
k=0.693 / 3=0.231
$$

The concentration of the drug $\left(\mathrm{C}_{\mathrm{P}}\right)$ after $6 \mathrm{hrs}=$ Loading dose $\left(\mathrm{C}_{1}\right)+$ Concentration by infusion $\left(\mathrm{C}_{2}\right)$

$$
\begin{gathered}
C_{P}=D_{L} / V_{D} \cdot e^{-k t}+R / V_{D} k \cdot\left(1-e^{-k t}\right) \\
C_{P}=10 \times 1000 / 10 \times 1000 \cdot e^{-(0.231 \times 6)}+[2 \times 1000 /(10 \times 1000)(0.231)] \cdot\left(1-e^{-(0.231 \times 6)}\right) \\
C_{P}=0.9 \mu \mathrm{g} / \mathrm{ml}
\end{gathered}
$$

N.B: To determine the concentration of the drug in the body after infusion has been stopped, first the final concentration of drug at the end of the infusion is to be calculated and considered as $C_{0}$. Then, use the IV bolus dose equation $\left(C=C_{0} e^{-k t}\right)$ for calculations for any further point in time.

A patient was infused for $6 \mathrm{hr}$ with a drug $\left(k=0.01 \mathrm{hr}^{-1} ; V_{\mathrm{D}}=10 \mathrm{~L}\right)$ at a rate of $2 \mathrm{mg} / \mathrm{hr}$. What is the concentration of the drug in the body $2 \mathrm{hr}$ after cessation of the infusion?

\section{Solution}

$$
C_{P}=R / V_{D} k \cdot\left(1-e^{-k t}\right)=2 \times 1000 / 10 \times 1000 \times 0.01 \cdot\left(1-e^{-0.01 \times 6}\right)
$$




$$
C_{P}=2\left(1-e^{-0.06}\right) \quad C_{P}=2-e^{-0.06}
$$

The obtained value for $C_{P}$ is to be considered the initial concentration of the drug in the plasma after cessation of the infusion in which the IV bolus equation could be applied:

$$
\begin{gathered}
C_{P}=C_{P}{ }^{0} \cdot e^{-k t} \quad C_{P}=2\left(1-e^{-0.06}\right) \cdot e^{-0.01 \times 2} \\
C_{P}=1.14 \mu \mathrm{g} / \mathrm{ml}
\end{gathered}
$$

1. A 35-year-old male patient of $80 \mathrm{~kg}$ was given a chemotherapeutic agent by IV infusion. The administered drug has been reported to have an elimination half-life $\left(t_{1 / 2}\right)$ of $2 \mathrm{hr}$, an apparent volume of distribution of $1.25 \mathrm{~L} / \mathrm{kg}$. If the effective plasma concentration of this drug is $14 \mathrm{mg} / \mathrm{l}$ and the drug is commercially supplied in ampoules of 5-mL each containing $150 \mathrm{mg} / \mathrm{mL}$ :

2. What is the recommended starting infusion rate in milligrams per hour and liters per hour?

3. After the start of the infusion, blood samples were taken from the patient at 12,16 , and 24 $\mathrm{hr}$ and the plasma drug concentrations were found to be $16.1,16.3$, and $16.5 \mathrm{mg} / \mathrm{L}$. Calculate the drug total body clearance $\mathrm{Cl}_{\mathrm{T}}$ in this patient.

4. Considering the data provided above, calculate approximately the elimination half-life for this antibiotic.

\section{Solution}

$$
k=0.693 / 2=0.3465
$$

Assume the effective plasma drug concentration is the target drug concentration or $C_{\mathrm{ss}}$ :

$$
R=C_{s s} k V_{D}=14 \times 0.3465 \times 1.5 \times 80=582.1 \mathrm{mg} / \mathrm{hr},
$$

which is equivalent to $582.1 / 150=3.88 \mathrm{ml}$, since each ampoule contains $150 \mathrm{mg} / \mathrm{ml}$

It is obvious that the drug has reached a steady plasma level of approximately 16.3 (average value).

So, $C_{l}=R / C s s=582.1 / 16.3=35.7 \mathrm{~L} / \mathrm{hr}$

$$
C_{l}=k V_{D} \quad k=C l / V_{D} \quad k=35.7 / 1.25 \times 80=0.357 h^{-1}
$$




$$
t_{1 / 2}=0.693 / 0.357=1.94 h r
$$

\subsection{Intravenous Infusion of Two-Compartment Model Drugs}

Some drugs such as lidocaine and theophylline when administered by IV infusion do not follow the one- but rather the two-compartment model. As has been discussed in the IV bolus, during a constant IV infusion, the drug in the tissue compartment is in distribution equilibrium with that in the plasma and a steady state concentration is reached during equilibrium.

The mathematical expression that describes the drug plasma concentration is:

$$
C_{P}=R / V_{P} k\left[1-(k-b / a-b) e^{-a t}-(a-k / a-b) e^{-b t}\right]
$$

At steady-state $(t=\alpha)$, the last equation could be reduced into:

$$
\text { CsS }=R / V_{P} k
$$

N.B: It is not possible to maintain a steady-state stable blood level for a drug that exhibits a two-compartment model drug following a zero-order rate of infusion. Therefore, a loading dose is administered to produce an initial plasma level either slightly higher or lower than that of the desired steady-state level. Several IV bolus injections may be given as short intermittent IV infusions as a method for administering a loading dose to the patient

\subsubsection{Apparent Volume of Distribution at Steady State, Two-Compartment Model}

The amount of the drug in the body at steady-state is the product of the plasma concentration and the steady-state volume of distribution $\left(\mathrm{V}_{\mathrm{D}}\right)$ ss. Assuming equilibrium has been attained between the central and peripheral compartments:

so, $D_{t} k_{21}=D_{P} k_{12} D t=k_{12} D_{P} / k_{21} D t=k_{12} C_{P} V_{P} / k_{21}$

At the steady-state, the total amount of drug in the body is equal to the sum of the amount of drug in tissue $\left(D_{\mathrm{t}}\right)$ and central compartment $\left(D_{\mathrm{p}}\right)$. Consequently, $\left(V_{\mathrm{D}}\right)_{\mathrm{SS}}$ could be determined by dividing the total amount of drug in the body by the concentration of drug in the central compartment at steady-state:

$$
\begin{gathered}
\left(V_{D}\right) S S=D_{P}+D_{t} / C_{P} \quad\left(V_{D}\right) S S=\left[C_{P} V_{P}+k_{12} V_{P} C_{P} / k_{21}\right] / C_{P} \\
\left(V_{D}\right) S S=V_{P}+k_{12} / k_{21} V_{P}
\end{gathered}
$$




\section{Pharmacokinetics of Drugs Following Oral Absorption}

Unlike the process of IV administration, when a drug is introduced into the body by extravascular route such as oral, intramuscular, subcutaneous, or transdermal administration, an absorption phase that transfers the drug from the absorption site into the systemic vascular system must take place. The oral route of drug administration represents the most popular of the extravascular routes and so, in this chapter, we will focus on its pharmacokinetics; although, the same principles could be applied for the other extravascular routes. The plasmalevel time curve for drugs administered by intramuscular, subcutaneous, or transdermal showed the same profile for orally administered drugs. Also, it is important to mention that for the same route, different drug formulations such as oily, aqueous liquid, suspension, emulsion, semisolid, fast dissolving tablets, oral disintegrating tablets, or buccal tablets exhibit the same plasma time curve but with slight modification in the rate and extent of absorption and/or distribution phases [8]. This concept is common during relative bioavailability study that involves identification of a significant difference between different pharmaceutical products administered by the same or another non-intravenous route of administration.

As previously mentioned, the process of gastrointestinal (GIT) absorption is mainly dependent on physiology and anatomy of the GIT, physicochemical factors of the drug, and dosage-formrelated factors $[1,3]$.

The process of oral drug absorption (drug input) is mainly first-order, unless it has been verified experimentally or through the pharmacokinetic models that it is zero-order.

The figure below represents the plasma-level time curve for a drug administered by oral route; unlike the IV bolus, there is an absorption phase and the overall rate of change in the amount of the drug in the body $\mathrm{dD}_{\mathrm{B}} / \mathrm{dt}$ is the function of both the rate of drug absorption and elimination. The maximum plasma concentration is represented as $C_{\max }$ and the time needed to reach maximum concentration is represented as $t_{\max }$.

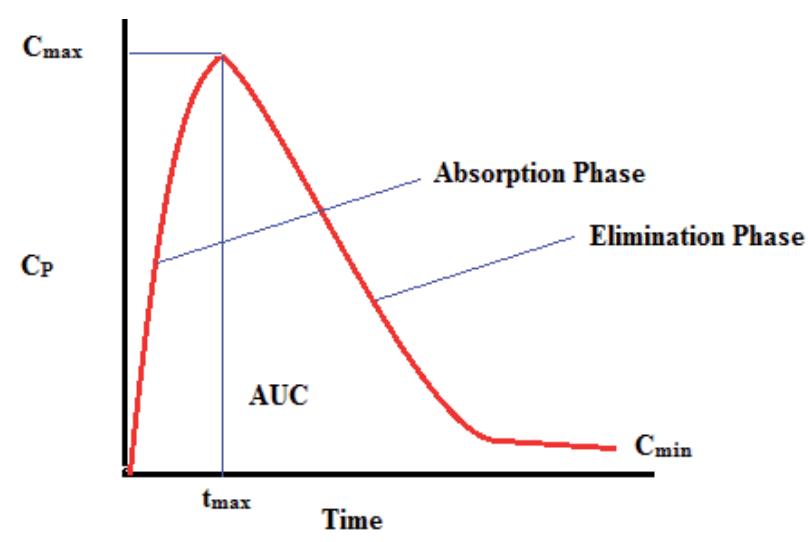

Figure 10. Plasma-level time curve for a drug administered by oral route 
If $\mathrm{D}_{\mathrm{GI}}$ represents the amount of the drug in the GIT and $\mathrm{D}_{\mathrm{E}}$ represents the amount of the drug eliminated, the change in the amount of the drug in the body $\mathrm{dD}_{\mathrm{B}} / \mathrm{dt}$ could be expressed as:

$$
d D_{B} / d t=d D_{G I} / d t-d D_{E} / d t
$$

The above curve could be classified into three segments (phases):

- The absorption phase, where the rate of drug absorption $\left(\mathrm{dD}_{\mathrm{GI}} / \mathrm{dt}\right)$ is greater than the rate of drug elimination $\left(\mathrm{dD}_{\mathrm{E}} / \mathrm{dt}\right)$

- The peak drug plasma concentration, in which the rate of drug absorption $\left(\mathrm{dD}_{\mathrm{GI}} / \mathrm{dt}\right)$ is equal to the rate of drug elimination $\left(\mathrm{dD}_{\mathrm{E}} / \mathrm{dt}\right)$

- The elimination phase, when the rate of drug elimination $\left(\mathrm{dD}_{\mathrm{E}} / \mathrm{dt}\right)$ is greater than the rate of drug absorption $\left(\mathrm{dD}_{\mathrm{GI}} / \mathrm{dt}\right)$

It must be noted that, during the absorption phase and once the drug is available in the plasma, some of the absorbed drug may be eliminated, but absorption is major. Also, during the elimination phase, some drug may still remain at the absorption site; however, the rate of drug elimination is higher than the rate of drug absorption until the entire drug at the absorption site is depleted and hence, $\mathrm{dD}_{\mathrm{GI}} / \mathrm{dt}=$ zero and at this stage $\mathrm{dD}_{\mathrm{B}} / \mathrm{dt}=-\mathrm{dD}_{\mathrm{E}} / \mathrm{dt}$.

\subsection{Zero-Order Absorption Model}

It is previously mentioned that the oral drug absorption process is mainly first-order. Zeroorder absorption of drug is expected in two cases:

- Drugs absorbed through carrier-mediated transport

- The absorption of controlled release drug dosage forms

In this process, the drug is absorbed at a constant rate $\mathrm{k}_{\mathrm{o}}$ and is eliminated by first-order rate process in a model similar to that of the intravenous infusion.

The mathematical expression for this model is:

$d D_{B} / d t=k_{o}-k D_{B}$ and $d D_{B} / d t=k_{o}-k V_{D} C_{P}$

Integration of the above equation:

$$
C_{P}=k_{o} /\left[V_{D} \cdot k\left(1-e^{-k t}\right)\right]
$$

\subsection{First-Order Absorption Model}

Drugs absorbed by passive diffusion transport mechanism exhibit first-order absorption. Such behavior is expected from:

- Drugs administered as solution and most suspension 
- Immediate release tablets, capsules, and suppositories

- Intramuscular and subcutaneous aqueous injections

For any orally absorbed drug, if $(\mathrm{F})$ is the fraction of drug absorbed, $\mathrm{D}_{\mathrm{GI}}$ is the amount of drug in solution in the GIT at any time ( $t), \mathrm{k}_{\mathrm{a}}$ is the first-order absorption rate constant and $\mathrm{k}$ is the first-order elimination rate constant; therefore:

The rate of drug disappearance from GIT is: $d D_{G I} / d t=-k_{a} . D_{G I} . F$

The rate of first-order elimination process is: $d D_{E} / d t=-k . D_{B}$

The rate of the amount of drug changed in the body is: $d D_{B} / d t=F \cdot k_{a} \cdot D_{G I}-k \cdot D_{B}$

Since drug absorption is first-order, the amount of drug in the GIT $\left(\mathrm{D}_{\mathrm{GI}}\right)$ at any time $t$ is equal to $D_{o}$. $e^{-k a t}$, in which $D_{o}$ is the dose of drug given.

So,

$$
d D_{B} / d t=F \cdot k_{a} \cdot D_{o} \cdot e^{-k a t}-k \cdot D_{B}
$$

The last equation could be integrated to give the general oral absorption equation as follow:

$$
C_{P}=\left[F \cdot k_{a} \cdot D_{o} / V_{D} \cdot\left(k_{a}-k\right)\right] \cdot\left(e^{-k t}-e^{-k a t}\right)
$$

It must be noted that the value of F may vary from 1 for completely absorbed drug to zero for drug that is fully unabsorbed.

The time needed to reach the maximum plasma concentration $\mathbf{t}_{\max }$ could be calculated by considering that at $\mathrm{C}_{\max }$ (maximum plasma concentration), the rate of drug absorbed is equal to the rate of drug eliminated:

$$
\begin{gathered}
d D_{B} / d t=F \cdot k_{a} \cdot D_{G I}-k \cdot D_{B}=0 \\
F \cdot k_{a} \cdot D_{G I}=k \cdot D_{B} \\
F \cdot k_{a} \cdot D^{o} \cdot e^{-k a t}=k \cdot D^{o} \cdot e^{-k t} \\
k_{a} e^{-k a t}=k e^{-k t} \\
\ln k_{a}-k_{a} t=\ln k-k t \\
\ln k_{a}-\ln k=k_{a} t-k t \\
\ln k_{a}-\ln k=t\left(k_{a}-k\right)
\end{gathered}
$$




$$
\begin{gathered}
t=\left(\ln k_{a}-\ln k\right) / k_{a}-k \\
t_{\max }=\left[2.303 \log \left(k_{a} / k\right)\right] / k_{a}-k
\end{gathered}
$$

From the above equation, $\mathrm{t}_{\max }$ is independent of the dose and is dependent on the rate constants for absorption $\left(\mathrm{k}_{\mathrm{a}}\right)$ and elimination $(\mathrm{k})$.

The value of $\mathbf{C}_{\max }$ is better to obtain mathematically (kinetically) since measurement of $C_{\max }$ may not be possible due to improper timing of the blood samples.

$\mathrm{C}_{\max }$ is determined first; calculate $\mathrm{t}_{\max }$ using the last equation:

$$
\left.t_{\max }=\left[2.303 \log \left(k_{a} / k\right)\right] / k_{a}-k\right]
$$

Then, use the obtained $t_{\max }$ value and substitute in the $C_{P}$ equation to get $C_{\max }$ in which $C_{P}=\left[F \cdot k_{a} \cdot D_{o} / V_{D}\left(k_{a}-k\right)\right]\left(e^{-k t}-e^{-k a t}\right)$

The value for the first-order elimination rate constant $(\mathbf{k})$ could be determined graphically from the plasma-level time curve after single oral administration, in which at later time, when the drug absorption has been completed, the value $\mathrm{e}^{\text {-kat }} \approx$ zero and plasma concentration equation will be:

$C_{P}=\left[F k_{a} D_{o} / V_{D}\left(k_{a}-k\right)\right]\left(e^{-k t}\right)$, which is analogue to $C_{P}=C_{P}^{o} \cdot e^{-k t}$

And so, $\log C_{P}=\log \left[F \cdot k_{a} . D_{o} / V_{D}\left(k_{a}-k\right)\right]-k t / 2.303$

If the log drug plasma concentration is plotted against time, a straight line for the elimination phase is obtained, from which the slope of the line is $-\mathrm{kt} / 2.303$

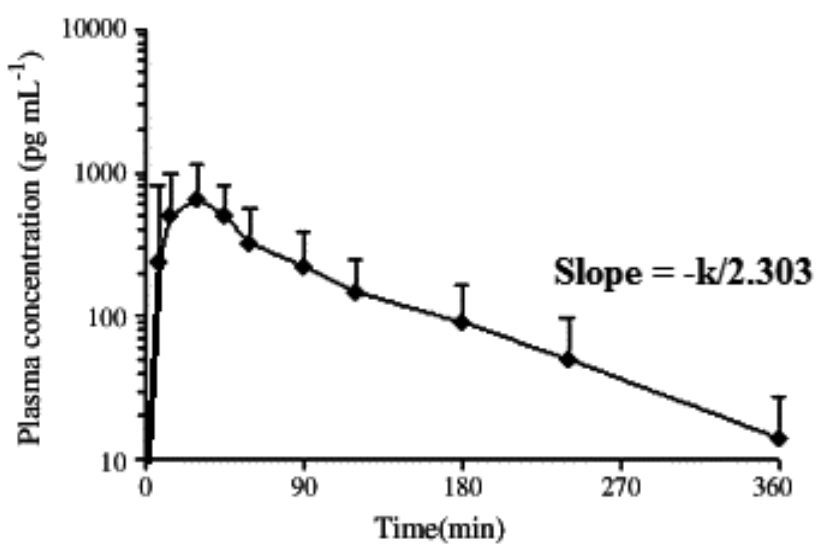

Figure 11. Semi-log plot of plasma concentration against time curve following oral adminstartion. 
The urinary drug excretion data could be used also to determine the first-order elimination rate constant in the same manner in which the kinetic process could be expressed as:

$$
d D_{u} / d t=\left[F \cdot k_{a} \cdot k_{e} \cdot D_{o} /\left(k_{a}-k\right)\right] \cdot\left(e^{-k t}-e^{-k a t}\right)
$$

A plot of $\mathrm{dD}_{\mathrm{u}} / \mathrm{dt}$ versus time will give a curve similar to the above curve, in which also after drug absorption has been completed, the value $-\mathrm{e}^{-\mathrm{ka}} \approx$ zero and a straight line for the terminal part is obtained, from which the slope of the line is $-\mathrm{kt} / 2.303$.

\subsection{Determination of Absorption Rate Constant ( $\left.\mathbf{k}_{\mathrm{a}}\right)$; Method of Residual}

The value for $\mathrm{k}_{\mathrm{a}}$ cannot be obtained directly from the plasma-level time curve for the drug administered by oral route as we calculated the elimination rate constant $(\mathrm{k})$, but it could be obtained using the method of residuals or a feathering technique, which is illustrated in the following graph:

1. On a semilog paper, plot the relation between plasma drug concentrations versus time.

2. Extrapolate the linear part of the terminal phase.

3. Identify a minimum of three points on the upper part of the extrapolated line $\left(X_{1}{ }^{\prime}, X_{2}{ }^{\prime}\right.$, and $\left.\mathrm{X}_{3}{ }^{\prime}\right)$.

4. From each identified point, drop a vertical line on the curve to obtain their corresponding points on the curve $\left(X_{1}, X_{2}\right.$, and $\left.X_{3}\right)$.

5. Plot again the values for the differences $\left(X_{1}{ }^{\prime}-X_{1}\right),\left(X_{2}{ }^{\prime}-X_{2}\right)$ and $\left(X_{3}{ }^{\prime}-X_{3}\right)$ versus time.

6. A straight line will be obtained in which the slope $=-\mathrm{k}_{\mathrm{a}} / 2.303$.

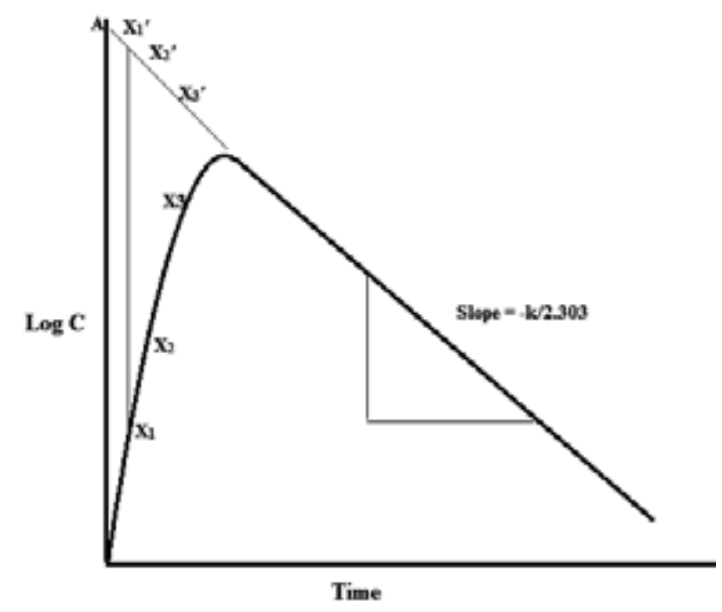

Figure 12. The plasma-level time curve for a drug administered by oral route used to determine $\mathrm{k}_{\mathrm{a}}$ by the method of residual 


\subsection{Lag time}

Some physiological factors such as gastric-emptying rate and motility of the intestine may cause delay in the oral absorption after single oral administration of some drugs in some candidates. In such case, there is a gap or a delayed period before the appearance of the first-order plasmalevel time curve as shown in the following figure, where the two residual lines obtained by feathering the oral absorption plasma-level time curve intersect at a point greater than $t=0$ on the $x$ axis.

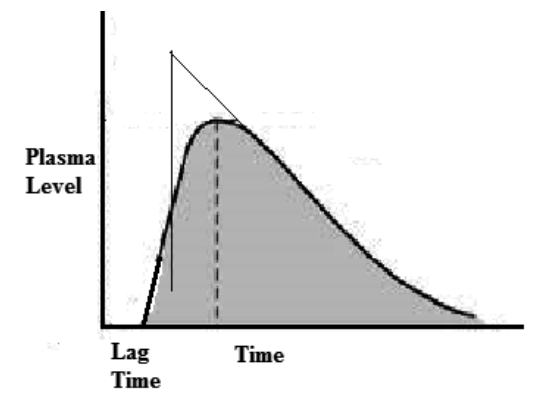

Figure 13. First-order plasma level time curve showing lag time

The drug plasma concentration can be described in this case as:

$$
C_{P}=\left[F \cdot k_{a} \cdot D_{o} / V_{D}\left(k_{a}-k\right)\right]\left(e^{-k(t-t o)}-e^{-k a(t-t o)}\right)
$$

\subsection{Flip-Flop of $\mathrm{k}_{\mathrm{a}}$ and $\mathrm{k}$}

According to the previous discussion for drugs administered by single oral dose, the absorption phase is much faster than the elimination phase and so, $\mathrm{k}_{\mathrm{a}}>\mathrm{k}$. It has been observed with some drugs such as isoproterenol and salicyluric acid that the elimination rate of these drugs is much greater than their absorption rate, such drugs exhibit flip-flop phenomenon (reversal or interchange of the values of the rate constants). To make sure of this behavior, the drug is administered by oral and IV bolus routes and elimination rate $\mathrm{k}$ is calculated for both routes; then the absorption rate $\mathrm{k}_{\mathrm{a}}$ is determined for oral route by the method of residual. It will be obvious that the values for $\mathrm{ka}$ and $\mathrm{k}$ obtained by the method of residuals have been interchanged. Such behavior is expected from drugs that have a fast elimination rate in which $\mathrm{k}>$ $\mathrm{k}_{\mathrm{a}}$ and from controlled release products.

\subsection{Determination of $k_{a}$ by Plotting Percent of Drug Unabsorbed versus Time (Wagner- Nelson Method)}

For any drug administered as a single oral dose $\left(D_{o}\right)$, after an elapsed time $(t)$, there will be an amount unabsorbed or remains in the GIT $\left(D_{\mathrm{GI}}\right)$, amount will be excreted in the urine $(\mathrm{Du})$ and an amount will be available in the body plasma $\left(\mathrm{D}_{\mathrm{B}}\right) ; D_{o}=D_{G I}+D u+D_{B}$

- The amount of the drug absorbed $(\mathrm{Ab})=\mathrm{Du}+\mathrm{D}_{\mathrm{B}}$ 
- The fraction of the drug remaining (unabsorbed) in the GIT is $\mathrm{D}_{\mathrm{G}} / \mathrm{D}_{\mathrm{o}}$

The amount of the drug remaining in the GIT (unabsorbed) at any time ( $t$ ) could be expressed by:

$$
\begin{gathered}
d D_{G I} / d t \alpha D_{o} \quad d D_{G I} / d t=k_{a} D_{o} \\
D_{G I}=D_{o} e^{-k a t} \\
D_{G I} / D_{o}=e^{-k a t} \quad \log D_{G I} / D_{o}=-k_{a} \cdot t / 2.303
\end{gathered}
$$

Plotting the fraction of drug unabsorbed versus time in a semilog graph paper will yield a straight line, the slope of which is $\mathrm{k}_{\mathrm{a}} / 2.303$.

\subsection{Effect of $k_{a}$ and $k$ on $C_{\max }, t_{\max ,}$ and AUC}

It could be asked: Does the change in ka and $k$ affect the values of $t_{\max }, C_{\max }$ and AUC? The answer is illustrated in the following figures and it could be explained as follows:

1. If the value for $\mathrm{k}_{\mathrm{a}}$ increased $(0.3$ to $0.7 / \mathrm{hr})$ while the elimination rate is kept constant, then $t_{\max }$ becomes shorter, $C_{\max }$ increases, and AUC remains constant.

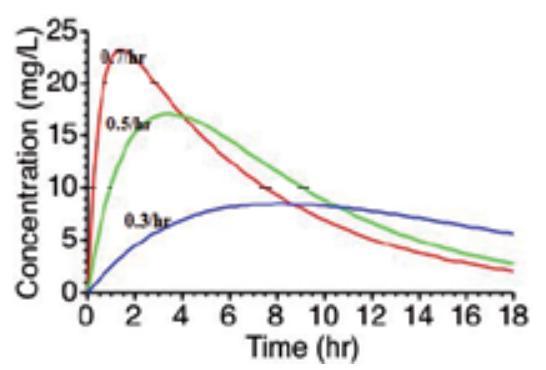

Figure 14. The plasma-level time curve for a drug administered by oral route showing change in ka and $\mathrm{k}$ and their effect on the values of $t_{\max }, C_{\max }$ and AUC

2. If the absorption rate is kept constant and the elimination rate increases ( 0.1 to $0.5 / \mathrm{hr})$, then $\mathrm{t}_{\max }$ decreases, $\mathrm{C}_{\max }$ decreases, and AUC decreases.

3. If the values for the absorption rate $k_{\mathrm{a}}$ and the elimination rate $k$ are reversed, then the same $t_{\max }$ is obtained while $C_{\max }$ and AUC are different.

\subsection{Drug Elimination}

The process of drug elimination from the body involves two major ways: metabolism/biotransformation (by the liver) and/or excretion (mostly by the kidney). The rate process for drug elimination is an overall first-order. 
The high blood supply to the liver, $20 \%$ arterial blood directly from the heart and $80 \%$ venous blood from the gastro-intestinal tract through the hepatic-portal vein (first-pass effect), and the liver microsomal enzymes make the liver the main site for drug metabolism. Oxidation, reduction, hydrolysis, and conjugation are the four metabolic processes.

Drug excretion is the removal of the drug from the body, which is mainly renal for nonvolatile, water-soluble, and low molecular weight drugs. Some other routes of drug excretion involve removal of the drug through bile, sweat, saliva, milk, or other body fluids.

Anatomically, the kidneys are paired retroperitoneal structures with the left kidney located somewhat more superior in position than the right. The outer zone of the kidney is called the cortex and the inner is called medulla.

The two kidneys represent about $0.5 \%$ of the total body weight, receive approximately $20-25 \%$ of the cardiac output, and serve endocrine and nonendocrine functions. Nonendocrine functions including filtration and excretion of metabolic waste products (urea and ammonia), regulation of necessary electrolytes, fluid and acid-base balance. In addition, the kidney has two endocrine functions: secretion of renin which regulates blood pressure and secretion of erythropoietin that stimulates red blood cell production.

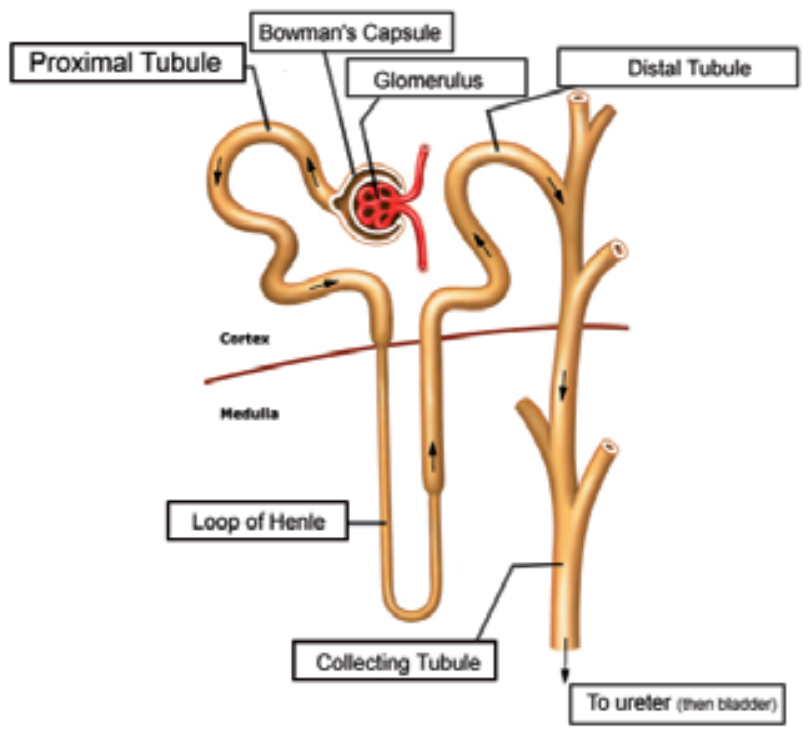

Figure 15. Structure of nephron

The nephrons are the basic functional units of the kidney responsible for the removal of metabolic waste and maintenance of water and electrolyte balance. There are 1-1.5 million nephrons in each kidney.

Each nephron is composed of a renal corpuscle and a renal tubule specialized for reabsorption and secretion. The renal corpuscle is composed of a glomerulus inside a bowman's capsule, 
which is the nephron's initial filtering component. The renal tubule, containing the tubular fluid filtered through the glomerulus, consists of proximal convoluting tubule, loop of henle (descending and ascending limb), and distal convoluting tubule. After passing through the renal tubule, the filtrate continues to the collecting duct, which is not part of the nephrons.

The volume of blood flowing through the kidney (renal vasculature) per unit time is referred to as the renal blood flow (RBF), which exceeds $1.2 \mathrm{~L} / \mathrm{min}$ or $1700 \mathrm{~L} /$ day, while, the renal plasma flow (RPF) is the renal blood flow minus the volume of red blood cells.

N.B: Hematocrit (Hct) is the fraction of blood cells in the blood which is about $45 \%$ of the total blood volume.

$$
R P F=R B F(1-H c t)
$$

The glomerular filtration rate (GFR) in a normal adult male subject is approximately $125 \mathrm{ml} /$ min, so about $180 \mathrm{~L}$ of fluid per day is filtered through the kidney. The average urine volume is $1-1.5 \mathrm{~L}$, which accounts for the reabsorption of $99 \%$ of the fluid volume filtered at the glomerulus.

\subsection{Renal Drug Excretion}

The mechanisms of renal drug excretion may include any combination of the following:

1. Glomerular filtration of unbound drug

2. Active tubular secretion of free and protein-bound drug

3. Tubular passive/active reabsorption

Glomerular filtration: Most drugs (ionized or nonionized) are readily filtered from the blood unless they are tightly bound to large molecules such as plasma protein or have been incorporated into red blood cells. The hydrostatic pressure within the glomerular capillaries is the major driving force for glomerular filtration. Inulin and creatinine are examples of drugs that are eliminated by filtration only and that could be used to measure the GFR.

Active tubular secretion: This process involves transport of the drug against a concentration gradient from the blood capillaries around the nephron to the nephron lumen, which is characterized by the following: it is carrier-mediated, requires energy, the carrier is saturable, and competition for the same carrier could occur. Iodopyracet (Diodrast) and $p$-aminohippuric acid (PAH) are common examples of drugs used to measure active tubular secretion, since both are filtered by the glomeruli and secreted by the tubular cells.

N.B: The process of drug protein binding has very little effect on the elimination $t_{1 / 2}$ of the drug excreted mostly by active secretion, while for drugs that are excreted only by glomerular filtration, the elimination half-life may be changed markedly according to the binding affinity of these drug for plasma proteins. Since drug protein binding is a reversible process, drug bound to plasma protein rapidly dissociates into a free drug that is secreted by kidneys. This 
behavior is very common for some of the penicillins that are extensively bound to plasma protein, but their elimination half-lives are short, which could be attributed to rapid elimination by active secretion.

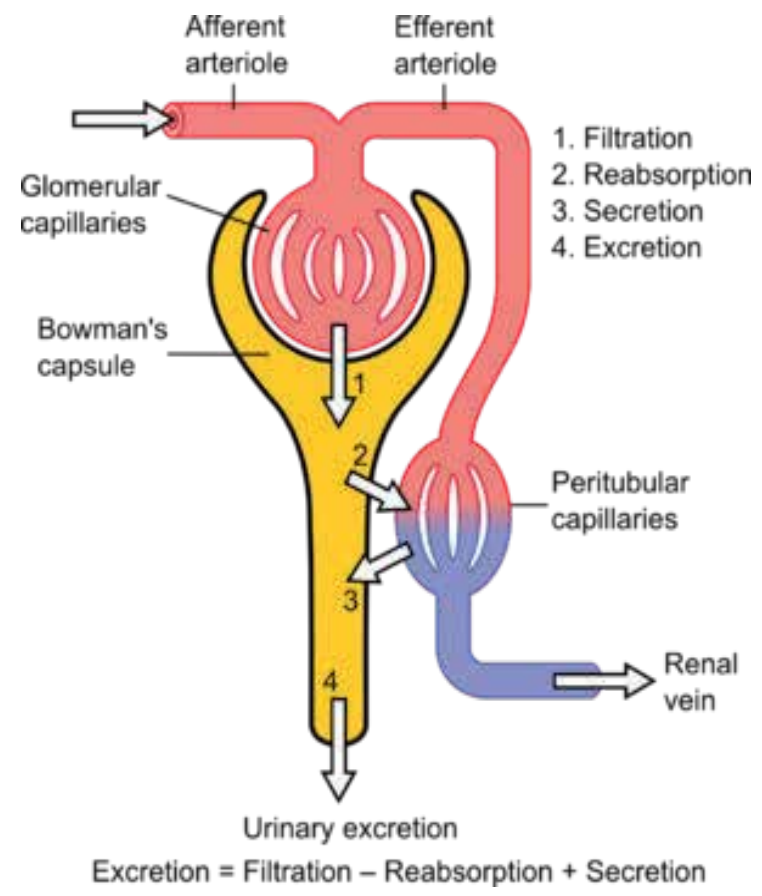

Figure 16. Mechanisms of renal drug excretion through nephron

Tubular reabsorption: Drug moves from nephron lumen to blood by passive diffusion (Fick's Law is applied) or sometimes by an active process. Most drugs are weak acids or weak bases and hence the reabsorption of such drugs is influenced by the $\mathrm{pH}$ of the renal tubule fluid and the drug pKa, as both will affect the ratio of dissociated (ionized) to the undissociated (nonionized). The drug $\mathrm{pK}_{\mathrm{a}}$ is constant, but the normal urinary $\mathrm{pH}$ may vary from 4.5 to 8.0, depending on diet, pathophysiology, and drug intake. The undissociated drug is easily reabsorbed from the renal tubule back to the body.

Some drugs such as ascorbic acid and antacids, for example, sodium carbonate, may alter the urine $\mathrm{pH}$. The former decreases (acidify) while the latter increases (alkalinize) the urinary $\mathrm{pH}$ when administered in large quantities. During excretion of these drug solutions, the urine $\mathrm{pH}$ may drastically change and alter other drug reabsorption and/or excretion by the kidney.

According to the Henderson-Hesselbalch equation, the percent of ionized to nonionized drug is the function of both $\mathrm{pH}$ and $\mathrm{pKa}$, as follows:

For acidic drugs: $p H=p K a+\log [$ ionized / unionized $]$ 


$$
\begin{gathered}
{[\text { Ionized / unionized }]=10^{(p H-p K a)}} \\
\text { Ionized }=[\text { unionized }] \cdot 10^{(p H-p K a)} \\
\text { *The fraction of acidic drug ionized }=[\text { ionized } /(\text { ionized }+ \text { unionized })] \\
=[\text { unionized }] \cdot 10^{(p H-p K a)} /\left\{[\text { unionized }] \cdot 10^{(p H-p K a)}+[\text { unionized }]\right\} \\
=10^{(p H-p K a)} / 1+10^{(p H-p K a)}
\end{gathered}
$$

For basic drugs: $p H=p K a+\log \left[\frac{\text { unionized }}{\text { ionized }}\right]$

${ }^{*}$ The fraction of basic drug ionized $=1+10^{(p H-p K a)} / 10^{(p H-p K a)}$

The urine-plasma $(\mathrm{U} / \mathrm{P})$ ratio of weak acidic or basic drugs could be evaluated from the following:

For weak acids: $U / P=1+10^{(p H \text { urine }-p K a)} / 1+10^{(p H \text { plasma }-p K a)}$

For weak bases: $U / P=1+10^{(p K a-p H \text { urine })} / 1+10^{(p K a-p H}$ plasma $)$

\section{Example}

The $\mathrm{pK}_{\mathrm{a}}$ of a weak acidic drug is 5 . Calculate the $\mathrm{U} / \mathrm{P}$ at urinary $\mathrm{pH}$ of 3,5 , and 7 , respectively.

\section{Solution}

For acidic drug, $U / P=1+10^{(p H \text { urine }-p K a)} / 1+10^{(p H \text { plasma }-p K a)}$

\begin{tabular}{lll}
\hline At $\mathrm{pH}=3$ & At $\mathrm{pH}=5$ & At $\mathrm{pH}=7$ \\
$\mathrm{U} / \mathrm{P}=1 / 252$ & $\mathrm{U} / \mathrm{P}=2 / 252$ & $\mathrm{U} / \mathrm{P}=101 / 252$ \\
\hline
\end{tabular}

\subsection{Drug Clearance}

As we mentioned for IV bolus administered drugs, Clearance or drug clearance is a pharmacokinetic term describing the process of drug elimination from the body without identifying the mechanism of this process. It refers to the volume of plasma fluid that is cleared of drug per unit time (volume approach, $\mathrm{L} / \mathrm{hr}$ or $\mathrm{ml} / \mathrm{hr}$ ) or the amount of the drug eliminated from the 
body per unit time (mass approach, $\mathrm{mg} / \mathrm{min}$ or $\mathrm{mg} / \mathrm{hr}$ ). It may also be considered as the fraction of the drug $V_{\mathrm{D}}$ that is excreted by the kidney per unit of time (fraction approach).

Practically, drug clearance (body clearance, total body clearance, or $\mathrm{Cl}_{\mathrm{T}}$ ) may also be expressed based on the plasma drug concentration as the elimination rate of the drug divided by the plasma drug concentration.

$$
\begin{aligned}
& C l_{T}=\frac{\text { Elimination rate }}{\text { Plasmaconcentration }(C p)}=\left(d D_{E} / d t\right) / C_{P} \\
& =\frac{\mu g / m i n}{\mu g / m l}=\mathrm{ml} / \mathrm{min}
\end{aligned}
$$

Since the drug elimination rate is a first-order process, so $d D_{\mathrm{E}} / d t$ is equal to $k D_{\mathrm{B}}$ or $k C_{\mathrm{p}} V_{\mathrm{D}}$.

$$
C l_{T}=k \cdot D_{B} / C_{P}=k \cdot C_{P} \cdot V_{D} / C_{P}=k \cdot V_{D}
$$

\section{Example}

Calculate the elimination rate for penicillin if the plasma drug concentration is $2 \mu \mathrm{g} / \mathrm{ml}$, assuming penicillin has a clearance of $15 \mathrm{ml} / \mathrm{min}$.

\section{Solution}

$$
\text { Elimination rate }=C_{P} x C l_{T}=2 \times 15=30 \mu \mathrm{g} / \mathrm{min}
$$

Using the previous penicillin example, assume that the plasma penicillin concentration is 10 $\mu \mathrm{g} / \mathrm{ml}$. Calculate the rate of drug elimination.

$$
d D_{E} / d t=10 \times 15=150 \mu g / \min
$$

\section{Example}

A 70-kg male patient has been administered a single oral dose of an antimalarial drug as a prophylactic treatment. If this drug has an elimination half-life of $3 \mathrm{hr}$ and an apparent volume of distribution of $100 \mathrm{~mL} / \mathrm{kg}$, assuming that this drug follows a one-compartment kinetic model, determine the drug total body clearance in this patient.

\section{Solution}

$$
k=0.693 / t_{1 / 2}=0.693 / 3=0.231 h r^{-1}
$$

$C_{T}=k V_{D}=0.231 \times 100=23.1 \mathrm{ml} / \mathrm{kg} \mathrm{hr}$ and for $70 \mathrm{~kg}, C l_{T}=23.1 \times 70=1617 \mathrm{ml} / \mathrm{hr}$ 


\subsection{Physiologic/Organ Clearance}

This term deals with any individual organ or tissue group involved in the process of drug removal from the body. If $\mathrm{Q}$ is the blood flow through this organ, $\mathrm{Ca}$ is the concentration of drug entering the organ (usually arterial drug concentration), $\mathrm{Cv}$ is the drug concentration leaving the organ (venous drug concentration), so the diagram that best describes this process will be:

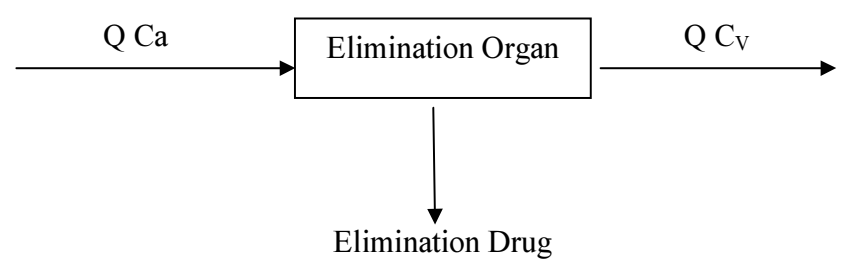

Figure 17. Diagram that best describes the process of physiologic/Organ Clearance

Accordingly, the physiologic/organ clearance may be described as the blood volume fraction which contains a specific drug that flows through the organ and is eliminated of this drug per unit time. Accordingly, clearance is considered as the product of the blood flow $(Q)$ to the organ, and the extraction ratio (ER):

Clearance $=$ Q. $($ ER)

ER is the fraction of drug extracted by the organ as drug passes through and is equal $\mathrm{Ca}-$ $\mathrm{C}_{\mathrm{V}}$ divided by the entering drug concentration $\mathrm{Ca}$, i.e., $E R=\left(\mathrm{Ca}-\mathrm{C}_{\mathrm{V}}\right) / \mathrm{Ca}$

$$
C l=Q \cdot\left[\left(C a-C_{V}\right) / C a\right]
$$

It must be noted that clearance measurements using the physiologic approach require measurements of blood flow and extraction ratio to a specified organ or group of tissue, which is not so easy, and invasive techniques are needed to obtain these data.

\subsection{Model-Independent Methods}

These are noncompartment model approaches that help to determine certain pharmacokinetic parameters such as drug clearance and bioavailability $(F)$. In these methods, no assumption for a specific compartment model is required to evaluate the data, which is the major advantage of these methods. In addition, no complicated methods are required to determine the studied pharmacokinetic parameters such as the volume of distribution and the elimination rate constant. These parameters can be calculated directly from the equation that best fits the plasma-drug-concentration time curve.

Clearance can be estimated directly from the plasma-time concentration curve by: 


$$
C l=D_{o} /[A U C]_{0}^{\alpha}
$$

where $\mathrm{D}_{\mathrm{o}}$ is the dose and $[A U C]_{0}^{\alpha}=t_{0}^{\alpha} C_{P} d t$.

Since $[A U C]_{0}^{\alpha}$ is calculated from the plasma-level time curve from 0 to infinity by the trapezoidal rule, no compartmental model is assumed.

In summary, clearances may be expressed mathematically as:

$$
\begin{gathered}
\qquad l_{T}=\frac{\text { Eliminationrate }}{\text { Plasmaconcentration }(C p)} \\
=k \cdot D_{B} / C_{P}=k \cdot C_{P} \cdot V_{D} / C_{P}=k \cdot V_{D} \\
\text { Physiologic or organ clearance }=Q(E R)=Q[(C a-C v) / C a] \\
\text { Noncompartmental clearance }=D o /[A U C]_{0}^{\alpha}
\end{gathered}
$$

\subsection{Renal Clearance}

Renal clearance utilizes the same concept for the clearance or the drug clearance previously illustrated, except that the drug is cleared by the kidney and so the volume, mass, or fraction approaches are also applicable here. It could be expressed also as the urinary drug excretion rate $(\mathrm{dDu} / \mathrm{dt})$ divided by the plasma drug concentration:

$$
C l_{R}=\text { excretion rate / plasma concentration }=(d D u / d t) / C p
$$

For any drug cleared by the kidney, the rate of drug passing through the kidney must equal the rate of drug excreted in the urine.

$$
C l_{R} x C_{P}=Q_{u} x C_{u}
$$

where $C l_{\mathrm{R}}$ is renal clearance, $C_{\mathrm{p}}$ is plasma drug concentration, $Q_{\mathrm{u}}$ is the rate of urine flow, and $C_{\mathrm{u}}$ is the urine drug concentration.

$$
C l_{R}=Q_{u} x C_{u} / C_{P}=\text { excretion rate } / C_{P}
$$

\subsection{Excretion Mechanism (Comparison of Drug Excretion Methods)}

Since the renal excretion is the urinary drug excretion rate $(\mathrm{dDu} / \mathrm{dt})$ divided by the plasma drug concentration, the expression could be rephrased to include the drug excretion mechanisms as follows: 


$$
\begin{aligned}
& C l_{R}=\text { excretion rate / plasma concentration }= \\
& (\text { Glomerular filtration rate }+ \text { tubular secretion rate }- \text { tubular reabsorption rate }) / C_{P}
\end{aligned}
$$

The excretion mechanism could be identified if the clearance value of the drug is compared to a well-recognized standard reference such as Inulin, which is a substance completely cleared through glomerular filtration only (clearance ratio). The following table illustrates identification of the mechanism:

\begin{tabular}{cc}
\hline Clearance Ratio & Renal Excretion Mechanism \\
\hline $\mathrm{Cl}$ drug $/ \mathrm{Cl}$ Inulin $<1$ & Drug is subjected to tubular reabsorption \\
\hline $\mathrm{Cl}$ drug $/ \mathrm{Cl}$ Inulin $=1$ & Drug is filtered only \\
\hline $\mathrm{Cl}$ drug $/ \mathrm{Cl}$ Inulin $>1$ & Drug is subjected to active tubular secretion \\
\hline
\end{tabular}

\section{Example}

If glomerular filtration at rate $125 \mathrm{ml} / \mathrm{min}$ is the sole elimination mechanism for gentamicin and kanamycin from the body, assuming gentamicin and kanamycin have $V_{D}$ of 10 and $20 \mathrm{~L}$, respectively, is there a difference in the drug clearances for each drug based on the classic and physiologic approaches? If not, describe pharmacokinetic parameters that could be used to differentiate between the two drugs.

\section{Solution}

Since the two drugs are eliminated from the body by glomerular filtration only, the classic and physiologic approaches will be the same and equal $125 \mathrm{ml} / \mathrm{min}$.

For gentamicin: $\mathrm{k}=\mathrm{Cl} / \mathrm{V}_{\mathrm{D}}=125 / 10000=0.0125 \mathrm{~min}^{-1}$ and $\mathrm{t}_{1 / 2}=0.693 / 0.0125=55.44 \mathrm{~min}$, while for kanamycin: $\mathrm{k}=125 / 20000=0.00625 \mathrm{~min}^{-1}$ and $\mathrm{t}_{1 / 2}=0.693 / 0.00625=110.88 \mathrm{~min}$.

So, there is an obvious difference between the elimination rate and half-life of the two drugs, although both have similar drug clearance.

\subsection{Determination of Renal Clearance by Graphical Methods}

It is possible to determine the renal clearance graphically by two methods:

1. If after administration of a drug, the drug excretion rate $(\mathrm{dDu} / \mathrm{dt})$ versus the plasma concentration $(\mathrm{Cp})$ is plotted, the drug excretion rate is the slope of the curve, since

$\mathrm{Cl}_{\mathrm{R}}=$ excretion rate/plasma concentration, but we must note that the slope will be large for drug that is excreted rapidly and will be of small value for drug that is excreted slowly through the kidney, i.e., the slope is smaller.

$$
\text { Slope }=\text { renal clearance }=(d D u / d t) / C_{P}
$$




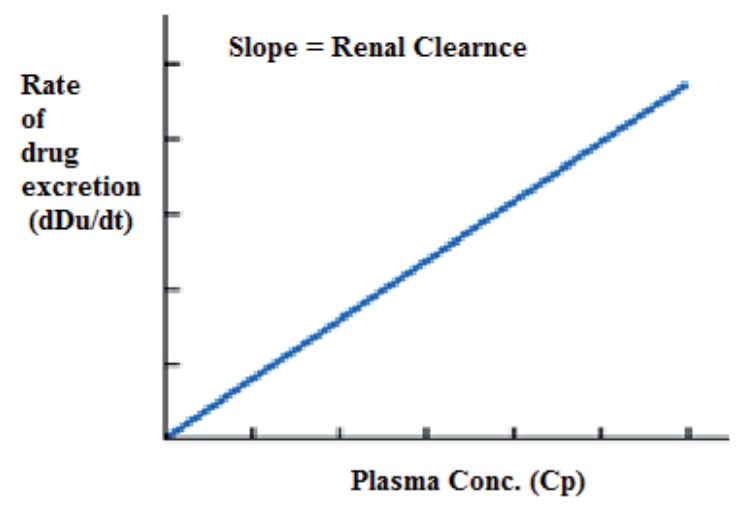

Figure 18. A plot of the drug excretion rate $\left(\mathrm{dD}_{\mathrm{u}} / \mathrm{dt}\right)$ versus the plasmaconcentration $\left(\mathrm{C}_{\mathrm{p}}\right)$

2. Since the renal clearance $C l_{R}=(d D u / d t) / C_{P}$, so $C l_{R} \cdot C_{P}=d D u / d t$

Rearranging and integration of the last equation will give:

$$
\int_{0}^{D u} d D u=C l_{R} \int_{0}^{t} C p d t \quad \text { So, }[D u]_{0}^{t}=C l_{R}[A U C]_{0}^{t}
$$

If a graph were plotted of the cumulative drug excreted in the urine versus the area under the plasma-level time curve, renal clearance is the slope of the curve.

By plotting cumulative drug excreted in the urine from $t_{1}$ to $t_{2},\left[D_{\mathrm{u}}\right]_{2 \mathrm{t} 1}^{\mathrm{t}}$ versus $[\mathrm{AUC}]_{2 \mathrm{t} 1}^{\mathrm{t}}$, an equation similar to that previously mentioned could be obtained, in which the slope is equal to the renal clearance: $\left[D_{u}\right]^{t 2_{t 1}}=C l_{R}[A U C]^{t_{2}}{ }_{t 1}$

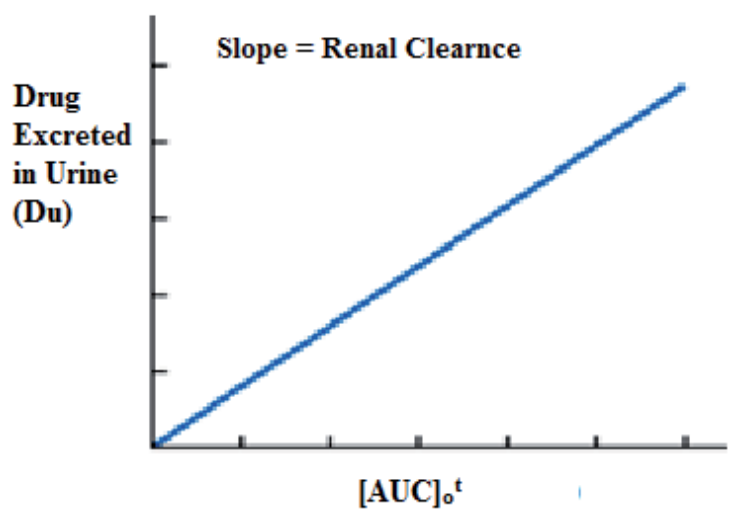

Figure 19. A plot of the cumulative drug excreted in the urine versus the area under the plasma-level time curve 


\section{Important Considerations}

1. Body clearance $\left(C l_{\mathrm{T}}\right)$, renal clearance $\left(C l_{\mathrm{R}}\right)$, and hepatic clearance $\left(C l_{\mathrm{h}}\right)$ can be calculated according to the following expressions:

$$
C l_{T}=k \cdot V_{D} \quad C l_{R}=k e \cdot V_{D} \quad C l_{h}=k m \cdot V_{D}
$$

2. Total body clearance $\left(\mathrm{Cl}_{\mathrm{T}}\right)$ is equal to the sum of renal clearance and hepatic clearance:

$$
C l_{T}=C l_{R}+C l_{h} \quad k V_{D}=k e V_{D}+k m V_{D} \quad k=k e+k m
$$

\subsection{Fraction of Drug Excreted}

The fraction of drug excreted unchanged in the urine $\left(\mathrm{f}_{\mathrm{e}}\right)$ is equal to the ratio of the total amount of unchanged drug excreted in the urine $D^{\alpha}{ }^{\alpha}$, to the fraction of the dose absorbed, $\mathrm{FD}_{0}$ :

$$
f_{e}=D^{\alpha}{ }_{u} / F D_{0}
$$

It was found that $\left(\mathrm{f}_{\mathrm{e}}\right)$ is also equal to $\mathrm{ke} / \mathrm{k}: f_{e}=D^{\alpha}{ }_{u} / F D_{0}=k_{e} / k$

\section{Problem}

I) A single oral dose of antibiotic (500 mg) was given. The drug is $90 \%$ systemically available. The total amount of unchanged drug recovered in the urine was found to be $300 \mathrm{mg}$, and the total amount of metabolite recovered in the urine was $150 \mathrm{mg}$. The drug has an elimination half-life of $3.3 \mathrm{hr}$ and its apparent volume of distribution is $1000 \mathrm{ml}$. Calculate the total body clearance, renal clearance, and nonrenal clearance.

\section{Solution}

1. $k=0.693 / 3.3=0.21 \mathrm{hr}^{-1} \mathrm{Cl}_{T}=k V_{D} C l_{T}=0.21 \times 1000=210 \mathrm{~mL} / \mathrm{hr}$

2. Since, $k e / k=D_{u}^{\alpha} /$ FDo $k e=(300 / 450)(0.21)=0.14 h r^{-1}$

$$
C l_{R}=k e V_{D}=0.14 \times 1000=140 \mathrm{~mL} / \mathrm{hr}
$$

3. $C l_{h}=C l_{T}-C l_{R} \quad C l_{h}=210-140=70 \mathrm{~mL} / \mathrm{hr}$

II) An analgesic drug was given to an elderly patient by IV bolus injection at a dose of $500 \mathrm{mg}$. The apparent volume of distribution and the elimination half-life of this drug were estimated to be $21 \mathrm{~L}$ and $6 \mathrm{hr}$, respectively. Urine samples were collected from the patient for $48 \mathrm{hr}$, and the amount of unchanged drug recovered was found to be $400 \mathrm{mg}$. Determine the fraction of the dose excreted unchanged from this drug in the urine. Calculate $k, k_{\mathrm{e}} \mathrm{Cl}_{\mathrm{T}}, \mathrm{Cl}_{\mathrm{R}}$, and $\mathrm{Cl}_{\mathrm{h}}$. 


\section{Solution}

$$
\begin{gathered}
f_{e}=400 / 500=0.8 \quad k=0.693 / 6=0.1155 \mathrm{hr}^{-1} \\
k e=f_{e} k=0.8 \times 0.115=0.0924 \mathrm{hr}^{-1} \\
C l_{T}=k V_{D}=0.1155 \times 21=2.34 \mathrm{~L} / \mathrm{hr} \\
C l_{R}=k e V_{D}=0.0924 \times 21=1.94 \mathrm{~L} / \mathrm{hr} \\
C l_{h}=C l_{T}-C l_{R}=2.34-1.94=0.49 \mathrm{~L} / \mathrm{hr}
\end{gathered}
$$

\section{Acknowledgements}

Some of the examples and information in this chapter have been abstracted partially from the book Applied Biopharmaceutics and Pharmacokinetics, but with major modifications that reflect the author's concept and his points of view.

\section{Author details}

Tarek A. Ahmed ${ }^{1,2^{*}}$

Address all correspondence to: dr_tarek_nour@yahoo.com

1 Department of Pharmaceutics and Industrial Pharmacy, Faculty of Pharmacy, King Abdulaziz University, Jeddah, KSA

2 Department of Pharmaceutics and Industrial Pharmacy, Faculty of Pharmacy, Al-Azhar University, Cairo, Egypt

\section{References}

[1] Leon Shargel, Susanna Wu-pong, Andrew B.C. Yu. (2004). Applied Biopharmaceutics and Pharmacokinetics (5th Edition). McGraw-Hill/Appleton \& Lange. ISBN: 0071375503.

[2] Loyd V. Allen, Nicholas G. Popovich, Howard C. (2004). Ansel. Ansel's Pharmaceutical Dosage Forms and Drug Delivery System (8th Edition). Lippincott Williams \& Wilkins. 
[3] Salvatore J. Turco, Robert E. King. (1994). Sterile Dosage Forms: Their Preparation and Clinical Application. (4th Revised Edition) Lippincott Williams \& Wilkins. Philadelphia, United States.

[4] Toutain PL, Bousquet-Mélou A. Volumes of distribution. J Vet Pharmacol Ther. 2004; 27(6): 441-53.

[5] Basic clinical pharmacokinetics, Page 32: Plasma protein binding By Michael E. Winter. (2003). Edition: 4, illustrated Published by Lippincott Williams \& Wilkins.

[6] Clark, B (1986). In Clark B, Smith D A, eds. An Introduction to Pharmacokinetics, 2nd ed. Oxford: Blackwell Scientific.

[7] Evans W E, Schentag J J, Jusko W J, Harrison H, eds (1992). In Evans W E, Schentag J J, eds. Applied Pharmacokinetics: Principles of Therapeutic Drug Monitoring, 3rd edn. Vancouver: Applied Therapeutics.

[8] Ahmed T.A., El-Say K.M., Mahmoud M.F., Samy A.M., Badawi A.A. (2012). Miconazole nitrate oral disintegrating tablets: in vivo performance and stability study. AAPS PharmSciTech. 13:760-71. 


\title{
Chapter 4
}

\section{Drug Distribution and Drug Elimination}

\author{
Seng Kok-Yong and Lee Lawrence \\ Additional information is available at the end of the chapter \\ http://dx.doi.org/10.5772/59929
}

\section{Introduction}

Pharmacokinetics is a branch of pharmacology that examines how drug concentrations change with respect to time as a function of absorption, distribution, metabolism and excretion [1]. These are disparate but interrelated processes that occur between drug administration and its irreversible elimination from the body. Another way to consider pharmacokinetic processes is to group them into two components:

1. intake, which describes the time course of drug movement from the site of administration, e.g. mouth, to the site of measurement, e.g. blood.

2. disposition, which describes the time course of drug distribution and elimination from the site of measurement e.g. blood.

Once absorbed into the body, drug compounds are distributed reversibly to various tissues of the body including the eliminating organs, such as liver and kidney, which results in a decrease in blood or plasma drug concentration. The decrease in the blood concentration could be due to reversible loss of drug from the blood to the tissues, defined as distribution, or the irreversible loss of drug from blood, defined as elimination. Disposition is therefore a combination distribution and elimination.

\section{Distribution}

Once in the systemic circulation, the blood or plasma concentrations of a drug will depend on how extensively it is distributed to extravascular sites [2]. Drug concentration in whole blood represents the total concentrations of drug in the circulatory system. Plasma concentration do not account for drug molecules that are sequestered into red or white blood cells. In general, 
the blood and the plasma concentrations are assumed to be equal unless the drug is preferentially sequestered by red blood cells. Drug distribution will be influenced by tissue/organ blood flow, whether the drug is able to passively diffuse across cell membranes or is a substrate for active uptake or efflux transporters, and its extent of binding to plasma protein and tissue sites.

\subsection{Tissue/organ blood flow}

The transfer of many drug compounds from the systemic circulation to various tissues/organs follows the perfusion-rate diffusion process. Here, we assume that cell membranes do not present any barrier to drug transfer. This typically applies to drug compounds that are lipid soluble. Under perfusion-rate diffusion, the rate of delivery from the systemic circulation to a specific tissue/organ is primarily dependent on the blood flow within an organ or tissue. Organs like the liver and the heart are highly perfused with blood. By contrast, the bone and the adipose tissues experience less blood perfusion. Therefore, drugs are likely to distribute more rapidly to tissues/organs that are more richly perfused with blood.

\subsection{Passive diffusion across cell membranes}

A major factor affecting drug distribution is the physicochemical properties of the drug [3] since these would influence the permeability of the drug to various tissues. A drug that is highly lipophilic, such as chloroquine, may readily cross the lipidic bilayer of endothelial cells and most cell membranes to reach into the intracellular space via passive transcellular diffusion. Lipid-soluble drugs, because of their high partition coefficient, can also accumulate in organs or sites with fat deposits. On the other hand, drugs that are more water soluble and polar, such as aminoglycosides, do not distribute well into most tissues/organs. For such drug molecules, entry into the tissue spaces may rely on either paracellular diffusion via gaps inbetween cells [4] or carrier-mediated uptake transport processes.

\subsection{Influx or efflux transporters}

Influx and efflux transporter are found in many tissues/organs and play a role in the distribution of drugs in the body [5]. The efflux transporter, P-glycoprotein (P-gp), which is expressed in the liver and the kidney, functions to keep drugs out of tissues [6]. By contrast, the influx transporter OATP1B1, an organic anion transporter expressed in the liver and the brain, acts on drug substrates to move them from the extracellular matrix into the tissue spaces. Since these transporters are subject to genetic polymorphisms, their underexpression or overexpression will result in differences in the extent of drug distribution between patients.

\subsection{Plasma protein and tissue binding}

Another factor influencing drug distribution is the preferential binding to plasma proteins and tissues [7]. It is the unbound or free portion of the drug that diffuses out of the plasma into the tissues/organs. Albumin and $\alpha_{1}$-acid glycoprotein are the two major proteins in plasma that are responsible for the binding of most drug compounds in the systemic circulation. The extent of plasma protein binding of a drug can be drug- or protein-concentration dependent, based 
on the affinity and capacity of the plasma protein. A drug's protein-binding characteristics also depend on its physicochemical properties, with lipophilic drugs more likely to bind to plasma proteins and consequently, less available to the intracellular spaces [8]. Table 1 lists the extent of plasma protein binding of selected drugs. Binding to tissues also affects drug concentrations in the blood/plasma and the tissues/organs. However, compared to plasma protein binding, much less is known about tissue binding or the sequestration of drugs, since reliable methods for estimating binding to tissue components in vivo are experimentally more challenging.

\begin{tabular}{ll}
\hline Drug & Binding \\
\hline Gentamicin & 3 \\
\hline Digoxin & 25 \\
\hline Vancomycin & 30 \\
\hline Lidocaine & 51 \\
\hline Phenytoin & 89 \\
\hline Warfarin & 99 \\
\hline
\end{tabular}

Table 1. Extent of plasma protein binding of selected drugs.

\section{Volume of distribution}

A quantitative analysis of distribution is needed to understand pharmacokinetics of a drug. A drug can be characterised by the volume of fluids into which it distributes [9]. Since the volume of body fluids cannot be easily measured, it is assumed that the body simplifies into a tank of fluid into which the drug is placed. This volume is known as $<\mathrm{s} \$ \% \&$ ? >the volume of distribution $\left(V_{d}\right)$. The $V_{d}$ of a drug is an important pharmacokinetic parameter and is defined as the ratio of the amount of drug in the body to the concentration in a biological matrix that is readily accessible, such as the plasma. The $V_{d}$ has units of volume, such as litre.

The $V_{d}$ of a drug given as an intravenous (i.v.) dose can be calculated by:

$$
V_{d}=\frac{A}{C_{p}}
$$

where $A$ and $C_{p}$ are the i.v. dose of the drug (units: mass) and the drug concentration (units, e.g.: $\mathrm{g} / \mathrm{L}$ ) in plasma at time zero, respectively. If direct measurement is impractical, this initial drug concentration is derived from the $y$-axis intercept of the extrapolated logarithmic concentration versus time line. In general, it is assumed that $C_{p}$ is the total drug concentration of the drug (free and bound to plasma proteins). 
The $V_{d}$ of a drug is regarded as a hypothetical term as it has no direct correlation to anatomical spaces in the body. It denotes the apparent volume of space into which a drug can distribute after dosing, and is indicative of its relative storage in the plasma and in the tissue/organ spaces. For this reason, $V_{d}$ has also been commonly called the apparent volume of distribution. The $V_{d}$ provides an important guide when accessing the tissue penetration of a drug. Table 2 lists the $V_{d}$ of selected drugs.

\begin{tabular}{ll}
\hline Drug & $\mathbf{V}_{\mathbf{d}}(\mathbf{L})$ \\
\hline Warfarin & 8 \\
\hline Gentamicin & 18 \\
\hline Digoxin & 440 \\
\hline Diazepam & 80 \\
\hline Nortriptyline & 1700 \\
\hline Chloroquine & $>15000$ \\
\hline
\end{tabular}

Table 2. The $V_{d}$ of selected drugs.

The concept of $V_{d}$ is illustrated in Figure 1.

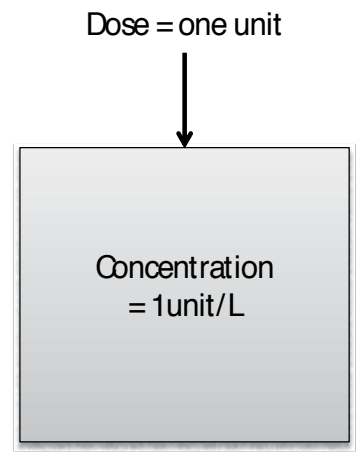

Container 1

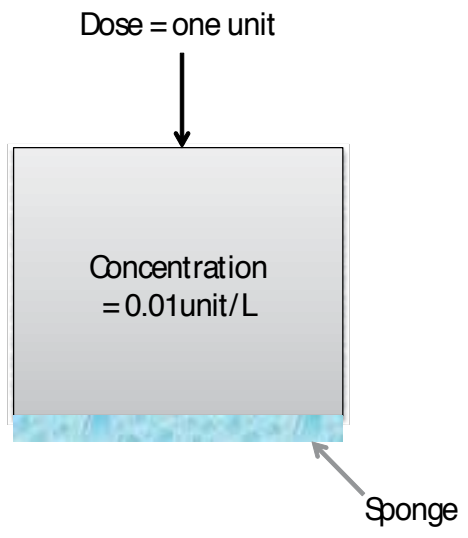

Container 2

Figure 1. A clarification of the concept of $V_{d}$ of a drug dissolved in two containers containing water. The $V_{d}$ is an apparent volume term that is determined from the amount of drug added and the resulting concentration. Given the same dose amount and the 100-fold reduction in drug concentration in Container 2, the $V_{d}$ of the drug in Container 2 is 100 times larger than that of the same drug present in Container 1.

Let us imagine there are two containers of the same size filled with $1 \mathrm{~L}$ of water: Container 1 and Container 2. Container 2 also contains a small quantity of sponge that adds no significant volume to the overall container volume. A one unit dose of the same drug is then added to 
each container. After complete dissolution of the drug in the volume of water within each container, the drug concentrations are measured. The concentrations in Container 1 and Container 2 are found to be 1unit/L and 0.01unit/L, respectively. Here, it is assumed that each container is a closed system after drug intake: no drug elimination occurs during the time for complete drug dissolution. By dividing the dose (one unit) by the measured concentration in each container (Eq. (1)), the volumes derived are 1L and 100L for Container 1 and Container 2 , respectively. The explanation for this observation is that some of the drug in Container 2 is bound to the sponge, which renders it unavailable for measurement of the drug concentration in water. Consequently, the measured drug concentration in Container 2 is low and the calculated volume is high. However, each container still contains one unit of drug since none is eliminated. Put in another way, the $V_{d}$ is an apparent or hypothetical quantity that relates the total drug amount in the system (dose) to its concentration in a matrix of measurement (water).

A physiology-driven formula has been proposed that accounts for the influence of blood or plasma volume, tissue volume (difference between total body water volume and plasma volume), drug binding to plasma proteins and drug binding to tissue sites on the $V_{d}$ of a specific drug [10]. This formula is given by the following equation:

$$
V_{d}=V_{p}+V_{t} \times \frac{f_{u, p}}{f_{u, t}}
$$

where $V_{u, p}, V_{u, t} f_{u}$ and $f_{t}$ denote the plasma volume, tissue volume, fraction of the drug unbound in plasma, and fraction of the drug unbound in tissue, respectively. Drugs such as nortriptyline and chloroquine have a large distribution volume, which indicates significant uptake and binding to tissue binding sites. By contrast, large-sized drug compounds (e.g. heparin), drugs that preferentially bind to plasma proteins (e.g. warfarin) and monoclonal antibodies, have a small $V_{d}$ indicating that these remain mainly in the vascular space.

\section{Elimination}

Effective drug therapy involves achieving optimal efficacy without causing toxicity [11]. To this end, drug intake into and distribution within the body must be balanced with elimination so that appropriate concentrations at the receptor sites can be achieved. Elimination refers to the irreversible removal of a drug or its metabolite(s) from the body. For the majority of drugs, metabolism is the major pathway of elimination $[12,13]$. The primary organ involved is the liver, although the gastrointestinal (GI) tract, kidney, lung and skin may also contain drug metabolising enzymes and may contribute to regional concentrations of the drug and the metabolites. Excretion of drugs and their metabolites mainly occur in the kidneys, but may also involve the GI tract and lung. This section describes the key aspects of liver metabolism and factors that may govern it, and the components of renal and biliary excretion. 


\subsection{Liver metabolism}

In the liver, a wide array of enzymes exists to biotransform drugs, producing less active (or in some cases more active) metabolites [14]. Drug metabolism is defined as the biotransformation of lipid-soluble chemicals into water-soluble forms, so that these can be excreted in the urine. Metabolism is divided into two phases (Figure 2). Drugs may undergo one phase only, or be metabolised through both phases sequentially.

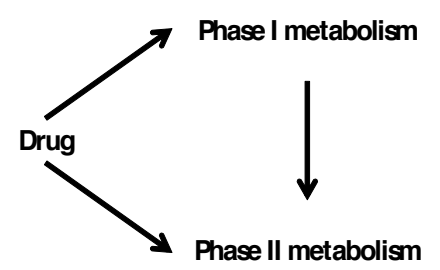

Figure 2. The phases of drug metabolism. Phase I reaction functionalises the drug with a 'reactive' group. Phase II metabolism typically results from the conjugation of an endogenous molecule to the 'reactive' group.

\subsubsection{Phase I metabolism}

Phase I reactions involve the introduction into or unveiling of a polar functional group (e.g. $\mathrm{OH},-\mathrm{SH}$ ) on the drug molecule, rendering it a suitable substrate for conjugation with another molecule during phase II metabolism. Such reactions typically involve oxidation, reduction or hydrolysis processes. Often, the by-product of phase I metabolism, called a derivative, is pharmacologically inactive but more chemically reactive than the parent drug, and may be toxic or even carcinogenic.

The major liver enzyme system involved in phase I metabolism (oxidation) is the cytochrome P450 (CYP) enzyme system [15]. Thus far, 18 CYP families have been identified in mammals, although only CYP1, CYP2, CYP3 and CYP4 are involved in drug metabolism, with CYP1A2, CYP2C9, CYP2C19, CYP2D6, CYP2E1 and CYP3A4 being responsible for the biotransformation of greater than $90 \%$ of drugs undergoing phase I metabolism (Table 3).

\begin{tabular}{llll}
\hline CYP enzyme & Examples of substrate drugs & Induced by & Inhibited by \\
\hline CYP1A2 & $\begin{array}{l}\text { Acetaminophen, caffeine, } \\
\text { theophylline }\end{array}$ & Smoking & Ciprofloxacin, fluvoxamine \\
\hline CYP2C9 & Warfarin, phenytoin & Phenytoin, carbamazepine & Fluoxetine \\
\hline CYP2C19 & Omeprazole, phenytoin & Phenytoin, carbamazepine & Fluvoxamine \\
\hline CYP2D6 & Codeine, risperidone & Glutethimide & Fluoxetine \\
\hline CYP2E1 & Acetaminophen, ethanol & Ethanol & Disulfram \\
\hline CYP3A4 & Midazolam, simvastatin & Rifampicin & Ritonavir, ketoconazole \\
\hline
\end{tabular}

Table 3. Selected CYP enzyme-substrate drugs and their respective inducers and inhibitors. 
In addition to genetic polymorphism, liver CYP enzymes are subject to induction and inhibition by certain drugs. As a consequence, elimination of such CYP enzyme-substrate drugs administered concomitantly may increase or decrease. Additionally, where metabolic pathways involve the production of pharmacologically active or toxic metabolites, induction or inhibition of CYP enzymes could result in unanticipated changes in plasma drug concentrations, with potential clinical relevance to its therapeutic or toxicity profile. Some examples of inducers or inhibitors of CYP enzymes are provided in Table 3.

\subsubsection{Phase II metabolism}

The derivative from phase I metabolism may be excreted via the urine immediately if high aqueous solubility is achieved. If not, the derivative undergoes a phase II reaction that brings about the conjugation of its functional group(s) to various hydrophilic endogenous compounds [16]. Examples of phase II reactions include sulfation, glucuronidation and glutathione conjugation. Sufficient water solubility is normally achieved in conjugates, which facilitate renal excretion. In addition, the insertion of a large polar substrate to the parent drug or derivative would make it more amenable for active secretion into the bile for subsequent excretion into the GI tract.

\subsubsection{Prodrugs}

After a phase I metabolism reaction, a drug may become "activated" or pharmacologically active. This biotransformation process is the basis for the development and usage of prodrugs [17]. The prodrug is typically a structural derivative of the active drug and synthesised by adding or changing a functional group(s) on the active drug structure. The ester is a common prodrug form of drug with hydroxyl or carboxylic groups. Esters can be synthesised with desired degrees of lipophilicity or hydrophilicity, and with controlled rates of the activating hydrolytic reaction. Once the prodrug gets inside the body, enzymes work to metabolically cleave the prodrug in order to form the active drug. Examples of prodrugs include levodopa, which is an amino acid derivative form of dopamine, and codeine, which is metabolised in the body to form morphine for analgesic effect.

There are many reasons to administer a prodrug in lieu of the active drug. The active drug may be too polar or hydrophilic for sufficient absorption and oral bioavailability to be attained, or for transfer across into the lipidic cell membranes to reach receptor sites, such as in neurons. Under such conditions, a functional group, such as carboxylic or hydroxyl group, may be attached to the active drug in order to enhance membrane transport. After absorption and distribution to the site of action, the functional group is cleaved via metabolism to release the active drug. In this regard, esterases found in almost all tissues make conversion of prodrugs into active drugs relatively straightforward. Other reasons for synthesising prodrugs are poor stability or poor patient acceptability (odour, pain on injection, gastric irritation) of the active drug, or a need to prolong the stay of the drug in the body. 


\subsection{Drug excretion}

Excretion is the principal mode of termination of drug and metabolite effects. Drugs and their metabolite(s) are most commonly removed from the body via two main routes: renal and biliary excretion.

\subsubsection{Renal excretion}

About $25 \%$ of cardiac output goes to the kidney at which a significant portion of foreign compounds are filtered out. Renal excretion incorporates the processes of glomerular filtration, reabsorption from the renal tubular lumen, and tubular secretion as the drug passes through the nephron, the functional excretory unit of the kidney [18].

As blood passes through the glomerulus, entities within it are filtered to form the renal filtrate in the tubular lumen. The process of filtration is passive in nature and is driven by a combination of the large hydrostatic and concentration gradients present across the glomerulusBowman's capsule junction. Nevertheless, large-sized components cannot be filtered through the glomerular membrane, which implies that large drugs (e.g. heparin), plasma proteins and plasma protein-bound drugs (e.g. warfarin) cannot cross into the tubular filtrate.

Water is reabsorbed along the nephron tubule so that only $1 \%$ of the original filtrate is passed out of the body as urine. Approximately $99 \%$ of substances filtered at the glomerulus are reabsorbed along the renal tubules. The majority of filtered, unmetabolised drug molecules are also reabsorbed, especially if these are lipophilic. This is because such drugs are more likely to cross the membranes of the cells lining the tubules. By contrast, polar drugs e.g. gentamicin and digoxin, are unable to do this. Such drugs will therefore be excreted unchanged in the urine because they do not need to undergo biotransformation to increase their water solubility.

Active secretion into the renal tubules occurs for some drugs that are not readily filtered in the glomerulus. This pathway occurs via a carrier mechanism and is sufficiently efficient as to not depend on binding between plasma proteins and drugs, ensuring almost complete clearance of drugs such as penicillin [19]. Other drugs excreted by this process include anti-inflammatory drugs and methotrexate.

There may be competition for the active transport sites amongst drug compounds [20]. This may be exploited for therapeutic care, such as probenecid inhibiting the active secretion of penicillin from the kidney, increasing the latter's elimination half-life and prolonging its effect on the body. Conversely, competition for transport sites may lead to increased morbidity, e.g. aspirin can inhibit the secretion of uric acid, leading to gout aggravation.

Renal clearance of a drug can be measured with timed collection of urine and analysis of the drug concentration in the urine using the following equation [21]:

$$
C L_{R}=\frac{C_{u} \times Q_{u}}{C_{p}}
$$


where $C L_{R}$ is renal clearance, $C_{u}$ is the concentration of the drug in urine, $Q_{u}$ is the volume of urine formation per unit time, and $C_{p}$ is the concentration of the drug in plasma. Here, $C_{u} \times Q_{u}$ represents the excretion rate of drug in urine.

\subsubsection{Biliary excretion}

While in the liver, drugs or metabolites can also be secreted into the bile in much the same manner as the kidney secretes drugs into the nephron tubular filtrate [22, 23]. Biliary excretion is facilitated by active transport systems located in the canalicular membrane of the hepatocyte, and can be an important hepatic elimination pathway for many compounds. Since bile is an aqueous solution, it is suitable for dissolving hydrophilic drugs. In addition, bile acids allow solubilisation of lipid-soluble drugs. Thus, all types of species (anionic, cationic and un-ionised drugs), polar and lipophilic, can be secreted into the bile. These include drug metabolites that have undergone conjugation with glucuronate during phase II metabolism. The main criterion for significant biliary excretion seems to be molecular weight $>500$.

Once bile and its constituents enter into the intestines, many organic biliary constituents, including bile salts and cholesterol, are reabsorbed from intestines back into the blood with high efficiency. These components then return to the liver via the hepatic portal vein. Drugs or metabolites excreted in the bile may recirculate in the same manner. If the drug has favourable physicochemical properties, it can be partially reabsorbed from the intestines back into the blood stream just like an orally ingested drug. Metabolites with glucuronate or sulfate groups may be removed by enzymes produced by the resident bacteria of the lower small intestine and colon, and the now-active drug is able to be reabsorbed. One example is mycophenolic acid. This immunosuppressant drug undergoes conjugation to glucuronate in the liver. The glucuronide metabolite of mycophenolic acid is secreted into the bile, cleaved in the small intestines, and reabsorbed back into the systemic circulation as the parent drug compound. Thus, a reservoir of the drug is established in the enterohepatic circulation, with an ongoing cycle of absorption, metabolism, secretion into the bile and reabsorption. Enterohepatic circulation hence increases the persistence of drugs in the body, and reduces overall clearance in the bile.

The clearance of a drug from various eliminating tissues occurs in parallel so the total body clearance of the drug $\left(C L_{T}\right)$ is equal to the sum of the clearances of the individual tissues:

$$
C L_{T}=C L_{R}+C L_{H}+C L_{L}+C L_{\text {other }}
$$

where $C L_{H}$ is hepatic clearance of the drug, $C L_{L}$ is clearance of the drug from the lung, and $C L_{\text {other }}$ denotes the respective clearance values from the other eliminating tissues.

Clearance is a constant that describes the relationship between drug concentration $(C(t))$ in the body and the rate of elimination of the drug from the body and has units of volume per time.

Figure 3 shows a way of schematically visualising $C L$. 

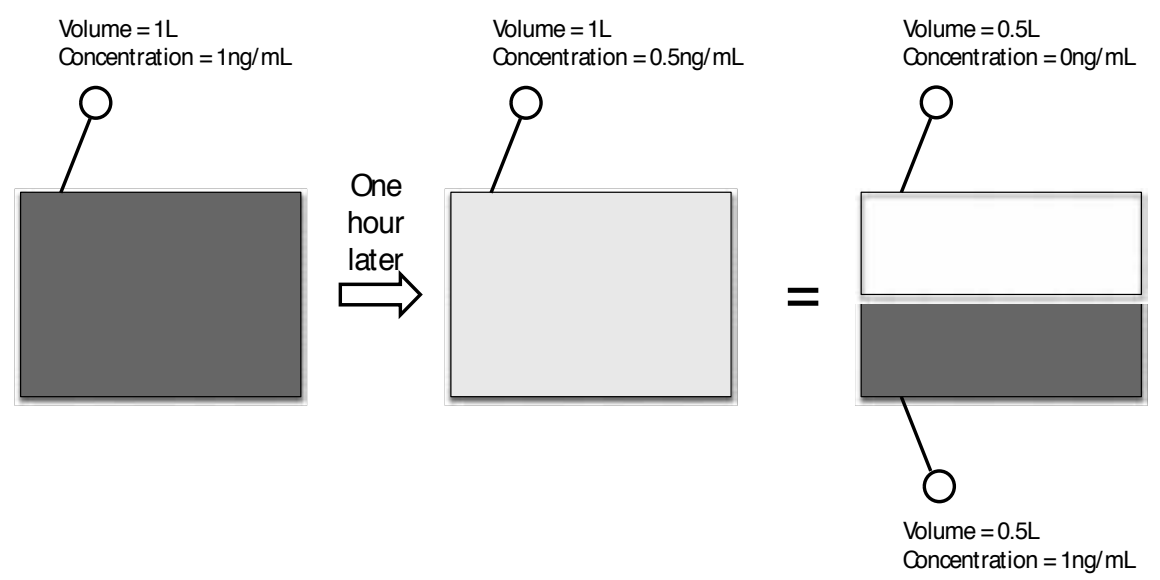

Figure 3. Clearance may be viewed as the volume of plasma from which the drug is totally removed over a specified time period.

Here, it is assumed that a container is present that contains $1 \mathrm{~L}$ of water with a drug concentration of $1 \mathrm{ng} / \mathrm{mL}$ immediately after drug intake (Figure 3, left container). The container is used to denote the human body (the system) and water is used to denote the plasma. After one hour, the drug concentration is measured in the $1 \mathrm{~L}$ of water and found to decrease to $0.5 \mathrm{ng} / \mathrm{mL}$ due to drug elimination (Figure 3, middle container). Another way of viewing this is that, after one hour, half of the water volume contains the original drug concentration $(1 \mathrm{ng} / \mathrm{mL})$, whereas the other half of the water volume is completely voided of the drug (Figure 3, right container). This means that the clearance of the drug is $500 \mathrm{~mL} / \mathrm{h}$.

Clearance can also be understood as the product of the perfusion of the eliminating organ $(\mathrm{Q})$ and the intrinsic ability of the organ to eliminate the drug termed extraction (E):

$$
C L=Q \times E
$$

where $E$ is calculated by $\frac{C_{\text {in }}-C_{\text {out }}}{C_{\text {in }}}$, and $C_{\text {in }}$ and $C_{\text {out }}$ are the drug concentrations in the blood entering and leaving the organ, respectively. Since $E$ is unitless, $C L$ has the same units as perfusion (volume per time). The $C L$ of the drug is always constant and is considered a primary parameter. This is a physiologically appealing definition of $C L$ in the sense that alterations in perfusion and extraction can be shown to change $C L$ in a predictable manner.

\section{Introduction to compartmental modelling}

The body can be regarded as compartmental systems to describe the many processes involved in the absorption, distribution, metabolism and excretion of drugs. Three different approaches can be used to describe the pharmacokinetics of drugs in the body: 
1. Non-compartmental (NCA) pharmacokinetic model

2. Compartmental pharmacokinetic model

3. Physiologically based pharmacokinetic (PBPK) model

The NCA approach (using statistical moment analysis) does not require the assumption of any compartments for the purpose of data analysis [24]. This method is based on the area under the drug concentration versus time curve (AUC) and the mean residence time (MRT). Although the NCA approach can be applied to most pharmacokinetic data, it lacks the ability to predict pharmacokinetic profiles when there are changes to the dosing regimen since it cannot estimate the concentration value at a specific time point.

The compartmental approach divides the body into a series of pharmacokinetically distinct compartments, each of which denotes a collection of tissues and organs that have similar rate of change of the drug concentration [25]. Drugs may exhibit single- or multi-compartment plasma concentration versus time profiles, with the number of compartments referring to the total number of disposition compartments. The compartment model assumes that each of the compartments is a well-stirred, kinetically homogenous unit. In addition, it is often assumed when constructing a compartmental model that the rate of elimination of the drug from the compartment and the transfer of the drug between the compartments (for a multi-compartment model) follows first-order (linear) kinetics. Under first-order kinetics, the rate of change of the drug amount or concentration is directly proportional to the remaining drug amount or concentration within the compartment.

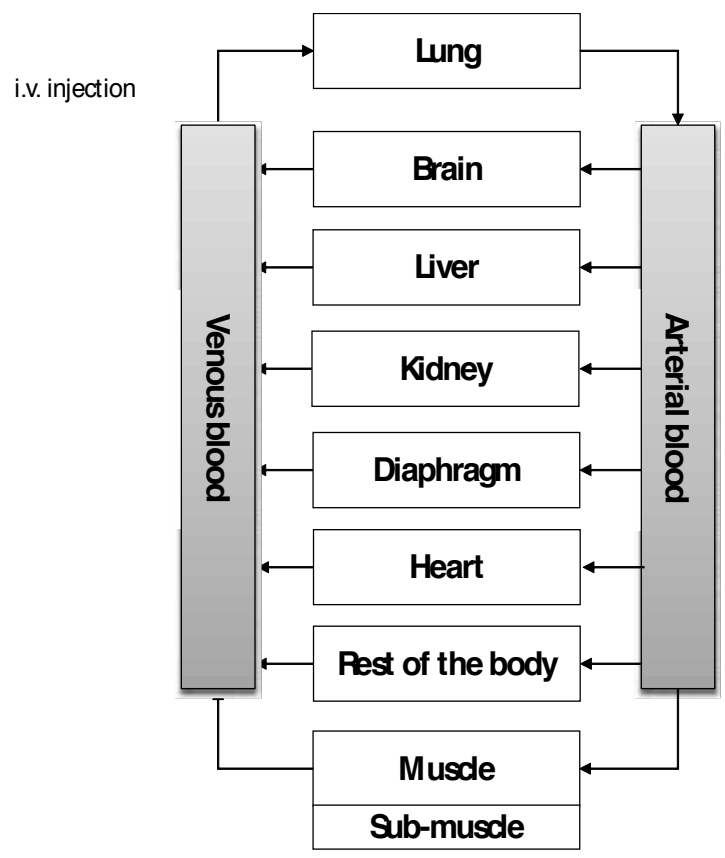

Figure 4. A physiologically based pharmacokinetic (PBPK) model for the description of drug pharmacokinetics in anatomically relevant tissues and organs. Here, the drug is administered as a bolus dose into the venous blood compartment. 
Unlike the aforementioned compartment model, a PBPK model (Figure 4) comprises compartments that are defined based on anatomy, e.g. a compartment each for the liver and the brain. In addition, the PBPK model is also based on actual physiological and biochemical factors important in the input and disposition of the drug [26]. Such factors include cardiac output, organ blood flow rates, blood-to-plasma drug concentration ratio, partition coefficients in each organ, and transporter activities.

\subsection{One-compartment model}

Although more complex pharmacokinetic models may be necessary, a one-compartment model with first-order input provides a reasonable description of the time course for many drugs given at therapeutic doses (Figure 5), for example [27-29].

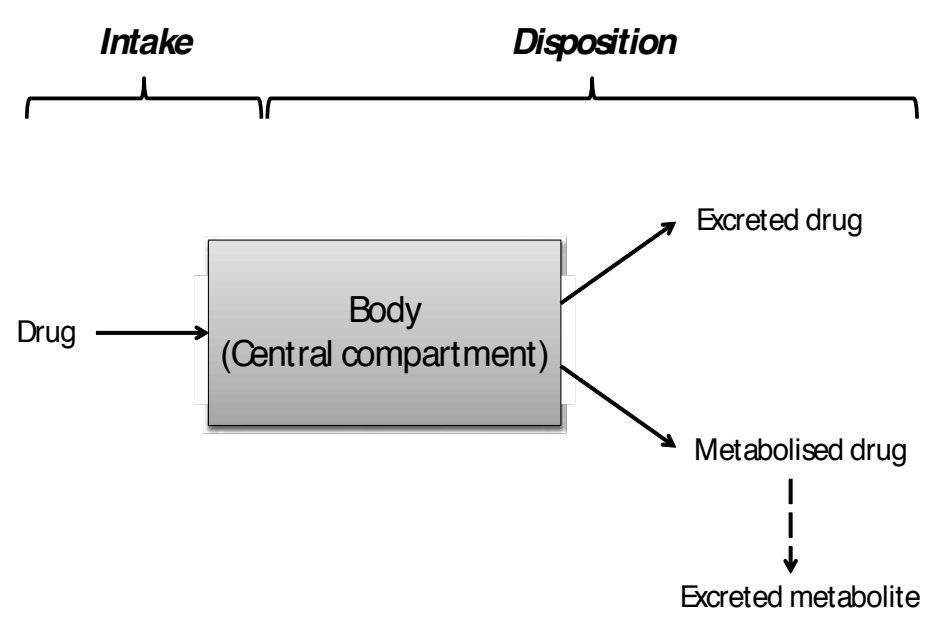

Figure 5. A one-compartment model with first-order input for describing the drug plasma concentration versus time profile. The arrows indicate the movement of the drug with respect to the body. Further, the diagram depicts that part of the drug may be excreted unchanged and some part may be metabolised by enzymes in the body.

In the one-compartment model, all the tissues of the body are lumped together as a single kinetically homogeneous compartment, commonly referred to as the central compartment. The one-compartment model is typically applied to drugs that distribute to only richlyperfused tissues and organs, such as liver, kidney and brain, in addition to the systemic circulation. The strongest indication that the body behaves as a single pharmacokinetically homogenous compartment for a drug is given by the presence of a mono-exponential decline in concentration values with respect to time when the concentrations are plotted on a logarithmic scale (Figure 6). For such a drug, it is assumed that all tissues and organs have a similar rate of change of the drug concentration as that of the systemic circulation, i.e. the source compartment. 

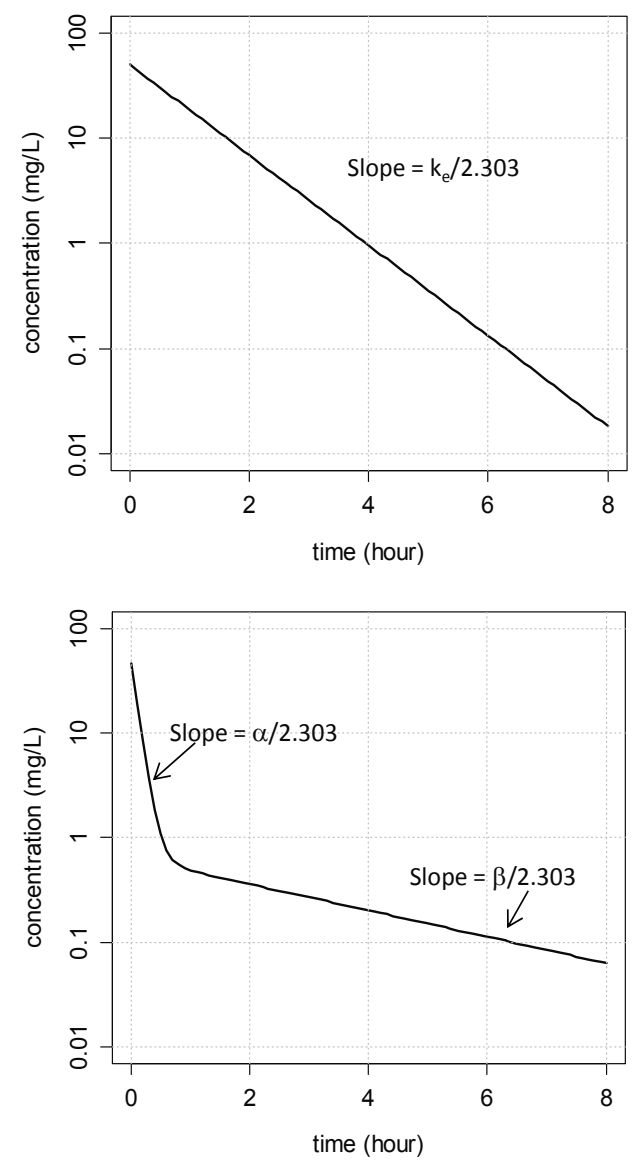

Figure 6. Plasma concentration versus time profiles following i.v. bolus administration on a semi-logarithmic graph for a drug exhibiting a one-compartment model (upper panel) and a two-compartment model (lower panel). For the twocompartment model, the slopes $\alpha$ and $\beta$ denote the rates of combined distribution plus elimination, and elimination, respectively, of the drug from the central compartment.

After intravenous (i.v.) bolus administration, the blood or plasma drug concentrations $(C(t))$ at time $t$ may be represented as:

$$
C(t)=\frac{\text { Dose }_{i v}}{V_{d}} \times e^{-k_{e} \times t}
$$

where $\operatorname{Dose}_{i v}, V_{d}$ and $k_{e}$ are the administered i.v. dose (units: mass), volume of distribution (units: volume), and first-order elimination rate constant (units: reciprocal time), respectively. This equation may be converted to the natural logarithm to yield:

$$
\ln C(t)=\ln \left(\frac{\operatorname{Dose}_{i v}}{V_{d}}\right)-k_{e} \times t
$$


The total body clearance $\left(C L_{T}\right)$ and elimination half-life, $t_{1 / 2}$ (units: time), can be calculated by:

$$
\begin{gathered}
C L_{T}=k_{e} \times V_{d} \\
t_{1 / 2}=\frac{0.693}{k_{e}}
\end{gathered}
$$

For an i.v. administered drug displaying one-compartmental pharmacokinetic behaviour, drug concentrations of tissues will decay in parallel with plasma concentrations. However, it does not imply that the concentration in the plasma is equal to the concentration in these body tissues.

When a drug is administered extravascularly, such as oral ingestion, it has to be absorbed through biological barriers prior to reaching the central compartment (blood or plasma). Only when the drug enters the blood or plasma will it be regarded as systemically available. The process of absorption is complex and is governed by myriad factors including the administration route, formulation type, dose amount, and the physicochemical properties of the drug. Following extravascular administration, compartment models become more complicated because now the drug's absorption rate constant $\left(k_{a}\right)$ needs to be considered in the model. Despite the complexity involved, drug absorption is generally regarded as a first-order input process. For this first-order input, one-compartment model, the drug concentration at any time $t$ is given by:

$$
C(t)=\frac{F \times k_{a} \times \text { Dose }_{e x}}{V_{d} \times\left(k_{a}-k_{e}\right)} \times\left(e^{-k_{e} \times t}-e^{-k_{a} \times t}\right)
$$

where $F$ and $D e_{e x}$ are the bioavailability (no units) and extravascular dose (units: mass), respectively. $F$ denotes the proportion of extravascularly-administered drug that reaches the system circulation after drug administration. The maximum concentration $\left(C_{\max }\right)$ after extravascular administration and the time at which $C_{\max }$ is attained $\left(t_{\max }\right)$ are calculated by:

$$
\begin{gathered}
C_{\max }=\frac{F \times \operatorname{Dose}_{e x}}{V_{d}} \times e^{-k_{e} \times t_{\max }} \\
t_{\max }=\frac{\ln \left(k_{a} / k_{e}\right)}{k_{a}-k_{e}}
\end{gathered}
$$

\subsection{Two-compartment model}

Sometimes, drugs may display two or more phases during the declining portion of the concentration versus time profile [30]. This phenomenon may be encountered when multiple 
blood samples are collected during the drug's distribution and elimination phases. When the plasma drug concentration exhibits a bi-exponential decay (Figure 6, lower panel) on a semilogarithmic scale following an i.v. bolus injection, a two-compartment model is necessary to describe the underlying pharmacokinetics. The two-compartment model divides the body into the central compartment as per the one-compartment model, as well as a peripheral compartment that lumps together slowly-perfused tissues, such as fat and muscle. It is generally assumed that drug is eliminated still from the central compartment that comprises the liver and the kidney, since most compounds are metabolised by the liver and/or undergo renal excretion.

As portrayed by Figure 6 (lower panel), the concentration versus time profile after a single i.v. bolus dose on the semi- logarithmic scale has a rapidly declining phase followed by a shallower declining phase. The initial rapid decline in concentration is a consequence of simultaneous elimination (to the external environment) and distribution of the drug from the plasma to tissues lumped under the peripheral compartment. After the distribution process is completed and equilibrium is established between drug concentrations in the central compartment and the peripheral compartment, the drug concentration in the central compartment decreases at a rate dependent on the drug elimination rate. In general, the drug elimination rate is lower than the initial rate of decline in concentration due to the simultaneous distribution and elimination of the drug from the central compartment.

Without specifically describing the micro rate constants, the drug concentration at any time $t$ after a single i.v. bolus administration of a two-compartment system is represented by:

$$
C(t)=A \times e^{-\infty \times t}+B \times e^{-\beta \times t}
$$

where $A, B, \alpha$ and $\beta$ are derived from the intercepts and slopes of the respective distribution plus elimination, and elimination phases of the drug concentration versus time profile by curve fitting, such as nonlinear regression analysis.

\section{Conclusion}

Drug disposition refers to the combination of distribution and elimination. Distribution is a reversible process of movement of drugs from and to the site of measurement, typically the plasma or blood. Elimination comprises metabolism and excretion, and represents the total irreversible loss of the drug from the body. Drug disposition and elimination contribute to overall efficacy or toxicity and hence, up-to-date understanding of these pharmacokinetic processes is essential to safe, professional practice around medications and drug treatment. Compartmental modelling is a useful method to understand distribution and excretion processes [27, 31]. 


\section{Author details}

Seng Kok-Yong ${ }^{1}$ and Lee Lawrence ${ }^{2^{*}}$

*Address all correspondence to: lawrence_lee@nuhs.edu.sg

1 Defence Medical \& Environmental Research Institute, DSO National Laboratories, Singapore, Singapore

2 Department of Medicine, National University Health System, Singapore, Singapore

\section{References}

[1] Dale MM, Rang HP. Rang and Dale's Pharmacology, 7th ed. New York: Elsevier/ Churchill Livingstone, 2012, pp. 123-131.

[2] Urso R, Blardi P, Giorgi G. A short introduction to pharmacokinetics. European Review for Medical and Pharmacological Sciences. 2002;6(2-3):33-44.

[3] Leeson P. Drug discovery: Chemical beauty contest. Nature. 2012;481(7382):455-456. doi: $10.1038 / 481455 a$.

[4] Madara JL. Regulation of the movement of solutes across tight junctions. Annual Review of Physiology. 1998;60:143-159. doi: 10.1146/annurev.physiol.60.1.143

[5] Grover A, Benet LZ. Effects of drug transporters on volume of distribution. The AAPS Journal. 2009;11(2):250-261. doi: 10.1208/s12248-009-9102-7

[6] Chiou WL, Chung SM, Wu TC. Potential role of P-glycoprotein in affecting hepatic metabolism of drugs. Pharmaceutical Research. 2000;17(8):903-905.

[7] Schmidt S, Gonzalez D, Derendorf H. Significance of protein binding in pharmacokinetics and pharmacodynamics. Journal of Pharmaceutical Sciences. 2010;99(3):11071122. doi: 10.1002/jps.21916

[8] Lin JH, Lu AY. Role of pharmacokinetics and metabolism in drug discovery and development. Pharmacological Reviews. 1997;49(4):403-449.

[9] Smith BE. Pharmacokinetics without calculus - an introduction. International Anesthesiology Clinics. 1995;33(3):11-28.

[10] Wilkinson GR, Shand DG. Commentary: a physiological approach to hepatic drug clearance. Clinical Pharmacology and Therapeutics. 1975;18(4):377-390.

[11] Abdel-Rahman SM, Kauffman RE. The integration of pharmacokinetics and pharmacodynamics: understanding dose-response. Annual Review of Pharmacology and Toxicology. 2004;44:111-136. doi: 10.1146/annurev.pharmtox.44.101802.121347 
[12] Wienkers LC, Heath TG. Predicting in vivo drug interactions from in vitro drug discovery data. Nature Reviews Drug Discovery. 2005;4(10):825-833. doi: 10.1038/ nrd1851

[13] Williams JA, Hyland R, Jones BC, Smith DA, Hurst S, Goosen TC, et al. Drug-drug interactions for UDP-glucuronosyltransferase substrates: a pharmacokinetic explanation for typically observed low exposure (AUCi/AUC) ratios. Drug Metabolism and Disposition: The Biological Fate of Chemicals. 2004;32(11):1201-1208. doi: 10.1124/ dmd.104.000794

[14] Pandit NK, Soltis R. Introduction to the Pharmaceutical Sciences: In Integrated Approach, 2nd ed. Baltimore: Wolters Kluwer/Lippincott Williams \& Wilkins, 2012.

[15] Lynch T, Price A. The effect of cytochrome P450 metabolism on drug response, interactions, and adverse effects. American Family Physician. 2007;76(3):391-396.

[16] Rubin GM, Tozer TN. Hepatic binding and Michaelis-Menten metabolism of drugs. Journal of Pharmaceutical Sciences. 1986;75(7):660-663.

[17] Rautio J, Kumpulainen H, Heimbach T, Oliyai R, Oh D, Jarvinen T, et al. Prodrugs: design and clinical applications. Nature Reviews Drug Discovery. 2008;7(3):255-270. doi: $10.1038 / \mathrm{nrd} 2468$

[18] Rennick BR. Renal excretion of drugs: tubular transport and metabolism. Annual Review of Pharmacology. 1972;12:141-156. doi: 10.1146/annurev.pa.12.040172.001041

[19] Launay-Vacher V, Izzedine H, Karie S, Hulot JS, Baumelou A, Deray G. Renal tubular drug transporters. Nephron Physiology. 2006;103(3):97-106. doi: $10.1159 / 000092212$

[20] Feng B, LaPerle JL, Chang G, Varma MV. Renal clearance in drug discovery and development: molecular descriptors, drug transporters and disease state. Expert Opinion on Drug Metabolism \& Toxicology. 2010;6(8):939-952. doi: $10.1517 / 17425255.2010 .482930$

[21] Tucker GT. Measurement of the renal clearance of drugs. British Journal of Clinical Pharmacology. 1981;12(6):761-770.

[22] Ghibellini G, Leslie EM, Brouwer KL. Methods to evaluate biliary excretion of drugs in humans: an updated review. Molecular Pharmaceutics. 2006;3(3):198-211. doi: 10.1021/mp060011k

[23] Besseghir K, Roch-Ramel F. Renal excretion of drugs and other xenobiotics. Renal Physiology. 1987;10(5):221-241.

[24] Gillespie WR. Noncompartmental versus compartmental modelling in clinical pharmacokinetics. Clinical Pharmacokinetics. 1991;20(4):253-262. doi: 10.2165/00003088-199120040-00001 
[25] DiStefano JJ, 3rd, Landaw EM. Multiexponential, multicompartmental, and noncompartmental modeling. I. Methodological limitations and physiological interpretations. The American Journal of Physiology. 1984;246(5 Pt 2):R651-R664.

[26] Nestorov I. Whole body pharmacokinetic models. Clinical Pharmacokinetics. 2003;42(10):883-908. doi: 10.2165/00003088-200342100-00002

[27] Wang L, Soon GH, Seng KY, Li J, Lee E, Yong EL, et al. Pharmacokinetic modeling of plasma and intracellular concentrations of raltegravir in healthy volunteers. Antimicrobial Agents and Chemotherapy. 2011;55(9):4090-4095. doi: 10.1128/AAC.00593-11

[28] Cooper JM, Duffull SB, Saiao AS, Isbister GK. The pharmacokinetics of sertraline in overdose and the effect of activated charcoal. British Journal of Clinical Pharmacology. 2014. doi: 10.1111/bcp.12500

[29] Wiczling P, Liem RI, Panepinto JA, Garg U, Abdel-Rahman SM, Kearns GL, et al. Population pharmacokinetics of hydroxyurea for children and adolescents with sickle cell disease. Journal of Clinical Pharmacology. 2014;54(9):1016-1022. doi: 10.1002/ jcph.303

[30] Jusko WJ, Gibaldi M. Effects of change in elimination on varous parameters of the two-compartment open model. Journal of Pharmaceutical Sciences. 1972;61(8):1270 1273.

[31] Seng KY, Fan L, Lee HS, Yong WP, Goh BC, Lee LS. Population pharmacokinetics of modafinil and its acid and sulfone metabolites in Chinese males. Therapeutic Drug Monitoring. 2011;33(6):719-729. doi: 10.1097/FTD.0b013e318237a9e9 
Chapter 5

\title{
Biopharmaceutics and Pharmacokinetics
}

\author{
S. Lakshmana Prabu, T.N.K. Suriyaprakash, \\ K. Ruckmani and R. Thirumurugan \\ Additional information is available at the end of the chapter \\ http://dx.doi.org/10.5772/61160
}

\section{Introduction}

Drug research is a specific process toward the development of new therapeutic agents in this era to meet the current medical needs. Drug discovery and development are the two major stages in the development of new therapeutic drug substance. Drug discovery involves identification and characterization of new targets (enzymes or receptors), synthesis of new lead molecules, screening of new lead molecules for its in vitro and/or in vivo biological activities, and physicochemical characterization of leads. The drug discovery and development process requires close interaction among the different scientific discipline members for as many as 10-12 years. It is estimated that only 1 out of 5000 screened compounds is approved as a new drug. On an average, every new drug molecule requires $12 \pm 15$ years to reach the

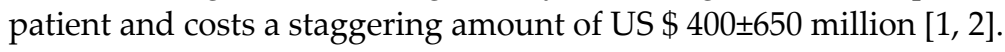

Active pharmaceutical ingredient (API): Any substance or mixture of substances intended to be used in the manufacture of a pharmaceutical dosage form and that, when used so, becomes an active ingredient of that pharmaceutical dosage form [3].

Steps involved in developing a new drug are:

1. Preclinical research

2. Investigational New Drug (IND) Application

3. Phase 1 trials

4. Phase II trials

5. Phase III trials

6. New Drug Application (NDA)

7. Approval [1] 


\section{Human body composition}

Human body is composed of a series of membrane barriers divided by aqueous-filled compartments. These membrane barriers are principally composed of the phospholipid bilayers resulting from the orientation of the lipids (phospholipids, glycolipids, and cholesterol) in the aqueous medium, which surround the cells and also form intracellular barriers around the organelles present in cells (mitochondria, nucleus, etc.). The phospholipids are amphipathic in nature and have aligned polar head groups and lipid "tails," so the polar head groups of phospholipid orientate toward the aqueous phases and the lipid tails form a highly hydrophobic inner core. Hence, the drug substance releases its hydration element and becomes hydrophobic. The drug disposition across the membrane depends on its lipophilicity and partition coefficient. Here, the protein binding plays an important role [4, 5].

The polar molecules will be dissociated in an aqueous environment; thereby, the hydrophilicity arises and vice versa in the case of nonpolar molecules in a lipophilic environment. Every component of an organic compound has a defined lipophilicity. Absorption and bile elimination rate are molecular weight dependent. Lower-molecular-weight compounds have better absorption and less bile excretion when compared to the higher-molecular-weight compounds. Drugs with higher lipophilicity can be better absorbed from the intestine $[5,6]$.

\section{Biopharmaceutics}

Biopharmaceutics is a major branch in pharmaceutical sciences which relates between the physicochemical properties of a drug in dosage form and the pharmacology, toxicology, or clinical response observed after its administration [7]. Drug efficacy and safety are dependent on the dosing regimen. The optimal dosage and dosing intervals can be quite different for different drugs. Moreover, for a single drug, the optimal dosage can be different widely between patients [8].

It is not sufficient to know what the drug does to the body; it is also crucial to know what the body does to the drug. The knowledge of the pharmacodynamic and pharmacokinetic properties of the drug and its metabolites in humans and animals is crucial to understand its different effects among species and for adjusting drug dosing $[9,10]$.

The plasma concentration of the drug is the basic concept of pharmacokinetics. Based on protein binding of the drug, the concentration of free drug available in the circulation influences greatly the dose calculations. The concentration of drug in the plasma is in equilibrium with some tissues in the body [11].

\section{Bioanalytical method}

Blood is the transporter of many vital substances and nutrients for the entire body and thus contains many endogenous and exogenous compounds in different concentrations. Biological 
samples (tissue extracts, plasma, serum, or urine) are extremely complex matrices comprised of many components that can interfere in estimation/quantification; hence, biological samples cannot normally be injected directly into the analyzing system for the determination of active principle. Sample pretreatment is required for achieving sufficient sensitivity and selectivity to determine the active principle. Chemical assays of high quality which include adequate sensitivity, selectivity and reproducibility are essential for obtaining valuable data. Bioanalysis is a subdiscipline of analytical chemistry covering the quantitative measurement drugs and their metabolites in biological systems. Bioanalysis technique can provide a quantitative measure of the active drug and/or its metabolite(s) for the purpose of pharmacokinetics. Various analytical instrument methods such as high-performance liquid chromatography (HPLC) or gas chromatography (GC) or ultra performance liquid chromatography (UPLC) with variety of detectors such as UV, fluorescent, diode array, flame ionization, electron capture and mass spectrometry, and capillary electrophoresis-mass spectrometry may be used. For macromolecule, ELISA or RIA method can be used for quantification $[1,12]$.

\section{Pharmacodynamics}

Pharmacodynamics refers to the relationship between drug concentration at the site of action and the resulting effect, including the time course and intensity of therapeutic and its adverse effects. Studies are designed to investigate all primary and secondary effects related to the desired therapeutic effects, extensions of the therapeutic effect that might produce toxicity at higher doses, and effects related to interactions with other drugs.

\section{Pharmacokinetics}

Pharmacokinetics refers to the study of the time course of a drug within the body (extent and duration of systemic exposure to the drug) and also incorporates the process about the drug's absorption, distribution, metabolism, and excretion (ADME) pattern. In general, pharmacokinetic parameters are derived from the measurement of drug concentrations in blood or plasma [1].

\section{Absorption}

Absorption studies generally involve serial determinations of drug concentration in blood and urine after dosing to indicate the rate and extent of absorption.

Drug absorption refers to the passage of drug molecules from the site of administration into the circulation. Drug absorption requires that drugs cross one or more layers of cells and cell membranes.

Solubility is manipulated mainly by the structure of the drug. In general, solubility is inversely proportional to the number and type of lipophilic functions within the molecule and tightness 
of the crystal packing of the molecule. Solubility decreases when there is increase in crystal packing or lipophilicity.

The concentration of drug in solution is the driving force of the membrane transfer of drug into the body, and low aqueous solubility often continues to present itself as a problem even after formulation improvements.

Factors that influence drug absorption through oral route are:

i. Biological factors: Permeation of the drug across the membrane, GI transit, site specificity, first-pass metabolism, metabolism in the liver, excretion as bile, excretion through bladder, and protein binding of drugs

ii. Pharmaceutical factors: Excipients, type of dosage forms, process of preparation, stability testing, and storage directions

iii. Other factors: Solubility of the drug; partitioning properties; dissociation characteristics; salt formation; particle size, shape, volume, and its distribution; crystallinity; polymorphism; prodrugs; and stereotype and its formation $[8,13,14]$

\section{Drug absorption}

Drugs may be either weak acids or bases that exist in both ionized and non-ionized forms in the body. Drug in the non-ionized form is sufficiently soluble in membrane lipids and can cross cell membranes. The rate of absorption depends upon the ratio of the two forms at a particular site and is also a factor in distribution and elimination. The protonated form of a weak acid is non-ionized, whereas the protonated form of a weak base is ionized. The $\mathrm{pKa}$ is the negative $\log$ of the ionization constant, particular for each acidic or basic drug. Protonated form predominates when the $\mathrm{pH}$ is less than the $\mathrm{pKa}$, whereas nonprotonated form predominates when $\mathrm{pH}$ is greater than the pKa. In the stomach, with a $\mathrm{pH}$ of 1 , weak acids and bases are highly protonated. At this site, the non-ionized form of weak acids $(\mathrm{pKa}=4 \pm 1)$ and the ionized form of weak bases ( $\mathrm{pKa}=9 \pm 1$ ) will prevail upon. Weak acids are absorbed without dissociation than weak base from the stomach and exactly opposite in the intestine where weak bases are absorbed readily than weakly acidic drugs. In intestine, weakly acidic drugs are also found to be absorbed even though they are ionized due to the large surface area [15].

Absorption takes place across the biological membrane by two methods. Lipid drugs are absorbed by transcellular mechanism where the drug distributes into the lipid core of the membrane which diffuses into the other side of the membrane. The solute may also diffuse across the cell membrane and enter into the circulation. Another mechanism is the paracellular absorption. The aqueous-filled pores in between the cells aid absorption of the drugs. Watersoluble drugs are readily absorbed, but the molecule size of the particle plays an important role $[5,12]$.

Drug absorption through transcellular and paracellular pathways is shown in Figure 1. 


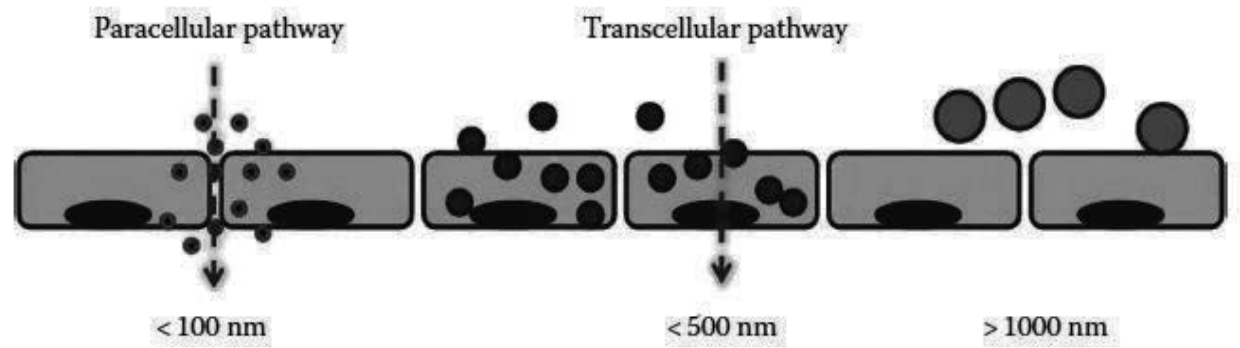

Figure 1. Drug absorption through transcellular and paracellular pathways

\section{Transport across cell membranes}

\subsection{Passive diffusion}

The concentration gradient provides energy for the transportation of the drug across the membrane, and also partitioning of the drug in favor of the lipid membrane decides the quantity of the drug absorbed. The unionized drug is absorbed markedly higher than the ionized form. Passive diffusion could be explained with Fick's first law which relates the diffusive flux to the concentration under the assumption of steady state. It postulates that the flux goes from regions of high concentration to regions of low concentration, with a magnitude that is proportional to the concentration gradient, or in simplistic terms, the concept that a solute will move from a region of high concentration to a region of low concentration across a concentration gradient.

\subsection{Active transport}

Active transport is the movement of molecules across the lipid cell membrane against concentration gradient, i.e., moving from an area of lower concentration in the GIT to an area of higher concentration in the plasma. The absorption sites are at a specific place in the GIT. Active transport is usually associated with accumulating high concentrations of molecules that the cell needs, such as ions, glucose, and amino acids. This active transport process uses chemical energy, such as from adenosine triphosphate (ATP). These energy molecules are site specific - the drugs are transported at a particular site in the GIT, they are limited in number, and they act like a ferry service: it picks a molecule from the GIT, ferries across, leaves in the cytoplasm, and comes back to pick another molecule. The concentration of the drug in the plasma is maintained constant because of this "ferry" service, and the energy/carrier molecules are nothing but ATP-dependent proteins

\subsection{Endocytosis}

Endocytosis is an energy-using process by which cells absorb molecules (such as proteins) by engulfing them. It is used by large polar molecules that cannot pass through the hydrophobic 
plasma or cell membrane. The opposite process is exocytosis. Phagocytosis is a specific form of endocytosis involving the vascular internalization of solids such as bacteria by an organism and is therefore distinct from other forms of endocytosis such as the vesicular internalization of various liquids (pinocytosis). Phagocytosis is involved in the acquisition of nutrients for some cells. Pinocytosis, otherwise known as cell drinking, fluid endocytosis, and bulk-phase pinocytosis, is a mode of endocytosis in which small particles are brought into the cell, forming an invagination and then suspended within small vesicles [14, 16-21]. Various types of endocytosis are shown in Figure 2.

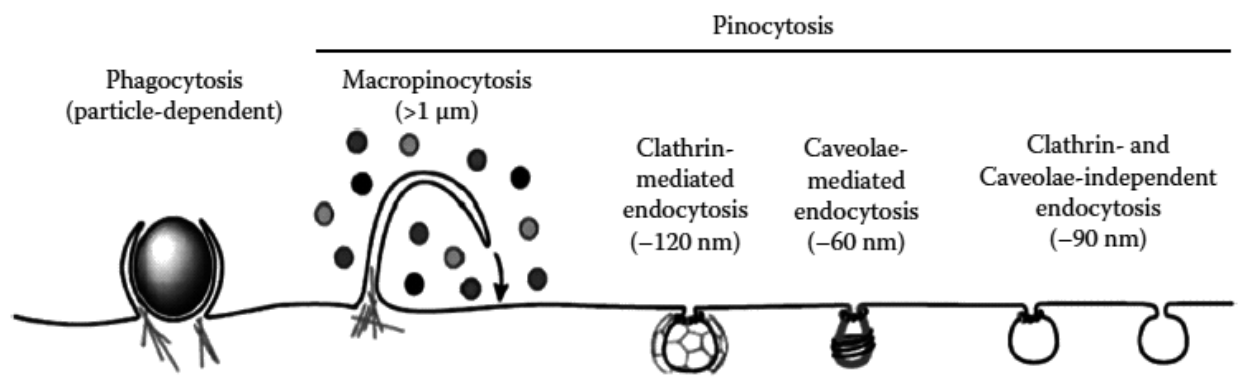

Figure 2. Various pathways of endocytosis

\section{Models for drug absorption}

Various in vitro, in situ, and in vivo tools and techniques are used to characterize the absorption of drug substance to determine the rate and extent of absorption.

Various models from low-throughput (in situ rat model) to high-throughput (in silico) models are used. Screening models for absorption such as human colon adenocarcinoma cell lines Caco-2 and HT-29 are widely used; recently, MDCK cell line is used as an alternative one.

Other in vitro methods are:

\section{Cell culture models}

In vitro cell culture models have been utilized to assess the permeability and metabolism of drugs, to elucidate molecular mechanism of drug transport to provide information on pathways of drug degradation, and to explore the influence of structure in the absorption of new chemical entities.

Several human colon carcinoma cells lines, such as the Caco-2, HT-29, SW116, LS174T, and SW480, are investigated for absorption. The cultured epithelial cells undergo enterocyte-like differentiation in culture and spontaneously differentiate into polarized columnar cells that are representative of the small intestine, with developed microvilli and polarized distribution of brush border enzymes. When grown on plastic membrane, epithelial cells result in a confluent monolayer and therefore serve as a model to study drug absorption. 


\section{Isolated mucosal cells}

Isolated mucosal cell suspensions have been used to study enzyme activity, drug transport, and cellular metabolism. The use of mucosal cells in drug absorption and transport studies is limited due to rapid autolysis.

\section{Brush border membrane vesicles}

Isolation of brush border membrane vesicles has been used extensively to study mucosal uptake process especially to investigate factors that influence mucosal uptake without interference of intracellular metabolism.

\section{Everted tissue approach}

a. Everted sac technique: To prepare everted sac, a small length of the intestine is excised, turned inside out, filled, and ligated at both ends. The sac is immersed in an oxygenated solution that contains a drug. The fluid inside the sac is assayed for the drug, and the rate of drug transfer across the membrane provides an estimate of drug permeability.

b. Intestinal rings: Prepared by excising a portion of the intestine, everting it over a glass road, and cutting it into rings approximately $30-50 \mathrm{mg}$. The rings are then incubated in an oxygenated culture media that contain a drug. At the end of the incubation, the tissues are extracted and the unchanged drug is measured. Intestinal ring preparation can be used to measure the rate of uptake and accumulation of a drug from the intestines.

\section{Isolated tissue technique}

In this technique, the epithelium is mounted as a flat sheet between two chambers. The solution on each side of the chamber is oxygenated and maintained at physiological temperature. The test drug and markers for volume fluctuation or tissue viability are placed in the chambers. The chambers can be stirred using a gas lift of $\mathrm{O} / \mathrm{Co} 2(95 \% / 5 \%)$ at a flow rate of $15-2 \mathrm{ml} / \mathrm{min}$. Samples can be obtained from the serosal and mucosal chambers to study diffusion and permeability.

In situ methods

1. Closed-loop studies

2. Perfused-loop studies

3. Perfused intestine-liver preparations [12, 22-25]

\section{Distribution}

Distribution provides information on the extent and time course of tissue accumulation and the elimination of drug and/or its metabolites.

The disposition of drug into the organs and tissues via circulation depends upon the nature of the drug. The more lipophilic the drug is, the better will be the distribution into the organs 
and tissues. Hydrophilic drugs are normally concentrated in cells and they are referred to as ion trapping.

When a drug is introduced into the body, the rate of distribution is dependent upon the following:

1. Tissues with the highest blood flow receive the drug: The rate at which a drug is distributed to various organs after a drug dose is administered depends largely on the proportion of cardiac output received by the organs.

2. Protein binding: Binding to proteins is inevitable in the case of drugs particularly lipoproteins, glycoproteins, and $\beta$-globulins. The extent of binding depends on the affinity of the drug molecule with the protein, and the maximum affinity could be $99 \%$ also. Unbound drug diffuses in the liquids surrounding the cells.

3. Lipid solubility: Lipid solubility is a major factor affecting the extent of drug distribution, particularly to the brain, where the blood-brain barrier restricts the penetration of polar and ionized molecules. Highly lipid-soluble drug can enter the tissues.

4. Molecular size: Molecular size is a factor affecting the distribution of extremely large molecules.

5. Distribution depends upon the ionization of drug, whereas unionized drugs can go anywhere into the body.

\section{Reasons for the variation in concentration of drug distribution are:}

1. Tissue differences in rates of uptake of drugs: Blood flow and capillary permeability

2. Differences in tissue/blood ratios at equilibrium: Dissolution of lipid-soluble drugs in adipose tissue, binding of drugs to intracellular sites, and plasma protein binding

3. Apparent volume of distribution $\left(\mathrm{V}_{\mathrm{d}}\right)$

\subsection{Volume of distribution}

The volume of distribution $\left(\mathrm{V}_{\mathrm{d}}\right)$, also known as apparent volume of distribution, is a pharmacological, theoretical volume that the total amount of administered drug would have to provide the same concentration as it is in blood plasma.

If the amount of drug $(X)$ and the resulting concentration $(C)$ are known, then the volume of distribution $\left(V_{d}\right)$ can be calculated using the simplified equations:

$X=V_{d} C$, where $X=$ amount of drug in body, $V_{d}=$ volume of distribution, and $C=$ concentration in the plasma.

Lipid-insoluble drugs are mainly confined to the plasma and interstitial fluid; most do not enter the brain following acute dosing. Lipid soluble drugs reach all compartments and may accumulate in fat. For drugs that accumulate outside the plasma compartment, $\mathrm{V}_{\mathrm{d}}$ may exceed the total body volume. 
Factors involved in drug distribution and diffusion across blood tissue barrier are:

1. Blood flow

2. Permeability across blood tissue barrier

3. Tissue solubility

4. $\mathrm{pH}$ partition

5. Protein binding within compartment

In our body, various structures are acting as reservoir for storage of drug substance. They are plasma proteins, erythrocytes, and cellular reservoir like muscles, fat tissue, bone, and transcellular compartments.

Multiple paths of drug distribution in the blood stream are shown in Figure 3.

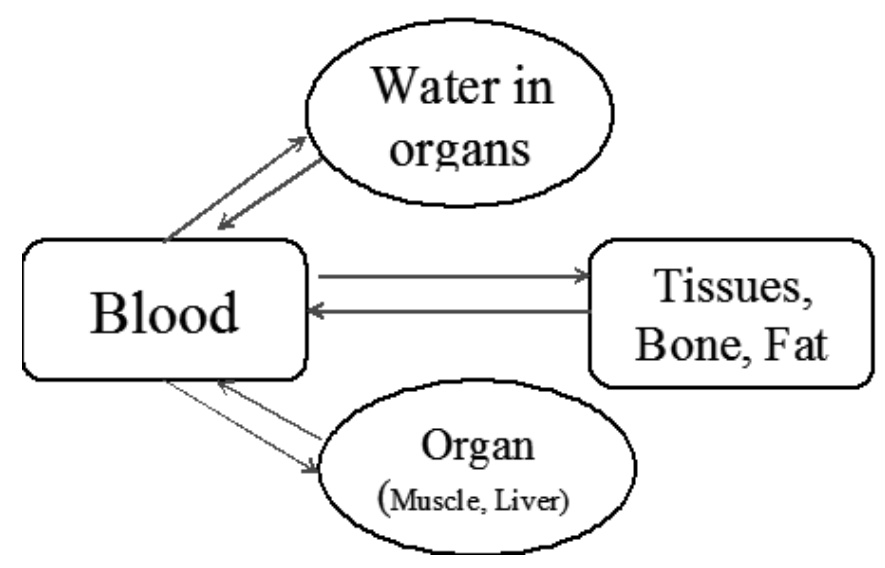

Figure 3. Multiple paths of drug distribution in the blood stream

\subsection{Compartment models in kinetics of drug distribution}

Compartment models are hypothetical structures used to describe the fate of a drug in a biological system after its administration into the body. Various compartment models in pharmacokinetic are:

One-compartment model: Following drug administration, the body is depicted as a kinetically homogeneous unit.

Two-compartment model: The two-compartment model resolves the body into a central compartment and a peripheral compartment.

Multicompartment model: In this model, the drug distributes into more than one compartment and the concentration-time profile shows more than one exponential [9, 15, 26-29]. 
Various body compartments and the drug distribution is shown in Table 1.

\begin{tabular}{ll}
\hline Body compartments (L/kg body weight) & Drug distribution in the body compartments \\
\hline $\begin{array}{l}\text { Total body water }=0.6 \\
\text { (extracellular and intracellular) }\end{array}$ & Small water-soluble drugs \\
\hline Extracellular water $=0.2$ & Larger water-soluble drugs \\
\hline Blood $=0.08$ & Plasma protein-bound large drugs \\
Plasma $=0.04$ & \\
\hline Fat $=0.2-0.35$ & Lipid-soluble drugs \\
\hline Bone $=0.07$ & Certain ions \\
\hline
\end{tabular}

Table 1. Body compartment and the drug distribution

\section{Biotransformation/Metabolism}

Biotransformation or drug metabolism is the enzyme-catalyzed conversion of drugs to their metabolites. Metabolism makes the drug less polar; lipid-soluble substance makes it more polar as well as water soluble, thus facilitating their excretion by the kidney. If a drug is already highly polar and water soluble, then it may not get metabolized and may get excreted as such. Liver is the chief organ for biotransformation of most drugs, but drug-metabolizing enzymes are found in many other tissues, including the gut, kidneys, brain, lungs, and skin. Lipophilic drug is converted to a hydrophilic one by extensive metabolism in the liver.

Drug metabolism is traditionally carried out by phase I and phase II processes.

Cytochrome P450 system has an important role and occupies a pivotal role in drug clearance in phase I.

Phase I: First step in biotransformation is the formation of product susceptible to phase II conjugative reaction. The phase I also involves unmasking a functional group like $\mathrm{OH}, \mathrm{NH}_{2}$ and $\mathrm{SH}$ and conversion to more polar products which may be mostly inactive, less active, and modified activity.

Phase II: Coupling of drug or its oxidized metabolite to endogenous conjugating agent derived from carbohydrate, protein, or sulfur sources; generally products are more water-soluble and more readily excreted in urine or bile. Phase II involves conjugation reactions with glucuronic acid, sulfuric acid, acetic acid, and amino acid.

Biotransformation occurs somewhere between absorption and excretion; some may occur in the gut (digestion, decomposition in gastric acidity).

Role of enzymes in the biotransformation are drug metabolism; conversion of prodrug to active forms; synthesis of steroidal hormones, cholesterol, and bile acids; and finally formation and excretion of bilirubin. 
Biotransformation is mediated by cellular enzymes in the sarcoplasmic reticulum, mitochondria, cytoplasm, lysosomes, and nucleus.

Drug-metabolizing enzymes are classified into:

1. Microsomal (inducible)

2. Nonmicrosomal (non-inducible)

\subsection{Microsomal enzymes (inducible)}

Microsomes are artificial spheres obtained from the endoplasmic reticulum by homogenization and fractionation, and they possess various drug-metabolizing enzymes.

1. Mixed-function oxidases (monooxygenases) cytochrome P-450, cytochrome P-450 reductase, and NADPH

Reactions catalyzed by monooxygenase are N-dealkylation, O-dealkylation, aromatic ring oxidation, side-chain oxidation, sulfoxide formation, N-oxidation, N-hydroxylation, deamination of primary and secondary amines, and desulfuration ( $\mathrm{S}$ replacement by $\mathrm{O}_{2}$ ).

2. Glucuronyl transferase for conjugation

The drugs containing phenols, alcohols, and carboxylic acids are metabolized by conjugation method. The conjugates are mostly inactive and excreted in the bile and urine by anion carrier mechanism and enter into enterohepatic cycling ( $\beta$-glucuronidase and sulfatase in the gut).

3. Some enzymes are involved in reduction and hydrolysis

The modification of enzyme activity such as enzyme induction and enzyme inhibition was observed.

Majority of the drugs however are metabolized by the nonmicrosomal enzymes resulting in their activation, inactivation, or modification. The reactions are:

1. Inactivation by conjugation: Synthetic process by which a drug or its metabolite is combined with an endogenous substance.

2. Inactivation by oxidation: Involves introduction of a hydroxyl group into the drug molecule.

3. Inactivation by reduction: Many halogenated compounds and nitrated compounds are reduced by microsomal enzymes.

4. Inactivation by hydrolysis: Carried out by enzyme esterase; this hydrolyses the esters.

Drug metabolism is affected by various factors. The diseases that are categorized as acute and chronic liver diseases (reduces metabolism), liver cancer, cardiac diseases limiting blood flow to the liver, pulmonary diseases reducing hydrolysis of procainamide, and hyperthyroidism where metabolism are affected. And also metabolism increases $t_{1 / 2}$ and hypothyroidism reduces metabolism $\mathrm{t}_{1 / 2}[14,30-36]$. 


\subsection{Metabolism methodologies}

\subsubsection{In vitro methods}

In vitro techniques are well suited for the study of biochemical toxicology, cytotoxicity, irreversible drug protein binding, drug metabolism, and enzyme regulation. Induction of drug-metabolizing enzymes can have a dramatic impact on the disposition, toxicology, and metabolic profile of the agent under study.

Primarily hepatic enzymes from animals and humans are used for drug metabolism studies. Other enzymes from the intestine and brain are also being used in the metabolism studies. In human, cytochrome P450 is used primarily, whereas its subfamilies such as CYP1A, CYP2C, CYP2D, CYP2E, CYP3A, and CYP4A are also being used.

Enzyme systems: Single or isolated enzyme systems are a powerful technique for the study of enzymatic process due to easy maintenance and manipulation in the substrate, enzyme, and cofactor concentrations. Interested enzyme from animal or human tissue can be isolated by extraction and purification and reconstituted to study the drug metabolisms. Single-enzyme system is useful in the study of enzyme kinetics, specificity, and mechanism. Other enzymes such as cytochromes CYP450, flavin-containing monooxygenases, glucuronyltransferases, sulfotransferases, epoxide hydrolases, glutathione S-transferases, and $\mathrm{N}$-acetyltransferases are also used in the drug metabolism studies.

Subcellular fractions: Microsomes as subcellular fraction is frequently utilized as in vitro model. These subcellular components, composed of endoplasmic reticulum, contain most of the oxidative drug-metabolizing enzymes, such as the cytochromes P450 and flavin monooxygenases, glucuronyltransferase, epoxide hydrolases, alcohol dehydrogenases, esterases, and methyltransferases, that can be separated by cell disruption and differential centrifugation.

Cellular systems: Cell culture system is utilized to study both drug metabolism and toxicology within a physiological environment due to manipulation of its enzyme concentrations and cofactors under appropriate conditions. These systems can be used to evaluate multiple aspects of drug metabolism, drug transport across cell membranes, enzyme induction, and cytotoxicity from such organs as the kidney, intestinal mucosa, and liver.

Liver slices: Organ slices were extensively used to study a variety of biochemical process because of the ability to produce uniform-cut organ slices by commercial tissue slicers and improved organ culture conditions. The slices have been isolated from many different species including human, and several organs such as the liver, brain, heart, and kidney are used.

Organ perfusion: Organ perfusion is used to measure the toxicological and pharmacokinetic events and parameters because of its close approximation to the tissues. This perfusion method offers several advantages over other in vitro methods such as preservation of organ architecture and ability to regulate perfused flow rate; two sampling sites are available for determination of substrate and metabolite concentrations.

But the limitation is that only one experiment can be performed per animal. 


\subsubsection{In vivo methods}

Radionuclides: Formation and excretion of metabolites can be easily monitored by attaching radiotracer tag on a drug candidate. Radiotracer tag is placed at chemically and metabolically stable site. Tritium $\left({ }^{3} \mathrm{H}\right)$ and carbon14 $\left({ }^{14} \mathrm{C}\right)$ are the most commonly used radionuclides used as tracer tag in drug metabolism studies [37].

\section{Clearance (Elimination)}

Drug clearance (CL) is defined as the volume of plasma in the vascular compartment cleared of drug (only free, i.e., not protein bound) per unit time by the processes of metabolism and excretion. Clearance is related to the concentrations of the drug present in blood after administration. Clearance of drug occurs by the perfusion of blood to the organs of extraction. Extraction is the ratio of the clearance process $(E)$ referring to the proportion of drug presented to the organ which is removed irreversibly (excreted) or altered to a different chemical form (metabolism) from the organ.

Hepatic clearance $\left(\mathrm{Cl}_{\mathrm{H}}\right)$ and renal excretion $\left(\mathrm{Cl}_{\mathrm{R}}\right)$ are generally involved in the extraction of the drug from the body. The overall value for systemic clearance $\left(\mathrm{CI}_{S}\right)$ can be calculated by

$$
\mathrm{Cl}_{\mathrm{S}}=\mathrm{Cl}_{\mathrm{H}}+\mathrm{Cl}_{\mathrm{R}}
$$

The amount of drug in the circulation is related to the volume of distribution, and therefore elimination rate constant $\left(k_{\mathrm{el}}\right)$ can be calculated by

$$
k_{e l}=C l / V_{d}
$$

Clearance for a drug is constant if the drug is eliminated by first-order kinetics.

Half-life: The time required to reduce the plasma concentration to one half its initial value is defined as the half-life $\left(t_{1 / 2}\right)$.

Zero-order reaction: The reaction proceeds at a constant rate and is independent of the concentration of drug present in the body.

First-order reaction: The reaction proceeds at a rate that is dependent on the concentration of drug present in the body.

\section{Excretory organs:}

Major routes: kidneys, liver, and lungs.

Minor routes: sweat, saliva, tears, and breast milk. 
Urine: It helps to quantitate the amount of drug excreted and is the most important excretory route for nonvolatile drugs and their metabolites (drug not bound to plasma proteins), proximal tubular active secretion, and passive tubular reabsorption.

Renal excretion: Small molecules with low molecular weight will appear in urine through glomerular filtration. Through tubular carrier systems (tubular secretion), a drug can be transported against the concentration gradient from the blood capillaries to the nephron lumen to be excreted in the urine.

Lipophilicity in drug clearance: Reduction in lipophilicity is observed when compared to the parent molecule during administration. For hydrophilic drugs ( $\log D_{7.4}$ below 0$)$, renal clearance is the predominant mechanism, whereas the drugs with $\log D_{7.4}$ values are above 0 , renal clearance decreases with lipophilicity. Metabolic clearance increases with increasing log $D$, and this becomes the major clearance route of lipophilic compounds. The lowest clearance (negligible) is observed below $\log D_{7.4}$ values of 0 by combined renal and metabolic processes $\left(\log D_{7.4}\right.$ Logarithm of the distribution coefficient $(D)$ at $\left.\mathrm{pH} 7.4\right)$.

Lipophilicity and reabsorption by the kidney: The degree of reabsorption (all along the nephron) depends on the physicochemical properties (degree of ionization and intrinsic lipophilicity) of the drug. After absorption, the equilibrium is reestablished in the kidney where the unbound drug in the urine and unbound drug in plasma are present on both sides of the membrane. The water-soluble drugs are absorbed easily, but lipophilic drugs will be reabsorbed by diffusion due to concentration gradient.

Effect of charge on renal clearance: Tubular $\mathrm{pH}$ is often more acidic ( $\mathrm{pH}$ 6.5) than plasma; hence, acidic drugs are reabsorbed more extensively than basic. Greater rates of excretion/clearance can occur for these charged moieties due to the tubular active transport proteins.

Renal clearance: The unbound drug will be cleared by filtration, and the protein-bound drug will be cleared slowly as it dissociates after a long time. Drugs with increasing plasma protein binding have increased lipophilicity, which decreases the renal clearance.

Renal clearance in drug design: Small molecules with relatively simple structures (molecular weights below 350) can successfully combine paracellular absorption and renal clearance.

Liver and biliary excretion: Liver is the organ where maximum metabolism takes place. The unabsorbed drugs and the metabolized drugs are excreted through fecal matter. Enzyme cytochrome is having a pivotal role in drug clearance by various oxidation reactions such as aromatic hydroxylation, aliphatic hydroxylation, $\mathrm{N}$-dealkylation, $\mathrm{O}$-dealkylation, $\mathrm{S}$-dealkylation, $\mathrm{N}$-oxidation, $\mathrm{S}$-oxidation, and alcohol oxidation. Hepatic and renal clearance process is shown in Figure 4.

Lungs: The lungs are an important route for the excretion of gaseous anesthetics, alcohol, iodine, and iodates.

Other excretion routes are sweat, saliva, and tears which are generally $\mathrm{pH}$ dependent that mediate drug excretion by passive diffusion of lipophilic drugs. 


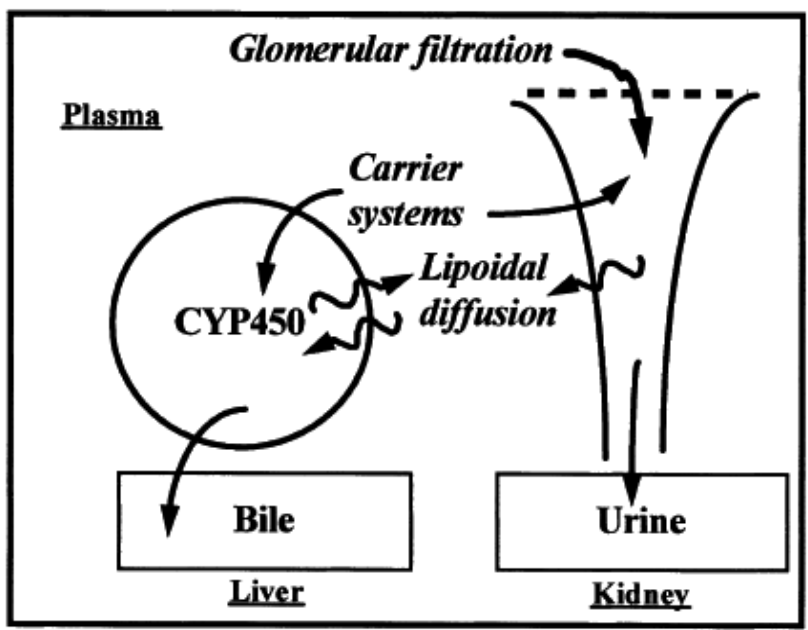

Figure 4. Hepatic and renal clearance process

Milk: Milk is more acidic than plasma; hence, basic drugs tend to accumulate due to ionic trapping, whereas concentration of acidic drugs is lesser than in the plasma. Nonelectrolytes (ethanol, urea) enter milk in a $\mathrm{pH}$-independent manner.

Hair and skin: Toxic metal may be excreted (murder, suicide) [8, 14, 29, 38-42].

\section{Conclusion}

Pharmacokinetics is the study of the time course of a drug within the body and incorporates the processes of absorption, distribution, metabolism, and excretion (ADME). The simplest pharmacokinetic concept is that based on concentration of drug in the biological matrix. Selective and sensitive bioanalytical method is required to quantify the concentration of the drug in the biological matrix. Most of the drugs are absorbed by passive diffusion process. The rate of drug diffusion by passive process depends upon the lipid solubility and the surface area available for absorption. The drug distribution is based on the plasma protein binding, molecular size, and lipid solubility. After distribution, the drug is metabolized into a metabolite as either a pharmacologically active or inactive one. The liver plays a vital role in the drug metabolism. Metabolized drugs are cleared mainly by the liver and kidney. The drug discovery and development process required a large amount of clinical data for rapid screening, selection, and development of new compounds. Various mathematical models are developed to assess the pharmacokinetic parameters. Preliminary pharmacokinetic study results are very much useful to characterize the absorption, disposition profile, and drug metabolism, which are very much essential and important in the discovery and development of new therapeutic agents in areas of currently unmet medical needs. 


\section{Author details}

S. Lakshmana Prabu ${ }^{1}$, T.N.K. Suriyaprakash ${ }^{2 *}, K_{\text {. Ruckmani }}{ }^{1}$ and R. Thirumurugan ${ }^{3}$

*Address all correspondence to: tnksuri@gmail.com

1 Dept. of Pharm. Technology, Bharathidasan Institute of Technology, Anna University, Tiruchirappalli, India

2 Dept of Pharmaceutics, Al Shifa College of Pharmacy, Kerala, India

3 School of Pharmacy, International Medical University, Malaysia

\section{References}

[1] Lakshmana Prabu S, Thirumurugan R, Suriyaprakash TNK. (2014). The role of the drug discovery, clinical, and regulatory affairs teams in turning a potent agent into a registered product. In: Elsevier Reference Module in Chemistry, Molecular Sciences and Chemical Engineering, Reedijk J (Ed.) Elsevier, Waltham, MA. 23-May-14 doi: 10.1016/ B978-0-12-409547-2.11400-3.

[2] Pharmaceutical Research and Manufacturers of America (PhRMA), Pharma FAX of the Week, May 2, 2003, RED Process: Long, Expensive, Risky Road.

[3] Regulatory control of active pharmaceutical ingredients. Version 1, Appendix 14.

[4] Pliska V, Testa B, Van de Waterbeemd H. (1996). Lipophilicity in Drug Action and Toxicology. VCH, Weinheim, Germany.

[5] Phytochemistry. (2012). In: Pharmacokinetics and Metabolism in Drug Design, Smith DA, Allerton C, Kalgutkar, Waterbeemed H and Walker DK, 1-17, Wiley-VCH Verlag \& Co, Weinheim, Germany.

[6] Hamilton HW, Steinbaugh BA, Stewart BH, Chan OH, Schmid HL, Schroeder R, et al., (1995). Evaluation of physicochemical parameters important to the oral bioavailability of peptide-like compounds: implications for the synthesis of renin inhibitors. Journal of Medicinal Chemistry, 38, 1446-1455.

[7] Gibaldi M. (1991). Preface. In: Biopharmaceutics and Clinical Pharmacokinetics. Lea, Febiger, 7, Malvern, PA.

[8] Panchagnula R, Thomas NS. (2000). Biopharmaceutics and pharmacokinetics in drug research. International Journal of Pharmaceutics, 201, 131-150.

[9] Lin JH, Lu AY. (1997). Role of pharmacokinetics and metabolism in drug discovery and development. Pharmacology Review, 49, 403-449. 
[10] Gunaratna C. (2000). Drug metabolism and pharmacokinetics in drug discovery: a primer for bioanalytical chemists, part I. Current Separation, 19, 17-23.

[11] Pharmacokinetic. (2012). In: Pharmacokinetics and Metabolism in Drug Design. Smith DA, van de Waterbeemd H, Walker DK, Mannhold R, Kubinyi H, Timmerman H, 19-40, Wiley-VCH Verlag GmbH, Weinheim, Germany.

[12] Lakshmana Prabu S, Suriya Prakash TNK. (2012). Extraction of drug from the biological matrix: a review. In: Applied Biological Engineering Principles and Practice, Naik GR, 479-506, InTech, Croatia.

[13] Absorption. (2012). In: Pharmacokinetics and Metabolism in Drug Design. Smith DA, van de Waterbeemd H, Walker DK, Mannhold R, Kubinyi H, Timmerman H, 41-59, Wiley-VCH Verlag GmbH, Weinheim, Germany.

[14] General pharmacology. (1993). In: Pharmacology and Pharmacotherapeutics. Satoskar RS, 1-50, Popular Prakashan Private Ltd, Mumbai.

[15] http://www.us.elsevierhealth.com/media/us/samplechapters/9781416066279/Chapter \%2002.pdf [Accessed on 10.10.2014]

[16] http://ocw.mit.edu/courses/health-sciences-and-technology/hst-151-principles-ofpharmacology-spring-2005/lecture-notes/ln34hms3275.pdf

[17] Birkett DJ. (2002). Pharmacokinetics Made Easy, $2^{\text {nd }}$ ed. McGraw-Hill, Roseville, Australia.

[18] Levine RR. (2000). Pharmacology: Drug Actions and Reactions, $6^{\text {th }}$ ed. Parthenon, New York.

[19] Clark B, Smith DA. (1986). In: An Introduction to Pharmacokinetics, $2^{\text {nd }}$ ed. Blackwell Scientific, Oxford.

[20] Jegadeesan D, Eswaramoorthy M. (2011). Nanomaterials for therapeutic drug delivery. In: Nanobiomaterials Hand Book. Balaji S, 12.1-12.22, CRC Press, Taylor \& Francis, UK.

[21] Absorption of drugs. (1995). In: Biopharmaceutics and Pharmacokinetics a Treatise. Brahmankar DM, Jaiswal SB, 5-75, Vallabh Prakashan, New Delhi.

[22] Hidalgo IJ. (1996). In: Models for Assessing Drug Absorption and Metabolism. Borchardt RT, Smith PL, Wilson G, 35-50. Plenum Press, New York.

[23] Artursson P, Palm K, Luthman K. (1996). Caco-2 monolayers in experimental and theoretical predictions of drug transport. Advanced Drug Delivery Review, 22, 67-84.

[24] Irvine JD, Takahashi L, Lockhart K, Cheong J, Tolan JW, Selick HE, et al., (1999). MDCK (Madin-Darby canine kidney) cells: a tool for membrane permeability screening. Journal of Pharmaceutical Sciences, 88, 28-33. 
[25] Habucky K. (2005). Methods to Assess Absorption in Drug Discovery, In: Pharmacokinetics Regulatory-Industrial-Academic Perspective. Welling PG and Tse FLS, 21-37, Vol. 67, Marcel Dekker, NY.

[26] Distribution. (2012). In: Pharmacokinetics and Metabolism in Drug Design. Smith DA, van de Waterbeemd H, Walker DK, Mannhold R, Kubinyi H, Timmerman H, 61-79, Wiley-VCH Verlag GmbH, Weinheim, Germany.

[27] Distribution of drugs. (1995). In: Biopharmaceutics and Pharmacokinetics a Treatise. Brahmankar DM, Jaiswal SB, 76-90, Vallabh Prakashan, New Delhi.

[28] Urso R, Blardi P, Giorgi G. (2002). A short introduction to pharmacokinetics. European Review for Medical and Pharmacological Sciences, 6, 33-44.

[29] Compartment modeling. (1995). In: Biopharmaceutics and Pharmacokinetics a Treatise. Brahmankar DM, Jaiswal SB, 230-272, Vallabh Prakashan, New Delhi.

[30] Biotransformation of drugs. (2001). In: Pharmacokinetics and Metabolism in Drug Design. Smith DA, van de Waterbeemd H, Walker DK, Mannhold R, Kubinyi H, Timmerman H, 75-98, Wiley-VCH Verlag GmbH, Weinheim.

[31] Ingelman-Sundberg M, Oscarson M, McLellan RA. (1999). Polymorphic human cytochrome P450 enzymes: an opportunity for individualized drug treatment. Trends Pharmacology Science, 20, 342-349.

[32] Bertilsson L. (1995). Geographical/interracial differences in polymorphic drug oxidation: current state of knowledge of cytochromes P450 (CYP) 2D6 and 2C19. Clinical Pharmacokinetic, 29, 192-209.

[33] Guengerich FP. (2003). Cytochromes P450, drugs, and diseases. Molecular Interventions, 3, 194-204.

[34] Handschin C, Meyer UA. (2003). Induction of drug metabolism: the role of nuclear factors. Pharmacology Review, 55, 649-673.

[35] Burchell B. (1999). Transformation reaction: glucuronidation. In: Handbook of Drug Metabolism, Woolf TF, 153-173, Marcel Dekker, New York.

[36] Benet LZ, Oie S, Schwartz JB. (1995). In: Goodman and Gillman's The Pharmacological Basis for Therapeutics, 1707-1792, McGraw-Hill, New York.

[37] Hayes RH, Pool WF, Sinz MW, Woolf TF. (2005). Recent developments in drug metabolism methodology. In: Pharmacokinetics Regulatory-Industrial-Academic Perspective. Welling PG, Tse FLS, 201-234, Vol. 67, Marcel Dekker, NY.

[38] Clearance. (2012). In: Pharmacokinetics and Metabolism in Drug Design. Smith DA, van de Waterbeemd H, Walker DK, Mannhold R, Kubinyi H, Timmerman H, 81-102, Wiley-VCH Verlag GmbH, Weinheim, Germany. 
[39] Renal clearance.. (2012). In: Pharmacokinetics and Metabolism in Drug Design. Smith DA, van de Waterbeemd H, Walker DK, Mannhold R, Kubinyi H, Timmerman H, 103-110, Wiley-VCH Verlag GmbH, Weinheim, Germany.

[40] Excretion of drugs. (1995). In: Biopharmaceutics and Pharmacokinetics a Treatise. Brahmankar DM, Jaiswal SB, 178-203, Vallabh Prakashan, New Delhi.

[41] Pharmacokinetic basic considerations. (1995). In: Biopharmaceutics and Pharmacokinetics a Treatise. Brahmankar DM, Jaiswal SB, 212-229, Vallabh Prakashan, New Delhi.

[42] http://pharmaquest.weebly.com/uploads/9/9/4/2/9942916/pkinetic_parameter.pdf [Accessed on 10.10.2014] 

Chapter 6

\title{
Clinical Pharmacokinetic Applications of Recirculatory Models
}

\author{
José M. Lanao and Clara Isabel Colino \\ Additional information is available at the end of the chapter \\ http://dx.doi.org/10.5772/61156
}

\section{Introduction}

In clinical practice, conventional pharmacokinetic analysis usually relies on the use of classic pharmacokinetic procedures, such as compartmental models. The main advantage of these models in clinical practice is their excellent capacity to predict the concentrations of drugs from different doses and administration conditions. Also the relative ease with which they can be optimized using polyexponential analysis. Moreover, this type of model affords information about the fundamental pharmacokinetic parameters of disposition, such as elimination clearance or apparent distribution volumes, which can be correlated with the demographic (age, weight, etc.) or clinical variables (renal function, hepatic function, etc.) that may be altered in disease states. The principal drawback of compartmental models is that they fail to reflect physiological reality, which hinders the interpretation of the biological alterations induced by disease states and the influence of these in pharmacokinetics. Among the physiological variables affected by disease states are regional blood flow, renal and liver extraction, extracellular water volume and membrane permeability, etc., all of which contribute to changes in drug plasma and tissue levels, and in the pharmacological response.

Physiological pharmacokinetics contributes to the interpretation of the influence of physiological and biochemical changes in the pharmacokinetic behavior. For more than a hundred years, the indicator-dilution method was proposed to measure blood flow. Different authors, however, have suggested the possibility of using those methods to measure physiological variables [1]. The indicator-dilution theory, combined with the concept of drug recirculation, permitted the development of the recirculatory models [2].

Recirculatory models are a type of so-called physiologically based pharmacokinetic models (PBPK). Analysis of drug disposition based on blood recirculation incorporates important physiological factors affecting the pharmacokinetics, such as cardiac output. The fundamental 
assumption is that there is a circulatory transport function that may be considered as a probability density function of transit times after a first passage through the system. In the recirculatory models, drug disposition is analyzed as the convolution of its circulatory transport function [2]. Clinical application of the recirculatory models in the field of pharmacokinetics was proposed by Weiss [3]. Currently, clinical application of the these models is quite varied, especially in the field of anesthesiology [4-6] and contributes to the analysis and interpretation of clinical pharmacokinetic data.

\section{Theoretical considerations}

Recirculatory models are a type of physiological modelling alternative to classical compartmental modelling and they use physiological concepts such as circulatory transport function, cardiac output, and systemic extraction by elimination organs such as the liver and the kidney. These models arise from the tracer indicator-dilution theory combined with the concept of drug recirculation. This method was developed by Hearing in 1824 to measure the blood flow. In the early $20^{\text {th }}$ century, and after the work of Stewart in 1897 and later Hamilton, it was used to estimate clinical hemodynamic blood flow $[7,8]$. Variants of these methods have been used to study the physiology of the circulation and their pathological changes, including cardiac output. A marker such as indocyanine green (ICG) (Stewart-Hamilton method) or thermodilution can be used, by measuring temperature instead of concentration after injecting a saline solution at low temperature [9]. These models assume the existence of circulatory transport functions that may be modelled by empirical functions.

\section{Concept of circulatory transport function}

The circulatory transport function represents the density function of the transit times of the molecules in the first passage through the system or subsystem. Its profile depends on the injection and sampling sites in the vascular system.

Assuming a stochastic analysis of drug transport through a single-blood perfused organ without recirculation, when one unit of drug is injected as a single pulse a transport function $f(t)$ may be defined $[10,11]$. According to the tracer kinetic theory, the circulatory transport function represents the transit time density function of the molecules travelling from the beginning to the end of the organ or system. The central tendency of the transport function is given by the mean transit time and dispersion can be characterized by the variance in transit times $[12,13]$.

The zero, first, and second moments of the circulatory transport function correspond to the area under the curve $(A U C)$, the mean transit time (MTT), and the variance of transit times $(V T T)$, respectively, and may be calculated as: 


$$
A U C=\lim _{s \rightarrow 0} f(s)
$$

and

$$
M T T=\lim _{s \rightarrow 0}-d / d s \ln f(s)
$$

and

$$
V T T=\lim _{s \rightarrow 0}-d^{2} / d s^{2} \ln f(s)
$$

where $f(s)$ represents the function transport expressed in the Laplace domain.

The relative dispersion of the tracer $\left(R D^{2}\right)$ may be calculated as a normalized variance of the transit times according to the following expression [14]:

$$
R D^{2}=\frac{V T T}{[M T T]^{2}}
$$

The relative dispersion $\left(R D^{2}\right)$ may be used for the interpretation of the intravascular mixing of markers [14, 15].

According to the linear systems theory, the concentration-time curve at the output of the organ $C(t)$ is the convolution of the input function $f_{I}(t)$ and the transport function $f(t)$ according to the following expression [11]:

$$
C(t)=\int f_{I}(\tau) f(t-\tau) d \tau
$$

In the Laplace domain this equation is expressed as:

$$
C(s)=f_{I}(s) \cdot f(s)
$$

Depending on the kind of input used, $f_{I}(s)$ may take the following expressions:

$$
f_{I}(s)=\text { Dose }
$$

when the drug is administered as an instantaneous i.v. bolus injection, or 


$$
f_{I}(s)=\frac{\left(\text { Dose } / T_{\mathrm{inf}}\right)}{s} \cdot\left(1-e^{-T_{\mathrm{inf}} \cdot s}\right)
$$

when the drug is administered by i.v. infusion with zero-order input, where $T_{\text {inf }}$ is the infusion time.

Different approaches to the circulatory transport function, either model-dependent or independent, have been made $[2,12,16]$. Some empirical approaches to the circulatory transport function of tracers in each subsystem, such as inverse Gaussian, log-normal, Gaussian, or Erlang functions have been proposed, as shown in Table 1 [17-20].

\begin{tabular}{ll}
\hline Inverse Gaussian & $f(t)=\sqrt{\frac{M T T}{2 \pi R D^{2} t^{3}}} \exp \left\{-\left[\frac{(t-M T T)_{i^{2}}}{2 R D^{2} M T T t}\right]\right)$ \\
\hline Gaussian & $f(t)=\frac{1}{\sigma \sqrt{2 \pi}} e^{-\frac{1}{2}\left[\frac{t-t_{0}}{\sigma}\right]^{2}}$ \\
\hline Erlang & $f(t)=\frac{k^{n} t^{n-1}}{\Gamma(n)} e^{-k . t}$ \\
\hline Log-Normal & $f(t)=\frac{1}{\sigma \sqrt{2 \pi \sigma \cdot t}} e^{\left(-\frac{1}{2 \sigma^{2}}\left[\ln \frac{t}{\mu}+\frac{\sigma^{2}}{2}\right]^{2}\right)}$ \\
\hline
\end{tabular}

MTT: mean transit time, $\mathrm{RD}^{2}$ : relative dispersion, $\mu$ : mean, $\sigma$ : variance, $\mathrm{k}$ : first order rate constant.

Table 1. Empirical equations used for the characterization of the circulatory transport function of tracers in different subsystems.

In addition, mathematical dispersion-convection models using partial differential equations have been proposed. Such models have been used for the modelling and simulation of the circulatory transport function of tracers after a single-pass, depending on the administration/ sampling sites [21]. The parameters of the circulatory transport function may be estimated using nonlinear regression procedures.

The circulatory transport function combined with a recirculation model allows predictions to be made about the initial distribution of a drug after its first passage through the systemic circulation and also permits the plasma levels curve after recirculation in the body to be generated.

\section{Recirculatory models}

From a physiological standpoint, the circulatory system consists of two circuits through which blood flows: pulmonary and systemic. The pulmonary circuit moves the blood to the lungs to 
be oxygenated and then returns it to the heart. The systemic circuit moves the blood between the heart and the rest of the organs and tissues of the body.

The body can be considered as a system of parallel compartments perfused with arterial blood, which is recirculated via the venous blood. Each compartment represents organs and tissues such as kidney, liver, muscle, etc., or groups of organs connected in parallel or in series. Blood flow between the compartments goes from the arterial line to the venous line and the recirculation of blood from the venous blood line passes through the heart-lung system as shown in Figure 1. Assuming the body to be a closed-loop system, the drug is injected into the venous system, circulating through the heart and pulmonary circulation, returning to the heart and the arterial system, and finally returning to the venous system.

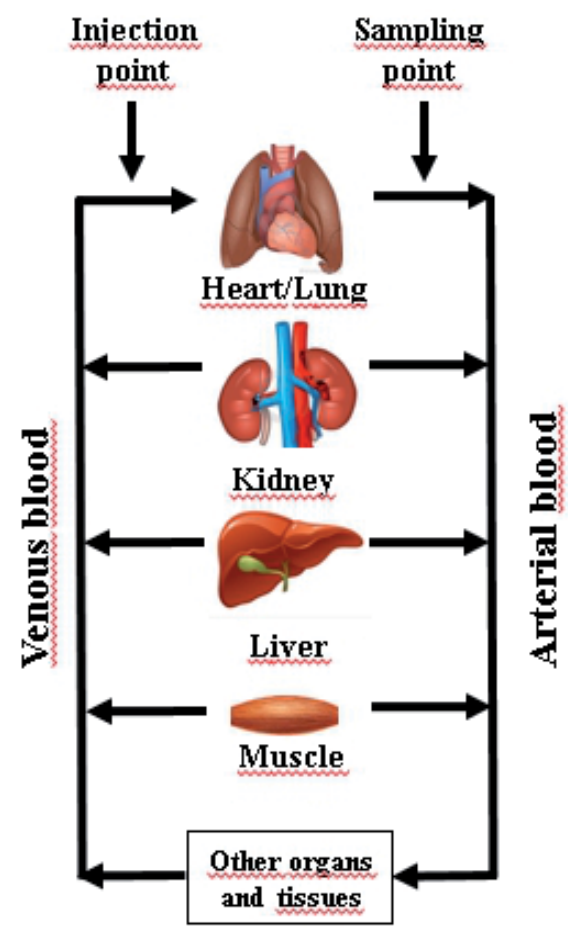

Figure 1. Scheme of the circulatory system and the recirculation of blood. The tracer is administered by bolus intravenous injection and the sampling is made at the arterial line.

Depending on the injection and sampling points, the space covered by the drug in its first passage through the systemic circulation can be variable, conditioning the profile of the circulation transport function. Considering the circulatory system as a linear one, and from a mathematical point of view, the recirculatory process is considered to be the convolution of the circulatory transport function in the different subsystems [2].

Different kinds of pharmacokinetic recirculatory models with experimental and clinical applications have been proposed. For the development and validation of recirculatory models 
many types of markers have been used. Indocyanine green (ICG) is frequently used as a marker of the vascular space; inulin is a marker of extracellular fluid, and antipyrine is used as a marker of the total body water [14].

From experiments using ICG in sheep, a recirculatory model based on the estimation of a body transport function was proposed. The tracer was injected into the right atrium of the heart and the dilution curve of ICG was measured using a catheter in the aorta. The model considers the body transport function to be composed of two different subfunctions associated with two parallel functional compartments of the body: namely, a fast compartment with a low transit time value and a slow compartment with a higher transit time [19].

From a physiological interpretation, a minimal recirculatory model including pulmonary and systemic circulation, as shown in Figure 2, has been proposed [15, 22, 23].

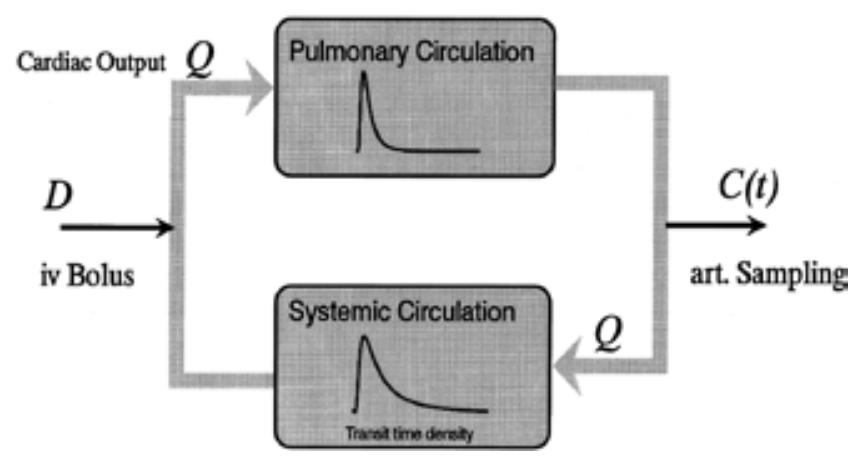

Figure 2. Minimal recirculatory model based on pulmonary and systemic circulation. Reproduced with permission from Elsevier [22].

In this model, the basic equation for the arterial concentration-time curve for infinite cycles in a closed loop system for a vascular space marker such as ICG after a standard input may be expressed in the Laplace domain by the following equation:

$$
C_{p}(s)=\left(f_{I}(s) / C O_{P}\right) \frac{f_{p}(s)}{1-(1-E) \cdot f_{s}(s) \cdot f_{p}(s)}
$$

$f_{p}(s)$ and $f_{s}(s)$ represent the Laplace transform of the pulmonary and systemic circulation transport functions, respectively; $E$ is the systemic extraction by elimination of the drug; $\mathrm{CO}_{p}$ is the plasma cardiac output; and $f_{I}(s)$ represents the Laplace transform of the input function of the drug in the body. The plasma concentration-time curve, $C_{p}(t)$, is finally obtained as the inverse Laplace transformation:

$$
C_{p}(t)=L^{-1}\left(C_{p}(s)\right)
$$


A more complex recirculatory model was developed to characterize the disposition of drugs having a wide tissue distribution with potential clinical applications. In addition to circulatory transport functions in the different subsystems, the model considers the processes of tissue distribution.

The basic form of this model includes the pulmonary and systemic circulations. Pulmonary circulation is characterized by an empirical transit time density function $f_{p}(s)$ and systemic circulation $f_{s}(s)$ is based on an axially distributed capillary-tissue exchange model with vascular and extravascular distribution volumes. Distribution parameters such as the tissue distribution volume $\left(\mathrm{V}_{\mathrm{T}}\right)$, the permeability-surface product $(\mathrm{PS})$, and diffusional transport into the tissue space using an equilibration time parameter (ds) may be characterized with this model. Flow-limited distribution kinetics of drugs such as antipyrine or diffusion-limited distribution kinetics of substances such as inulin may be modelled [24]. In the advanced version of the model, the systemic organs are grouped into two subsystems, non-fat tissues and fat tissues [23].

Recirculatory models incorporating dispersion concepts or models based on the fractal geometry of the vascular tree and the hydrodynamics of the blood flow have also been proposed. The spatiotemporal kinetics of tracers is described using partial differential equations incorporating dispersion and convection processes [21, 25, 26].

As an alternative, a pharmacokinetic recirculatory model based on the analysis of arterial and mixed venous data was proposed and applied successfully to characterize the pharmacokinetics of ICG, antipyrine and lidocaine. ICG andantipyrine have been used as markers of the vascular space and total body water, respectively. This model is based on catenary compartmental models linked in series [27-30].

As an alternative to the classical two-compartment mammillary model, a two-compartment recirculatory model has been proposed to characterize drug disposition [31]. In this model, the central compartment is defined by the lungs and the peripheral compartment by the rest of the body, without the lungs. The mass balance between compartments depends on the distribution volumes of both compartments, cardiac output, and the arterial $\left(C_{A}\right)$ and venous plasma concentrations $\left(C_{V}\right)[31]$.

This model has been used to fit the data set of arterial thiopental concentrations in sheep. They are considered to be the sum of two components: the first-pass arterial concentrations and the recirculated concentrations. A dynamic component can easily be added to the model by introducing a target organ for which there is a defined target organ concentrationeffect relationship. This design has been applied also for propofol [32]. Despite its simplicity, this model highlights the fact that slowing the bolus injection rate of a fixed dose can lead to significantly lower peak arterial concentrations. Also an inverse relationship was found between these concentrations and cardiac output [31]. More complex recirculatory models to characterize the disposition of drugs in specific tissues such as the brain have been proposed [33]. 
An important aspect of recirculatory models is that they are able to fit the data from arterial and venous blood samples obtained simultaneously. It is common in pharmacokinetic studies of anesthetics to include early arterial samples but for convenience later samples are taken from a vein. Although these venous drug concentrations are assumed to differ non-significantly from simultaneously sampled arterial concentrations, this is not always true, as found in a phase I study for remimazolam. [34]. In this study, the data on each subject included early arterial, late venous points, and samples from both at some middle points. Two population pharmacokinetic models were applied to the plasma levels: a conventional mammillary model with 3 or 4 compartments and a recirculation model. The compartmental models that assume that the drug is distributed instantaneously throughout the central compartment are only valid with compounds whose distribution and clearance kinetics are relatively slow compared with the blood flow. This was not the case of remimazolam, which showed significantly higher venous concentrations than arterial ones (ratio $>1,44$ ) [34]. Only a recirculatory pharmacokinetic model including both venous and arterial compartments, a cardiac blood/pulmonary compartment for the dose and clearance from the peripheral compartment was able to fit the data. Three- and four-compartment mammillary models gave much poorer fits [34].

A more complex, six-compartment hybrid physiological model was used to define propofol kinetics and dynamics [35].The model includes a description of initial bolus kinetics, lung kinetics and cardiac output, along with the effects of propofol-induced changes in cerebral blood flow and a combined description of systemic kinetics as two tissue pools. It was able to simulate the complex effects of circulatory changes on the pharmacokinetics and pharmacodynamics of propofol in sheep as well as in humans [32,33]. With this model it was shown that increased cardiac output resulted in decreased duration of anesthesia, whereas increased cerebral blood flow increased the depth (but not the duration) of anesthesia [36]. This was attributed to the influence of cardiac output on tissue distribution. On the basis of this evidence, it has been speculated that a reduction in cardiac output caused by co-administration of drugs such as midazolam and fentanyl to anxious patients, might be part of the mechanism by which these drugs reduce the required induction dose of propofol [37]. When applied to define propofol kinetics in a standard patient, the model was consistent with the clinical data and was able to predict other data not used in its development [33].

Recently, three pharmacokinetic compartmental models and one recirculatory model (Upton model) have been compared to simulate the time course of propofol plasma concentrations. Published Cp data from studies of both manual (bolus and short infusion) and computercontrolled (TCI) propofol dosing schemes were used. The performance indices of the models during the first 5 min after a bolus injection revealed a clear overprediction for all three compartmental models after $1 \mathrm{~min}$. The Upton model only performed reasonably well after 120 s. For short infusions, the recirculatory model did not provide accurate prediction of $\mathrm{Cp}$ before $1 \mathrm{~min}$ and after the termination of drug administration. This was attributed to the fact that the lag time was modeled from centrally administered propofol while in the datasets used the drug was administered peripherally. However, the authors concluded that more studies were required to assess the validity of the Upton model predictions for the first minute [138]. Although the Upton recirculatory model did not show any clear advantage in the fitting of the 
datasets employed, the authors pointed out its inherent interest since it should be able to predict the effect of common hemodynamic disturbances such as congestive heart failure, severe blood loss, dehydration, and other high and low cardiac output states on propofol pharmacokinetics, as previously reported for animal studies. However, this remains to be studied.

Krejcie and Avram [29] developed a recirculatory pharmacokinetic model using antipyrine as a surrogate for lipophilic drugs such as thiopental to study factors affecting the initial disposition of drugs with a rapid onset of effect. This model was used to study antipyrine disposition in dogs with altered cardiac output and blood flow distribution $[4,9,39,40]$. They found that not only cardiac output but also its peripheral distribution affects the early history of antipyrine concentrations after rapid intravenous administration [41].

This model was also applied in a study that simulated the usefulness of a recirculatory model compared with a three-compartment mammillary pharmacokinetic model, to design an adequate target-controlled drug infusion. They found that the fitting of the data was indistinguishable for both types of models. However, the mammillary model overestimated not only $\mathrm{Vc}$ but also $\mathrm{V}_{\mathrm{F}}$ and $\mathrm{CL}_{\mathrm{F}}$ (volumes and clearance for rapid-equilibrating tissues), which could lead to concentrations higher than the target concentrations. The use of a reasonable estimate of $V_{c}$ as the sum of $V_{c}$ and $V_{N D}$ (non-distributive distribution volume) from the recirculatory model was recommended. Accordingly, it was proposed that better estimates of Vc can be obtained from studies in which the drug of interest is administered in a short-term intravenous infusion in order to avoid many of the mistaken assumptions made when fitting data obtained after rapid intravenous drug administration [42].

The most realistic application of the recirculatory models involves frequent data collection after drug injection, including first-pass and recirculation peaks. This information allows the physiological changes induced by disease states that may affect the pharmacokinetic behavior of drugs and their influence on the pharmacological response to be interpreted.

Recirculatory models have some pitfalls for their practical clinical application such as:

1. Their greater numerical and computational complexity in comparison with classic models, since they often have to work with mathematical functions in the Laplace domain.

2. The complexity involved in performing experiments with plasma indicator dilution curves in humans, especially in clinical practice.

3. The difficulty inherent to estimating cardiac output or regional blood flows in patients.

4. The need to have a sufficiently large number of experimental data, which is also a limitation in clinical practice.

5. Greater computational difficulties, because conventional pharmacokinetic software is not appropriate for this type of model and it is necessary to use specific software based on the numeric inversion of the Laplace transform such as Scientist [43], FILT [44], or MAPLE [45] among others. 


\section{Recirculatory models and disease states}

Recirculatory models are a relatively unexplored alternative to classic pharmacokinetic analysis. Although there have been instances of their use in clinical practice, especially in the field of anesthesiology [4-6], their application in the analysis and interpretation of the physiological changes induced by disease states and their influence on pharmacokinetics is infrequent. The main advantage of recirculatory models as compared with classic models such as compartmental ones lies in their greater physiological basis in the sense that they handle parameters, such as cardiac output, that are directly related to the physiology of the body and the changes that may occur to it in disease states or clinical situations deriving from the diagnosis or treatment of the patient.

Many pathophysiological situations or disease states affect one or several parameters included in the recirculatory model equations such as cardiac output $\left(C O_{p}\right)$, regional blood flows $\left(Q_{T}\right)$, tissue distribution volumes $\left(V_{T}\right)$, tissue $\left(\mathrm{E}_{\mathrm{T}}\right)$ and systemic $\left(E_{S}\right)$ extraction. As a consequence, recirculatory model equations are very useful for simulating the influence of these variables in the plasma or serum levels of drugs.

Within the context of distribution and elimination kinetics, distribution $\left(C l_{D}\right)$ and systemic elimination $\left(\mathrm{Cl}_{\mathrm{s}}\right)$ clearances may be considered on the basis of the following equations:

$$
\begin{aligned}
& C l_{D}=C O_{p} \cdot E_{T} \\
& C l_{s}=C O_{p} \cdot E_{S}
\end{aligned}
$$

\subsection{Drug distribution}

Tissue distribution is affected by tissue extraction, which reflects the overall contribution of the different organs and tissues to the distribution of the drug. The tissue extraction coefficient, $E_{T}$, depends on factors such as regional blood flow, membrane permeability, and tissue binding. Overall tissue extraction may be related to extraction by a specific tissue $\left(E_{S T}\right)$, according to the following expressions:

$$
E_{T}=E_{S T} \cdot \frac{Q_{T}}{C O_{p}}
$$

and

$$
E_{S T}=\frac{E_{T} \cdot C O_{p}}{Q_{T}}=\frac{C L_{D}}{Q_{T}}
$$


where $Q_{T}$ represents the plasma perfusion to a specific tissue.

According to equations 13 and 14, changes in extraction by a specific tissue $\left(E_{S T}\right)$ due to alterations in regional perfusion, tissue permeability, or tissue binding induced by disease states will be reflected in the overall tissue extraction $\left(E_{T}\right)$ and, consequently, in the distribution clearance $\left(C L_{D}\right)$ of drugs.

In drugs with flow-limited distribution, the distribution clearance to specific tissues is equal to the regional blood flow. In this situation, the overall tissue extraction of equation 13 reflects the fraction of the plasma cardiac output that contributes to the tissue distribution of the drug.

Tissue blood flow is dependent on cardiac output, which can be modified in different disease states or in response to certain drugs. Cardiac output is moderately reduced with age and in the presence of cardiovascular diseases such as congestive heart failure, hypertension, and others. Some drugs may increase or reduce the cardiac output. For example, all anesthetics decrease cardiac output and distribution clearance [46].

The effect of reduced distribution clearance is to reduce the initial plasma concentrations after drug administration. This is particularly important in drugs with rapid onset such as anesthetics [47]. Recirculatory models may help to evaluate the initial distribution and pharmacodynamic response of this type of drugs.

Anesthetists frequently rely on drugs administered by intravenous bolus, whose effects start in 1-2 min. Thus, the first few minutes are particularly relevant in monitoring drug and side effects. It seems clear that the so-called front-end kinetics plays a large part in determining the rate and extent of drug distribution to the site of drug effect and that it is the early distribution kinetics that should be characterized most accurately [37].

Standard compartment mammillary models assume that a drug is mixed instantaneous and completely in the central compartment. This implies that for bolus administration the predicted plasma concentrations at time zero are the highest, whereas in reality they are zero. These models ignore the complexity of the early distribution processes, which depend on cardiac output and its distribution, tissue perfusion and affinity for a drug, and the relative concentrations of the drug in blood and tissues. Thus, they overestimate the actual initial distribution volume, which, along with the dose, determine early drug concentrations and the intensity of the drug effect. Accordingly, despite their widespread use to describe drug disposition they have some limitations when applied to the rapid administration of anesthetic drugs, particularly those with a rapid onset of effect [37].

Recirculatory pharmacokinetic models retain the relative simplicity (compared with physiological models) of mammillary models but incorporate descriptions of key physiologic processes that have been recognized as important determinants of IV anesthetic disposition [48]. The inclusion of pulmonary drug uptake, cardiac output, and its regional disposition enables recirculatory models to identify the pharmacokinetic basis of interindividual differences in response to IV anesthetics attributable to age, disease, or druginduced physiological disturbances. They can also be used to design target-controlled drug infusions (which do not result in early drug concentrations in excess of the target be- 
cause of overestimation of the initial distribution volume) and to estimate pharmacodynamic parameters with greater precision [47].

\subsection{Drug elimination}

As mentioned earlier, systemic elimination is influenced by systemic extraction, which reflects the overall contribution of the different elimination routes to the extraction of the drug. Systemic extraction is related to hepatic $\left(E_{H}\right)$ and/or renal extraction $\left(E_{R}\right)$ according to the following expressions:

$$
E_{S}=E_{H} \cdot \frac{Q_{H}}{C O_{p}}
$$

and

$$
E_{S}=E_{R} \cdot \frac{Q_{R}}{C O_{p}}
$$

Thus:

$$
\begin{aligned}
& E_{H}=\frac{E_{S} \cdot C O_{p}}{Q_{H}}=\frac{C l_{S}}{Q_{H}} \\
& E_{R}=\frac{E_{S} \cdot C O_{p}}{Q_{R}}=\frac{C l_{S}}{Q_{R}}
\end{aligned}
$$

where $Q_{H}$ and $Q_{R}$ represent the hepatic and renal plasma flows, respectively. According to equations 15 to 18 , changes in hepatic or renal extraction induced by disease states will be reflected in the systemic extraction and systemic clearance of the drug.

The hepatic extraction of a drug may depend on changes in hepatic blood flow, protein binding, or intrinsic clearance. All these factors may be altered in different disease states. In this sense, hepatic blood flow may be altered in patients with congestive heart failure or in patients with hepatic disease [49-53]. Protein binding and intrinsic clearance may also be affected in patients with liver disease [52, 54, 55].

The renal extraction of a drug may depend on changes in renal blood flow, glomerular filtration, protein binding or intrinsic clearance by tubular excretion, etc. These factors may also be affected in several disease states. Renal blood flow and the glomerular filtration rate are altered in patients with congestive heart failure [53] or hemorrhagic shock. Protein binding may be affected in different clinical situations, such as hepatic or renal failure [56]. Intrinsic clearance by tubular secretion is altered in different renal pathologies. 


\subsection{Clinical applications}

Recirculatory models usually use the plasma cardiac output of the patient, the parameters of the circulatory transport functions, and the systemic extraction coefficient as fundamental parameters as an alternative to classical elimination and distribution parameters. This offers a potential advantage over classic compartmental models since the model estimates the components of the elimination and distribution clearances separately; that is, cardiac output and systemic extraction. In clinical practice, the most suitable way of working with this model would be to estimate the plasma cardiac output of the patient by a thermodilution technique [57] and then introduce the estimated values into the function of the model in order to optimize the other pharmacokinetic parameters later by nonlinear regression. However, in most studies addressing clinical pharmacokinetics the cardiac output of the patient is not measured, such that a reference value must be assumed or optimized by nonlinear regression as a parameter of the model.

The modifications in systemic and distribution clearances induced by changes in plasma cardiac output or in the systemic and tissue extraction coefficients may affect plasma levels and the pharmacological response in different ways during the initial distribution of the drug or after a recirculation process.

Modifications in cardiac output may be responsible for changes in the elimination of drugs in different disease states, these not being accompanied by changes in the functionality of organs of elimination, such as the kidney or the liver. Thus, in situations such as congestive heart failure, hemorrhagic shock, or hypermetabolic states, alterations in the cardiac output or regional blood flow of patients that lead to a decrease or an increase in the drug elimination clearance may occur, with no modifications to the intrinsic elimination capacity of the liver or kidney. Additionally, changes in cardiac output may also account for variations in the distribution clearance of drugs when tissue blood flows are modified, as previously reported [58], where a correlation between the intercompartmental clearance of alfentanil and the measured cardiac output of the patient was found.

One of the advantages of recirculatory models is that they allow simultaneous evaluation of the changes occurring in the distribution and elimination of drugs as a result of changes in cardiac output.

Among other factors, the systemic extraction coefficient is related to the functional status of the organs of elimination, that is, the liver or kidney. The changes in the functionality of eliminating organs as a consequence of a given pathology will be reflected in the systemic extraction coefficient. Thus, modifications in the intrinsic metabolic clearance of a liver marker with low hepatic extraction, such as antipyrine, in hepatic diseases $[59,60]$ will be reflected in a reduction in the systemic extraction coefficient of the model, which in turn explains the reduction in metabolic clearance. Additionally, the reduction in renal functionality in patients with nephropathies will in turn be reflected in the systemic extraction coefficients of drugs that are eliminated through the kidney, such as the aminoglycoside antibiotics [60]. Moreover, changes in the regional blood flow of the liver or kidney and in drug protein binding induced by disease states may be reflected in the systemic extraction coefficient. Finally, changes in the 
tissue extraction coefficient of a drug in different types of patients reflect changes in the physiological factors that control tissue distribution, such as regional blood flow, membrane and cell permeability, or tissue binding, as a result of disease states.

Table 2 shows some disease states that can modify the cardiac output of the patient or in the tissue and systemic extraction or in the distribution volumes of the drug. Thus, in patients with chronic renal failure a reduction occurs in the systemic clearance of drugs that are wholly or partially eliminated through the kidney, such as the aminoglycoside antibiotics. This decrease in clearance is brought about by a decrease in renal functionality, reflected in a decrease in the systemic extraction coefficient. However, there are other disease states in which a reduction or an increase occurs in the systemic clearance of drugs due to a reduction or an increase in plasma cardiac output, as happens in patients with congestive heart failure or in patients with a hypermetabolic condition, respectively.

\begin{tabular}{|c|c|c|c|c|c|c|c|c|c|}
\hline Disease state & Drug & $\mathrm{CO}_{p}$ & $E_{s}$ & $E_{T}$ & $\mathrm{Cl}_{\mathrm{s}}$ & $C l_{D}$ & $V_{P}$ & $V_{T}$ & Ref \\
\hline ESRD* & Aminoglycosides & $\leftrightarrow$ & $\downarrow$ & ? & $\downarrow$ & ? & 1 & 1 & $\begin{array}{l}{[61]} \\
{[62]} \\
{[63]}\end{array}$ \\
\hline Obesity & Thiopental & 1 & $\uparrow$ & $\leftrightarrow$ & 1 & 1 & 1 & 1 & $\begin{array}{l}{[6]} \\
{[64]} \\
{[65]}\end{array}$ \\
\hline $\mathrm{CHF}^{* *}$ & Lidocaine & $\downarrow$ & $\leftrightarrow$ & $\leftrightarrow$ & $\downarrow$ & $\downarrow$ & $\downarrow$ & $\leftrightarrow$ & [66] \\
\hline $\begin{array}{l}\text { Hypermetabolic } \\
\text { syndrome }\end{array}$ & Aminoglycosides & $\uparrow$ & $\leftrightarrow$ & $?$ & $\uparrow$ & $\uparrow$ & $\uparrow$ & $\uparrow$ & [67] \\
\hline Aging & Thiopental & $\downarrow$ & $\leftrightarrow$ & $\leftrightarrow$ & $\downarrow$ & $\downarrow$ & $\uparrow$ & $\uparrow$ & [65] \\
\hline $\begin{array}{l}\text { ESRD }{ }^{*} \text { End-Stage } \\
\text { CHF** Congestive }^{*} \text { Con }\end{array}$ & $\begin{array}{l}\text { nal Disease } \\
\text { Ieart Failure }\end{array}$ & & & & & & & & \\
\hline
\end{tabular}

Table 2. Disease states with possible influence on the parameters of the recirculatory model for different drugs.

Recirculatory models allow pathophysiological changes related to liver disease to be assessed. The reduction in the area under the curve of the transport function of ICG after the first recirculation reflects the increase in cardiac output characteristic of these patients. After successive recirculations, the change in slope of the ICG profile can reflect changes in the elimination clearance [68].

Another clinical application of recirculatory models is the optimization of pharmacokinetic/ pharmacodynamic (PK/PD) models for the characterization of the rapid response of rocuronium in humans using a recirculatory model coupled to an effect compartment [5]. When compared with a conventional model, the authors found a significant difference between the $\mathrm{k}_{\mathrm{e} 0}$ and $\mathrm{CE}_{50}$ calculated with either model. They also demonstrated a significant correlation between $\mathrm{CO}$ and $\mathrm{k}_{\mathrm{e} 0}$ for both types of models, which was much stronger with the recirculatory 
one. These findings have certain clinical implications since it can be expected that patients with a lower $\mathrm{CO}$ will require a longer time for the effect to be reached [5]. Recently, a population analysis used for fitting the data of rocuronium in patients to a circulatory model has provided insight into the role of vascular mixing and interstitial diffusion. The results suggest that rocuronium is distributed in the interstitial space by passive diffusion where it undergoes diffusion-limited distribution. The body distribution depends on cardiac output and on the apparent permeability surface area product $[0.6 \mathrm{l} / \mathrm{min}$ ). It has also been concluded that the heterogeneity of the blood transit time through the systemic circulation decreases and cardiopulmonary volume increases, respectively, with cardiac output [69].

The observed effect of $\beta$-adrenergic blockers such as propranolol on the disposition of markers such as ICG and antipyrine using a recirculatory model demonstrates that propranolol decreases cardiac output at the expense of the non-splanchnic intravascular circuit. This phenomenon may explain the need for decreased intravenous anesthetic doses in the presence of $\beta$-adrenergic blockade with important clinical implications [18, 70].

\section{Conclusion}

Recirculatory models are a type of physiologically based pharmacokinetic model (PBPK) that offer an alternative to the classic compartmental models and other models in the pharmacokinetic analysis of clinical data.

Although given its complexity the clinical use of these models is limited, they have important advantages in that they are able to characterize the first passage of the drug in the systemic circulation and evaluate changes in cardiac output or regional blood flow and its impact on distribution and elimination clearances induced by disease states. These models are also able to assess changes in the pharmacokinetics associated with concomitant use of other drugs and can be used to perform pharmacokinetic/pharmacodynamic studies (PK/PD). Anesthetics constitute one of the pharmacological groups where this type of model has great clinical interest.

\section{Author details}

José M. Lanao ${ }^{1^{*}}$ and Clara Isabel Colino ${ }^{1}$

*Address all correspondence to: jmlanao@usal.es

1 Department of Pharmacy and Pharmaceutical Technology, Faculty of Pharmacy, University of Salamanca, Spain

Campus Miguel de Unamuno. Facultad de Farmacia, Salamanca, Spain 


\section{References}

[1] Zierler K. Indicator dilution methods for measuring blood flow, volume, and other properties of biological systems: a brief history and memoir. Ann Biomed Eng 2000 Aug;28(8):836-848.

[2] Homer LD, Small A. A unified theory for estimation of cardiac output, volumes of distribution and renal clearance from indicator dilution curves. J Theor Biol 1977 Feb 7;64(3):535-550.

[3] Weiss M, Förster W. Pharmacokinetic model based on circulatory transport. Eur J Clin Pharmacol 1979;16:287-223.

[4] Avram MJ, Krejcie TC, Niemann CU, Enders-Klein C, Shanks CA, Henthorn TK. Isoflurane alters the recirculatory pharmacokinetics of physiologic markers. Anesthesiology 2000 Jun;92(6):1757-1768.

[5] Kuipers JA, Boer F, Olofsen E, Bovill JG, Burm AG. Recirculatory pharmacokinetics and pharmacodynamics of rocuronium in patients: the influence of cardiac output. Anesthesiology 2001 Jan;94(1):47-55.

[6] Wada DR, Bjorkman S, Ebling WF, Harashima H, Harapat SR, Stanski DR. Computer simulation of the effects of alterations in blood flows and body composition on thiopental pharmacokinetics in humans. Anesthesiology 1997 Oct;87(4):884-899.

[7] Valentinuzzi ME, Geddes LA, Baker LE. A simple mathematical derivation of the Stewart-Hamilton formula for the determination of cardiac output. Med Biol Eng 1969 May;7(3):277-282.

[8] Kinsman JM, Moore LJ, Hamilton WF. Studies on the circulation. I. Injection method: physical and mathematical considerations. Am J Physiol 1929;89:322-330.

[9] Fegler G. Measurement of cardiac output in anaesthetized animals by a thermodilution method. Q J Exp Physiol Cogn Med Sci 1954;39(3):153-164.

[10] Vaughan DP, Hope I. Applications of a recirculatory stochastic pharmacokinetic model: limitations of compartmental models. J Pharmacokinet Biopharm 1979 Apr; 7(2):207-225.

[11] Bassingthwaighte JB, Ackerman FH. Mathematical linearity of circulatory transport. J Appl Physiol 1967 May;22(5):879-888.

[12] van Rossum JM, de Bie JE, van Lingen G, Teeuwen HW. Pharmacokinetics from a dynamical systems point of view. J Pharmacokinet Biopharm 1989 Jun;17(3):365-392; discussion 393-400.

[13] Upton RN, Runciman WB, Mather LE. Regional pharmacokinetics. III. Modelling methods. Biopharm Drug Dispos 1991 Jan-Feb;12(1):1-15. 
[14] Weiss M, Krejcie TC, Avram MJ. Transit time dispersion in pulmonary and systemic circulation: effects of cardiac output and solute diffusivity. Am J Physiol Heart Circ Physiol 2006 Aug;291(2):H861-870.

[15] Weiss M. Cardiac output and systemic transit time dispersion as determinants of circulatory mixing time: a simulation study. J Appl Physiol (1985) 2009 Aug;107(2):445449 .

[16] Yano Y, Yamaoka K, Aoyama Y, Tanaka H. Two-compartment dispersion model for analysis of organ perfusion system of drugs by fast inverse Laplace transform (FILT). J Pharmacokinet Biopharm 1989 Apr;17(2):179-202.

[17] Knopp TJ, Dobbs WA, Greenleaf JF, Bassingthwaighte JB. Transcoronary intravascular transport functions obtained via a stable deconvolution technique. Ann Biomed Eng 1976 Mar;4(1):44-59.

[18] Niemann CU, Henthorn TK, Krejcie TC, Shanks CA, Enders-Klein C, Avram MJ. Indocyanine green kinetics characterize blood volume and flow distribution and their alteration by propranolol. Clin Pharmacol Ther 2000 Apr;67(4):342-350.

[19] Schroder T, Rosler U, Hoeft A, Scholz M, Hering JP, Hellige G. Calculation of body transport function. Phys Med Biol 1992 Nov;37(11):2059-2069.

[20] Weiss M, Hubner GH, Hubner IG, Teichmann W. Effects of cardiac output on disposition kinetics of sorbitol: recirculatory modelling. Br J Clin Pharmacol 1996 Apr; 41(4):261-268.

[21] Karalis V, Dokoumetzidis A, Macheras P. A physiologically based approach for the estimation of recirculatory parameters. J Pharmacol Exp Ther 2004 Jan;308(1):198205.

[22] Weiss M. Pharmacokinetics in organs and the intact body: model validation and reduction. Eur J Pharm Sci 1998 Jan;7(2):119-127.

[23] Weiss M, Krejcie TC, Avram MJ. A minimal physiological model of thiopental distribution kinetics based on a multiple indicator approach. Drug Metab Dispos 2007 Sep; 35(9):1525-1532.

[24] Weiss M, Krejcie TC, Avram MJ. Circulatory transport and capillary-tissue exchange as determinants of the distribution kinetics of inulin and antipyrine in dog. J Pharm Sci 2007 Apr;96(4):913-926.

[25] Dokoumetzidis A, Macheras P. A model for transport and dispersion in the circulatory system based on the vascular fractal tree. Ann Biomed Eng 2003 Mar;31(3):284293.

[26] Oliver RE, Jones AF, Rowland M. A whole-body physiologically based pharmacokinetic model incorporating dispersion concepts: short and long time characteristics. J Pharmacokinet Pharmacodyn 2001 Feb;28(1):27-55. 
[27] Avram MJ, Henthorn TK, Spyker DA, Krejcie TC, Lloyd PM, Cassella JV, et al. Recirculatory pharmacokinetic model of the uptake, distribution, and bioavailability of prochlorperazine administered as a thermally generated aerosol in a single breath to dogs. Drug Metab Dispos 2007 Feb;35(2):262-267.

[28] Krejcie TC, Henthorn TK, Shanks CA, Avram MJ. A recirculatory pharmacokinetic model describing the circulatory mixing, tissue distribution and elimination of antipyrine in dogs. J Pharmacol Exp Ther 1994 May;269(2):609-616.

[29] Krejcie TC, Henthorn TK, Niemann CU, Klein C, Gupta DK, Gentry WB, et al. Recirculatory pharmacokinetic models of markers of blood, extracellular fluid and total body water administered concomitantly. J Pharmacol Exp Ther 1996 Sep;278(3):10501057.

[30] Krejcie TC, Avram MJ, Gentry WB, Niemann CU, Janowski MP, Henthorn TK. A recirculatory model of the pulmonary uptake and pharmacokinetics of lidocaine based on analysis of arterial and mixed venous data from dogs. J Pharmacokinet Biopharm 1997 Apr;25(2):169-190.

[31] Upton RN. The two-compartment recirculatory pharmacokinetic model - an introduction to recirculatory pharmacokinetic concepts. Br J Anaesth 2004 Apr;92(4):475484.

[32] Upton RN, Ludbrook GL, Grant C, Martinez AM. Cardiac output is a determinant of the initial concentrations of propofol after short-infusion administration. Anesth Analg 1999 Sep;89(3):545-552.

[33] Upton RN, Ludbrook GL. Pharmacokinetic-pharmacodynamic modelling of the cardiovascular effects of drugs - method development and application to magnesium in sheep. BMC Pharmacol 2005 Mar 10;5:5.

[34] Wiltshire HR, Kilpatrick GJ, Tilbrook GS, Borkett KM. A placebo- and midazolamcontrolled phase I single ascending-dose study evaluating the safety, pharmacokinetics, and pharmacodynamics of remimazolam (CNS 7056): Part II. Population pharmacokinetic and pharmacodynamic modeling and simulation. Anesth Analg 2012 Aug;115(2):284-296.

[35] Upton RN, Ludbrook GL. A physiological model of induction of anaesthesia with propofol in sheep. 1. Structure and estimation of variables. Br J Anaesth 1997 Oct; 79(4):497-504.

[36] Ludbrook GL, Upton RN. A physiological model of induction of anaesthesia with propofol in sheep. 2. Model analysis and implications for dose requirements. Br J Anaesth 1997 Oct;79(4):505-513.

[37] Minto CF, Schnider TW. Contributions of PK/PD modeling to intravenous anesthesia. Clin Pharmacol Ther 2008 Jul;84(1):27-38.

[38] Masui K, Upton RN, Doufas AG, Coetzee JF, Kazama T, Mortier EP, et al. The performance of compartmental and physiologically based recirculatory pharmacokinetic 
models for propofol: a comparison using bolus, continuous, and target-controlled infusion data. Anesth Analg 2010 Aug;111(2):368-379.

[39] Avram MJ, Krejcie TC, Niemann CU, Klein C, Gentry WB, Shanks CA, et al. The effect of halothane on the recirculatory pharmacokinetics of physiologic markers. Anesthesiology 1997 Dec;87(6):1381-1393.

[40] Krejcie TC, Henthorn TK, Gentry WB, Niemann CU, Enders-Klein C, Shanks CA, et al. Modifications of blood volume alter the disposition of markers of blood volume, extracellular fluid, and total body water. J Pharmacol Exp Ther 1999 Dec;291(3):13081316.

[41] Avram MJ, Krejcie TC, Henthorn TK. The concordance of early antipyrine and thiopental distribution kinetics. J Pharmacol Exp Ther 2002 Aug;302(2):594-600.

[42] Avram MJ, Krejcie TC. Using front-end kinetics to optimize target-controlled drug infusions. Anesthesiology 2003 Nov;99(5):1078-1086.

[43] MicroMath Scientific Software. Salt lake City. SCIENTIST 2.0. 1995.

[44] Yano Y, Yamaoka K, Tanaka H. A nonlinear least squares program, MULTI(FILT), based on fast inverse Laplace transform for microcomputers. Chem Pharm Bull (Tokyo) 1989 Apr;37(4):1035-1038.

[45] MAPLESOFT. Technical computer software for engineers. 1988.

[46] Schwinn DA, Shafer SL. Basic Principles of Pharmacology Related to Anesthesia. In: Miller RD, editor. Anesthesiology. 5th edition. New York: Churchill Livingstone; 1999. pp. 15-47.

[47] Krejcie TC, Avram MJ. Recirculatory pharmacokinetic modeling: what goes around, comes around. Anesth Analg 2012 Aug;115(2):223-226.

[48] Upton RN, Grant C, Martinez AM, Ludbrook GL. Recirculatory model of fentanyl disposition with the brain as the target organ. Br J Anaesth 2004 Nov;93(5):687-697.

[49] Lenz K, Gegenhuber A, Firlinger F, Lohr G, Piringer P. Pilot study of levosimendan: effect on liver blood flow and liver function in acute decompensated heart failure. Med Klin Intensivmed Notfmed 2014 May;109(4):267-270.

[50] Nakamura T, Nakamura S, Kaneko T. Flow of portal and hepatic blood in normal and cirrhotic liver. Nature 1961 May 27;190:812-813.

[51] Woosley RL. Pharmacokinetics and pharmacodynamics of antiarrhythmic agents in patients with congestive heart failure. Am Heart J 1987 Nov;114(5):1280-1291.

[52] Rodighiero V. Effects of liver disease on pharmacokinetics. An update. Clin Pharmacokinet 1999 Nov;37(5):399-431.

[53] Benowitz NL, Meister W. Pharmacokinetics in patients with cardiac failure. Clin Pharmacokinet 1976 Nov-Dec;1(6):389-405. 
[54] Piafsky KM. Disease-induced changes in the plasma binding of basic drugs. Clin Pharmacokinet 1980 May-Jun;5(3):246-262.

[55] Brower KLR, Dukes GE, Powell JR. Influence of Liver Function on Drug Disposition. In: Evans WE, Schentag JJ, Jusko WJ, editors. Applied Pharmacokinetics. Principles of Therapeutic Drug Monitoring. Vancouver: Applied Therapeutics Inc; 1992. pp. 6-16-59.

[56] MacKichan JJ. Influence of Protein Binding and Use of Unbound (Free) Drug Concentrations. In: Evans WE, Schentag JJ, Jusko WJ, editors. Applied Pharmacokinetics. Principles of Therapeutic Drug Monitoring. Vancouver: Applied Therapeutics Inc; 1992. pp. 5-1-5-48.

[57] Gawlinski A. Measuring cardiac output: intermittent bolus thermodilution method. Crit Care Nurse 2000 Apr;20(2):118-120, 122-124.

[58] Henthorn TK, Krejcie TC, Avram MJ. The relationship between alfentanil distribution kinetics and cardiac output. Clin Pharmacol Ther 1992 Aug;52(2):190-196.

[59] Andersen V, Sonne J, Larsen S. Antipyrine, oxazepam, and indocyanine green clearance in patients with chronic pancreatitis and healthy subjects. Scand J Gastroenterol 1999 Aug;34(8):813-817.

[60] Zaske DE. Aminoglycosides. In: Evans WE, Schentag JJ, Jusko WJ, editors. Applied Pharmacokinetics. Principles of Therapeutic Drug Monitoring. Vancouver: Applied Therapeutics Inc.; 1992. pp. 14-1-14-47.

[61] Lanao JM, Dominguez-Gil A, Tabernero JM, De Castro S. Pharmacokinetics of Amikacin (BB-K8) in patients with normal or impaired renal function. Int J Clin Pharmacol Biopharm 1979 Apr;17(4):171-175.

[62] Gambertoglio JG. Effects of Renal Disease; Altered Pharmacokinetics. In: Benet LZ, Massoud N, Gambertoglio JG, editors. Pharmacokinetic Basis for Drug Treatment. New York: Raven Press; 1984. pp. 149-172.

[63] Matzke GR, Keane WF. Use of Antibiotics in Renal Failure. In: Petersen PK, Verhoef J, editors. The Antimicrobial Agents Annual. Amsterdam: Elsevier; 1986. pp. 472-488.

[64] Jung D, Mayersohn M, Perrier D, Calkins J, Saunders R. Thiopental disposition in lean and obese patients undergoing surgery. Anesthesiology 1982 Apr;56(4):269-274.

[65] Avram MJ, Krejcie TC, Henthorn TK. The relationship of age to the pharmacokinetics of early drug distribution: the concurrent disposition of thiopental and indocyanine green. Anesthesiology 1990 Mar;72(3):403-411.

[66] Thomson PD, Melmon KL, Richardson JA, Cohn K, Steinbrunn W, Cudihee R, et al. Lidocaine pharmacokinetics in advanced heart failure, liver disease, and renal failure in humans. Ann Intern Med 1973 Apr;78(4):499-508. 
[67] Pennington JE, Dale DC, Reynolds HY, MacLowry JD. Gentamicin sulfate pharmacokinetics: lower levels of gentamicin in blood during fever. J Infect Dis 1975 Sep; 132(3):270-275.

[68] Niemann CU, Yost CS, Mandell S, Henthorn TK. Evaluation of the splanchnic circulation with indocyanine green pharmacokinetics in liver transplant patients. Liver Transpl 2002 May;8(5):476-481.

[69] Weiss M, Reekers M, Vuyk J, Boer F. Circulatory model of vascular and interstitial distribution kinetics of rocuronium: a population analysis in patients. J Pharmacokinet Pharmacodyn 2011 Apr;38(2):165-178.

[70] Avram MJ, Krejcie TC, Henthorn TK, Niemann CU. Beta-adrenergic blockade affects initial drug distribution due to decreased cardiac output and altered blood flow distribution. J Pharmacol Exp Ther 2004 Nov;311(2):617-624. 


\section{Edited by Tarek A Ahmed}

This book considers the basic principles of biopharmaceutics and pharmacokinetics. It also illustrates clinical pharmacokinetic applications, such as recirculatory models, common antimalarial drugs, and clinical pharmacokinetic principles in critically

ill patients, which are essential for medical professionals. Undergraduate and postgraduate students can make use of the information presented. The contents of the book represent the authors points of view as well as clinical findings and basic concepts of pharmacokinetics and biopharmaceutics that are covered in textbooks. 